Florida International University FIU Digital Commons

9-22-2017

\title{
The Detection of an Invasive Pathogen through Chemical and Biological Means for the Protection of Commercial Crops
}

Alison G. Simon

asimo046@fiu.edu

DOI: $10.25148 /$ etd.FIDC004011

Follow this and additional works at: https://digitalcommons.fiu.edu/etd

Part of the Chemistry Commons

\section{Recommended Citation}

Simon, Alison G., "The Detection of an Invasive Pathogen through Chemical and Biological Means for the Protection of Commercial Crops" (2017). FIU Electronic Theses and Dissertations. 3558.

https://digitalcommons.fiu.edu/etd/3558 


\section{FLORIDA INTERNATIONAL UNIVERSITY}

Miami, Florida

THE DETECTION OF AN INVASIVE PATHOGEN THROUGH CHEMICAL AND BIOLOGICAL MEANS FOR THE PROTECTION OF COMMERCIAL CROPS

A dissertation submitted in partial fulfillment of

the requirements for the degree of
DOCTOR OF PHILOSOPHY
in
CHEMISTRY
by

Alison G. Simon 
To: Dean Michael R. Heithaus

College of Arts, Science, and Education

This dissertation, written by Alison G. Simon, and entitled The Detection of an Invasive Pathogen through Chemical and Biological Means for the Protection of Commercial Crops, having been approved in respect to style and intellectual content, is referred to you for judgment.

We have read this dissertation and recommend that it be approved.

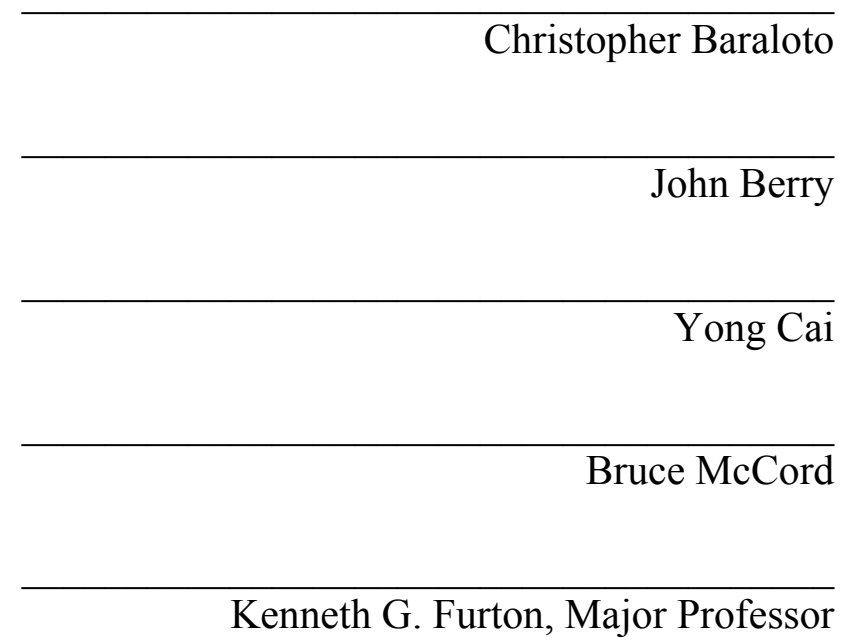

Date of Defense: September 22, 2017

The dissertation of Alison G. Simon is approved.

Dean Michael R. Heithaus

College of Arts, Sciences, and Education

Andres G. Gil

Vice President for Research and Economic Development

and Dean of the University Graduate School

Florida International University, 2017 
(C) Copyright 2017 by Alison G. Simon

All rights reserved. 


\section{DEDICATION}

This dissertation is dedicated to my late mentor who first introduced me to the world of canine science, Rex Stockham, and to my family and friends, without whose support (and wine) it could not have been completed. 


\section{ACKNOWLEDGMENTS}

I would like to acknowledge all the people and facilities that were necessary to my research. This work was supported by a State of Florida Specialty Crop Block Grant (\#020731) to DeEtta K. Mills and Kenneth G. Furton; the J. Edgar Hoover Foundation, and the Society of Former Special Agents of the FBI Foundation. Fungal cultures were provided by the Tropical Research and Education Center of the University of Florida (Homestead, FL). Dr. Scott Zona and the Wertheim Conservatory; the International Forensic Research Institute's Trace Evidence Analytical Facility; and the Florida Center for Analytical Electron Microscopy each provided necessary resources and instrumentation. Selected DNA analysis was done by Christina Burns and Julian Mendel. Many samples of avocado tree were supplied by farmers in South Florida. Canine trials could not have occurred without the participation of Innovative Detection Concepts (Redlands, FL). I want to thank particularly several Furtonites, especially Jeremy Guedes, Alex Echevarria, and Valeria Torres, for assistance with laboratory work. Others who were essential to my research are Meghan Roig (who actually knows biology), Laura Conklin (who helped fight the banana spiders), and Ben Claassen (whose clever map of the fungus is included herein). Thank you!

On my first day in lab, I set to work making standards. And I did it wrong. Iris and Michelle stood by, discussing how long they'd let me go on before correcting me. Since then, I've learned an incredible amount of science from a diverse group of people, but just as important are the people who kept me (kind of) sane throughout this entire process. From wine nights to coffee breaks, some friends let me escape for a little bit from the stresses that are a $\mathrm{PhD}$. Other friends were travel buddies, helping me to escape on a larger scale. Remember 
everyone, when in doubt...Denzel. Finally, I want to thank my family. I am lucky enough to have four amazing siblings that support me and parents that would do anything for me. At a moment's notice Dad would drive thirteen hours if I so much as needed a shoulder to lean on. Mom especially was my on-call supporter. 
ABSTRACT OF THE DISSERTATION

THE DETECTION OF AN INVASIVE PATHOGEN THROUGH CHEMICAL AND

BIOLOGICAL MEANS FOR THE PROTECTION OF COMMERCIAL CROPS

by

Alison G. Simon

Florida International University, 2017

Miami, Florida

Professor Kenneth G. Furton, Major Professor

Standoff detection of targets using volatiles is essential when considering substances that are hazardous or dangerous, or for which the presence or location is unknown. For many invasive biological threats, their presence is often not realized until they have begun visibly affecting and spreading through crops or forests. The fungus Raffaelea lauricola is a biothreat vectored by the invasive beetle Xyleborus glabratus, or redbay ambrosia beetle (RAB), whose presence in avocado groves is currently detectable by visual inspection. Once visually identified, the affected trees must be removed and destroyed to protect those remaining trees. However, if the fungus is identified via standoff volatile detection, there is anecdotal evidence that it can be treated with propiconazole and saved from progression to the fatal laurel wilt disease. As a result of the rapid spread of $R$. lauricola and the quick death of trees, early detection through standoff methods is essential. The only current method of pre-symptomatic identification is canine detection. Canines are sensitive and selective biological detectors that can trace odors to their source, despite the presence of a variety of background odors. The present research evaluated the volatile organic compounds (VOCs) of the laurel wilt disease and $R$. lauricola using headspace solid phase microextraction-gas chromatography-mass 
spectrometry (HS-SPME-GC-MS). Additionally, a new method for odor collection and presentation to trained detection canines was developed. Knowledge of the disease and standoff volatile detection capabilities are improved using this information. 


\section{TABLE OF CONTENTS}

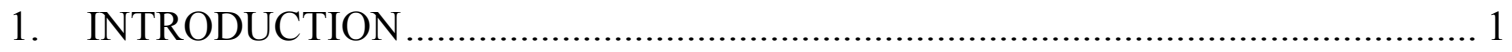

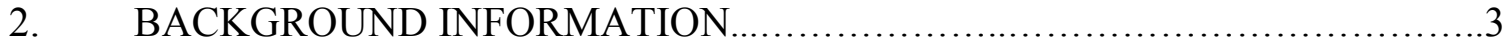

2.1. Volatile Organic Compounds Released in Nature .......................................................... 3

2.2. Global Biotic Homogenization of Ambrosia Beetles ..................................................... 9

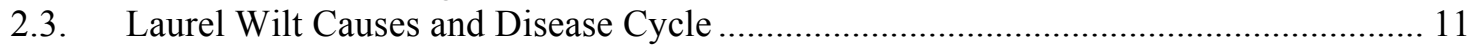

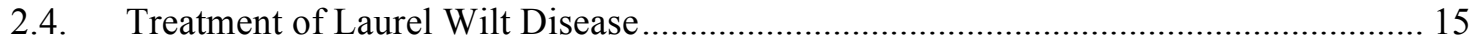

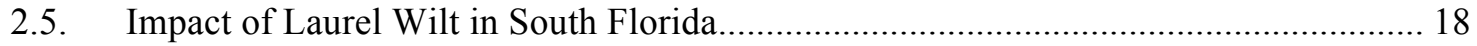

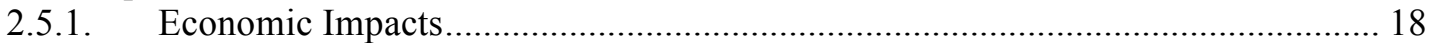

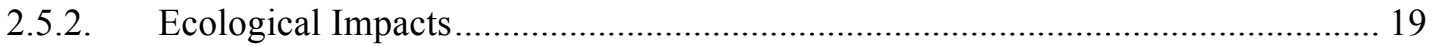

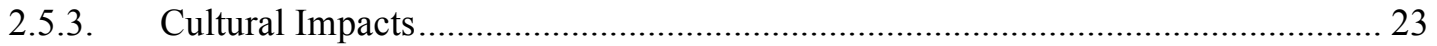

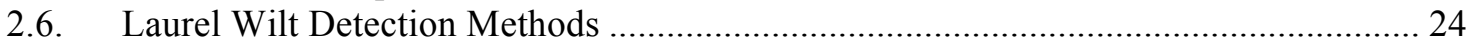

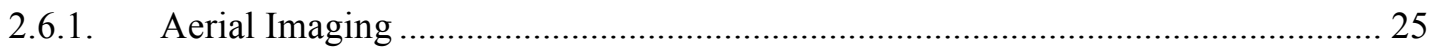

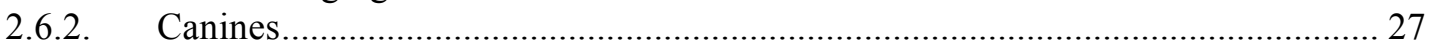

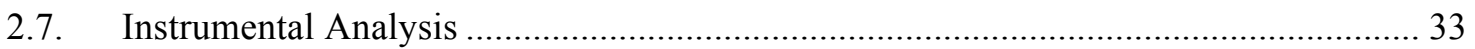

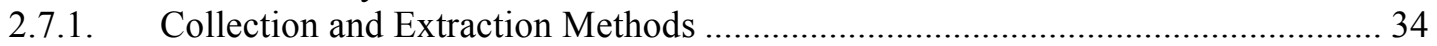

2.7.2. Gas Chromatography-Mass Spectrometry ........................................................ 38

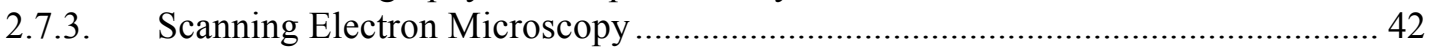

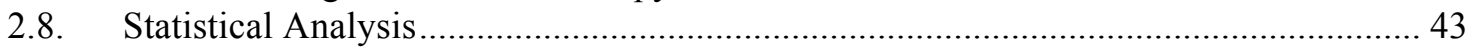

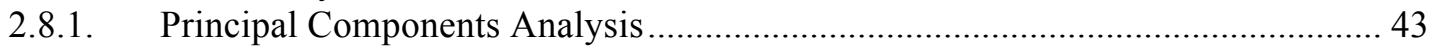

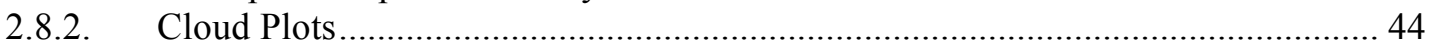

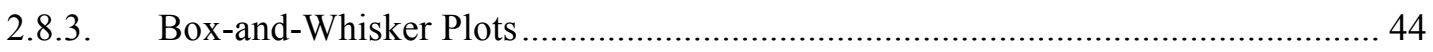

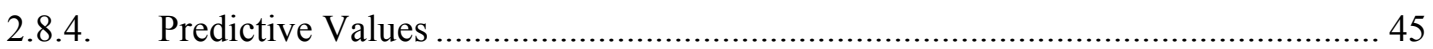

3. SIGNIFICANCE OF STUDY AND RESEARCH OBJECTIVES........................... 46

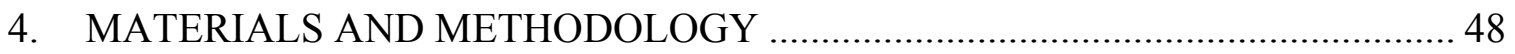

4.1. Task 1: Optimization of a Method for the Detection of the Volatile Organic

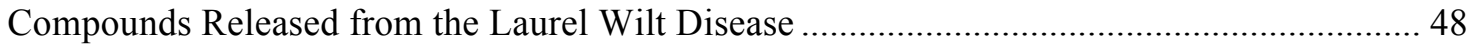

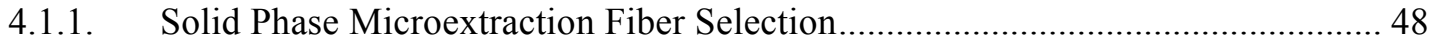

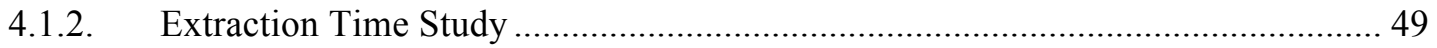

4.1.3. Gas Chromatography-Mass Spectrometry Optimization..................................... 50

4.2. Task 2: Analytical Studies of the Laurel Wilt Disease ................................................... 50

4.2.1. Examining Volatile Organic Compounds of Raffaelea lauricola Over Time ....... 50

4.2.2. Establishing Volatile Organic Compounds of Infected Avocado Trees and

Comparing Volatile Organic Compounds from Infected Avocado Trees to Those of

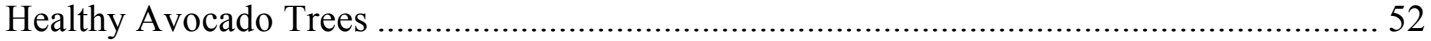

4.3. Task 3: Assessment of a Controlled Odor Mimic Permeation System for the

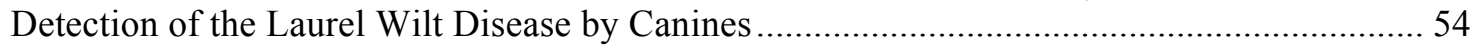

4.3.1. Development of the Column Vent Method for Active Odor Identification............ 54

4.3.2. Characterization of the Canine Training Aid Used in the Field ............................ 59

4.4. Task 4: Evaluation of the Volatile Organic Compounds of the Laurel Wilt Disease in

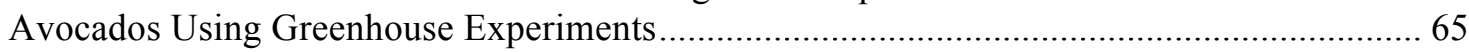

4.4.1. Examining the Volatile Organic Compounds of Avocado Trees Inoculated with

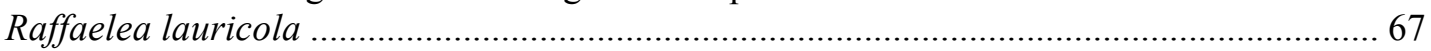

4.4.2. Examining the Volatile Organic Compounds of Treated Infected Avocado Trees 67

5. RESULTS AND DISCUSSION. 
5.1. Task 1: Optimization of a Method for the Detection of the Volatile Organic

Compounds Released from the Laurel Wilt Disease

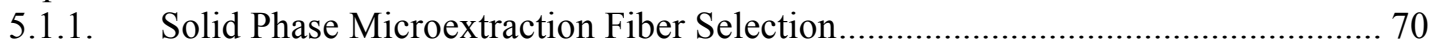

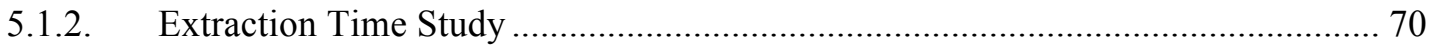

5.1.3. Gas Chromatography-Mass Spectrometry Optimization.................................... 72

5.2. $\quad$ Task 2: Analytical Studies of the Laurel Wilt Disease ............................................... 73

5.2.1. Examining the Volatile Organic Compounds of Raffaelea lauricola Over Time 74

5.2.2. Establishing Volatile Organic Compounds of Infected Avocado Trees................. 82

5.2.3. Comparing Volatile Organic Compounds from Infected Avocado Trees to

Those of Healthy Avocado Trees

5.3. Task 3: Assessment of a Controlled Odor Mimic Permeation System for the

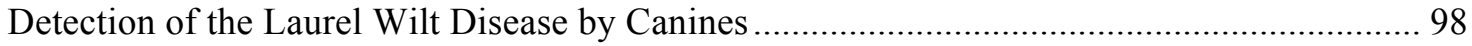

5.3.1. Development of the Column Vent Method for Active Odor Identification........... 99

5.3.2. Characterization of the Canine Training Aid Used in the Field .......................... 103

5.4. Task 4: Evaluation of Volatile Organic Compounds of the Laurel Wilt Disease in

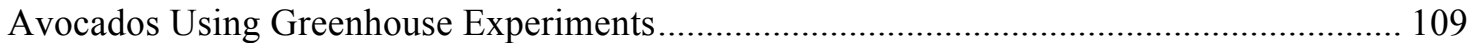

5.4.1. Examining the Volatile Organic Compounds of Avocado Trees Inoculated with

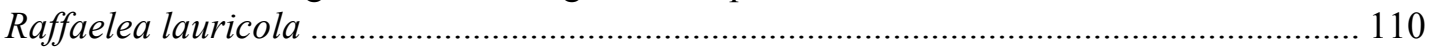

5.4.2. Examining the Volatile Organic Compounds of Treated Inoculated Avocado

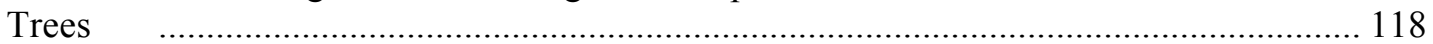

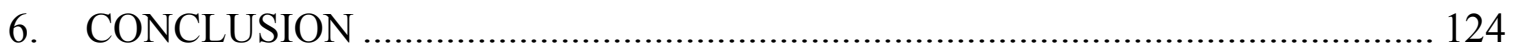

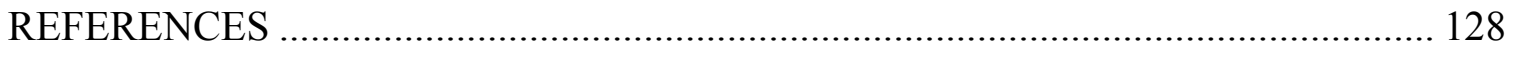

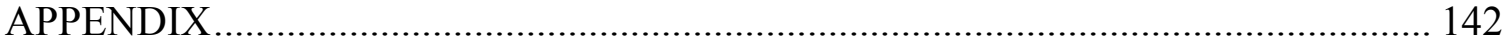

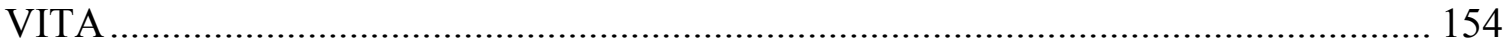




\section{LIST OF TABLES}

TABLE

PAGE

Table 1: The VOCs previously identified from trunk samples of avocado trees. 6

Table 2: Hosts of $R$. lauricola by the year and location of identification 21

Table 3: Examples of commercially available SPME fibers 35

Table 4: Possible canine decision or search outcomes 46

Table 5: The temperature ramp used for the GC-MS analyses (1) 54

Table 6: The parameters for the Varian 3800 Gas Chromatograph/Saturn 2000 Ion Trap Mass Spectrometer. 54

Table 7: The parameters for the Bruker Scion 346-Gas Chromatograph ....................... 55

Table 8: Division of fractions by minute for column vent procedure............................. 56

Table 9: Percent loss between direct liquid injection and SPME ................................ 59

Table 10: Relevant information for naphthalene- $\mathrm{D}_{8}$ and target compounds .................. 61

Table 11: The parameters for the Agilent Technologies HP 6890 Series Gas

Chromatography System/HP 5973 Mass Selective Detector 63

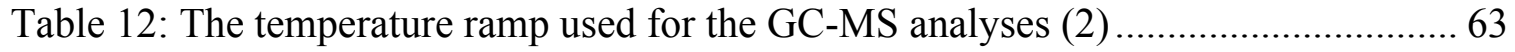

Table 13: Compounds present in at least $80 \%$ of Lula variety avocado tree samples...... 83

Table 14: Compounds present in avocado tree injured by lightning ............................ 93

Table 15: Compounds present in healthy and infected wild swampbay trees ................. 96

Table 16: Canine trial results presented as alert rate (\%) and no alert rate (\%) ............ 101

Table 17: Location and relevant weather data for samples collected by the authors ..... 142 
Table 18: Laboratory parameters from Task 4.4.1 (Continued next page).................... 143

Table 19: Laboratory parameters from Task 4.4.2 (Continued next two pages) ............ 145 


\section{LIST OF FIGURES}

FIGURE

PAGE

Figure 1: Products of the TCA cycle and MVA pathway in plants, stemming from glycolysis. Adapted from Schnürer et al. (1999) ${ }^{14}$. 4

Figure 2: The MVA pathway in plants showing the creation of monoterpenes and sesquiterpenes.

Figure 3: Map of the spread of $R$. lauricola since entry into the United States (as of April 2017). Black stripes indicate avocado growing regions. *Affected counties in Virginia not indicated.

Figure 4: The disease cycle of laurel wilt. 14

Figure 5: Diagram showing VIS-NIR wavelengths used to identify injured trees. 25

Figure 6: Drone fitted with camera used to capture aerial images of avocado groves. .... 27

Figure 7: Example of COMPS. 33

Figure 8: SPME fiber diagram. 36

Figure 9: Basic SPME-ESIS diagram. 37

Figure 10: Vapor pressure bag with connected handheld vacuum pump. 38

Figure 11: Basic GC-MS diagram. 40

Figure 12: Example of a box-and-whisker plot.

Figure 13: Schematic showing sample selection in avocado groves. There is a two-row barrier of non-symptomatic trees between infected and uninfected samples. 52

Figure 14: Fraction being collected on cotton gauze in $10 \mathrm{~mL}$ glass vial from $0.53 \mathrm{~mm}$ ID solgel-wax column vented to the atmosphere 55

Figure 15: Design of canine experiments over three days. (a) Took place in a mango grove with $6 \mathrm{mph}$ (10kph) SE winds. (b) Took place in a longan grove with $10 \mathrm{mph}$ 
(16kph) NE winds. (c) Took place in a mango grove with $15 \mathrm{mph}(24 \mathrm{kph})$ wind gusts in the SW direction.

Figure 16: Image of COMPS suspended for static testing

Figure 17: SPME sampling method for static testing (COMPS enclosed in a glass vial with a SPME fiber exposed above).

Figure 18: SPME sampling method for dynamic testing (COMPS in an open glass container with SPME fiber exposed above).

Figure 19: Field guide for avocado tree macroinfusion with propiconazole.

Figure 20: Peak areas of compounds recovered from SPME extraction using various fiber chemistries.

Figure 21: Peak areas of compounds recovered from SPME extraction using various extraction times.

Figure 22: Naphthalene-D 8 SPME fiber exposure optimization for ESIS-SPME 72

Figure 23: VOC profiles of $R$. lauricola throughout observation period. No exact specification was possible for terpenes, and their identities as terpenes were based on mass spectral peaks.

Figure 24: PCA produced by XCMS Online showing separation in datasets by day....... 76

Figure 25: Cloud plot produced by XCMS Online showing separation in Days 1-14 (upper TIC) and Days 15-28 (lower TIC) by presenting aligned chromatograms (TIC) plotted based on retention time (minutes) on the $x$-axis and $\mathrm{m} / \mathrm{z}$ on the $\mathrm{y}$-axis. The circles represent detected metabolite features with $\mathrm{p}$-value $\leq 0.01$ and fold change $\geq 1.5$. Green circles indicated toggle upregulated features, while red circles indicate toggle downregulated features. Low p-values are represented by darker colors and larger circle radii indicate larger fold changes

Figure 26: Retention time (minutes) 6.25 through 9 (zoom in from Figure 25) from cloud plot produced by XCMS Online with a box-and-whisker plot showing the toggle downregulation of the feature identified using a mass spectral library as 4,5-dimethyl -1-hexene

Figure 27: Retention time (minutes) 29.5 through 31.75 (zoom in from Figure 25) from cloud plot produced by XCMS Online with a box-and-whisker plot showing the toggle 
downregulation of the feature identified using a mass spectral library as phenylethyl alcohol and the toggle upregulation of the feature identified using a mass spectral library as a terpene.

Figure 28: Various ring closures of farnesene (center molecule) leading to nine identified VOCs in avocado wood samples, where (a) is the eudesmene backbone; (b) is the guaiene backbone; (c) is the germacrene backbone; (d)-(g) are is the cadinene, muurolene, \& calamenene backbone; and (h) is the humulene backbone.

Figure 29: Ring closures of C-1 and C-6 plus C-5 and C-10 leading to the category of sesquiterpenes to include copaene and $\delta$-cadinene.

Figure 30: Ring closures of C-1 and C-6 plus C-5 and C-10 leading to the category of sesquiterpenes to include $\gamma$-muurolene.

Figure 31: Ring closures of C-1 and C-6 plus C-5 and C-10 leading to the category of sesquiterpenes to include calamenene.

Figure 32: Ring closures of $\mathrm{C}-1$ and $\mathrm{C}-10$ leading to the germacrene category of sesquiterpenes.

Figure 33: Ring closures of C-1 and C-11 leading to the humulene category of sesquiterpenes.

Figure 34: Odor profiles of compounds identified in early and late stages of laurel wilt infection in avocado trees. Purple shades indicate compounds present in both odor profiles. Green shades indicate compounds present only in the odor profile of late stage infection. Warm shades (red, orange, and pink) indicate compounds present only in the odor profile of early stage infection.

Figure 35: Ring closures of C-1 and C-10 plus C-2 and C-7 leading to the category of sesquiterpenes to include $\alpha$-selinene.

Figure 36: Ring closures of C-1 and C-10 plus C-2 and C-6 leading to the pseudoguaiene sub-category of sesquiterpenes to include $\alpha$-cubenene.

Figure 37: Ring closures of C-1 and C-10 plus C-2 and C-6 leading to the pseudoguaiene sub-category of sesquiterpenes to include (-)-alloaromadendrene.

Figure 38: Odor profiles of avocado trees struck by lightning, infected with laurel wilt, and uninfected (neither struck by lightning nor infected with laurel wilt). Purple shades indicate compounds present in all three odor profiles. Green shades indicate 
compounds present in just those odor profiles of lightning struck and healthy trees. Blue shades indicate compounds present in only the lightning struck tree odor profile. . 94

Figure 39: PCA of infected avocado tree odors (orange circle), lightning struck avocado tree (blue circle), and healthy avocado tree odors.

Figure 40: PCA of infected avocado tree odors (orange circle), infected swampbay tree odors (purple circle), healthy swampbay tree odors (blue circle), and healthy avocado tree odors. 96

Figure 41: Odor profiles of healthy and infected swampbay trees, and healthy and infected avocado trees. Purple shades indicate compounds present in all four odor profiles. Blue shades indicate compounds present in both healthy and infected swampbay odor profiles, while green shades indicate compounds present in both healthy and infected avocado odor profiles. Warm shades (red, orange, and pink) indicate all other compounds.

Figure 42: Flow chart of the organizational design of the column vent method. Fractions are collected on cotton gauze from GC column vented to the atmosphere. Then, the fractions are confirmed through GC-MS. Finally, the fractions are presented to canines during field trials 100

Figure 43: Chromatograms of fractions A, B, C, D, and W presented to canines. On the chromatograms representing fractions $\mathrm{A}, \mathrm{B}, \mathrm{C}$, and $\mathrm{D}$, the compounds contained in each vial are highlighted. Compounds are labeled in fraction $\mathrm{W}: 1) \alpha$-cubenene, 2) $\delta$-elemene, 3) copaene, 4) (-)-alloaromadendrene, 5) $\gamma$-muurolene, 6) $\alpha$-selinene, 7) $\delta$-cadinene, 8) calamenene, and 9) caryophyllene oxide. 100

Figure 44: Examples of COMPS for laurel wilt detection canines 104

Figure 45: Odor profiles of infected COMPS collected during static sampling by day. 105

Figure 46: Peak area for each compound recovered from static sampling procedures for infected COMPS.

Figure 47: $A / E$ ratios for each compound recovered from static sampling procedures for infected COMPS, plus the total permeation rate.

Figure 48: Odor profiles (given in peak area) of infected COMPS collected during dynamic sampling by day. 
Figure 49: $A / E$ ratios for each compound recovered from dynamic sampling procedures for infected COMPS, plus the total permeation rate. 108

Figure 50: Odor profile of avocado trees inoculated with $R$. lauricola by sample day.. 111

Figure 51: Odor profile of control avocado trees by sample day.

Figure 52: PCA of inoculated and control samples from the greenhouse experiment. The red circle contains inoculated samples. The green circle contains control samples from days $0-21$. The blue circle contains control samples from days 27-51.

Figure 53: Dendrogram showing relations between samples of inoculated and control trees.

Figure 54: Images of the trees on day 0 (left); one inoculated tree after three weeks (middle); and one control tree after three weeks (right).

Figure 55: SEM images of inoculated tree (left) and control tree (right). Green arrows indicate some of the tyloses that can be seen in the image.

Figure 56: Odor profile of avocado trees inoculated with $R$. lauricola and then treated with propiconazole by sample day

Figure 57: Odor profile of avocado trees inoculated with $R$. lauricola and left untreated by sample day

Figure 58: Odor profile of control avocado trees (not inoculated with $R$. lauricola but treated with propiconazole) by sample day.

Figure 59: PCA of inoculated then treated, inoculated then untreated, and control/ treated samples from the greenhouse experiment. The red circle contains inoculated but untreated samples. The green circle contains control/treated samples. The blue circle contains inoculated and treated samples.

Figure 60: SEM images of inoculated and untreated tree (left), inoculated and treated tree (middle), and control but treated tree (right).

Figure 61: Images of the trees (clockwise from upper left) on day 0; one inoculated tree on day 32; one inoculated then treated tree on day 32; and one control tree on day 32 
Figure 62: Relevant weather data collected for trees located in the greenhouse for Task 4 greenhouse experiments of avocado trees inoculated with $R$. lauricola.

Figure 63: Relevant weather data collected for trees located outside for Task 4 greenhouse experiments of avocado trees inoculated with $R$. lauricola

Figure 64: Relevant weather data collected for trees located in the greenhouse for Task 4 greenhouse experiments of avocado trees inoculated with $R$. lauricola and then treated with propiconazole.

Figure 65: Relevant weather data collected for trees located outside for Task 4 greenhouse experiments of avocado trees inoculated with $R$. lauricola and then treated with propiconazole.

Figure 66: Odor profile of avocado trees inoculated with $R$. lauricola by sample day for Section 5.4.1.

Figure 67: Odor profile of control avocado trees by sample day for Section 5.4.1....... 150

Figure 68: Odor profile of avocado trees inoculated with $R$. lauricola and then treated with propiconazole by sample day for Section 5.4.2.

Figure 69: Odor profile of avocado trees inoculated with $R$. lauricola and left untreated by sample day for Section 5.4.2.

Figure 70: Odor profile of control avocado trees (not inoculated with $R$. lauricola but treated with propiconazole) by sample day for Section 5.4.2.

Figure 71: PCA biplot (created from non-normalized data) for greenhouse experiment presented in Section 5.4.1.17_079 is $\beta$-caryophyllene, 14_356 is $\alpha$-cubebene, and $15 \_121$ is copaene.

Figure 72: PCA biplot (created from non-normalized data) for greenhouse experiment presented in Section 5.4.2. 18_0549 is $\beta$-caryophyllene, $16 \_187$ is $\alpha$-cubebene, $18 \_233$ is an unidentified sesquiterpene, 20_007 is $\delta$-selinene, 21_116 is $\delta$-cadinene, and 19_285 is alloaromadendrene. 


\section{LIST OF ABBREVIATIONS}

\begin{tabular}{|c|c|}
\hline Acetyl-CoA & Acetyl coenzyme A \\
\hline CAR/PDMS & Carboxen/polydimethylsiloxane \\
\hline COMPS & Controlled odor mimic permeation system \\
\hline DMAPP & Dimethylallyl diphosphate \\
\hline $\mathrm{DVB} / \mathrm{CAR}$ & Divinylbenzene/Carboxen \\
\hline DVB/CAR/PDMS & Divinylbenzene/Carboxen/polydimethylsiloxane \\
\hline EI & Electron ionization \\
\hline ESIS & Externally sampled internal standard \\
\hline EXR & Excess red \\
\hline FPP & Farnesyl diphosphate \\
\hline GC & Gas chromatography \\
\hline GDVI & Green difference vegetation index \\
\hline GPP & Geranyl diphosphate \\
\hline HS & Headspace \\
\hline IFRI/NFSTC & $\begin{array}{l}\text { International Forensic Research } \\
\text { Institute/National Forensic Science Technology } \\
\text { Center }\end{array}$ \\
\hline IUCN & International Union for Conservation of Nature \\
\hline LDPE & Low density polyethylene \\
\hline MS & Mass spectrometry/mass spectrometer \\
\hline MVA & Mevalonate acid \\
\hline NDVI & Normalized difference vegetation index \\
\hline NIR & Near infrared \\
\hline
\end{tabular}




\begin{tabular}{ll} 
NPCI & Normalized pigment chlorophyll index \\
NPV & Negative predictive value \\
PA & Polyacrylate \\
PCA & Principle components analysis \\
PDA & Potato dextrose agar \\
PDMS & Polydimethylsiloxane \\
PEG & Polyethylene glycol \\
PPV & Positive predictive value \\
RAB & Redbay ambrosia beetle \\
RGRI & Red green ratio index \\
SAR & Systemic acquired resistance \\
SEM & Scanning electron microscopy \\
SPME & Solid phase microextraction \\
SRI & Spectral reflectance index \\
SWGDOG & Scientific Working Group on Dog and \\
TCA & Orthogonal detector Guidelines \\
UHP & Tricarboxylic acid \\
VIS-NIR & Ultra high purity \\
VOC & Visible-near infrared reflectance spectroscopy \\
XCMS & Volatile organic compound \\
& spectrometry \\
\hline
\end{tabular}




\section{INTRODUCTION}

Raffaelea lauricola is a newly described nutritional ambrosia fungus that entered the United States in the early 2000s. The fungus $R$. lauricola is believed to be spread through its symbiotic vector, Xyleborus glabratus, or the redbay ambrosia beetle (RAB), which carries the fungus to farm it for food within host trees. The phytopathogen causes the fatal laurel wilt disease, which is spreading throughout the southeastern United States, most notably in Florida commercial avocado groves and essential ecosystems. Laurel wilt is a vascular disease that causes trees to die by shutting down the flow of water and nutrients in the xylem ${ }^{1}$.

The mechanisms of growth, movement, and life cycle of $R$. lauricola are poorly understood because it is the first ambrosia fungus that is known to spread at such a rapid rate ${ }^{1,2}$, and little is known about the mechanisms of vascular wilt pathogens in general ${ }^{3}$. It is known, however, that fungi, insects, and plants communicate through and defend themselves using volatile organic compounds (VOCs). The present study exploits the communication system by characterizing $R$. lauricola and the laurel disease using VOC analyses of $R$. lauricola, infected and healthy adult avocado trees, and inoculated young avocado trees for detection purposes.

Volatiles are used by canines as a standoff detection method to locate or identify the presence (or absence) of target substances. Trained detection canines are currently one of the few successful methods for early detection of pre-symptomatic diseased avocado trees (Persea americana) in South Florida ${ }^{86}$. There are three trained canine detection teams in the country that can identify the disease before the appearance of visual symptoms. These biological detectors offer rapid onsite identification, are sensitive and selective, and can trace odors to their source, even in the presence of significant background odor. Currently, the canine teams 
utilize controlled odor mimic permeation systems (COMPS) containing infected wood as training aids. The COMPS are a patent pending system of odor containment that allows odor availability to be measured and dissipation rates to be determined. However, successful detection canines require more accessible, safe, and long-lasting training aids to achieve universal and frequent training. To create canine training aids, ideally, these aids would utilize odorants identified through chemical analysis, but identification of odorants and compounds is limited by several factors, including both the availability of chemicals and the need to present chemicals individually and in combination to detection canines. To overcome these challenges, COMPS were first characterized to provide relevant information, such as shelf-life. Then, an analytical method for the separation and identification of VOCs from environmental substances was designed by venting a gas chromatography column to the atmosphere and collecting the fractions. Ten minute fractions of the vented odor profile were collected on cotton gauze in glass vials and presented to the detection canines in a series of field trials. The innovative fractionation and collection method can be used to develop reliable and cost effective canine training aids.

Invasive and novel pests and diseases cause irreparable damage to local agriculture and agricultural communities. Laurel wilt has the potential to eradicate the United States and Mexico avocado industries - causing billions of dollars in losses — especially in Florida, where avocado is the second most important tropical fruit crop. The current research characterized the VOCs of the fungus, the disease, and canine training aids. The knowledge gained by understanding the VOCs associated with these various aspects may then be used to help fight 
the biological advancement of the laurel wilt disease, and help mitigate the impact of its ecological and economic effects.

\section{BACKGROUND INFORMATION}

\subsection{Volatile Organic Compounds Released in Nature}

Volatile organic compounds (VOCs) are carbon-based compounds that enter the gas phase at $0.01 \mathrm{kPa}$. Within plants, these compounds are products or byproducts of biological processes, for example the tricarboxylic acid (TCA) cycle and the mevalonate acid (MVA) pathway (Figure 1). Primary metabolism in such organisms describes the chemical processes that are necessary for life, which includes the TCA cycle. Meanwhile, secondary metabolism describes specialized pathways that aid in survival but are not required to sustain organismal growth. Secondary metabolites are also more important in microbial communications ${ }^{4}$. For example, terpene biosynthesis occurs in the MVA pathway ${ }^{5,6}$, a secondary metabolic pathway stemming from acetate coenzyme A (acetyl-CoA) (Figure 2). In the acetyl-CoA pathway, isopentyl diphosphate and its isomer dimethylallyl diphosphate (DMAPP) form isoprene units that serve as the building blocks for terpenes. Converted by terpene synthases, monoterpenes $\left(\mathrm{C}_{10}\right)$ then go through the intermediate geranyl diphosphate (GPP), and sesquiterpenes $\left(\mathrm{C}_{15}\right)$ continue through the intermediate farnesyl diphosphate (FPP) ${ }^{7}$. The formation of secondary metabolites, such as terpenes, is associated with morphological developments after active growth has ended ${ }^{6,8,9}$. These terpenes display qualitative and quantitative differences among species as well as among varieties and individuals of a single species ${ }^{4,7,10}$. 
Volatiles in nature are used to mediate interactions between organisms such as plants, fungi, insects, and animals ${ }^{2-4,11-13}$. For example, they function in direct plant defenses or as signals in indirect defense responses ${ }^{2}$. Terpenes are the largest and most structurally diverse class of plant secondary metabolites, and are often the mediaries for such plant-plant, plantinsect, or plant-pathogen interactions $\mathrm{s}^{3,13,15,16}$. For instance, a plant damaged by herbivore activity, pathogen inoculation, or even natural stress such as a lightning strike will produce terpenes in response, either attracting other herbivores or those that prey on herbivores, or initiating other biosynthetic pathways or regulatory mechanisms to repair the damage or instigate antibiotic activity. These terpenes also serve as warnings to neighboring plants, and many uninjured plants will produce the same volatiles as their neighboring injured plant to help prime their defenses $3,13,15,16$.

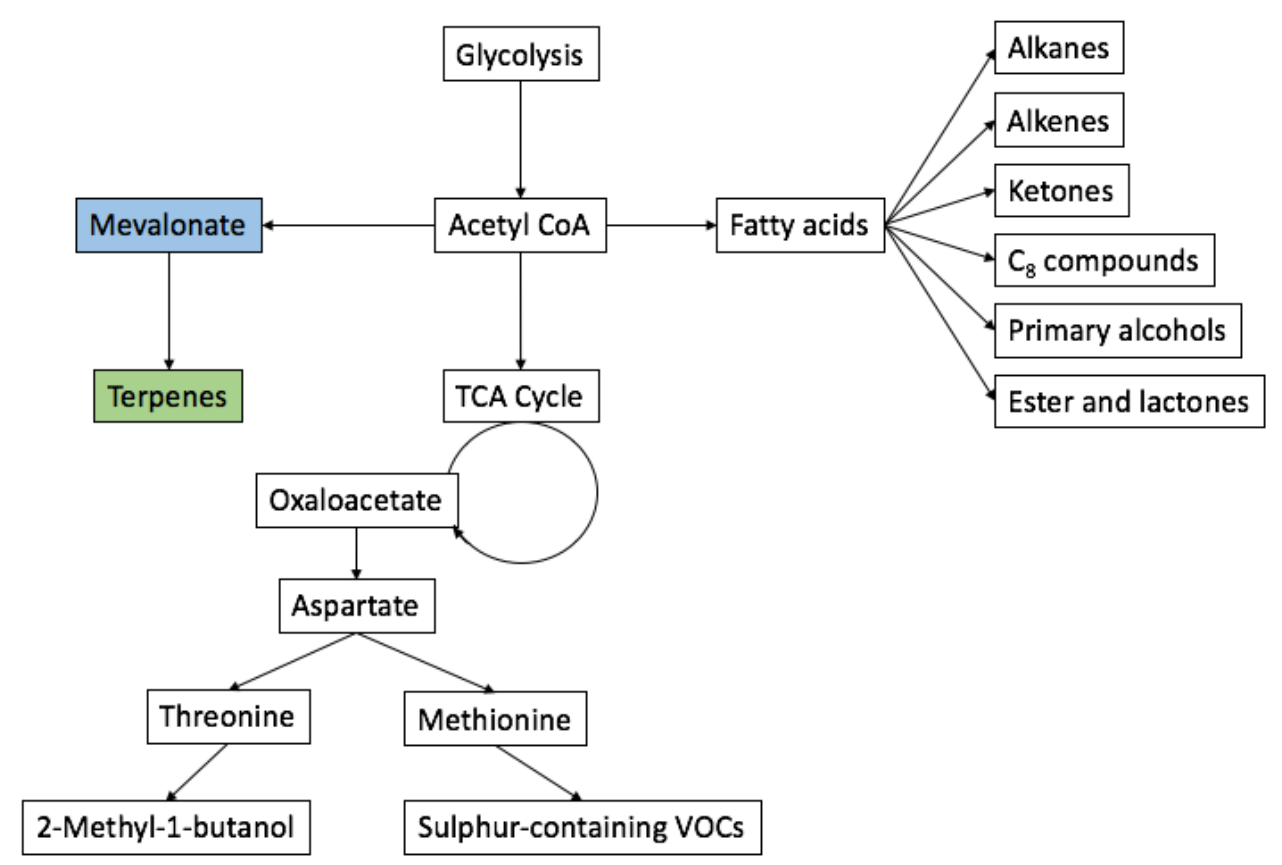

Figure 1: Products of the TCA cycle and MVA pathway in plants, stemming from glycolysis. Adapted from Schnürer et al. $(1999)^{14}$. 


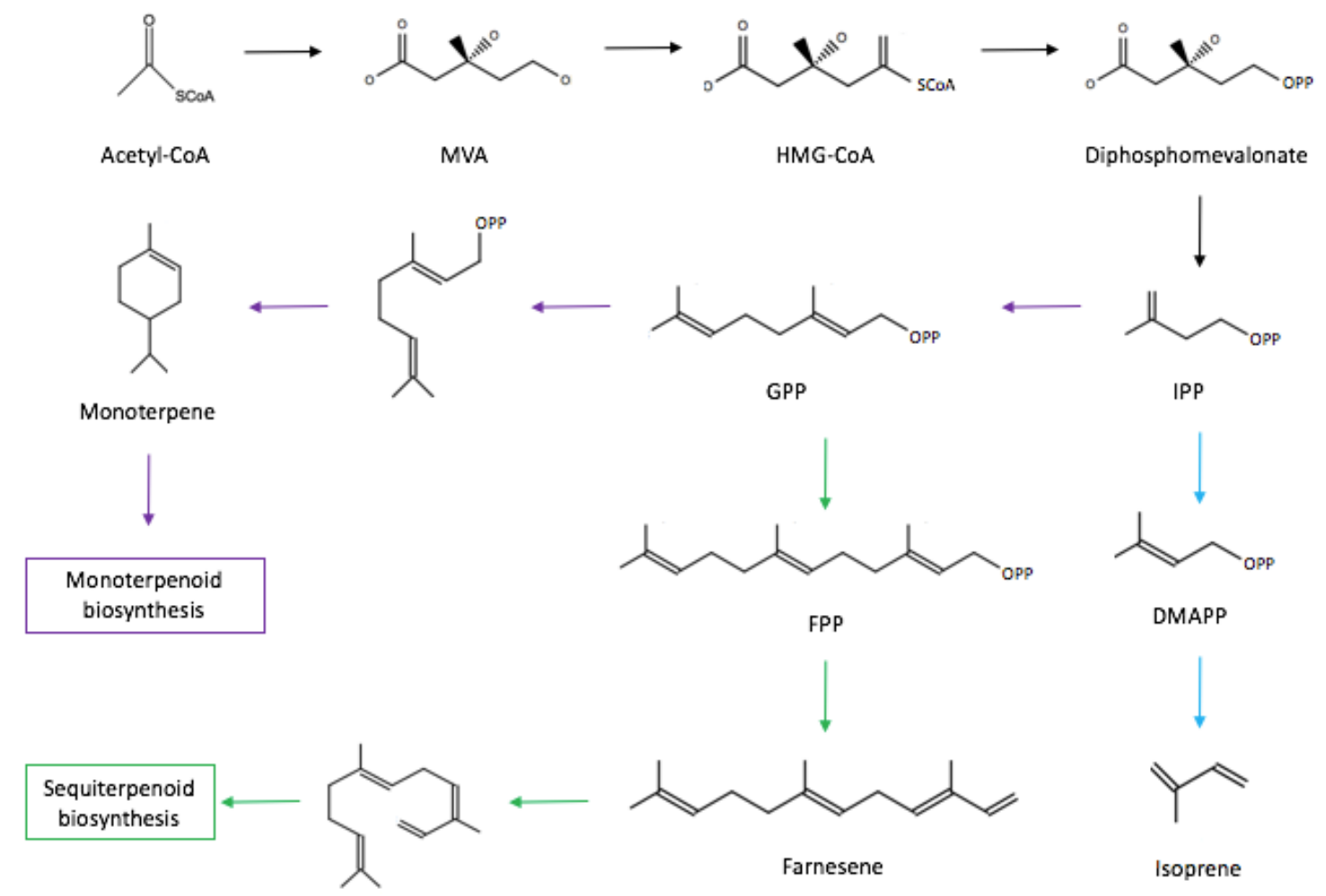

Figure 2: The MVA pathway in plants showing the creation of monoterpenes and sesquiterpenes.

Understanding the terpenes released by plants infected with certain pathogens benefits crop defense because it defines the biosynthetic pathways activated that cause harm or death to specific plants. The knowledge of terpenes can, in turn, be used to bolster plants' immunity against target pathogens, fungi, mold, and herbivores, resulting in a more robust agricultural defense system.

All pathogens and fungi produce VOCs ${ }^{12}$. Morath et al. (2012) labeled fungal VOCs "infochemicals" because of the amount of information they can provide about the organism, its metabolic pathways, and its interactions in nature ${ }^{12}$. Volatile organic compounds are unique for each fungal group and species, and can be used as biomarkers 
Table 1: The VOCs previously identified from trunk samples of avocado trees ${ }^{18-20}$.

\begin{tabular}{|c|c|c|}
\hline Compound & Variety & Reference \\
\hline$\alpha$-Humulene & Seedless Mexican, Simmonds, Melendez, Brooks Late & Niogret et al. $2011^{18}$, Niogret et al. $2013 b^{20}$ \\
\hline$\alpha-C o p a e n e$ & Seedless Mexican, Simmonds, Melendez, Brooks Late & $\begin{array}{l}\text { Niogret et al. } 2011^{18} \text {, Niogret et al. } 2013 \mathrm{a}^{19} \text {, } \\
\text { Niogret et al. } 2013 b^{20}\end{array}$ \\
\hline$\alpha$-Cubebene & Seedless Mexican, Simmonds, Melendez, Brooks Late & $\begin{array}{l}\text { Niogret et al. } 2011^{18} \text {, Niogret et al. } 2013 \mathrm{a}^{19} \text {, } \\
\text { Niogret et al. } 2013 \mathrm{~b}^{20}\end{array}$ \\
\hline Cadinene & Seedless Mexican, Simmonds, Melendez, Brooks Late & Niogret et al. $2011^{18}$, Niogret et al. $2013 \mathrm{a}^{19}$ \\
\hline$\beta$-Caryophyllene & Seedless Mexican, Simmonds, Melendez, Brooks Late & $\begin{array}{l}\text { Niogret et al. } 2011^{18} \text {, Niogret et al. } 2013 a^{19} \text {, } \\
\text { Niogret et al. } 2013 b^{20}\end{array}$ \\
\hline$\beta$-Cubenene & Seedless Mexican, Simmonds, Melendez & Niogret et al. $2013 a^{19}$, Niogret et al. $2013 b^{20}$ \\
\hline Alloaromadendrene & Seedless Mexican, Melendez & Niogret et al. $2013 a^{19}$, Niogret et al. $2013 b^{20}$ \\
\hline$\beta$-Pinene & Brooks Late, Melendez & Niogret et al. $2011^{18}$, Niogret et al. $2013 b^{20}$ \\
\hline
\end{tabular}




\begin{tabular}{|c|c|c|}
\hline Sabinene & Melendez & Niogret et al. $2013 b^{20}$ \\
\hline Monoterpene (RI 999) & Melendez & Niogret et al. $2013 b^{20}$ \\
\hline 3-Carene & Melendez & Niogret et al. $2013 b^{20}$ \\
\hline Monoterpene (RI 1052) & Melendez & Niogret et al. $2013 b^{20}$ \\
\hline Monoterpene (RI 1124) & Melendez & Niogret et al. $2013 b^{20}$ \\
\hline Monoterpene (RI 1290) & Melendez & Niogret et al. $2013 b^{20}$ \\
\hline$\delta$-Elemene & Melendez & Niogret et al. $2013 b^{20}$ \\
\hline Sesquiterpene (RI 1386) & Melendez & Niogret et al. $2013 b^{20}$ \\
\hline$\beta$-Elemene & Melendez & Niogret et al. $2013 b^{20}$ \\
\hline Sesquiterpene (RI 1409) & Melendez & Niogret et al. $2013 b^{20}$ \\
\hline (Z)- $\alpha$-Bergamotene & Melendez & Niogret et al. $2013 b^{20}$ \\
\hline
\end{tabular}




\begin{tabular}{|c|c|c|}
\hline Sesquiterpene (RI 1456) & Melendez & Niogret et al. $2013 b^{20}$ \\
\hline Sesquiterpene (RI 1470) & Melendez & Niogret et al. $2013 b^{20}$ \\
\hline Muurolene & Melendez & Niogret et al. $2013 b^{20}$ \\
\hline Sesquiterpene (RI 1508) & Melendez & Niogret et al. $2013 b^{20}$ \\
\hline Sesquiterpene (RI 1510) & Melendez & Niogret et al. $2013 b^{20}$ \\
\hline$\beta$-Bisabolene & Melendez & Niogret et al. $2013 b^{20}$ \\
\hline$\delta$-Cadinene & Melendez & Niogret et al. $2013 b^{20}$ \\
\hline (E)- $\alpha$-Bergamotene & Melendez & Niogret et al. $2013 b^{20}$ \\
\hline Sesquiterpene (RI 1436) & Melendez & Niogret et al. $2013 b^{20}$ \\
\hline
\end{tabular}


through chemotyping ${ }^{10}$. Researchers have thus far identified over 300 VOCs from various fungi $^{5,12,17}$, a list which is continually expanding, and many of which are terpenes. It is well known that fungal VOCs are biologically dynamic, and are therefore affected by various factors, such as the media on which it is grown, the duration of incubation, the type of nutrients it can access, the environmental temperature in which it is grown, and various other environmental conditions ${ }^{9,12,21-23}$.

While limited peer-reviewed literature exists identifying terpenoids and VOCs of individual fungal VOCs' effect on specified plants, there is a small amount of literature that identifies the VOCs produced from Raffaelea lauricola hosts $^{18-20,24}$ (Table 1). Only two studies have focused on the headspace of the fungus itself $\mathrm{f}^{25,26}$, and only one has identified the VOCs of hosts actually infected with R. lauricola ${ }^{24}$. Kuhns et al. (2014) identified four VOCs above four-day-old $R$. lauricola samples, one of which was also present in the blank. These compounds were: ethyl acetate (which was also present in the blank), ethyl alcohol, isobutyl alcohol, and isoamyl alcohol. On the basis of these results, the authors determined that $R$. lauricola increases the "attractiveness" of a tree for additional attacks by the RAB vector $^{26}$. The results of the Simon et al. (2017) studies identifying odor profiles of $R$. lauricola, and healthy versus infected avocado trees are presented in this report ${ }^{24,25}$.

\subsection{Global Biotic Homogenization of Ambrosia Beetles}

Global biotic homogenization refers to the process by which invasive species move across the earth. As global human populations continue to travel and trade, alien species are introduced to new ecosystems, whether on purpose or by accident. Shipping pallets and crates are often sources for the introduction of invasive insects and pathogens whose hosts 
are woody tissues. Some reasons that new species are so successful in new environments include the lack of natural enemies, the development of new symbiotic associations, effective predators in new ecosystems, artificial or disturbed habitats that make an ecosystem favorable to new species, and invasion by highly adaptable species $^{27}$.

Invasive species in the United States cause approximately $\$ 120$ billion in damages and losses each year as a result of environmental and agricultural damages and control $\operatorname{costs}^{27}$. While there are nearly 750,000 native species in the US, there are almost 50,000 alien species, many of which threaten native flora and fauna ${ }^{27}$. While $42 \%$ of threatened and endangered species under the Endangered Species Act in the United States are listed primarily because of competition with or infection by invasive species, some regions of the world consider $80 \%$ of their threatened and endangered species to be listed as a result of translocated species. For example, alien plant pests and pathogens cause an estimated $\$ 21$ billion in losses each year with an additional $\$ 0.72$ billion spent on fungicides to counteract potential loss in the United States ${ }^{27}$. These numbers are continuously increasing as the rate of global biotic homogenization increases.

Ambrosia beetles are an exceptional example of translocated species because of their symbiotic partners, which are fungi carried in specialized organs of the exoskeleton near the mouth of the beetle called mycangia. Ambrosia beetles are wood-boring insects that, together with their symbiotic fungi, are emerging as currently uncontrollable threats to forest ecosystems, fruit industries, and timber industries throughout the world. The symbioses offer several advantages to both partners: the beetles are provided with food, the fungi can pre-digest the tree tissue, and the fungal spores rely on the beetles for transport to new hosts. The human transport of these symbiotic species means that they are 
being introduced at a faster rate than any other introduced forest pest. For example, the closely-related bark beetles constitute less than $0.2 \%$ of insect species on earth, but over half the interceptions at ports-of-entry in the $\mathrm{US}^{28}$. Because of how quickly these insects are spreading, and how little is understood, there are significant gaps in literature explaining ecology, epidemiology, and management of the effects of ambrosia beetles ${ }^{28,29}$. As more research can characterize the beetles and their symbionts, the ability to intercept species prior to entrance and prevent or control the spread once translocated is greatly enhanced.

\subsection{Laurel Wilt Causes and Disease Cycle}

Laurel wilt is a disease caused by the redbay ambrosia beetle (RAB), or Xyleborus glabratus, and its symbiont, the fungus $R$. lauricola. It is a novel disease in the United States that was introduced when the vector RAB was transported to Georgia from Southeast Asia in infected wood shipping pallets in the early 2000s. Genetic uniformity in tested samples of $R$. lauricola recovered in the United States, in contrast to high diversity from recovered samples in Japan and Taiwan, has lead researchers to conclude that introduction was a single event ${ }^{30,31}$. Since its introduction into the country, the fungus has spread from its point of origin in Georgia to ten states: Virginia, North Carolina, South Carolina, Florida, Alabama, Mississippi, Louisiana, Arkansas, and Texas, infecting nine members of the Lauraceae ${ }^{32}$, including avocado trees (Persea americana) ${ }^{1,30,33-37}$ (Figure 3). 


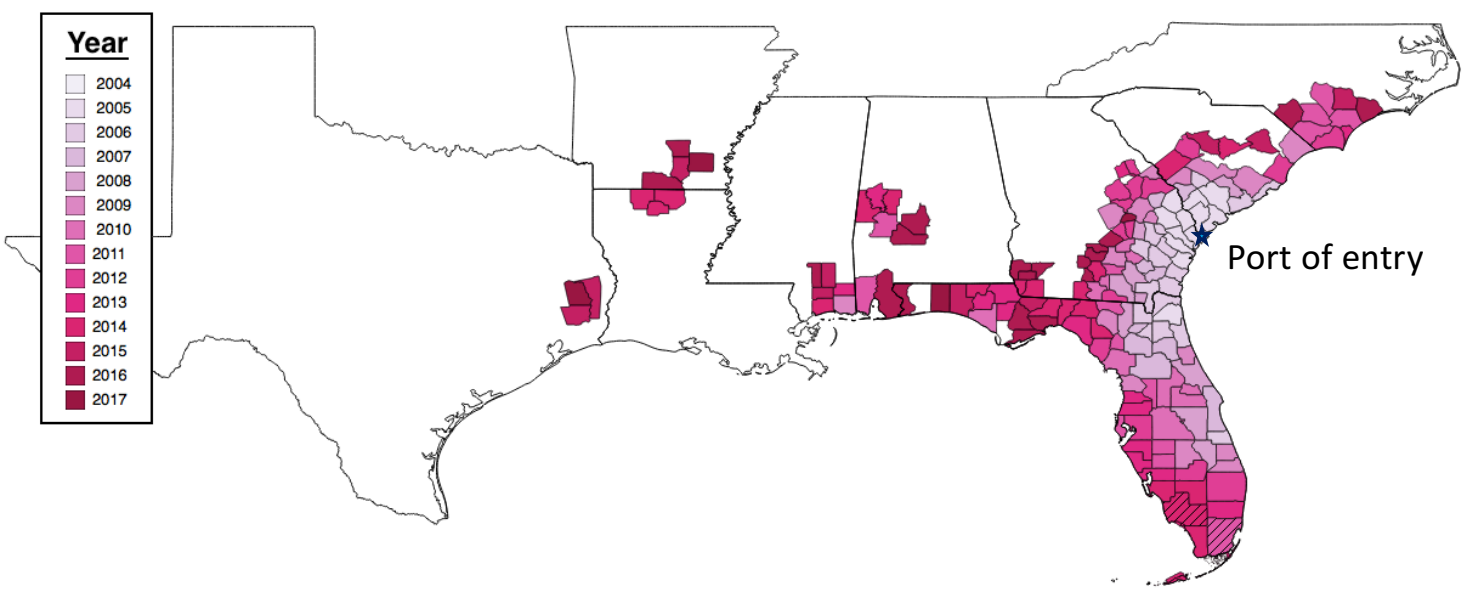

Figure 3: Map of the spread of $\boldsymbol{R}$. lauricola since entry into the United States (as of April 2017). Black stripes indicate avocado growing regions. *Affected counties in Virginia not indicated.

The RAB was named after its preferred host tree, the redbay tree. As the redbay tree began to die out, the RAB began using other members of the same phylogenic subset as hosts, including the avocado tree. In its native range, the RAB colonizes dead trees because they are more vulnerable and conducive to insect life than live trees. Live trees produce volatiles, chemicals, latex, and resins as protection from colonization. Once the trees die, these defenses dissipate, allowing insects to more easily access nutrients ${ }^{1,23}$. However, in the United States the RAB colonizes living trees. There are several theories that explain why the beetles in different regions have different host preferences. The first theory, called permissive choice, suggests there may be differences in the beetles in Asia versus those in the United States, while the second theory, called odor mismatch, says the differences may be in the host ${ }^{1}$. In permissive choice theory, there are genetic differences in the $\mathrm{RAB}$ in native and non-native regions that allow them to be attracted to slightly different odors ${ }^{1}$. However, permissive choice theory is challenged because the RAB reproduces almost clonally, reducing genetic variation. The more likely theory, odor 
mismatch, states that the differences are in the trees' odors because they are from a narrow phylogenic subset ${ }^{1,38}$. Possibly, dead trees in the native regions have similar odors to live trees in the non-native regions; the $\mathrm{RAB}$ does not recognize that it is boring into a live tree. Once the beetle reaches the xylem and realizes the tree is still alive, the RAB leaves the tree in search of a dead host ${ }^{1,38}$. However, the live tree is inoculated with $R$. lauricola, so the tree weakens and is susceptible to colonization by other beetles. Other RABs and other ambrosia beetles are thus attracted to the injured trees, and often accidently begin carrying R. lauricola spores along with their unique symbiotic partners, compounding the spread ${ }^{39-41}$.

Once a tree is inoculated by a beetle, symptoms occur within four weeks and most trees die within six weeks. Mayfield et al. (2008) tracked and compared the progress of the laurel wilt disease within redbay and avocado trees under greenhouse conditions. Redbay trees wilted within 2-3 weeks of inoculation, while avocado trees wilted within 2-4 weeks. The research confirmed that the laurel wilt pathogen efficiently kills or damages these members of the Lauraceae ${ }^{34}$. A similar experiment was conducted by Ploetz et al. (2011) to compare the progress of the disease under field and greenhouse conditions. It was reported that symptoms were seen within 10-14 days of inoculation ${ }^{36}$, even sooner than Mayfield et al. (2008) had originally reported ${ }^{34}$.

The disease cycle is shown in Figure 4. Once a RAB bores into a tree, it builds a fungal garden to farm the fungus used as food. These fungal gardens are found in the xylem of the tree, which is the vascular portion of the trunk that moves water. The fungus gains nutrients from the tree, and as the fungus grows, the beetle and its larvae feed on the fungus $^{1,42}$. Once a female beetle is full grown, she leaves the host tree to find another in 
which to build a fungal garden. Once the new fungal garden is established, she lays eggs, and the disease cycle begins anew. After a tree is initially inoculated, the fungus spreads in the tree's vascular system, moving downward into the roots. The movement is an issue because trees in commercial avocado groves are planted close enough to each other that root grafting occurs. In root grafting, the roots of nearby trees grow in overlapping and random patterns, meaning the fungus can spread from tree to tree without beetle intervention. Essentially, when one tree in a grove is infected, the entire grove is in danger.

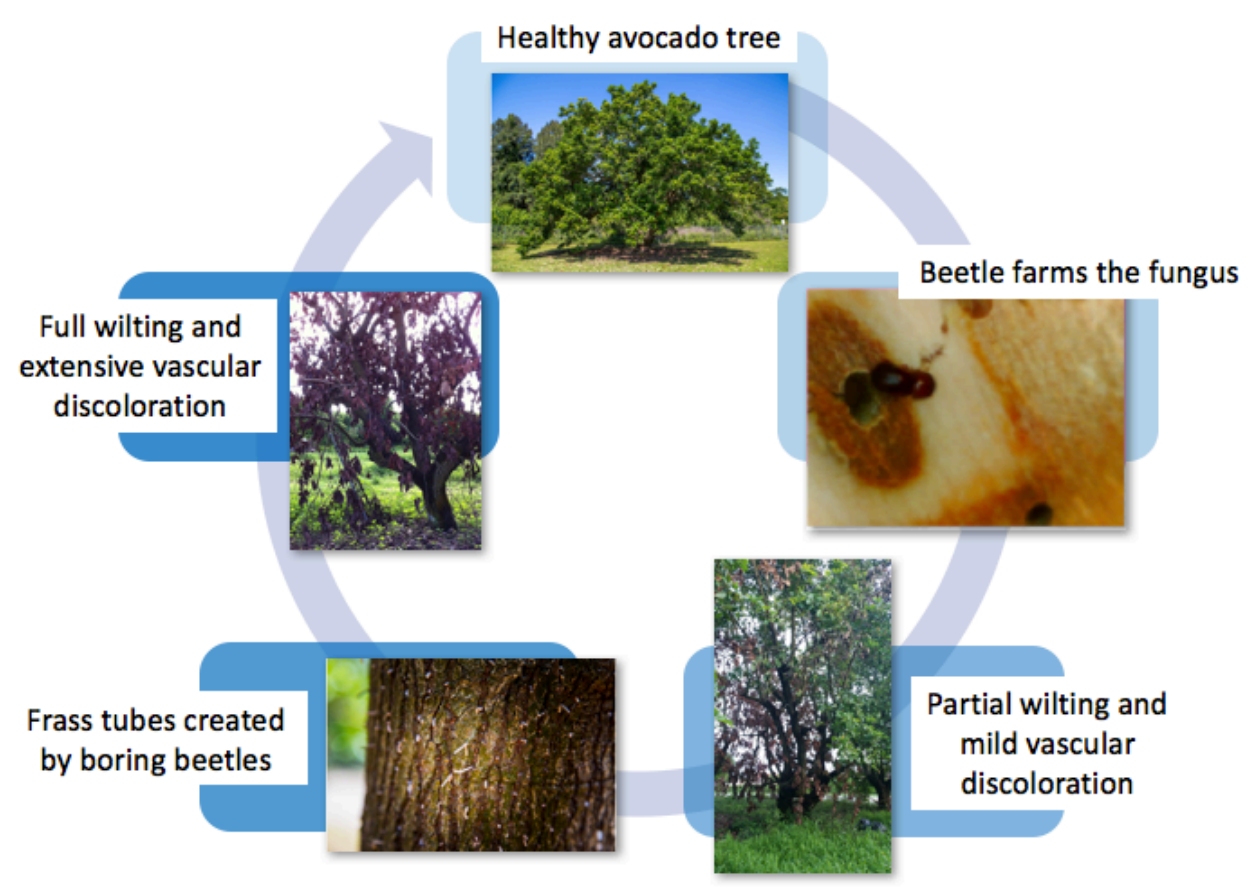

Figure 4: The disease cycle of laurel wilt.

The mechanisms of tree death as a result of laurel wilt are poorly understood because $R$. lauricola is the first ambrosial fungus that is known to be aggressive ${ }^{19}$. The best hypothesis, known as tree suicide, states that the pathogen itself has a low virulence, so the 
tree dies from its own response rather than from the pathogen itself ${ }^{35}$. The host tree swells the xylem's vessels to stop the spread of the pathogen. Additionally, the tree begins to produce gums and tyloses to occlude the vessels and physically halt movement of the fungus ${ }^{1,33,34,37,43}$. However, this inadvertently stops the transport of nutrients, water, and oxygen necessary to keep the tree itself alive $e^{1,33,34,37,43}$. Wood begins to stain and leaves wilt, eventually progressing to death. Tree deaths have not been reported in the RAB's native regions ${ }^{36}$, suggesting that trees in the United States have developed an exaggerated response or have no capacity to fight the fungus, resulting in tree suicide.

\subsection{Treatment of Laurel Wilt Disease}

Currently, proper sanitation dictates that all avocado trees in Miami-Dade County identified as having trees infected with laurel wilt be removed from the ground, chipped, burned, and left in place. There is no current fungicide that can kill $R$. lauricola. There is, however, an EPA approved fungicide that can be used as a pre-treatment to help prevent infection in commercial groves since it leaves no residue in fruit. This fungicide is called Tilt ${ }^{\circ}$ (propiconazole) ${ }^{106,107}$. Propiconazole is a demethylation inhibitor, meaning they inhibit the production of sterols, which are necessary for the development and function of cell walls in fungi ${ }^{106,107}$. Tilt ${ }^{\circledR}$ provides the best protection, and is generally applied through a technique called macroinfusion, which applies large volumes of the fungicide to flare roots of the target trees ${ }^{106,107}$. However, it is both time consuming and cost-prohibitive for most farmers, so it is not largely utilized.

Twenty-five other fungicides have been tested for in vitro inhibition of $R$. lauricola by Ploetz et al. (2011), though the results were never published ${ }^{46}$. A recent study by Ploetz 
et al. (2017) tested four fungicides, including Tilt ${ }^{\circledR}$, for efficacy and detectability in trees and fruit ${ }^{46}$. Additionally, several nutritional supplements and systemic acquired resistance (SAR) products were examined. The SAR products are essentially a way of enhancing a plant's innate responses to a pathogen, like an immune system. Macroinfusion resulted in less disease, but more residue; however, all fungicides were effective at reducing laurel wilt development. When present at all, no tested fruit exceeded the safe levels of residue. All nutritional and SAR treatments were ineffective ${ }^{46}$.

Another recent study by Carrillo et al. (2015) evaluated three entomopathogenic fungi as biological control methods for the $\mathrm{RAB}^{39}$. Entomopathogenic fungi essentially act parasitic towards insects and can kill them. All three tested biological control agents killed the $\mathrm{RAB}$, though they did so with different timelines for survival and efficacy. Depending on the fungus used, the beetles survived 3-5 days, which means they could still effectively bore into trees, but could not lay eggs ${ }^{39}$ (it takes approximately 7 days for the RAB to produce a brood) ${ }^{108}$. These methods show potential for use in commercial and residential avocado groves.

Since so many beetles are known to carry $R$. lauricola, insecticides have also been explored as a method of prevention and control. Twenty different systematic and direct insecticides have been studied ${ }^{109,110}$. Tested insecticides had much shorter periods of effective suppression than had been previously noted, which was attributed either to the avocado tree bark the South Florida subtropical conditions, or ambrosia beetle tolerance ${ }^{110}$. Suppression lasted no longer than a few days. Additionally, persistence of the chemical residues was low, possibly influenced by rainfall or sunlight ${ }^{110}$. While reduction in the number of entrance holes could be reduced, it was not eliminated ${ }^{109}$. Each study suggests 
that multiple treatments of a grove would be required each year, which would not necessarily be economically feasible.

Other methods of control for laurel wilt include trapping and repelling RABs. Trapping involves the use of lures to attract and contain beetles. Effective traps assist in detection and control of beetles ${ }^{42,111-119}$. Initial research identified commercial manuka and phoebe oil traps to be the most effective $\mathrm{e}^{26,30,31,112,114,117}$. However, more recent studies have shown that traps containing copaene are the most effective attractant for $\operatorname{RABs}^{42,119}$. Copaene is also the VOC produced by Lauraceae hosts that attracts $\operatorname{RABs}^{42,112,119}$. Additionally, this volatile is released in high concentrations from the trunk, where beetles attack for colonization, and in lower amounts from the branches and leaves ${ }^{120}$. Another volatile, eucalyptol, is also an attractant for RABs and may show potential for use in management techniques ${ }^{121}$. More recently, cubeb oil traps have been to be acceptable replacements for Manuka oil and phoebe oil traps, which are no longer an option either because of high price or low availability ${ }^{118,122}$. In an evaluation of traps themselves, it was discovered that RABs fly between 1600 and 1800 hours at heights of $35-100 \mathrm{~cm}$ above the ground $^{111}$, with seasonal peaks in March/April and September/October ${ }^{111113}$. Additionally, color does not appear to be a cue for RABs, but the age of the traps does have an effect, with fresh traps being significantly more effective ${ }^{111,113}$.

Hughes et al. (2016) evaluated the efficacy of certain volatiles for use as repellants of the RAB. Verbenone and methyl salicylate were chosen as the best repellants ${ }^{123}$. Verbenone is produced by mycangial fungi of certain bark beetles, while methyl salicylate is a natural SAR compound previously linked to herbivore repellency. Verbenone was the 
most effective repellant. While further research is suggested, the study suggests that beetles could be repelled from the areas their hosts occupy using select volatile compounds ${ }^{123}$.

\subsection{Impact of Laurel Wilt in South Florida}

In Miami-Dade County, which contains $98 \%$ of Florida's $\$ 55$ million avocado production, laurel wilt disease has killed over 12,000 commercial trees since it first appeared in the county in $2009^{44}$. Miami-Dade County also contains over 250,000 privately owned avocado trees, all of which are at risk of laurel wilt ${ }^{44}$. Once laurel wilt has been detected in an avocado tree, whether commercial or residential, Miami-Dade County ordinance states that the grove owner has 20 days to remove the infected tree and destroy $\mathrm{it}^{45}$. The disease has been associated with mortality in at least 300 million wild redbay trees in forests and natural ecosystems in the southeastern region of the United States ${ }^{30,46,47}$.

\subsubsection{Economic Impacts}

Avocado crops in the Unites States produce over $\$ 1.3$ billion (value-added) that is threatened by the continued spread of laurel wilt ${ }^{48}, \$ 356$ million of which is attributed to the total baseline value of Florida avocado groves ${ }^{33}$. There are many considerations when examining economic impacts, including: the cost of tree removal and disposal, loss of property value, increased administrative and management costs, revenue loss for fruit, unprocessed wood or nursery businesses, and fire hazards associated with standing dead trees in natural areas ${ }^{32}$. Specific to avocado crops, the value of a mature tree is estimated to be $\$ 500$ considering that the farm gate price is $\$ 0.45 / 1 \mathrm{~b}$. However, the application of certain treatment options (including the material, the labor, and the machinery) can cost 
\$333/acre per year. Additionally, increased monitoring costs would be about $\$ 270 /$ acre $^{33}$. Many farmers and grove owners consider this high cost of preventative measures such as treatment and monitoring to be uneconomical. Areas such as this with little or no disease management allow for rapid spread of the disease and increased economic losses ${ }^{48}$.

\subsubsection{Ecological Impacts}

Fewer than half of the 750,000 species in the United States are characterized ${ }^{27}$, which makes identifying the full extent of ecological impacts for any given alien species very difficult. However, certain aspects can be determined. For $R$. lauricola, those impacts include harming and further exposing many threatened or endangered species. The fungus R. lauricola has been identified in fourteen species thus far, five of which have not yet been identified in the wild (Table 2). Three of the tree species affected by the laurel wilt disease are already listed on the federal Endangered Species Act, Pondspice, Pondberry, and Pepperleaf Sweetwood. Pondspice ${ }^{52.58}$ is a threatened species, while Pondberry ${ }^{52}$ and Pepperleaf Sweetwood ${ }^{56}$ are endangered. Additionally, viñátigo ${ }^{54}$, a Spanish member of the Lauraceae, is endangered according to the International Union for Conservation of Nature (IUCN) Red List of Endangered Species. Raffaelea lauricola has the potential to eradicate each of these species ${ }^{32,49-60}$. Though redbay trees are the primary attractant of RABs in the United States, they have been observed attacking several other species of tree $\mathrm{s}^{32,34,46,51,53}$. In addition, other beetles can carry $R$. lauricola, which further endangers members of the Lauraceae, and, indirectly, the species which rely on such trees ${ }^{39}$.

Another major concern is the butterfly Palamedes Swallowtail, Papilio palamedes, an endangered insect whose primary larval host is the redbay tree ${ }^{61}$. The redbay tree has a 
mortality rate above $95 \%$ with extremely rapid symptom progression. Previous studies have stated that trees may die within eight weeks ${ }^{51}$. Because the Palamedes Swallowtail relies almost entirely on the canopy of redbays, the distribution of the two species mirrors each other. While there are records of the butterfly living on other members of the Lauraceae, notably the camphor tree, larval performance and survival rate of the adults on those tree is far lower ${ }^{61,62}$. Though less studied, the leaf miner, Phyllocnistis subpersea, is also primarily dependent on redbays for food ${ }^{63,64}$. With dwindling numbers of redbay trees, populations of Palamedes Swallowtails, the flowers they pollinate, and other such dependent species are in danger.

In a recent assessment, Evans et al. (2014) detailed the ability of redbay forests in the southeast United States to recover their canopy role. They found that new sprouts are not able to persist long enough, likely because of consumption by deer or other herbivores. Additionally, there is a small seed pool from the devastation that laurel wilt has caused. With the disappearance of redbay trees, there are several associated detrimental effects of canopy and subcanopy loss. Light penetration through the thinning canopy will affect understory thermal regimes, altering the ground flora structure and productivity, which in part determine the fauna make up and efficiency ${ }^{63}$. Subcanopy density changes will alter the habitat for nesting birds. Additionally, the changes in litterfall and detritus will modify nutrients and nutrient cycling, which influence soil biota ${ }^{63}$. In the Everglades specifically, peat accretion rates could fall, which could cause increased flooding in tree islands and cause instability and nutrient deficiencies that could severely alter those fragile ecosystems ${ }^{65-67}$. 
Table 2: Hosts of R. lauricola by the year and location of identification ${ }^{32,49-60}$.

*Endangered species. $\uparrow$ Non-native species used for agriculture or ornament. $₫$ Not yet infected in the wild.

\begin{tabular}{|c|c|c|c|}
\hline & Host & First Identified (year, location) & Reference \\
\hline 1 & Redbay (Persea borbonia) & 2002, Port Wentworth, GA & Fraedrich et al. $2008^{51}$ \\
\hline 2 & Pondspice (Litsea aestivalis)* & 2006, Beaufort, SC & $\begin{array}{l}\text { Fraedrich et al. } 2011^{52} \text {, } \\
\text { Hughes et al. } 2011^{58}\end{array}$ \\
\hline 3 & Pondberry (Lindera melissifolia)* & 2006, Effingham, GA & Fraedrich et al. $2011^{52}$ \\
\hline 4 & Spicebush (Lindera benzoin) $\ddagger$ & 2006, research & Fraedrich et al. $2008^{51}$ \\
\hline 5 & Avocado (Persea americana) $\dagger$ & 2007, Jacksonville, FL & Mayfield et al. $2008^{55}$ \\
\hline 6 & Camphortree (Cinnamomum camphora) $\dagger$ & $\begin{array}{l}\text { 2007, McIntosh and Glynn Counties, } \\
\text { GA and Baker County, FL }\end{array}$ & $\begin{array}{l}\text { Smith et al. } 2007^{57} \\
\text { Fraedrich et al. } 2015^{53}\end{array}$ \\
\hline 7 & Sassafras (Sassafras albidum) & $\begin{array}{l}\text { 2007, Effingham, Evans, Bulloch, } \\
\text { Liberty, and McIntosh Counties, GA }\end{array}$ & $\begin{array}{l}\text { Fraedrich et al. } 2008^{51} \text {, } \\
\text { Cameron et al. } 2008^{49}\end{array}$ \\
\hline
\end{tabular}




\begin{tabular}{|c|c|c|c|}
\hline 8 & California laurel (Umbellularia californica) $\ddagger$ & 2008, research & Fraedrich $2008^{50}$ \\
\hline 9 & Silk bay (Persea humilis) & 2011, Highlands County, FL & Hughes et al. $2012^{59}$ \\
\hline 10 & Swamp bay (Persea palustris) & 2011, Everglades National Park, FL & Fraedrich et al. $2008^{51}$ \\
\hline 11 & Pepperleaf Sweetwood (Licaria triandra) ${ }^{*} \ddagger$ & 2012, research & Ploetz and Konkol $2013^{56}$ \\
\hline 12 & Viñátigo (Persea indica) $\dagger \ddagger$ & 2012, research & Hughes et al. $2013^{54}$ \\
\hline 13 & Bay laurel (Laurus nobilis) $\dagger$ & 2013, Gainesville, FL & Hughes et al. $2014^{60}$ \\
\hline 14 & Lancewood (Nectandra coriacea) $\ddagger$ & 2015, research & Hughes et al. $2015^{32}$ \\
\hline
\end{tabular}


While redbay trees and avocado trees are the most well studied of the 14 affected species, the loss of other species will also have detrimental effects to the flora, fauna, and biota that interact with and rely on members of the Lauraceae. For example, the ecological impacts of sassafras tree loss have not been studied, but the species is highly populous in the southern piedmont and Appalachian Mountains regions. Therefore, loss could affect composition and productivity of flora and fauna in similar manners to redbay tree loss effects in the Coastal Plain and maritime hammocks ${ }^{32}$. Additionally, other more aggressive invasive plants could replace redbay trees in these essential Everglades and Appalachian ecosystems ${ }^{32,66,67}$.

\subsubsection{Cultural Impacts}

The spread of laurel wilt and decline in species affects not only the economy and ecology of the Southeastern region, but the culture as well. Redbay tree wood has been used locally for boatbuilding and cabinetry ${ }^{68}$. Additionally, redbay leaves can be used as a seasoning for food ${ }^{68}$. Other members of the Lauraceae, such as camphortree or bay laurel are used in ornamental yard displays ${ }^{32}$. One obvious cultural impact of avocados is food, namely guacamole. For example, Americans consume an estimated 104.9 million pounds of avocados on Super Bowl Sunday ${ }^{69}$. Also, the \#savetheguac campaign has taken on a life of its own in social media outlets, such as Facebook, Instagram, and Twitter.

Furthermore, sassafras, California laurel, redbays, and swamp bays are a center of Seminole, Miccosukee, Creek, and Choctaw Native American Tribal life ${ }^{70}$. More than 90\% of traditional medical practices require redbay (known as tu lee by the Seminole Tribe, 
toole by the Miccosukee Tribe, and toola by the Creek Tribe) ${ }^{71}$, as well as cultural celebrations, purification rites, and ceremonies. It is used for many things, from treating fevers to arthritis. The Creek and Choctaw tribes also used swamp bay trees for medicinal purposes such as fever and dropsy ${ }^{72}$.

\subsection{Laurel Wilt Detection Methods}

Laurel wilt disease is detectable through visual inspection. Using this method, wilting leaves, discolored wood, and frass (fine wood produced by wood-boring insects) are used as visual confirmation. The trees are then removed by chipping and burning. However, once the disease is detectable visually, the tree cannot be saved, and the fungus has likely spread to the surrounding trees via root grafting. It is therefore essential to develop non-visual standoff detection methods so that groves can be screened for the disease before tree removal becomes necessary.

There are only a handful of methods used to detect trees affected with laurel wilt

disease. These methods include either aerial imaging ${ }^{73-77}$, canine detection ${ }^{24,47,78}$, or a combination of the two ${ }^{86}$. Aerial imaging can be done using helicopters or drones to fly over avocado groves and identify areas of infection or concern. Canines can be run in suspected groves to identify specific affected trees. These two detection efforts can be used individually, but they are more effective when used in tandem. In this case, aerial imaging is used to identify injured areas of a grove, and canines are then used to identify specific trees and ground truth. Using these methods, infected trees can be treated or removed, thereby protecting the remainder of the grove. 


\subsubsection{Aerial Imaging}

There are several methods of aerial imaging possible for the detection of laurel wilt disease in avocado trees. These methods use visible symptoms of the leaves to identify injured trees. One method involves simple visual inspection from a helicopter followed by ground visits to collect wood samples for testing ${ }^{73-77}$. However, this method generally takes about two weeks to confirm infection and is cost prohibitive ${ }^{76}$. A faster, nondestructive technique is visible-near infrared reflectance spectroscopy (VIS-NIR), which collects data on wavelengths from $400 \mathrm{~nm}$ to $950 \mathrm{~nm}$. Chlorophyll adsorption and reflectance affect pigment concentration, which is perceptible in the visible range $(450-760 \mathrm{~nm})$, while other factors such as physiological structure, condition of the cell wall, water content, surface roughness, and stoma activity may alter light penetration through the leaf, which can be detected in the near infrared (NIR) range $(760-2500 \mathrm{~nm})^{77}$. The VIS-NIR images can be collected using specialized filters on handheld cameras in low altitude helicopter surveys over suspected commercial avocado groves. Using the collected data along with statistical analyses, a vegetation index, such as the Red Green Ratio Index (RGRI), can be used to classify images ${ }^{73-77}$. These wavelengths are shown in Figure 5.

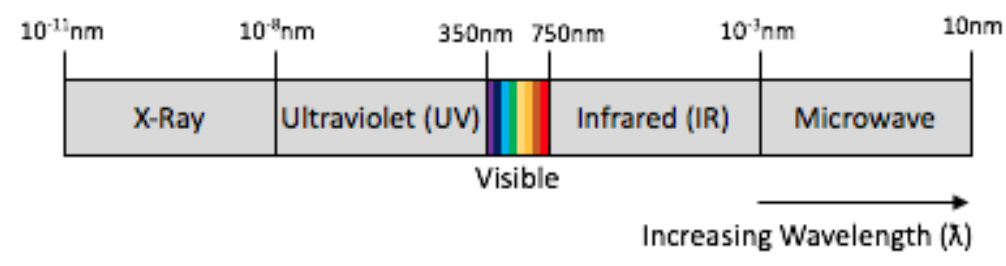

Figure 5: Diagram showing VIS-NIR wavelengths used to identify injured trees.

The symptoms of laurel wilt often resemble those of other diseases or avocado tree stressors, such as freeze damage, Phytophthora root rot, Verticillium wilt, lightning, fruit 
stress, salinity damage, or drought stress. However, using VIS-NIR, these various diseases can be distinguished. A major challenge, though, remains that leaves are asymptomatic for a period at the beginning of infection, and can therefore be misclassified as healthy ${ }^{76,77}$. The most useful vegetation indices are the Normalized Difference Vegetation Index (NDVI) and the spectral reflectance index (SRI) since they are sensitive to chlorophyll adsorption and reflectance, which changes with leaf necrosis ${ }^{76}$. De Castro et al. (2015) suggested a 4-class system for classification to improve on the 2 -class system in use ${ }^{73}$. The classification would rank images on a scale of healthy versus early, intermediate, and late stages of infection, and would improve accuracy ${ }^{73}$.

While helicopters are effective, they are also expensive. Unmanned aerial vehicles, or drones, have been used to address the issue of cost. Drones utilize the same methods of a modified camera in conjunction with vegetation indices to classify infected and healthy trees. Drones can carry 10 pounds of equipment and cover up to 10 acres in 15 minutes of flight time $^{78}$ (Figure 6). The most effective indices found are Normalized Pigment Chlorophyll Index (NPCI), Green Difference Vegetation Index (GDVI), and Excess Red (ExR), which achieved classification with an accuracy of $83.23 \%{ }^{79}$. 


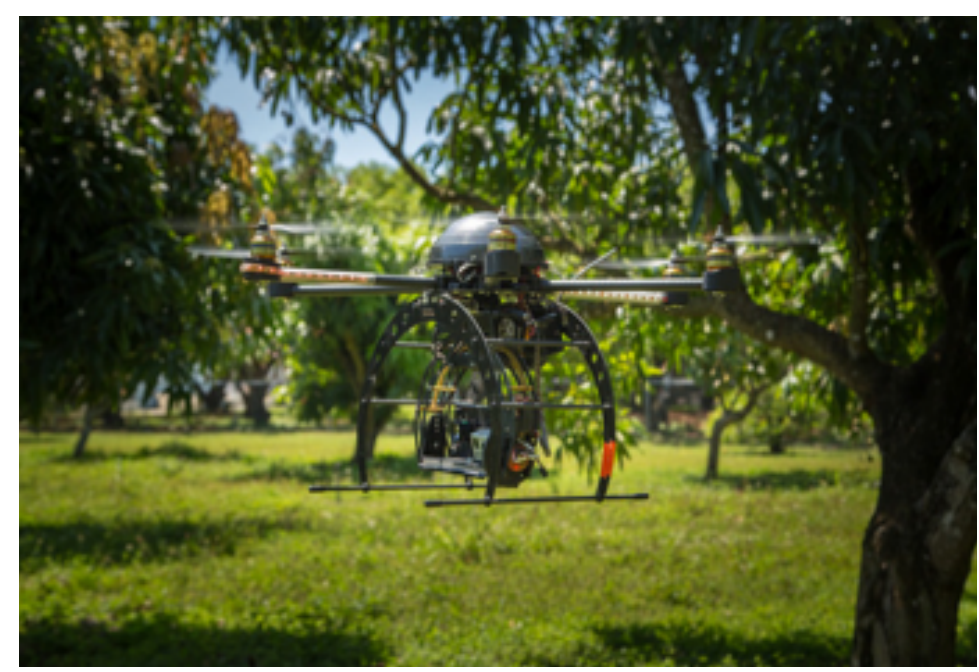

Figure 6: Drone fitted with camera used to capture aerial images of avocado groves.

\subsubsection{Canines}

As aerial imaging continues to improve, the challenge of distinguishing early infection from healthy trees remains. Canines, however, can fill this gap. They are sensitive, selective, and rapid onsite detectors that scent-to-source despite the presence of large amounts of background odor. They are also extremely mobile detectors that can search large amounts of area in many types of terrain. Canine substance detection is a diverse field, including traditional applications such as narcotics, explosives, currency, firearms, human scent, human remains, and ignitable liquid residues as well as more recent medical and environmental applications ${ }^{81-88,95-103}$. Within environmental detection, canines have been used to find scat and insects, for conservation surveys or eradication, for border protection, and for environmental research, including the identification of molds, bedbugs, termites, wildlife scat, poaching products, plants and agricultural products, and endangered or invasive species ${ }^{82-85}$. The first documentation of canines for use in environmental detection was in the 1890s in New Zealand, when dogs were trained to locate kiwis and 
other birds for relocation ${ }^{80}$. Through the 1930s and 1960s, dogs were used by biologists to find specimen for study, tagging, or museum displays ${ }^{81}$. Environmental detection canines have continued to gain popularity through the 1990s and into today, when they were used to help collect information of various rare or invasive species ${ }^{82-85}$. There are currently three trained and certified canine detection teams in the country that can identify laurel wilt disease before the appearance of visible or VIS-NIR detectable symptoms ${ }^{47,78,86}$.

To identify any target, canines detect odorants, which are molecules that can be perceived by the olfactory receptor proteins. An odorant is not necessarily the chemical of the actual target, but is associated with it by the canine. Once an odorant enters the nostrils, it travels to the olfactory mucosa, which transports it to the olfactory receptor. These receptors activate neurons in the olfactory bulb of the brain and send a signal to the cortex, where it is perceived and a reaction is determined ${ }^{87}$. Each odorant produces a unique combination of signals depending on the individual receptors that recognize it. The unique pattern of signals is what gives canines their incredible selectivity and discriminatory power. Their sensitivity can be attributed to the size of the olfactory epithelium, which is the portion of the mucosa containing neurons expressing the olfactory receptors. It covers an area of $70-170 \mathrm{~cm}^{2}$, depending on the breed ${ }^{88}$. Humans, on the other hand, cover only about $5 \mathrm{~cm}^{2}{ }^{88,89}$. Indeed, despite varying sizes between breeds, the canine olfactory epithelium can express 20 times more odor receptors than that of humans ${ }^{90}$.

To be perceived as an odorant, a molecule must either be volatile or enter the atmosphere through another means, such as attaching to a particle of dust. Dogs can force an odorant to enter the atmosphere through sniffing, which is the behavior a canine uses to smell. Dogs typically sniff 4-7 times per second while in odor, that is, while searching for 
an olfactory cue $e^{91,92}$, though they can sniff up to about 20 times per second in hunting-type scenarios ${ }^{93}$. The outward puff of air produced by a sniff disturbs the ground, which raises odorants and dust. The inward pull of air then facilitates the entrance of odorants into the nostrils. It also can humidify and warm odorants, which aids adsorption to the mucosa ${ }^{87,91}$.

In addition to the physical components and function of the nose, and the chemical properties of the odorant itself, it is important to understand the physical movement and transport of odorants in the atmosphere. The dispersion of the odor is known as an odor plume. Many factors affect an odor plume, including temperature, humidity, wind or other forms of air resistance, and present physical structures, such as trees. Temperature and humidity will affect the volatility of a given molecule, determining both whether it enters the atmosphere and how far it will rise. Wind and air movement have a large effect on odor plumes. Small eddies within a plume redistribute odorants within the plume, while larger eddies redistribute odorants in space, affecting odorant concentration ${ }^{94}$. Whenever the airflow contacts a solid surface, such a tree, a boundary is formed that will further affect molecule dispersion. Additionally, the spatial and temporal scales are behaviorally relevant factors that affect a dog's perception of the odor plume. The spatial scale refers to the area which the canine's nose samples, the search pattern of a canine, and the location of the odor plume itself. The temporal scale refers to the sniffing frequency of the canine, the time it takes the olfactory system to recognize the sniffing event, and the olfactory memory of the canine ${ }^{94}$. 


\subsubsection{Canine Training and Training Aids}

Broadly stated, canine training occurs in a two-step process, classical conditioning and operant conditioning, though these can be taught in either order. Classical conditioning is referred to as imprinting by most canine trainers and is the initial period where a trainer pairs an odor with a reward so that the dog is conditioned to think of the odor itself as reward $^{95}$. The training process involves timing the presentation of the actual reward (known as variable reward) so that the dog reacts to the odor in the same manner each time it is found, regardless of when or how often the reward is received. Operant conditioning focuses on training the behavior that the canine displays once the target odor is found and the target identified ${ }^{95}$. The goal of the process is to create an accurate and reliable canine that is capable of independence, which is very important since environmental detection canines search large land or water areas containing a high number of distractors ${ }^{83}$.

Canines that become very familiar with their odor may begin to generalize or discriminate. Generalization is when a small difference between like odors is ignored. Conversely, discrimination occurs when those small differences are accentuated ${ }^{95}$. For example, canines searching for infected avocado trees should generalize between all species of avocado tree, while discriminating between infected and healthy trees. The processes of generalizing and discriminating can be trained or controlled using the classical and operant conditioning methods of training. Balancing the ability to generalize versus discriminate is especially important in environmental detection canines, where many similar odors are present since canines work in natural situations with many flora and fauna that could potentially distract from or mask the target odor. 
While canines have been used for the environmental detection of various targets, application in the ecological or agricultural arenas is still not widespread, because of the lack of affordable and reliable mimic training aids that provide safe, long-lasting, and easily accessible alternatives ${ }^{96}$. For many environmental targets, nonliving training aids can be found, such as scat, nests, burrows, carcasses, pheromones, or other items left behind by the target species ${ }^{96,97}$. However, for many targets in wildlife detection, nonliving training aids cannot be utilized, and live training aids present high risk searches ${ }^{96,97}$. Also, there is an adjustment period for canines in training to teach them to generalize between their training aid and their naturally occurring target ${ }^{96}$. For plants or pests such as fungi, living training aids are often not possible because of the high risk for spreading the targeted species. Additionally, training a canine using a live target presents several challenges regarding rarity of the species, legality of obtaining and maintaining the species, methods of acquisition, and possibilities of spreading the species should it escape the containment system. Alternative or mimic training aids can be effective in preventing the use of live species as training aids. Though mimic training aids have not yet been applied to environmental substance detection, canines are nonetheless being trained in this field. The training process typically includes using hazardous or dangerous live substances and is unique to each individual target species and canine trainer, leading to a variety of approaches. Alternative or mimic training aids can enhance training by making this process more uniform across the field.

Alternative training aids provide a method of scent delivery for the target compound that does not have any of the drawbacks associated with the actual material ${ }^{98,99}$. The target material is used to create this nonhazardous aid by incorporating the odor in 
another delivery method, such as silica or cotton. Mimic training aids, on the other hand, do not use the target material at all to produce the odor. Mimics produce a synthetic or natural chemical compound(s) to replicate the target's headspace ${ }^{99}$. To create a valid mimic training aid, the active odors in the headspace of the target species must be identified. Active odors are determined by studying the chemical abundance of the volatile organic compounds (VOCs), the physiological sensitivity of the canine to the respective chemicals, and how the canine is trained ${ }^{100}$. Previous reviews of active odor identification for various target odors exist elsewhere ${ }^{101-104}$. The most common process of developing mimics involves laboratory analysis using headspace solid phase microextraction-gas chromatography-mass spectrometry (HS-SPME-GC-MS) followed by extensive and often time-consuming trial-by-error canine field experiments ${ }^{101-104}$. Alternative and mimic training aids preclude the possibility of accidentally spreading the live species and minimize issues of legality, acquisition, movement, and containment.

One type of alternative or mimic training aid used is called a controlled odor mimic permeation system (COMPS) ${ }^{105}$ (Figure 7). The COMPS supply a deployable training aid containing known amounts of active odorant(s) within a vehicle such as cellulose, providing reproducible and known dissipation rates. It is a simple, disposable, and low cost training aid assembled from permeable polymer containers stored inside non-permeable packaging, which allows pre-equilibration. The design is advantageous because they can be optimized for any desired target odor and are not prone to contamination from outside sources ${ }^{105}$. The detection canines trained on the laurel wilt odor were trained utilizing COMPS containing infected wood. 


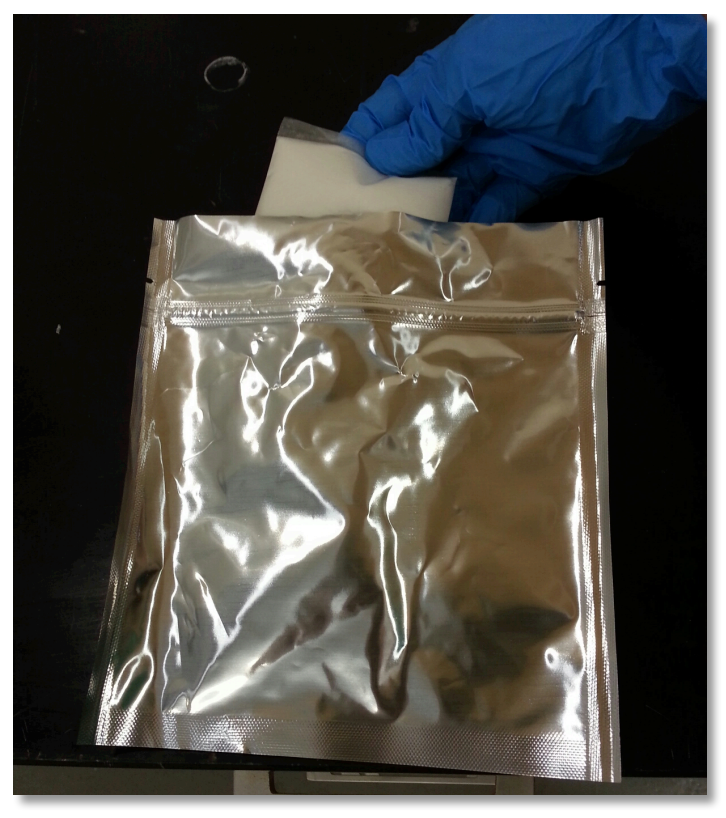

Figure 7: Example of COMPS.

The current study aims to address the difficulties associated with utilizing a live target by evaluating current training aids and then creating an alternative low-risk training aid. The method presented rapidly identifies the active odors of target species, thereby decreasing the time it takes to create low-risk mimic training aids. The present study focuses on the invasive fungus Raffaelea lauricola as a proof of concept.

\subsection{Instrumental Analysis}

Canine detection is typically referred to as a "black-box technology" since many of the compounds and target odors detected by canines are unknown ${ }^{104}$. However, significant progress has been made in identifying such VOCs using gas chromatography-mass spectrometry (GC-MS). The present research utilized headspace solid phase microextraction-gas chromatography-mass spectrometry (HS-SPME-GC-MS). Headspace 
was collected in one of two ways. Either the sample was enclosed in an air-tight container to allow equilibrium of the headspace to be reached, or headspace from around the sample is collected in vapor sample bags prior to SPME fiber exposure. Headspace SPME is a method of extracting analytes from the headspace of a sample. Then, GC-MS is used to separate and analyze the extracted compounds.

\subsubsection{Collection and Extraction Methods}

Solid phase microextraction is a common method for vapor or volatile sampling and is useful for identifying the presence and relative abundance of analytes in the headspace of solid or liquid samples ${ }^{124}$. Solid phase microextraction utilizes a fused silica fiber coated with an ab/adsorbent polymer. Solid phase microextraction is solvent free, so it is suitable for direct extraction of gas-phase analyte vapors from headspace. The amount of analyte vapor extracted depends on the vapor concentration of analytes in the headspace and the partitioning equilibrium of those analytes into the SPME fiber coating. The threephase system can be defined by the following equation:

$$
n_{f}=\frac{K_{f s} V_{f} V_{s} C_{0}}{K_{f s} V_{f}+K_{h s} V_{h}+V_{s}} \quad \text { (Equation 1) }
$$

Where $K_{f s}$ is the partition coefficient of the analyte between the fiber coating and the aqueous phase, $V_{f}$ is the volume of the fiber coating, $V_{s}$ is the volume of the sample, $C_{0}$ is the initial analyte concentration, $K_{h s}$ is the partition coefficient of the analyte between the headspace and aqueous phases, and $V_{h}$ is the volume of the headspace ${ }^{124}$. Notably, SPME is an equilibrium technique, so not all analytes are extracted from the matrix. Additionally, 
the amount of analyte extracted and the detection limits are independent of the location of the fiber in the headspace.

There are several available fiber coatings, which can optimize target analyte extraction. Each fiber coatings will have varying extraction efficiencies for different target analytes and volatility ranges (Table 3). Coatings are generally categorized as nonpolar, polar, or bipolar. Nonpolar coatings retain hydrocarbons; and polar coatings retain polar compounds. Coatings should be tested and optimized for each target analyte or sample.

Table 3: Examples of commercially available SPME fibers.

\begin{tabular}{|l|l|l|l|}
\hline & Acronym & Fiber Chemistry & Polarity \\
\hline 1 & PA & Polyacrylate & Polar \\
\hline 2 & PEG & Polyethylene glycol & Polar \\
\hline 3 & DVB/CAR & Divinylbenzene/Carboxen & Bi-polar \\
\hline 4 & CAR/PDMS & Carboxen/Polydimethylsiloxane & Bi-polar \\
\hline 5 & DVB/CAR/PDMS & Divinylbenzene/Carboxen/Polydimethylsiloxane & Bi-polar \\
\hline 6 & PDMS & Polydimethylsiloxane & Nonpolar \\
\hline
\end{tabular}

The fiber is kept inside a protective sheath (Figure 8). To expose the fiber to the target analytes above a solid sample, the sheath pierces a septum in the vial containing the sample, and a plunger is lowered, exposing the fiber to the headspace. Once a fiber has been exposed to a headspace sample for a predetermined time, it is then injected directly 
into a GC injection port for desorption. With increased temperature in the injection port, the coating/gas partition coefficients decreases. The heat plus the constant flow of carrier gas decreases the fiber coating's ability to retain analytes ${ }^{124}$.

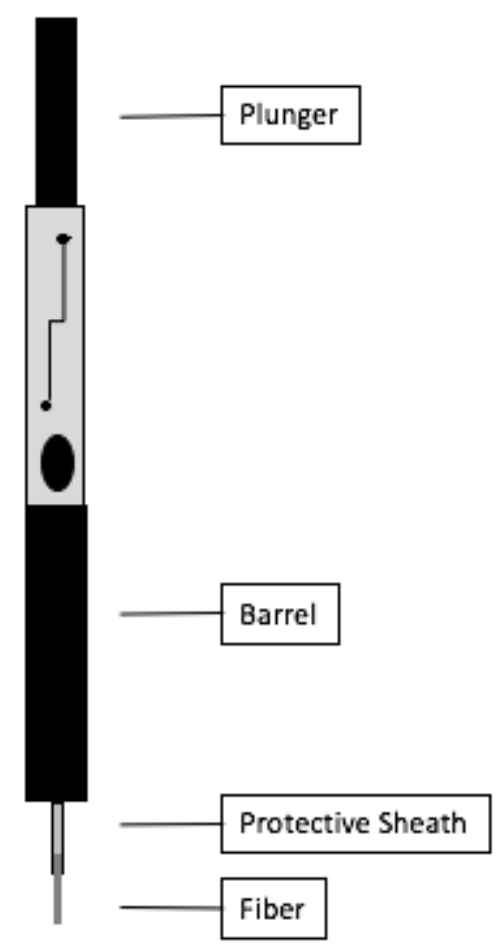

Figure 8: SPME fiber diagram.

Solid phase microextraction is generally considered a semi-quantitative technique of analysis. However, using an internal standard can increase analytical ability. An internal standard added directly to a sample is not ideal because the standard would alter all partition coefficients for the analyte. Using an external standard decreases the number of altered variables, thereby increasing reliability. The method, called externally sampled internal standard (ESIS) $)^{125,126}$ (Figure 9), uses Equation 2 for analysis: 


$$
\frac{\boldsymbol{A}}{E}=\frac{\text { peak area of analyte }}{\text { peak area of ESIS }} \quad(\text { Equation 2) })^{125,126}
$$

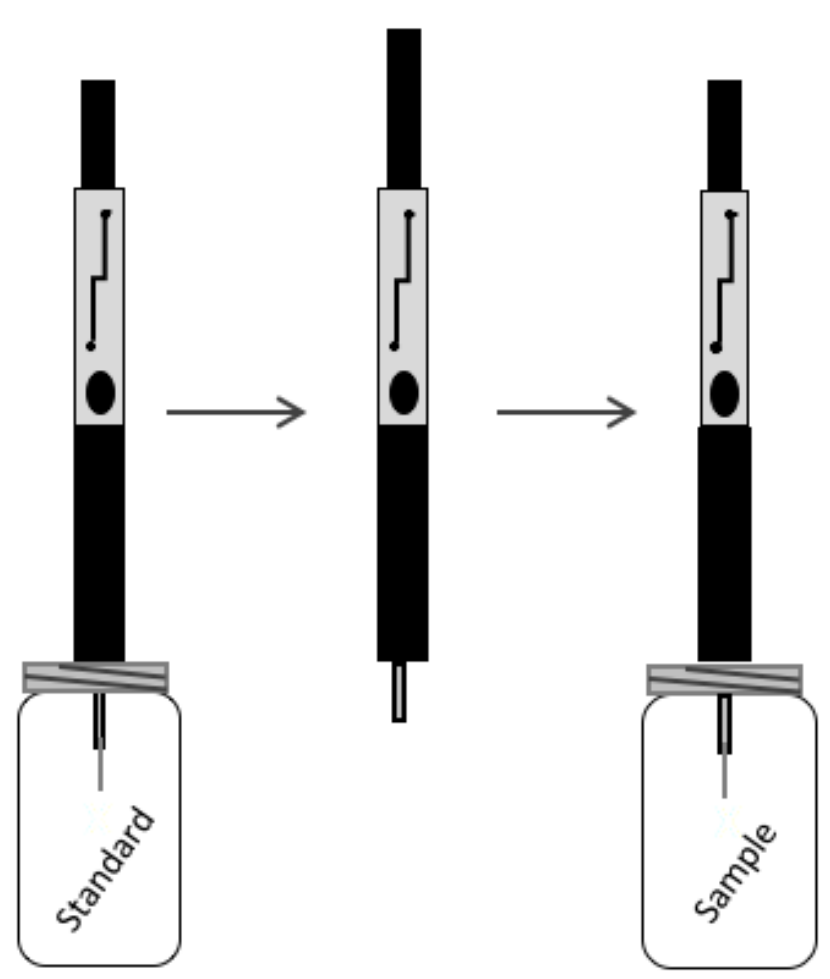

Figure 9: Basic SPME-ESIS diagram.

Though SPME and ESIS-SPME are advantageous when extracting from simple matrices, when extracting the headspace of a complex matrix, there may be many compounds with varying volatilities that limit an accurate quantification of compounds as a consequence of fiber capacity. Vapor sample bags are a whole-air sampling method that can help resolve the disadvantage of low fiber capacity. Air is collected using a vacuum sample pump connected to an inlet in the bag (Figure 10). The sample bags are field deployable and can be used to collect and store samples of air for later extraction and analysis. Field collection is especially advantageous when a sample cannot be placed in an 
enclosed container where headspace equilibrium cannot be established. Sample bags are made in a variety of polymers, so that the optimal type can be chosen for the collection and storage of target analytes. Parameters affecting the suitability of a polymer bag include permeation through the bag walls, adsorption to the bag, and chemical interference from the bag. Despite these phenomena, vapor sample bags are easy to use, reusable, and durable ${ }^{127}$. Certain vapor sample bags come with septa so that SPME fibers can be introduced once a sample is collected.

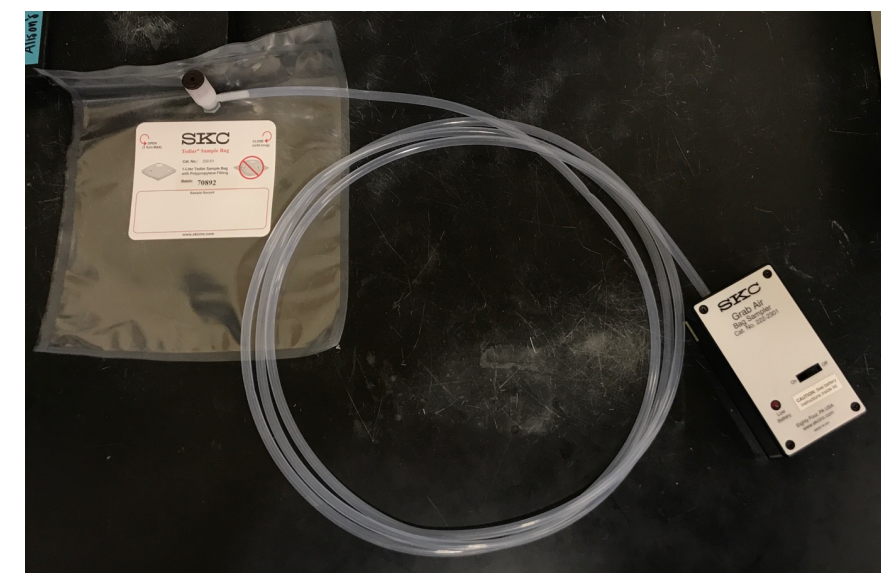

Figure 10: Vapor pressure bag with connected handheld vacuum pump.

\subsubsection{Gas Chromatography-Mass Spectrometry}

Gas chromatography-mass spectrometry is a method of separation, ionization, and identification for chemical compounds (Figure 11). Chromatography separates components of a gaseous mixture by passing the mixture through a column containing two immiscible phases, the stationary and mobile phases. The stationary phase is immobilized on the column while the mobile phase, a carrier gas such as nitrogen or helium, carries the 
mixture. Like SPME fibers, there are several different commercially available chemistries of column. The column is stored in a temperature controlled oven at atmospheric pressure.

The SPME fibers can be introduced directly into a GC injector. A split/splitless injector was used in the present study. The SPME fiber is exposed into a heated glass liner, desorbing the volatile compounds from the fiber, and the carrier gas flows past the fiber, bringing the compounds into the column. In split mode, the carrier gas flows through a vent valve that separates the flow into two fractions, only one of which is introduced to the column. In splitless mode, the entire mixture is introduced. Generally, splitless mode is used for quantitation and trace analysis ${ }^{128-130}$.

Once in the column, the components of the mixture will interact with surface of the stationary phase and elute at individual times. Gas chromatography is thus a measure of migration times of different chemical species in relation to the column. These migration times, are known as retention times. A specified compound will have a consistent retention time for a set of given parameters, such as temperature ramp, column width, column length, column chemistry, and carrier gas flow, each of which should be optimized. Each compound's retention time is recorded on a chromatogram, where each compound is recorded as a separate Gaussian-shaped peak ${ }^{128-130}$. The partition coefficient defining this action for a given compound is described by:

$$
K_{D}=\frac{C_{L}}{C_{G}} \quad \text { (Equation 3) }
$$

where $K_{D}$ is the distribution coefficient of the compound, $C_{L}$ is the concentration of the compound in the stationary phase, and $C_{G}$ is the concentration of the compound in the mobile phase ${ }^{104}$. 
Retention, separation, and resolution factors can each affect the exact shape of these peaks and ultimately define the column's efficiency. The retention factor, $k$, is the ratio of the mass of an introduced compound that is retained in the mobile phase to the mass of that compound retained in the stationary phase ${ }^{130}$. Retention factor describes the capacity of the column and the ability of the column to retain each compound. The separation factor, $\alpha$, allows the comparison of adjacent peaks in the same chromatogram ${ }^{130}$. It is the ratio of either the two retention times minus the dead time (the time is takes the carrier gas to reach the detector) or the two retention factors. Separation factor parameter is used to describe whether separation of compounds is possible or has been achieved. The final factor is the resolution factor, $R$, which quantifies the separation between two chemical species ${ }^{130}$. Unlike the other factors, $R$ is not a ratio. Rather, it considers both the widths of the peaks and the retention times of each compound. When considered together, the retention, separation, and resolution factors are used to define the usefulness of a specified chromatographic method in chemical mixture separations.

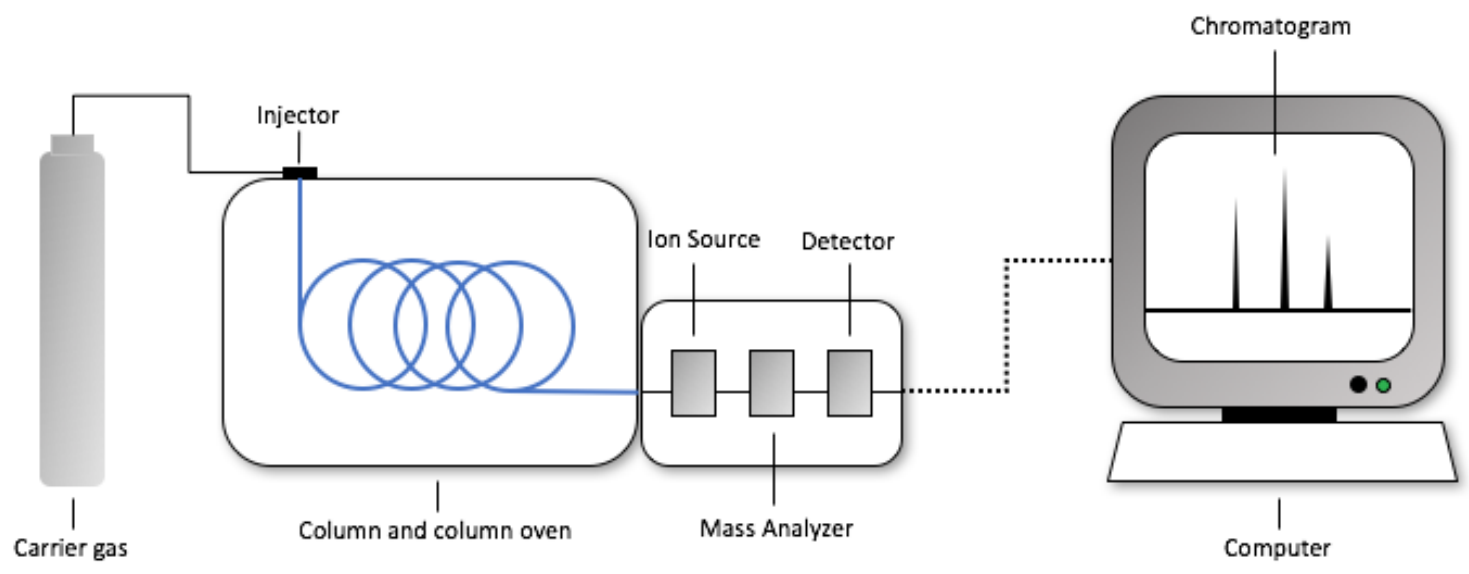

Figure 11: Basic GC-MS diagram. 
After eluting from the column, the compounds enter the mass spectrometer for detection and analysis. Mass spectrometry is utilized because it is extremely sensitive and has low limits of detection. Compounds exiting the GC first enter the mass analyzer and are ionized through a fragmentation process. These ions are then separated by their massto-charge ratios $(\mathrm{m} / \mathrm{z})$ and are then detected in proportion to their abundance. Since each compound fragments in a unique pattern, each structure will have a unique mass spectrum, which is the recording of its $\mathrm{m} / \mathrm{z}$ and the ion abundances. The ionization process is destructive, but utilizes only a small amount of sample ${ }^{130,131}$.

The current study used a mass spectrometer with an electron ionization ion source with either an ion trap mass analyzer or quadrupole mass analyzer. Electron ionization (EI) is often used for gas phase molecules, and causes high amounts of fragmentation that can be used for molecular identification. In EI, a heated filament produces electrons that are accelerated towards an anode. The electron beam crosses the stream of gaseous molecules from the GC, and transfers energy to the molecules, exciting the molecules and causing fragmentation. Fragmentation yields positive ions that then enter the mass analyzer. An ion trap mass analyzer uses oscillating electric fields to separate ions. Essentially, ions are contained in the trap by alternating potentials, which repels the ions each time the potential is switched. In this process, the ions are also accelerated on the basis of their $\mathrm{m} / \mathrm{z}$ so that different ions are at different locations in the trap. Ions can be ejected from the trap using an applied voltage and propelled toward the detector, which uses sensors to measure electrical change and converts the signals into a mass spectrum ${ }^{130,131}$. A quadrupole mass analyzer separates ions based on the same principles as an ion trap, except that quadrupoles 
consist of four parallel rods of alternating potentials that the ions travel through, rather than a $\operatorname{trap}^{131}$.

The efficiency of a mass spectrometer can generally be described by several methods. First, the mean free path, $L$, describes the distance an ion can travel without unwanted reactions that could increase the complexity, and thereby decrease efficiency or specificity, of the spectrum. The path can be given by:

$$
L=\frac{k T}{\sqrt{2} p \sigma} \quad(\text { Equation } 4)^{131}
$$

where $k$ is the Boltzmann constant; $T$ is the temperature in $\mathrm{K} ; p$ is the pressure in $\mathrm{Pa}$; and $\sigma$ is the collision cross-section $\left(\mathrm{m}^{2}\right)^{131}$. Second, the upper and lower mass limits define the range of $\mathrm{m} / \mathrm{z}$ values detectable by a certain instrument. Also, sensitivity of the instrument can be defined using the weight of the sample consumed per second ${ }^{131}$.

\subsubsection{Scanning Electron Microscopy}

Scanning electron microscopy (SEM) is a method of generating high resolution, high magnification images under vacuum. It uses a beam of highly energetic electrons to interact with the surface of the sample and produce the image ${ }^{130}$. The electron beam produces a secondary stream of electrons from the sample's surface, which are then extracted into an acceleration tube and detected by signal detectors, producing the desired image ${ }^{132}$. The image provides information about the sample's topography and chemical composition.

Because an SEM must function under high vacuum, biological samples must go through a process to freeze and dry the cells, preventing bursting under vacuum, which would otherwise distort the sample's image. Additionally, biological samples are non- 
conductive, and must therefore be coated with carbon or a metal using a sputtercoater prior to being placed in the SEM. Wood specimen should be first be cut carefully using fresh steel blades, preferably while wet to provide the cleanest cut ${ }^{133}$, and then fixed by using a fixative, such as glutaraldehyde, to quickly kill, sterilize, and stabilize cells. Next, the specimen are dried or dehydrated, mounted on a stub, and coated with a conductive material, such as gold or palladium ${ }^{133,134}$.

\subsection{Statistical Analysis}

To best understand and apply the data obtained through HS-SPME-GC-MS, several different methods of statistical analysis were utilized. They are each discussed in detail in the following sections.

\subsubsection{Principal Components Analysis}

Principle components analysis (PCA) is a method of statistical analysis used to reduce large amounts of data when there is a correlation present. It does not assume the data have a particular distribution, and is therefore free from that bias. The PCA identifies principle components of the data, which are new linear combinations of the given variables describing each piece of information. These principle components explain the variation in the data set. For example, if the data set is $X_{1}, X_{2}, \ldots X_{n}$, then the principle components are

$Z_{1}, Z_{2}, \ldots Z_{n}$. The first principle component (PC1), which is also the most informative, would then be $Z_{1}=a_{11} X_{1}+a_{12} X_{2} \quad \ldots a \operatorname{alnXn}{ }^{135}$. Each principle component corresponds to an eigenvalue, where PC1 has the largest eigenvalue, and each subsequent principle component has a decreasing eigenvalue. Eigenvalues give the amount of variance 
explained by its corresponding principle component. Using the principle components as coordinate axes, the data can then be plotted in a new coordinate system that further visualizes the relationships or correlations ${ }^{135,136}$.

\subsubsection{Cloud Plots}

The XCMS Online program was used to produce cloud plots ${ }^{137}$. The program utilizes raw chromatographic data files to first correct retention time deviation and then analyze significant ion intensity differences through pairwise statistical analysis. A cloud plot showed both fold change in ion intensity and level of significance for these changes. Significant ion intensities in detected metabolite features are shown overlaid on aligned chromatograms and plotted on the basis of retention time (minutes) on the $\mathrm{x}$-axis and $\mathrm{m} / \mathrm{z}$ on the y-axis. The p-value and fold change can be adjusted for desired values for each detected metabolic feature. For the present studies, $p$-value $\leq 0.01$ and fold change $\geq 1.5$. Toggle upregulated and downregulated features are differentiated on the basis of color, where green demonstrates toggle upregulation, or fold increases, and red demonstrates toggle downregulation, or fold decreases. The intensity of the color of each circle indicates the statistical significance of each feature difference, so that low p-values are represented by darker colors. The radii of the circles indicate the log-fold increase or decrease in abundance, where larger circles indicate larger fold changes ${ }^{137}$.

\subsubsection{Box-and-Whisker Plots}

The XCMS Online program can also produce box-and-whisker plots for each detected metabolic feature ${ }^{137}$ (Figure 12). A box-and-whisker plot is a simple form of data 
representation showing data distribution over a line. Each end of the line marks the range of the data. The median is also marked. A box appears on the line showing the upper and lower quartiles of the data as they relate to the lowest, highest, and median values ${ }^{135}$.

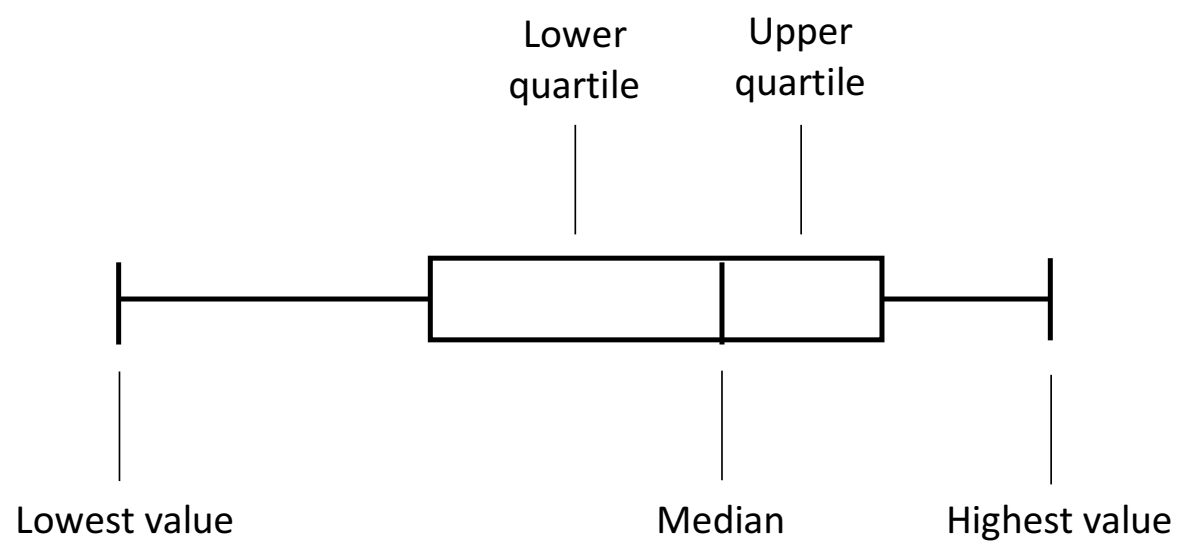

Figure 12: Example of a box-and-whisker plot.

\subsubsection{Predictive Values}

There are four possible outcomes of any given canine search: true positive, false positive, false negative, and true negative (Table 4). A true positive is when the target is present and the canine correctly indicates it. A false positive is when the target is not present, but the canine indicates that it is. Conversely, false negative is when the target is present, but the canine indicates it is not or has no reaction. Finally, a true negative is when the target is absent, and the canine correctly indicates this or has no response. Each of these values is important to consider when determining the efficiency of a canine or assessing its performance. Predictive values are therefore used to define canine accuracy in field trial performance ${ }^{138}$. 
Table 4: Possible canine decision or search outcomes.

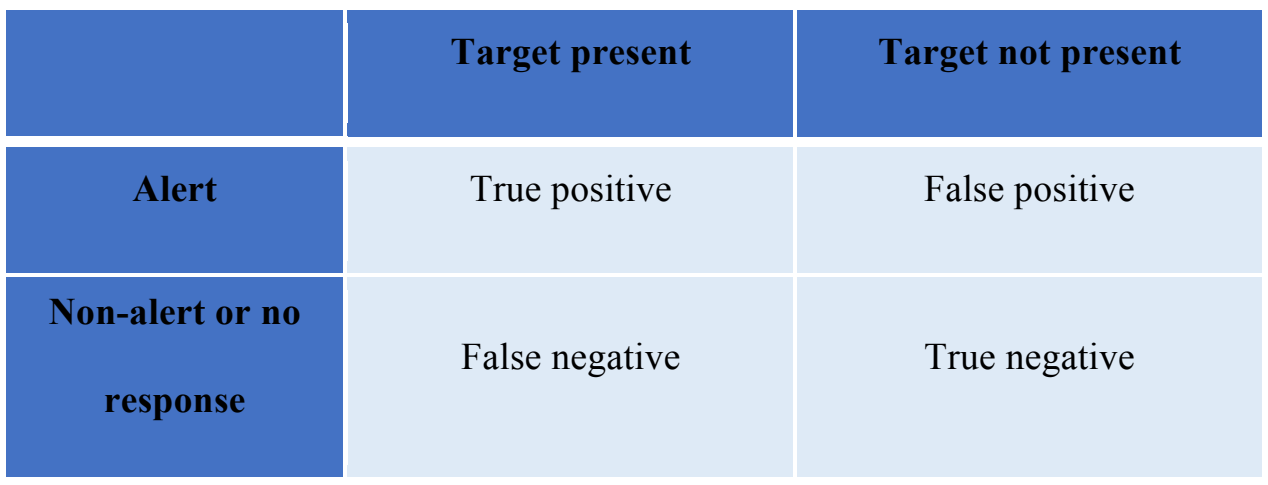

The first predictive value is known as the positive predictive value PPV, and is given by:

$$
P P V=\frac{\text { True positive }}{\text { True positive }+ \text { False positive }} \quad \text { (Equation 5) }
$$

PPV describes the probability that a positive answer, or canine alert, is correct. Contrarily, the negative predictive value (NPV) describes the probability that a negative answer, or canine non-alert, is correct. It is given by:

$$
N P V=\frac{\text { True negative }}{\text { True negative }+ \text { False negative }} \quad \text { (Equation 6) }
$$

Together, these predictive values are used to measure the likelihood of a target's presence given a specific canine's response to the situation. It is important to understand both aspects because, for example, a canine that has a high true positive rate, yet a high false positive rate, will have a low predictive value. The bias of this canine to alert makes it less reliable ${ }^{138,139}$.

\section{SIGNIFICANCE OF STUDY AND RESEARCH OBJECTIVES}

Use of detection canines for environmental and agricultural applications is limited by a lack of research to resolve issues such as the rarity of the species, legality of obtaining 
and maintaining the species, methods of acquisition, and possibilities of spreading the species should it escape the containment system. The current research explores R. lauricola as a proof-of-concept to demonstrate that increased knowledge of a target's odorants can improve the safety and efficacy of training and detection of live targets. Odors of the fungus, infected avocado trees, and healthy avocado trees were each identified. Then, current canine training aids were evaluated. Finally, a novel method of odor separation, collection, and delivery was developed. The results will assist in curtailing the spread of the laurel wilt disease by improving the use of canines as a source of standoff detection and early identification. Moreover, the mechanism for COMPS evaluation and column vent methods devised in the present study will be suited for other novel pests, diseases, and pathogens. The dissertation presents the results of fieldwork and laboratory analyses performed to satisfy these goals. The tasks completed during the research project are outlined below:

1. Optimization of a method for the detection of the VOCs released from the laurel wilt disease
a. SPME fiber selection
b. Extraction time study
c. GC-MS optimization

2. Analytical studies of the laurel wilt disease
a. Examining VOCs of R. lauricola over time
b. Establishing VOCs of infected avocado trees
c. Comparing VOCs from infected avocado trees to those of healthy avocado trees


3. Assessment of a COMPS for the detection of the laurel wilt disease by canines

a. Development of the column vent method for active odor identification

b. Characterization of the canine training aid used in the field

4. Evaluation of the VOCs of the laurel wilt disease in avocados using greenhouse experiments

a. Examining the VOCs of avocado trees inoculated with Raffaelea lauricola

b. Examining the VOCs of treated infected avocado trees

\section{MATERIALS AND METHODOLOGY}

The materials and methodology for each task performed in the dissertation are specific to the needs and design of each experiment. Comprehensive descriptions for each experimental design are presented in this section.

4.1. Task 1: Optimization of a Method for the Detection of the Volatile Organic Compounds Released from the Laurel Wilt Disease

\subsubsection{Solid Phase Microextraction Fiber Selection}

The SPME fibers are available in several different chemistries, as shown in Table 3. Four fiber coatings were tested to optimize the number of compounds extracted, the abundance of compounds extracted, and the reproducibility between extractions: $60 \mu \mathrm{m}$ polyethylene glycol (PEG) for polar compounds, 50/30 $\mu \mathrm{m}$ divinylbenzene/ carboxen/polydimethylsiloxane (DVB/CAR/PDMS) for intermediate polarity compounds, and $100 \mu \mathrm{m}$ polydimethylsiloxane (PDMS) and $65 \mu \mathrm{m}$ polydimethylsiloxane/ divinylbenzene (PDMS/DVB) for non-polar compounds. All fibers were purchased from 
Supelco (Bellefonte, PA). Seven compounds were tested in a 100ppm mixture in methylene chloride: 1-methoxy-2-propanol, heptanal, dodecane, benzaldehyde, heptyl ether, 2phenethyl acetate, and 2-phenylethanol. All compounds were purchased from SigmaAldrich (St. Louis, MO), with the exception of 1-methoxy-2-propanol, which was purchased from Fluka (Milwaukee, WI). These compounds were chosen as examples of common VOCs obtained from molds and/or fungi ${ }^{12,140}$. In a $40 \mathrm{~mL}$ glass vial with PTFE/silicone septa screw caps (Supelco, Bellefonte, PA), $5 \mu \mathrm{L}$ of the $100 \mathrm{ppm}$ mixture was allowed to equilibrate for thirty minutes. Then, a fiber was exposed for a thirty minute extraction. Each trial was performed in triplicate, plus an extraction and instrument blank.

Analytes were desorbed for 20 minutes at $250^{\circ} \mathrm{C}$ directly from fibers into a Varian 3800 Gas Chromatograph/Saturn 2000 Ion Trap Mass Spectrometer (Walnut Creek, CA) injection port (1:1 split ratio) and separated over a $0.25 \mathrm{~mm}$ ID solgel-wax column (SGE Analytical Science, Pflugerville, TX). Helium was used as the carrier gas at $1 \mathrm{~mL} / \mathrm{min}$. The temperature gradient was $10^{\circ} \mathrm{C}$ per minute from $50^{\circ} \mathrm{C}$ to $120^{\circ} \mathrm{C}$ and $11^{\circ} \mathrm{C}$ per minute from $120^{\circ} \mathrm{C}$ to $260^{\circ} \mathrm{C}$. A solvent delay was used from 2.5 to 6 minutes. Compounds were identified using linear retention time and confirmed using standard mixtures.

\subsubsection{Extraction Time Study}

Once a fiber coating was chosen as the optimal chemistry for target analytes, it was tested for ideal extraction time. The 50/30 $\mu \mathrm{m}$ DVB/CAR/ PDMS fiber coating was exposed to $5 \mu \mathrm{L}$ of the same 100ppm mixture described above for each of the following times: $0.5,1,5,10$, and 20 hours. Each trial was performed in triplicate, plus an extraction and instrument blank. Samples were analyzed by the method described in Section 4.1.1. 


\subsubsection{Gas Chromatography-Mass Spectrometry Optimization}

For tasks 1 and 2, a Varian 3800 Gas Chromatograph/Saturn 2000 Ion Trap Mass Spectrometer (Walnut Creek, CA) with a $0.25 \mathrm{~mm}$ ID solgel-wax column (SGE Analytical Science, Pflugerville, TX) was used. For tasks 3 and 4, an Agilent Technologies HP 6890 Series Gas Chromatography System/HP 5973 Mass Selective Detector (Santa Clara, CA) with a $0.25 \mathrm{~mm}$ ID solgel-wax column (SGE Analytical Science, Pflugerville, TX) was used. Both were optimized for the following parameters: temperature ramp, solvent delay, desorption temperature, and desorption time. Optimization was performed using the abovementioned 100ppm mixture and DVB/CAR/PDMS SPME fibers.

\subsection{Task 2: Analytical Studies of the Laurel Wilt Disease}

\subsubsection{Examining Volatile Organic Compounds of Raffaelea lauricola Over Time}

\subsubsection{Fungal Cultures}

Fungal cultures of $R$. lauricola (TREC, Homestead, FL) were stored on potato dextrose agar (PDA) slants at room temperature $\left(21^{\circ} \mathrm{C}\right)$. A total of 78 cultures were prepared in $20 \mathrm{~mL}$ clear glass vials with PTFE/silicone septa screw caps (Supelco, Bellefonte, PA) in preparation for SPME. In the same manner, 26 vials containing only PDA were prepared as controls. The caps were placed on top of the vials and secured with Parafilm (Bemis, Neenah, WI) to continue to allow continued oxygen access. All chemicals used in standards were obtained from Sigma-Aldrich (St. Louis, MO). 


\subsubsection{Identification of Volatile Organic Compounds}

The study was performed over a 28 day period. For all samples, agar was streaked with the fungus one week prior to the beginning of the study. Each day of the observation period, three vials of $R$. lauricola cultures were analyzed, and the results combined to obtain a daily average. Prior to performing SPME, the Parafilm was removed from each vial and each lid was tightened to avoid loss of VOCs, and the samples were left for a one hour equilibration period. The DVB/CAR/PDMS SPME fibers were exposed to the headspace above the cultures for one hour VOC extraction. Analytes were then desorbed for 20 minutes at $250^{\circ} \mathrm{C}$ directly from fibers into a Varian $3800 \mathrm{Gas}$ Chromatograph/Saturn 2000 Ion Trap Mass Spectrometer injection port (10:1 split ratio) and separated over a $0.25 \mathrm{~mm}$ ID solgel-wax column. Helium was used as the carrier gas at $1 \mathrm{~mL} / \mathrm{min}$. The temperature gradient was $10^{\circ} \mathrm{C}$ per minute from $50^{\circ} \mathrm{C}$ to $120^{\circ} \mathrm{C}$ and $11^{\circ} \mathrm{C}$ per minute from $120^{\circ} \mathrm{C}$ to $260^{\circ} \mathrm{C}$. Compounds were identified using linear retention time and confirmed using standard mixtures when possible, or a mass spectral library (NIST MS Search 2.0 and AMDIS, Gaithersburg, MD) when authentic standards were unavailable. All chemical compounds were analytical grade and were obtained from Sigma-Aldrich (St. Louis, MO). Analyses of the resulting data were performed semi-quantitatively. 
4.2.2. Establishing Volatile Organic Compounds of Infected Avocado Trees and Comparing Volatile Organic Compounds from Infected Avocado Trees to Those of Healthy Avocado Trees

\subsubsection{Sampling Protocol}

Sampling of VOCs from both uninfected and infected avocado trees (Persea americana) took place in commercial avocado groves in South Florida. Results from a total of 15 infected and 9 uninfected trees of the Lula variety are presented herein. Infected trees were selected if there was visible wilting. Uninfected trees were selected if there was no visible wilting, and there was no wilting in the surrounding two rows of trees (Figure 13). The method was chosen following the most current recommendation for trenching to isolate infected trees from uninfected areas ${ }^{141}$. Laurel wilt presence in each grove was confirmed through DNA analysis of $R$. lauricola.

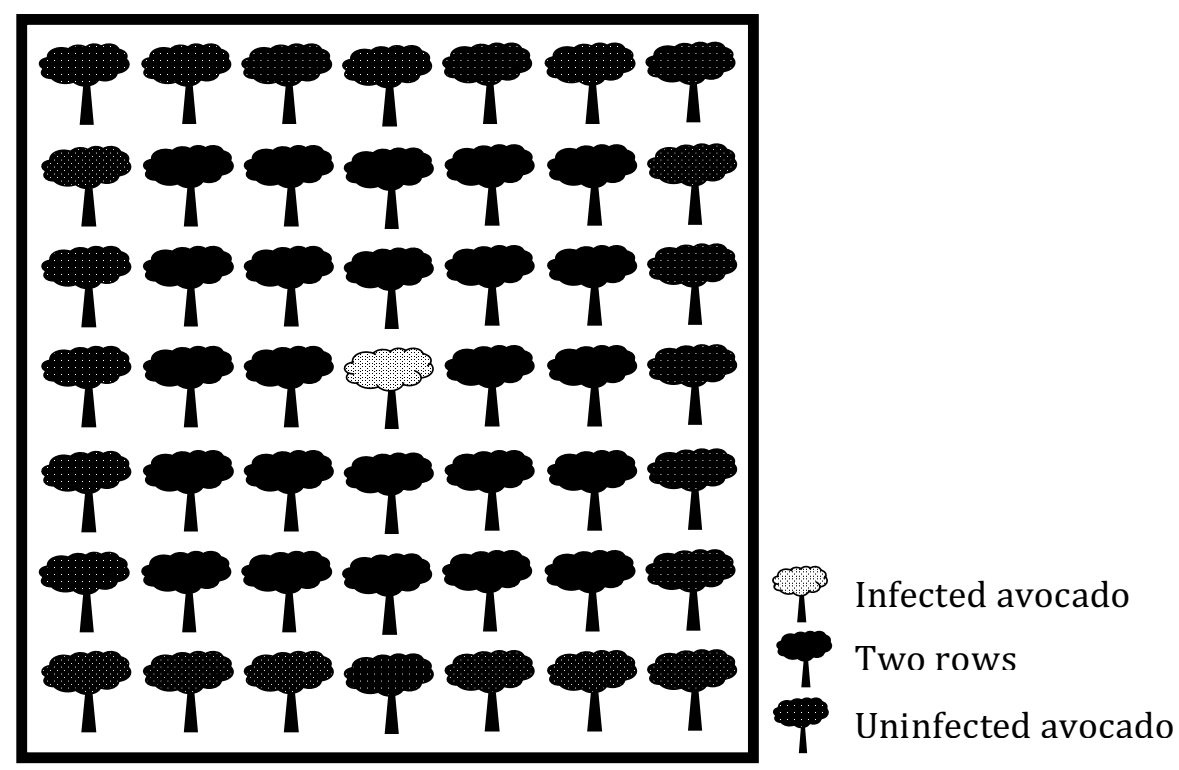

Figure 13: Schematic showing sample selection in avocado groves. There is a two-row barrier of nonsymptomatic trees between infected and uninfected samples. 
Location and relevant weather data were recorded for samples collected by the author, including: infection or no infection, time and day, temperature, humidity, and dew point (Appendix Table 17). Sampling was conducted using a sterilized hatchet. Samples were taken from the trunk of each tree because their volatiles are less variable and their diameters make them the target of $X$. glabratus as well as the host of $R$. lauricola ${ }^{2}$. Samples of the bark, phloem, and wood were collected from the trunks from between four and five feet high and placed together in 16oz. sterilized glass Mason jars for transportation to the laboratory. Within 24 hours, the samples were taken for analysis using laboratory protocols. Caps were tightened by screwing down all the way then turning back once so as to allow oxygen flow and prevent an anaerobic atmosphere.

\subsubsection{Laboratory Protocol}

All tests were performed in triplicate. Subsamples $(3.00 \mathrm{~g})$ containing mixtures of bark, phloem, and xylem were placed in $20 \mathrm{~mL}$ clear glass vials with PTFE/silicone septa screw caps. The cap was placed on top of the vial and wrapped in Parafilm for one hour. Then, the caps were tightened completely for one hour equilibration of the VOCs. Conditioned DVB/CAR/PDMS SPME fibers were exposed to the headspace above samples for one hour extraction. An extraction and instrument blank was run before each set of triplicate samples.

Analytes were desorbed directly from fibers into a Varian 3800 Gas Chromatograph/Saturn 2000 Ion Trap Mass Spectrometer injection port for separation and analysis. All chemical compounds were obtained from Sigma-Aldrich (St. Louis, MO) except benzaldehyde, which was obtained from Fisher Scientific (Waltham, MA). A 
$0.25 \mathrm{~mm}$ ID solgel-wax column was used with the GC-MS parameters presented in Table 5 and Table 6 .

Table 5: The temperature ramp used for the GC-MS analyses in a Varian 3800 Gas Chromatograph/Saturn 2000 Ion Trap Mass Spectrometer and a Bruker Scion 346-Gas Chromatograph.

\begin{tabular}{|r|r|r|r|}
\hline Temperature $\left({ }^{\circ} \mathrm{C}\right)$ & Rate $\left({ }^{\circ} \mathrm{C} / \mathrm{min}\right)$ & Hold $(\mathrm{min})$ & Total $(\mathrm{min})$ \\
\hline 50 & 0.0 & 5.00 & 5.00 \\
\hline 80 & 10.0 & 6.75 & 14.75 \\
\hline 120 & 10.0 & 8.50 & 27.25 \\
\hline 260 & 11.0 & 0.00 & 39.98 \\
\hline
\end{tabular}

Table 6: The parameters for the Varian 3800 Gas Chromatograph/Saturn 2000 Ion Trap Mass Spectrometer containing a $0.25 \mathrm{~mm}$ ID solgel-wax column.

\begin{tabular}{|l|r|}
\hline \multicolumn{2}{|c|}{$\begin{array}{r}\text { Parameters: } \\
\text { solgel wax column }\end{array}$} \\
\hline Split Ratio: & $10: 1$ \\
\hline Desorption: & $20 \mathrm{~min}$ \\
\hline Low Mass: & $33 \mathrm{~m} / \mathrm{z}$ \\
\hline High Mass: & $300 \mathrm{~m} / \mathrm{z}$ \\
\hline Injection Temp: & $250^{\circ} \mathrm{C}$ \\
\hline Flow rate: & $1.0 \mathrm{~mL} / \mathrm{min}$ \\
\hline
\end{tabular}

4.3. Task 3: Assessment of a Controlled Odor Mimic Permeation System for the Detection of the Laurel Wilt Disease by Canines

\subsubsection{Development of the Column Vent Method for Active Odor Identification}

\subsubsection{Laboratory Protocol}

The study achieved separation and collection of the VOCs in the headspace above infected samples of avocado trees using a $0.53 \mathrm{~mm}$ ID solgel-wax column left venting to the atmosphere (Figure 14). Sample collection and SPME methods were followed according 
to the procedures presented in Sections 4.2.2.1 and 4.2.2.2. The VOCs were desorbed directly from fibers into a Bruker Scion 346-Gas Chromatograph injection port for separation (see Table 5 and Table 7 for parameters). Fractions of the odor were collected on U.S.P. Type VII sterile 2"x2", 8ply cotton gauze (Independent Medical Co-op, Inc., Daytona Beach, FL) in $10 \mathrm{~mL}$ clear glass vials with PTFE/silicone septa caps according to Table 8 .

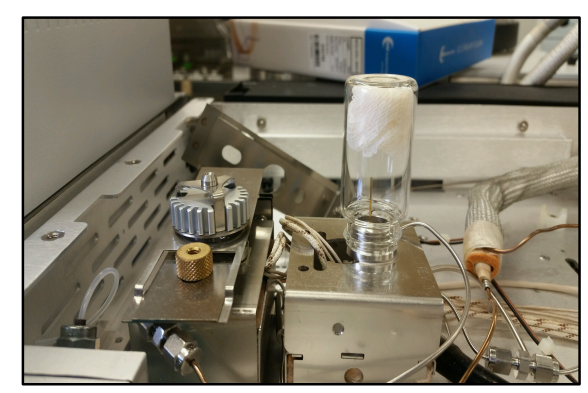

Figure 14: Fraction being collected on cotton gauze in $10 \mathrm{~mL}$ glass vial from $0.53 \mathrm{~mm}$ ID solgel-wax column vented to the atmosphere.

Table 7: The parameters for the Bruker Scion 346-Gas Chromatograph containing a 0.53mm ID solgel-wax column.

\begin{tabular}{|l|r|}
\hline \multicolumn{2}{|c|}{$\begin{array}{c}\text { Parameters: } \\
\text { solgel wax column }\end{array}$} \\
\hline Split Ratio: & Off \\
\hline Desorption: & $20 \mathrm{~min}$ \\
\hline Low Mass: & $33 \mathrm{~m} / \mathrm{z}$ \\
\hline High Mass: & $300 \mathrm{~m} / \mathrm{z}$ \\
\hline Injection Temp: & $250^{\circ} \mathrm{C}$ \\
\hline Flow rate: & $6.0 \mathrm{~mL} / \mathrm{min}$ \\
\hline
\end{tabular}


Table 8: Division of fractions by minute for column vent procedure.

\begin{tabular}{|r|r|r|}
\hline Label & Fraction begin $(\mathrm{min})$ & Fraction end $(\mathrm{min})$ \\
\hline $\mathrm{A}$ & 0.00 & 10.00 \\
\hline $\mathrm{B}$ & 10.01 & 20.00 \\
\hline $\mathrm{C}$ & 20.01 & 30.00 \\
\hline $\mathrm{D}$ & 30.01 & 39.98 \\
\hline $\mathrm{W}$ & 0.00 & 39.98 \\
\hline
\end{tabular}

After a one hour equilibration, SPME fibers were exposed to the headspace of the gauze for a one hour extraction. The fibers were directly desorbed in a Varian 3800 Gas Chromatograph/Saturn 2000 Ion Trap Mass Spectrometer injection port for verification of the VOCs contained in each vial. Note that the only differences between the two GC parameters are the split ratio and flow rate, which had to be altered to ensure the fractions contained accurate separations of the chromatographic data.

\subsubsection{Canine Field Experiments}

Two detection canines trained to detect avocado trees infected with laurel wilt disease were used in the present study. The handler-canine teams were certified through the International Forensic Research Institute/National Forensic Science Technology Center (IFRI/NFSTC) Detector Dog Team Certification utilizing the latest best practices by the Scientific Working Group on Dog and Orthogonal detector Guidelines (SWGDOG) ${ }^{142}$. Canine 1 (Belgian Malinois) and Canine 2 (Dutch Shepherd) were not previously trained for detection work. They were initially trained using scent association with a universal detector calibrant ${ }^{143}$. Canines were then trained for five days per week during two hour sessions. Each dog typically ran two to four trials per training session. Training for laurel wilt disease detection continued for 10 months prior to the study. To verify canine 
accuracy, trials were done at least biweekly in avocado groves containing infected trees. Training was done according to SWGDOG SC2 General Guidelines and the dogs were housed according to SWGDOG SC4 Kenneling and Healthcare ${ }^{142}$. The canines were all trained to sit at the base of a tree containing the target odors as a positive response.

For laurel wilt detection, training aids were made from a combination of bark, phloem, and xylem obtained from infected avocado trees and heat sealed in COMPS ${ }^{105}$ made of 3"x3" 4MIL low density polyethylene (LDPE) bags (Uline Poly Bags, Pleasant Prairie, WI), all stored in the same aluminum bag when not in use. The COMPS were used to supply a deployable training aid containing known amounts of odor, providing reproducible and known dissipation rates.

The fractions (A,B,C,D, and $\mathrm{W}$ as shown in Table 8) collected from the column vent design were presented to the canines in a series of trials. Blank cotton gauze in vials and COMPS containing infected avocado wood were used as controls. Trials were performed in a mango (Mangifera indica) grove and a longan (Dimocarpus longan) grove to avoid possible introduction of avocado tree odors. The vials were randomly selected and then placed 4-5 feet high in trees using a random number generator. Relevant location and weather data were recorded for each trial, including: time and day, temperature, humidity, and dew point. Three trial days were considered for the study for a total of six canine runs. The canine trial designs are given in Figure 15. 


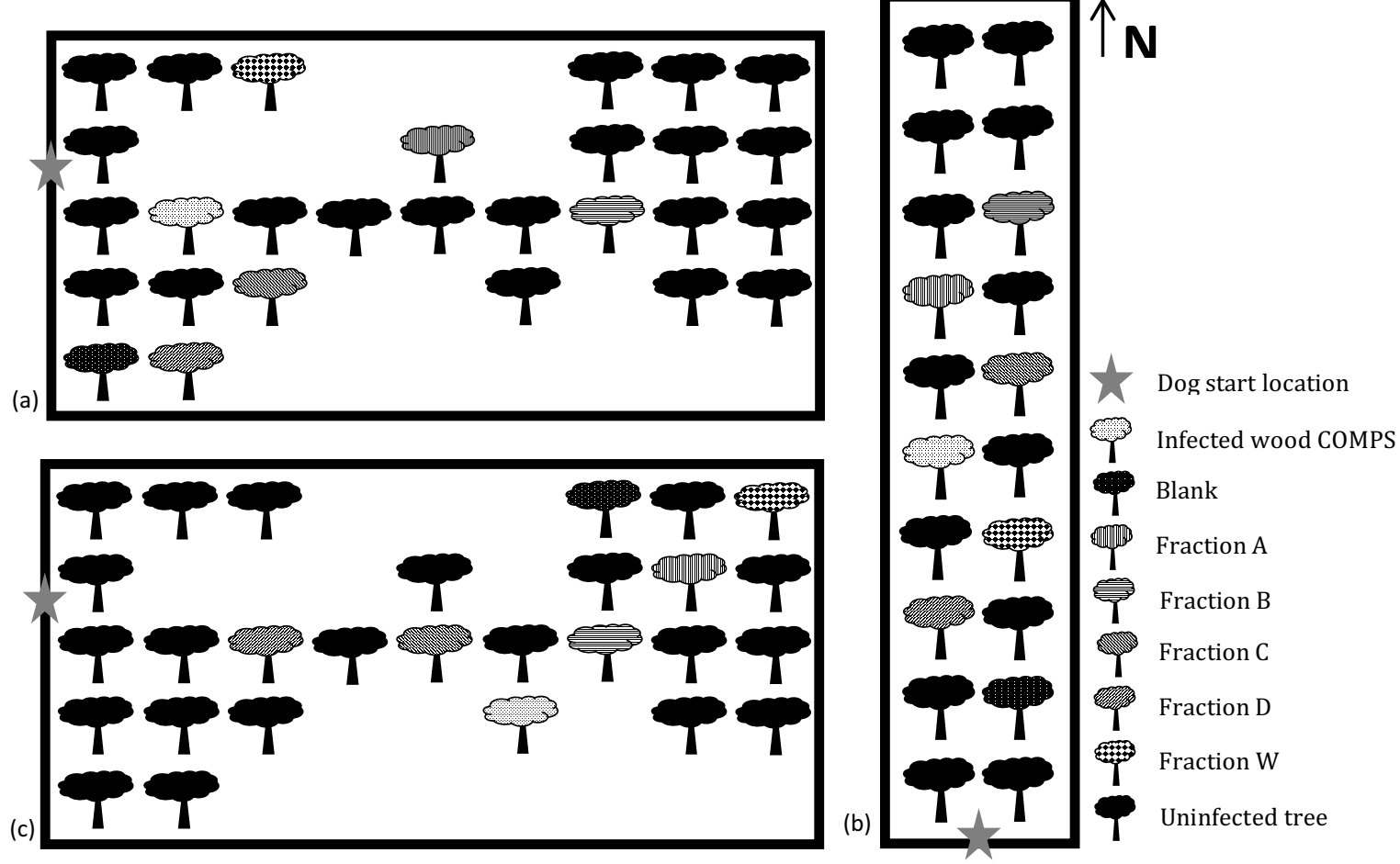

Figure 15: Design of canine experiments over three days. (a) Took place in a mango grove with 6mph (10kph) $\mathrm{SE}$ winds. (b) Took place in a longan grove with $10 \mathrm{mph}(16 \mathrm{kph}) \mathrm{NE}$ winds. (c) Took place in a mango grove with $15 \mathrm{mph}(24 \mathrm{kph})$ wind gusts in the $\mathrm{SW}$ direction.

The optimization of the experiment was performed with a $100 \mathrm{ppm}$ standardized solution of eight compounds that elute with retention times across all four fractions. The purpose of using standards for optimization was to ensure the collected fractions were separated properly. The percent loss was calculated for each compound by first injecting $1 \mu \mathrm{L}$ of $100 \mathrm{ppm}$ liquid solution in the $0.25 \mathrm{~mm}$ ID solgel-wax column and then performing the column vent method using the $0.53 \mathrm{~mm}$ ID solgel-wax column. These percent losses are given in Table 9. Percent loss for the compound in Fraction A was 43.78\%. In Fraction $\mathrm{B}$, the percent loss was $61.71 \%$. For Fraction $\mathrm{C}$, the percent loss ranged from $73.43 \%$ 91.58\% and for Fraction D, the percent loss ranged from $94.53 \%$ to $96.31 \%$. 
Table 9: Percent loss between direct liquid injection on $0.25 \mathrm{~mm}$ ID solgel-wax column and SPME of headspace of cotton gauze after collection from vented $0.53 \mathrm{~mm}$ ID solgel-wax column for each compound in a $100 \mathrm{ppm}$ standard solution.

\begin{tabular}{|l|l|r|r|}
\hline \multicolumn{1}{|c|}{ CAS No } & Compound & RT (min) & Percent Loss (\%) \\
\hline $123-92-2$ & 1-Butanol, 3-methyl-, acetate & 6.452 & 43.78 \\
\hline $100-52-7$ & Benzaldehyde & 18.767 & 61.71 \\
\hline $87-44-5$ & Caryophyllene & 20.529 & 73.43 \\
\hline $470-40-6$ & Thujopsene & 21.209 & 73.77 \\
\hline $25246-27-9$ & Alloaromadendrene & 21.964 & 79.76 \\
\hline $103-45-7$ & Acetic acid, 2-phenylethylester & 29.172 & 91.58 \\
\hline $60-12-8$ & Phenylethyl alcohol & 31.294 & 94.53 \\
\hline $1139-30-6$ & Caryophyllene oxide & 32.276 & 96.31 \\
\hline
\end{tabular}

\subsubsection{Characterization of the Canine Training Aid Used in the Field}

Canine training aids for the laurel wilt disease were in the form of COMPS used to contain the fungal spores while simultaneously releasing the active odorants. For these experiments, COMPS were created using the field method designed by the canine trainers since the purpose was to evaluate the field efficacy parameters: shelf-life and dissipation rate. The COMPS were created using 3"x3" 4MIL LDPE bags. Each bag was weighed to contain 10.0 grams of wood, then heat sealed across the top.

\subsubsection{Externally Sampled Internal Standard Optimization}

A method called externally sampled internal standard (ESIS) was implemented for SPME quantification ${ }^{125,126}$, and is described in Section 2.7.1. The DVB/CAR/PDMS SPME fibers were exposed to $5 \mu \mathrm{L}$ of a $10 \mathrm{ppm}$ mixture of naphthalene- $\mathrm{D}_{8}$ in methylene chloride (Sigma-Aldrich, St. Louis, MO) (made daily) in $10 \mathrm{~mL}$ clear glass vials with PTFE/silicone septa screw caps for 2.5 minutes, then immediately exposed to a COMPS enclosed in a sterilized 8oz. mason jar for one hour. Naphthalene- $\mathrm{D}_{8}$ was chosen because 
it was similar in molecular structure and vapor pressure, yet baseline separated on the GC to other target analytes (Table 10). The ESIS-SPME method was optimized by testing the following extraction times for the internal standard: $1,2.5,5,7.5$, and 10 minutes using standard solutions of $10 \mathrm{ppm}$ naphthalene- $\mathrm{D}_{8}$ and 10ppm 2-phenethyl acetate, 2phenylethanol, benzaldehyde, isoamyl acetate, thioacetic acid, $\beta$-caryophyllene, (-)caryophyllene oxide, (-)-thujopsene, (-)-alloaromadendrene, and $\alpha$-pinene in methylene chloride. 
Table 10: Relevant information for naphthalene- $D_{8}$ and examples of target compounds, including vapor pressure and molecular structure.

\begin{tabular}{|c|c|c|c|c|}
\hline CAS No. & Compound & Weight $(\mathrm{g} / \mathrm{mol})$ & Vapor Pressure $\left(\mathrm{mmHg}\right.$ at $\left.25^{\circ} \mathrm{C}\right)$ & Structure \\
\hline $1146-65-2$ & Naphthalene-D8 & 136.22 & 0.030 & \\
\hline $3856-25-5$ & Copaene & 204.35 & 0.038 & \\
\hline $483-76-1$ & $\delta$-Cadinene & 204.35 & 0.007 & \\
\hline $483-77-2$ & Calamenene & 202.34 & 0.005 & \\
\hline $87-44-5$ & $\beta$-Caryophyllene & 204.35 & 0.013 & \\
\hline $25246-27-9$ & (-)-Alloaromadendrene & 204.35 & 0.923 & \\
\hline
\end{tabular}

\subsubsection{Static Testing}

Seven COMPS were created using the method described in Section 4.3.2: three containing infected wood (Simmonds variety), three containing healthy wood (Simmonds variety), and one blank (containing nothing). When not being tested, COMPS were suspended in a chemical hood with no air flow (Figure 16). Prior to fiber exposure, COMPS 
were allowed to equilibrate in the closed mason jars for one hour (Figure 17). COMPS were tested every 24 hours for eight days following the optimized ESIS-SPME method and the GC-MS parameters shown in Table 11 and Table 12 using an Agilent Technologies HP 6890 Series Gas Chromatography System/HP 5973 Mass Selective Detector with a 30m 0.25mm ID solgel-wax column and solvent delay from 0-2.5 minutes. The following were then monitored: identification of compounds released from the COMPS, the amount of each compound released from the COMPS, and the dissipation rate of each compound identified.

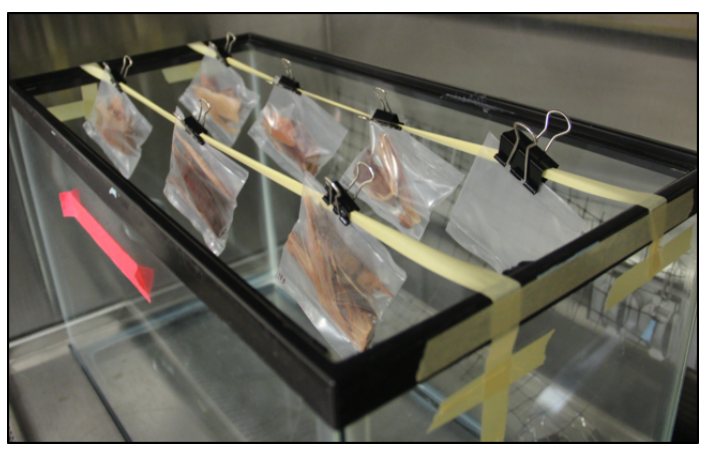

Figure 16: Image of COMPS suspended for static testing. 


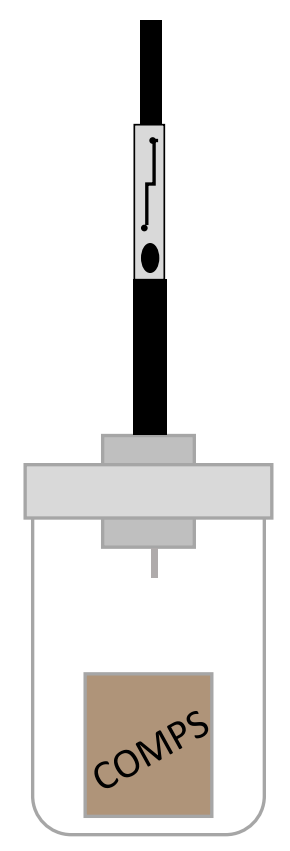

Figure 17: SPME sampling method for static testing (COMPS enclosed in a glass vial with a SPME fiber exposed above).

Table 11: The parameters for the Agilent Technologies HP 6890 Series Gas Chromatography System/HP 5973 Mass Selective Detector containing a $0.25 \mathrm{~mm}$ ID solgel-wax column.

\begin{tabular}{|l|r|}
\hline \multicolumn{2}{|c|}{ Parameters: $0.25 \mathrm{~mm} \mathrm{ID}$} \\
\hline Split Ratio: & $1: 1$ \\
\hline Desorption: & $15 \mathrm{~min}$ \\
\hline Low Mass: & $45.00 \mathrm{amu}$ \\
\hline High Mass: & $410.00 \mathrm{amu}$ \\
\hline Injection Temp: & $250^{\circ} \mathrm{C}$ \\
\hline Flow rate: & $1.0 \mathrm{~mL} / \mathrm{min}$ \\
\hline
\end{tabular}

Table 12: The temperature ramp used for the GC-MS analyses using an Agilent Technologies HP 6890 Series Gas Chromatography System/HP 5973 Mass Selective Detector (Santa Clara, CA).

\begin{tabular}{|r|r|r|r|}
\hline Temperature $\left({ }^{\circ} \mathrm{C}\right)$ & Rate $\left({ }^{\circ} \mathrm{C} / \mathrm{min}\right)$ & Hold $(\mathrm{min})$ & Total $(\mathrm{min})$ \\
\hline 50 & 0.0 & 5.00 & 5.00 \\
\hline 80 & 15.0 & 5.00 & 12.00 \\
\hline 150 & 10.0 & 5.00 & 14.00 \\
\hline 260 & 20.0 & 0.00 & 29.50 \\
\hline
\end{tabular}




\subsubsection{Dynamic Testing}

In the dynamic testing experiment, the storage was designed to mimic field use and the testing was designed to mimic canine exposure. Six COMPS were created using the method described in Section 4.3.2: five containing infected wood (Simmonds variety) and one blank (containing nothing). When not being tested, COMPS were stored in a sealed aluminum bag, which allows equilibration between bags and minimizes odor permeation. The bag was then kept in a chemical hood with no air flow. The COMPS were tested every 24 hours for five days following the optimized ESIS-SPME method and the GC-MS parameters shown in Table 11 and Table 12 for an Agilent Technologies HP 6890 Series Gas Chromatography System/HP 5973 Mass Selective Detector with a 0.25mm ID solgelwax column and solvent delay from 0-2.5 minutes. The only difference in the ESIS-SPME method was that the COMPS were placed in unsealed sterilized 8oz. mason jars for sampling (Figure 18). Prior to fiber exposure, COMPS were allowed to equilibrate in the open mason jars for one hour. The following were then monitored: identification of

compounds released from the COMPS, the amount of each compound released from the COMPS, and the dissipation rate of each compound identified. 


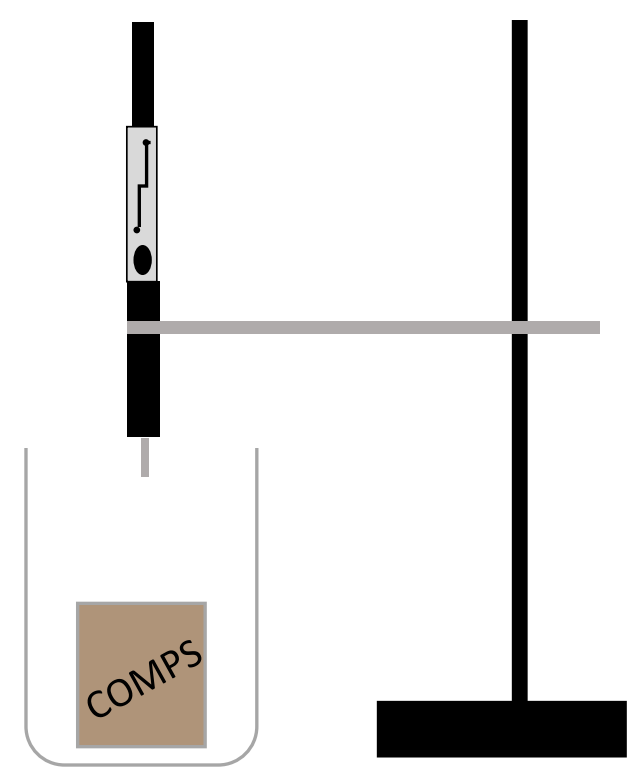

Figure 18: SPME sampling method for dynamic testing (COMPS in an open glass container with SPME fiber exposed above).

4.4. Task 4: Evaluation of the Volatile Organic Compounds of the Laurel Wilt Disease in Avocados Using Greenhouse Experiments

The purpose of Task 4 was to create a controlled environment in which the changes in VOCs produced by young avocado trees could be followed from before the time of inoculation through the initial disease cycle. The task was divided into two experiments. The first examined VOCs of trees inoculated with $R$. lauricola. The second examined VOCs of trees inoculated with $R$. lauricola and then treated with the fungicide, propiconazole. Both experiments have slightly different greenhouse designs, which are described in Sections 4.4.1 and 4.4.2. However, they follow the same laboratory protocols, explained here.

A sterilized blade was used to create a slight cut in each tree near the injection site to aid the release of volatiles. Then, 1-liter Tedlar whole-air sampling bags (SKC, Inc., 
Eighty Four, PA) were filled in the greenhouse using a vacuum pump (SKC, Inc., Eighty Four, PA) and taken to the lab. Bags were flushed with UPH nitrogen followed by ambient air prior to collection. All trees were sampled in triplicate. Sample/extraction blanks were also collected. Infected trees, healthy trees, and treated trees utilized separate equipment. Each tree had assigned collection bags, and equipment was flushed or sterilized between each triplicate sample.

Samples were then analyzed using HS-SPME-GC-MS. The DVB/CAR/PDMS SPME fibers were exposed within each bag for two hours at room temperature and humidity (recorded at the beginning of each fiber exposure; Appendix Table 18 and Table 19). The fibers were then desorbed for 15 minutes following the parameters in Table 11 and Table 12 for an Agilent Technologies HP 6890 Series Gas Chromatography System/HP 5973 Mass Selective Detector with a $0.25 \mathrm{~mm}$ ID solgel-wax column and solvent delay from 0-2.5 minutes.

At the conclusion of each study, about $20 \mathrm{~cm}$ of each tree from around the injection site was collected and prepared for SEM. The remainder of the trees was burned to prevent the spread of spores. To preserve the samples, $1 \mathrm{~cm}$ transverse sections of each tree were placed in $2 \%(v / v)$ glutaraldehyde in $0.1 \mathrm{~mol} / \mathrm{L}$ phosphate buffer $(\mathrm{pH} 7.2)$. After being frozen overnight at $1{ }^{\circ} \mathrm{C}$, the samples were placed in $25 \%$ tissue freezing medium. To prepare for SEM, the $1 \mathrm{~cm}$ sections were dried overnight in a vacuum dryer and then sputtercoated (Hummer ${ }^{\circledR} 10.2$ Sputtering System, Anatech Ltd., Battle Creek, MI) for 4 minutes with gold at $367 \AA \mathrm{Au} / 2 \mathrm{~min}$. Glutaraldehyde and phosphate buffer saline were purchased from Sigma-Aldrich (St. Louis, MO), and the tissue freezing medium was purchased from PSL Equipment, Inc. (Vista, CA). 
4.4.1. Examining the Volatile Organic Compounds of Avocado Trees Inoculated with Raffaelea lauricola

Ten Lula variety avocado trees (Pine Island Nursery, Inc., Miami, FL) averaging $0.9 \mathrm{~m}$ tall and $1.5 \mathrm{~cm}$ in diameter at injection site were kept either inside a greenhouse or outside along the greenhouse wall facing west. Five trees were inoculated with fungal spores (stored inside along with two control trees). The remaining three control trees were stored outside. Temperature and humidity were collected every 30 minutes in both locations for each day of the study (Appendix Figure 62 and Figure 63, respectively).

For inoculation, a $2 \mathrm{~mm}$ hole was drilled into each tree $15 \mathrm{~cm}$ above the graft site, respective solutions were introduced, and the site was wrapped in Parafilm. For the five inoculated trees, a prepared suspension of $50 \mu \mathrm{L}$ of $2 \times 10^{6}$ spores $/ \mathrm{mL}$ was injected. Spores were received from the University of Florida's Tropical Research and Education Center (Homestead, FL). For the control trees, $50 \mu \mathrm{L}$ of DI water was injected. Samples were collected days $0,1,3$, and every 3 days thereafter for a total of 20 sample days. Two weeks prior to the initiation of the study, the greenhouse was treated with RoundUp (herbicide) and malathion (aphid killer).

\subsubsection{Examining the Volatile Organic Compounds of Treated Infected Avocado Trees}

Eleven Lula variety avocado trees (Pine Island Nursery, Inc., Miami, FL) averaging $0.5 \mathrm{~m}$ tall and $1.3 \mathrm{~cm}$ in diameter at injection site were kept either inside a greenhouse or outside along the greenhouse wall facing west. Four trees were inoculated and left untreated (stored inside). Four trees were inoculated and later treated with propiconazole 
fungicide (Dorado ${ }^{\circledR}$, Greensboro, NC) (stored inside). The remaining three trees served as control trees and were stored outside until day 17 , at which point they were moved inside to prevent theft (only two control trees remained for day 17 through the end of the study). Temperature and humidity were collected every 30 minutes in both locations for each day of the study (Appendix Figure 64 and Figure 65, respectively).

For inoculation, a $2 \mathrm{~mm}$ hole was drilled into the trees $15 \mathrm{~cm}$ above the graft site, respective solutions were introduced, and the site was wrapped in Parafilm. For the eight inoculated trees, a prepared suspension of $50 \mu \mathrm{L}$ of $2 \times 10^{6}$ spores $/ \mathrm{mL}$ was injected. Spores were received from the University of Florida's Tropical Research and Education Center (Homestead, FL). For the control trees, $50 \mu \mathrm{L}$ of DI water was injected. On day 7, five injections of $200 \mu \mathrm{L}$ of propiconazole were infused around the flare roots of each of four inoculated trees as well as the three control trees. The $1000 \mu \mathrm{L}$ infusion amount was chosen using the method applied in the field for macroinfusion of the fungicide (Figure 19). Samples were collected days $0,1,3,5,7,8,10$, and every 3 days thereafter for a total of 13 sample days. 


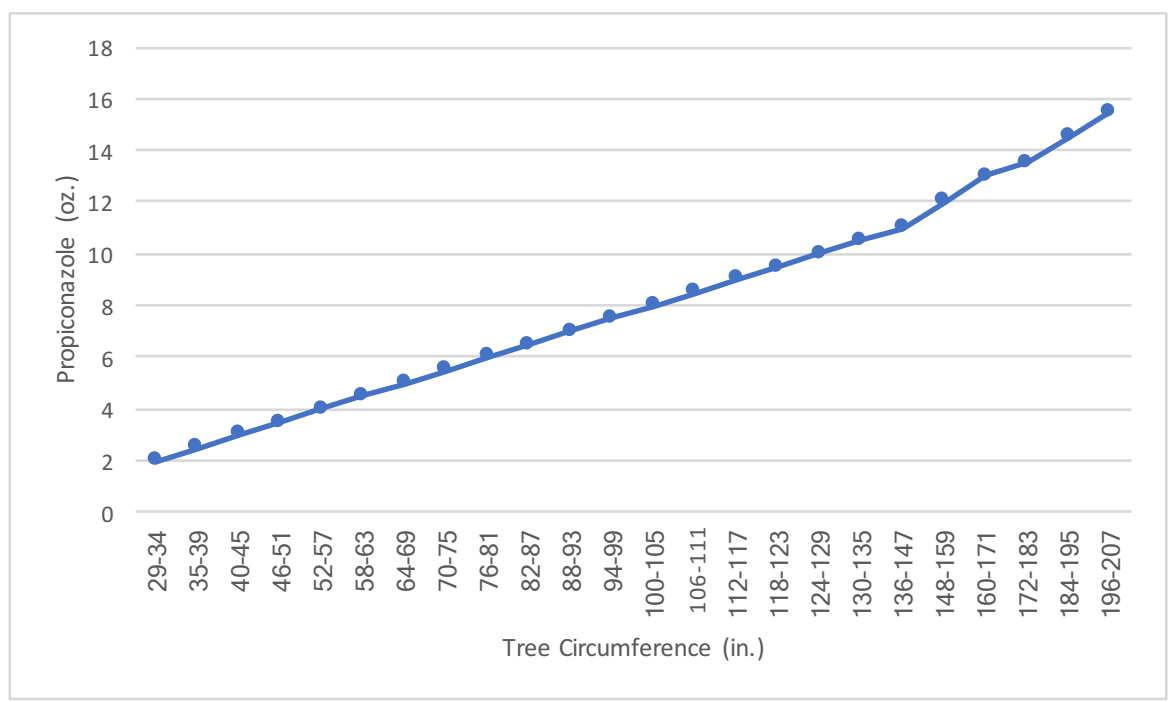

Figure 19: Field guide for avocado tree macroinfusion with propiconazole.

\section{RESULTS AND DISCUSSION}

The section presents the results and discussions for each task and research objective of the dissertation listed in Section 3.

5.1. Task 1: Optimization of a Method for the Detection of the Volatile Organic Compounds Released from the Laurel Wilt Disease

The purpose of Task 1 was to optimize a method which can be applied to experiments throughout the research. It took place in three portions: SPME fiber selection (Section 5.1.1), SPME fiber exposure time selection (Section 5.1.2), and creation of appropriate GC-MS methods (Section 5.1.3). 


\subsubsection{Solid Phase Microextraction Fiber Selection}

Each of four SPME fiber coatings were tested using seven analytical standards as described in Section 4.1.1. While the differences were not considered statistically significant, the results (Figure 20) showed that DVB/CAR/PDMS resulted in both the highest amount recovered and the lowest standard deviation for each compound evaluated.

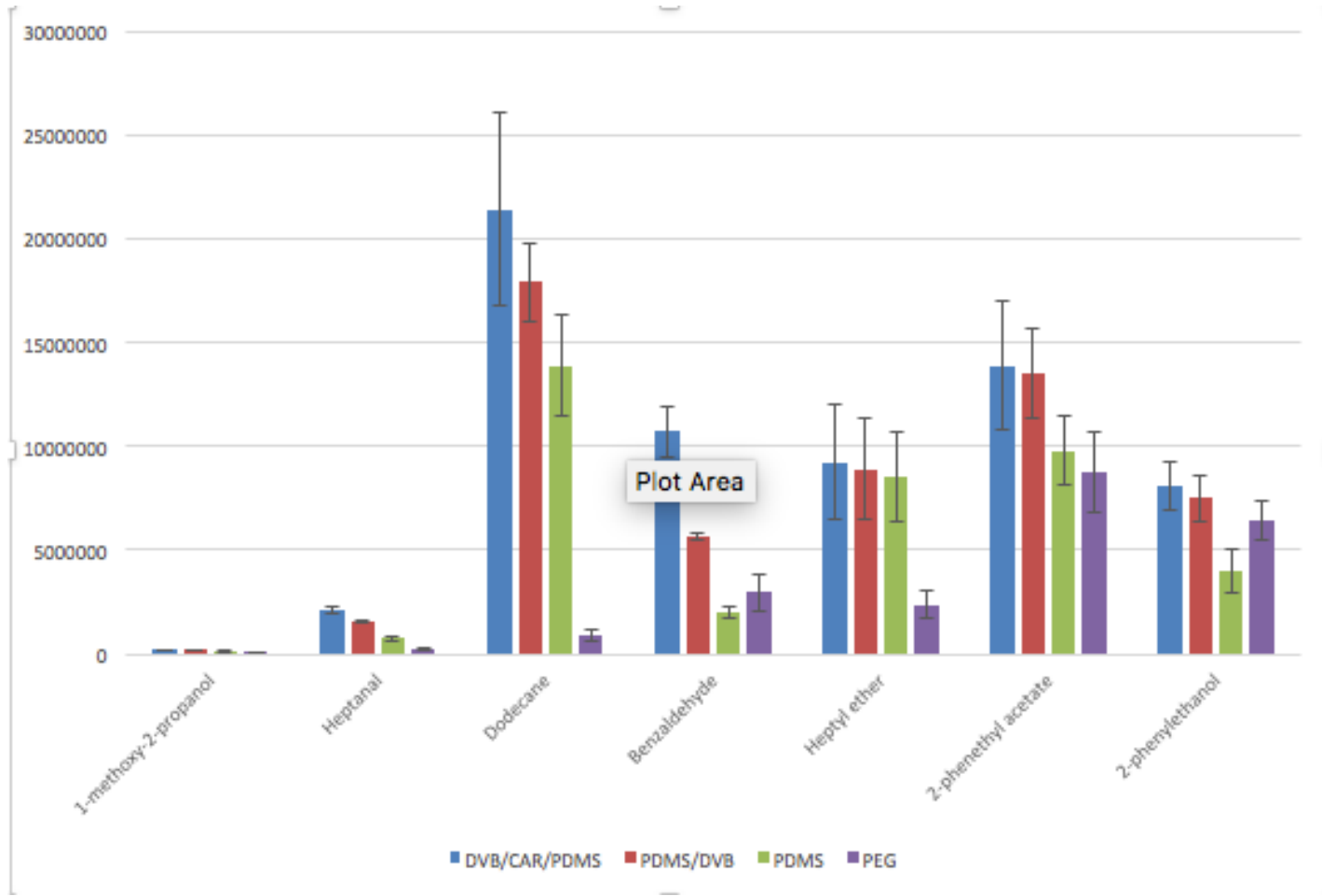

Figure 20: Peak areas of compounds recovered from SPME extraction using various fiber chemistries.

\subsubsection{Extraction Time Study}

Each of five extraction times were tested using the selected DVB/CAR/PDMS fiber coating using seven analytical standards as described in Section 4.1.2. The differences were not considered statistically significant, but since the results (Figure 21) showed slightly 
lower standard deviations for the one hour extraction time, this was chosen as the fiber exposure time for future studies.

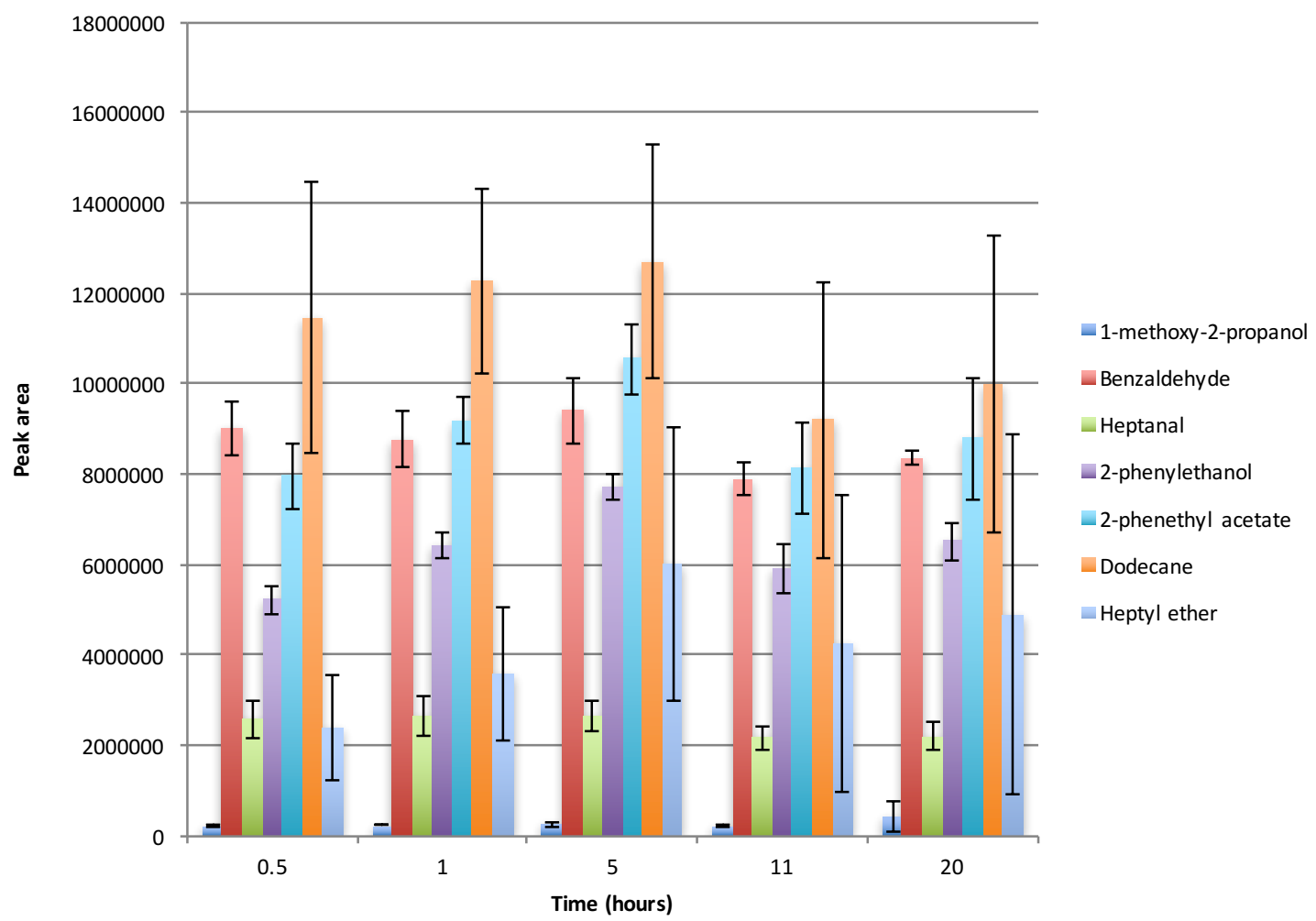

Figure 21: Peak areas of compounds recovered from SPME extraction using various extraction times.

For the externally sampled internal standard-solid phase microextraction (ESISSPME) method utilized in Section 4.3.2, extraction time was optimized for fiber exposure to naphthalene- $\mathrm{D}_{8}$. Again, five extraction times were tested using nine analytical standards. The results are shown in Figure 22. Exposure for 2.5 minutes was chosen because it displayed the lowest standard deviation with the best extraction for all tested compounds. 


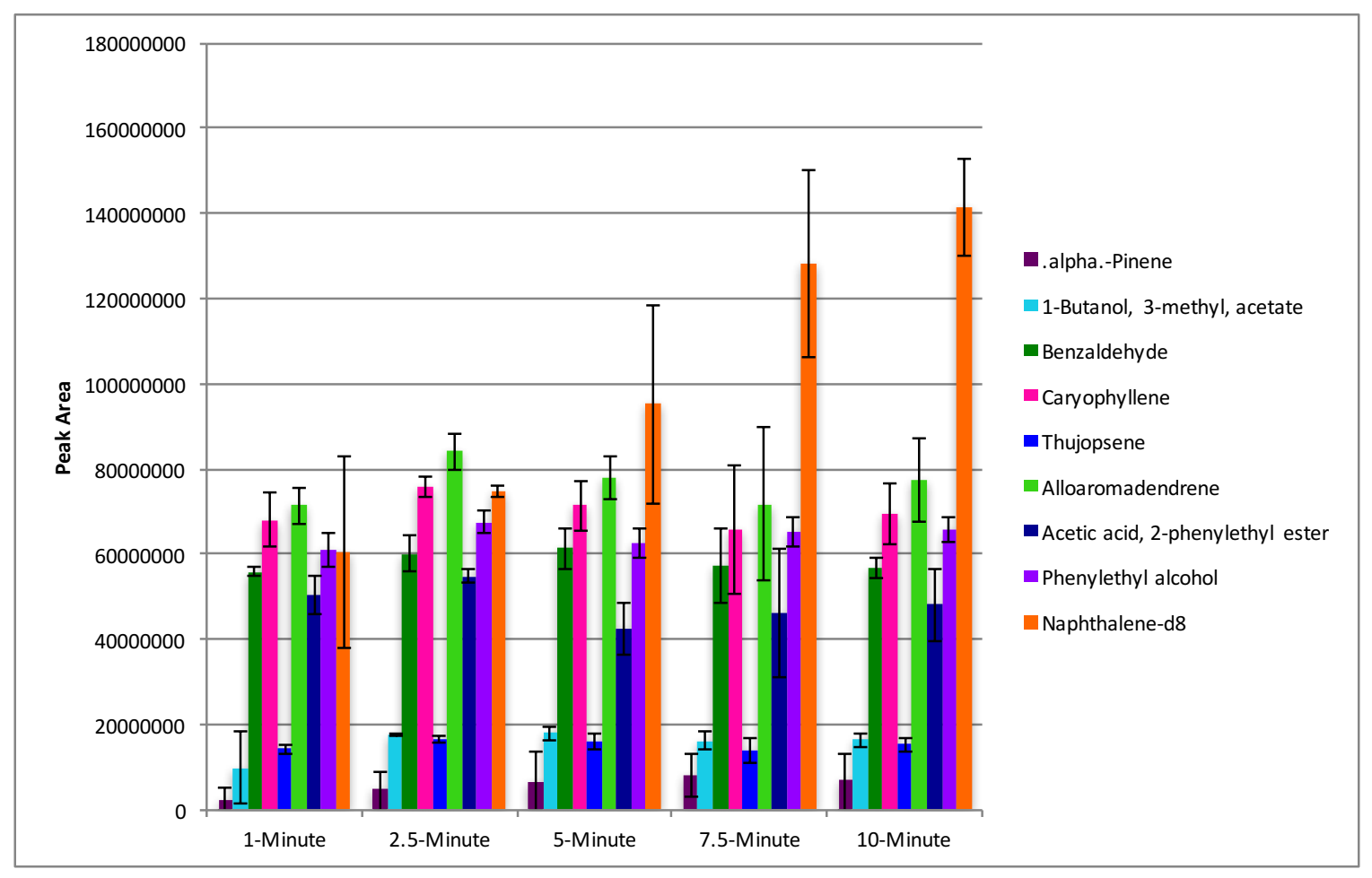

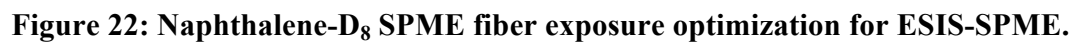

\subsubsection{Gas Chromatography-Mass Spectrometry Optimization}

The GC-MS methods for both the Varian 3800 Gas Chromatograph/Saturn 2000 Ion Trap Mass Spectrometer and the Agilent Technologies HP 6890 Series Gas Chromatography System/HP 5973 Mass Selective Detector was done using analytical standards as described in Section 4.1.3. The temperature ramp, solvent delay, desorption temperature, and desorption time were tested, and the results are given in Table 5, Table 6, Table 7, Table 8, Table 11, and Table 12 for each respective instrument and evaluated parameter. 


\subsection{Task 2: Analytical Studies of the Laurel Wilt Disease}

The purpose of Task 2 was to identify the VOCs of several systems. The first to be examined was the fungus $R$. lauricola. Fungal VOCs are biologically dynamic and are therefore affected by various factors, such as the media on which it is grown, the duration of incubation, the type of nutrients it can access ${ }^{12}$, the temperature of the environment it is grown in ${ }^{12,23}$, and various other environmental conditions. These factors must be considered when identifying the VOCs of the laurel wilt pathogen that are present as it grows in avocado trees. Because detection canines are targeting early infection, prior to the occurrence of visual symptoms, it is important to understand the fluctuation of VOCs during the initial period of incubation. Avocado trees begin displaying physical symptoms of infection around 2-4 weeks after inoculation with $R$. lauricola. The target detection frame is therefore approximately $0-4$ weeks. The results presented in Section 5.2.1 analyzed VOCs of the fungus grown on agar over a period of four weeks to assess any variation in the VOCs that are released in this time.

Secondly, the VOC production of adult infected avocado trees collected from groves in South Florida were examined. The odorants of these trees are the target for detection canines, so it is important to identify common VOCs of infected avocado trees, independent of location, time of day, weather factors, and variety. Section 5.2.2 addresses results related to these studies.

Finally, the VOC production of adult healthy, or uninfected, avocado trees collected from groves in South Florida was examined. The identification of volatiles was important information to utilize as controls in order to differentiate them from infected trees. Section 
5.2.3 assesses the similarities and differences between infected and healthy avocado trees that canines encounter in field work.

\subsubsection{Examining the Volatile Organic Compounds of Raffaelea lauricola Over Time}

Volatile organic compounds (VOCs) above the fungus $R$. lauricola were monitored over a 28 day period and analyzed using HS-SPME-GC-MS ${ }^{25}$. The VOC profiles for the observation period are displayed in Figure 23 showing the increases and decreases of each compound throughout the study. The compounds in Figure 23 were identified on the basis of fragmentation patterns and confirmed using a mass spectral library (NIST 2000 MS Search 2.0 and AMDIS, Gaithersburg, MD). The odor profiles showed that over the 28 day observation period, a total of seventeen individual compounds were detected using the current method.

The results demonstrated changes in fungal growth by the VOCs produced overtime (Figure 23). Days 1-14 present a similar odor profile. Over the period, the $\mathrm{C}_{8}$-compound 4,5-dimethyl-1-hexene was found to be the largest contributor to the odor profiles. Other contributors in this period include 4-ethenylidene-6,6-dimethylbicyclo[3.1.1]heptane; phenylethyl alcohol; isoamyl acetate; and methyl 4,6-dimethyloctanoate. Days 15-28 present a period of significant change in the odor profile of $R$. lauricola. During this time, 4,5-dimethyl-1-hexene ceased to appear in the odor profile, and was replaced by several different terpenes. Phenylethyl alcohol also disappears from the odor profile. Alcohols, acetates, and esters do not appear in the profile after Day 14, having been replaced by various terpenes. 


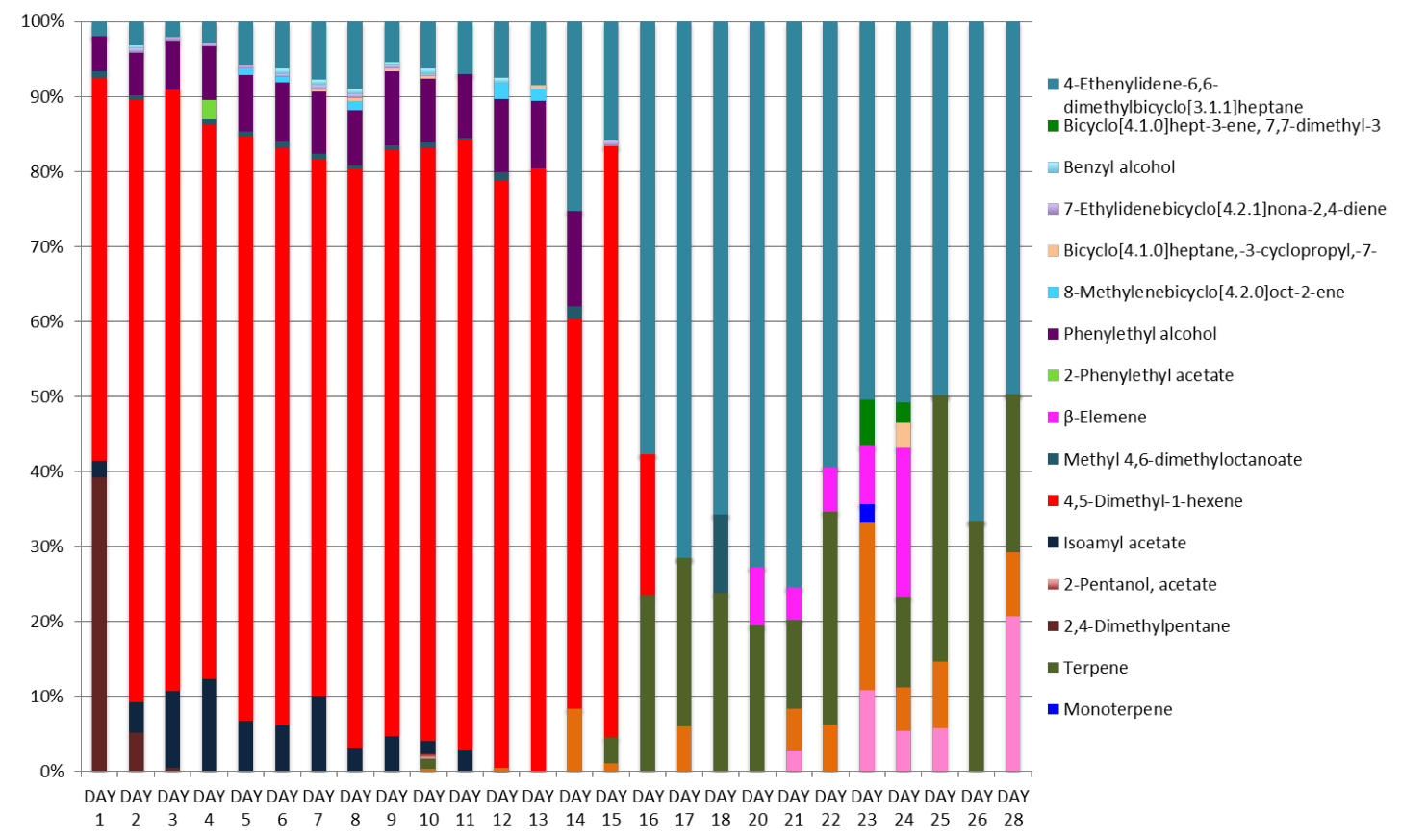

Figure 23: VOC profiles of $R$. lauricola throughout observation period. No exact specification was possible for terpenes, and their identities as terpenes were based on mass spectral peaks.

Figure 23 showed the marked difference in the patterns of the odor profiles between Days 1-14 and Days 15-28. To further characterize these differences, XCMS Online was used to produce a PCA and cloud plots ${ }^{137}$. In Figure 24, the PCA shows clustering of days with similar compounds. Separation was achieved through multi-group comparison analysis, where the data were grouped into three sets: Days 1-14, Days 15-20, and Days 21-28 (Figure 24). The first principle component (PC1) explained 30\% of variance (cumulative variance of 0.5997 ) and PC2 is based on a variance of $12 \%$ (cumulative variance of 0.7620). The group Days 1-14 was separated from Days 21-28 using PC2, but it is separated from Days 15-20 based on PC1. Additionally, Days 15-20 was separated from Days 21-28 based on PC1 and PC2. 


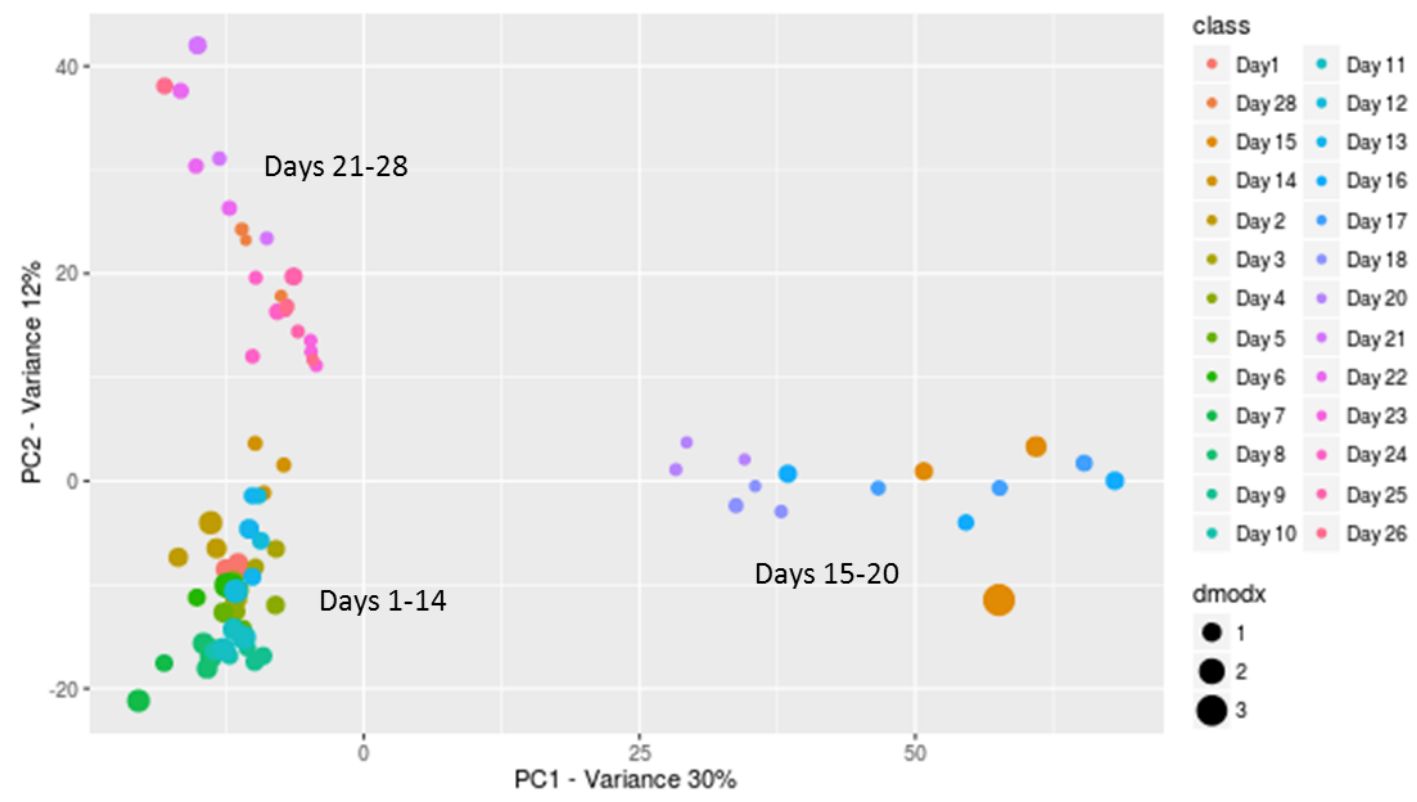

Figure 24: PCA produced by XCMS Online showing separation in datasets by day.

Fungal VOCs like those detected in Days 1-28 are produced by cell metabolism and fungal growth (Figure 1). Primary metabolism describes the chemical processes in organisms that are necessary for life, while secondary metabolism describes specialized pathways that aid in survival but are not required to sustain organismal growth. For example, terpene biosynthesis occurs in the mevalonate acid (MVA) pathway, ${ }^{5,6}$ a secondary metabolic pathway stemming from acetate coenzyme A (acetyl-CoA). Isopentyl diphosphate and its isomer dimethylallyl diphosphate (DMAPP) form isoprene units that serve as the building blocks for terpenes. The formation of secondary metabolites, such as terpenes, is associated with morphological developments after active growth has ended ${ }^{6,8,9}$.

The separation of the three groups seen in Figure 24 is likely as a result of shifts in the VOCs of the fungus in different stages of growth. In the initial stages of growth, the tricarboxylic acid (TCA) cycle is more productive than other metabolic cycles and 
produces primary metabolic compounds. Furthermore, secondary metabolic compounds are produced by the MVA pathway. It was shown in controlled greenhouse experiments that an avocado tree's response to infection with $R$. lauricola begins during the first 14 days after inoculation ${ }^{34,36}$. While secondary metabolites were detected in Days 15-28, it should be noted that the separation between Days 15-20 and Days 21-28 seen in Figure 24 is a result of more diverse terpene production during Days 21-28 than in the middle phase (Days 15-20). The differences in VOC production over the 28 day period indicates that fungal growth is not static, as the number of VOCs detected increased and varied over time. A cloud plot showed both fold change in ion intensity and level of significance for these changes ${ }^{137}$. Significant ion intensities in detected metabolite features above aligned chromatograms were plotted based on retention time (minutes) on the X-axis and $\mathrm{m} / \mathrm{z}$ on the y-axis. The cloud plot in Figure 25 showed 236 detected metabolite features with pvalue $\leq 0.01$ and fold change $\geq 1.5$. Toggle upregulated and downregulated features are differentiated based on color, where green demonstrates toggle upregulation, or fold increases, and red demonstrates toggle downregulation, or fold decreases. The intensity of the color of each circle indicates the statistical significance of each feature difference, so that low p-values are represented by darker colors. The radii of the circles indicate the logfold increase or decrease in abundance, where larger circles indicate larger fold changes. Fold change ranged from 1.5 to 3563.968 and $\mathrm{m} / \mathrm{z}$ ranged from 0 to 311 . 


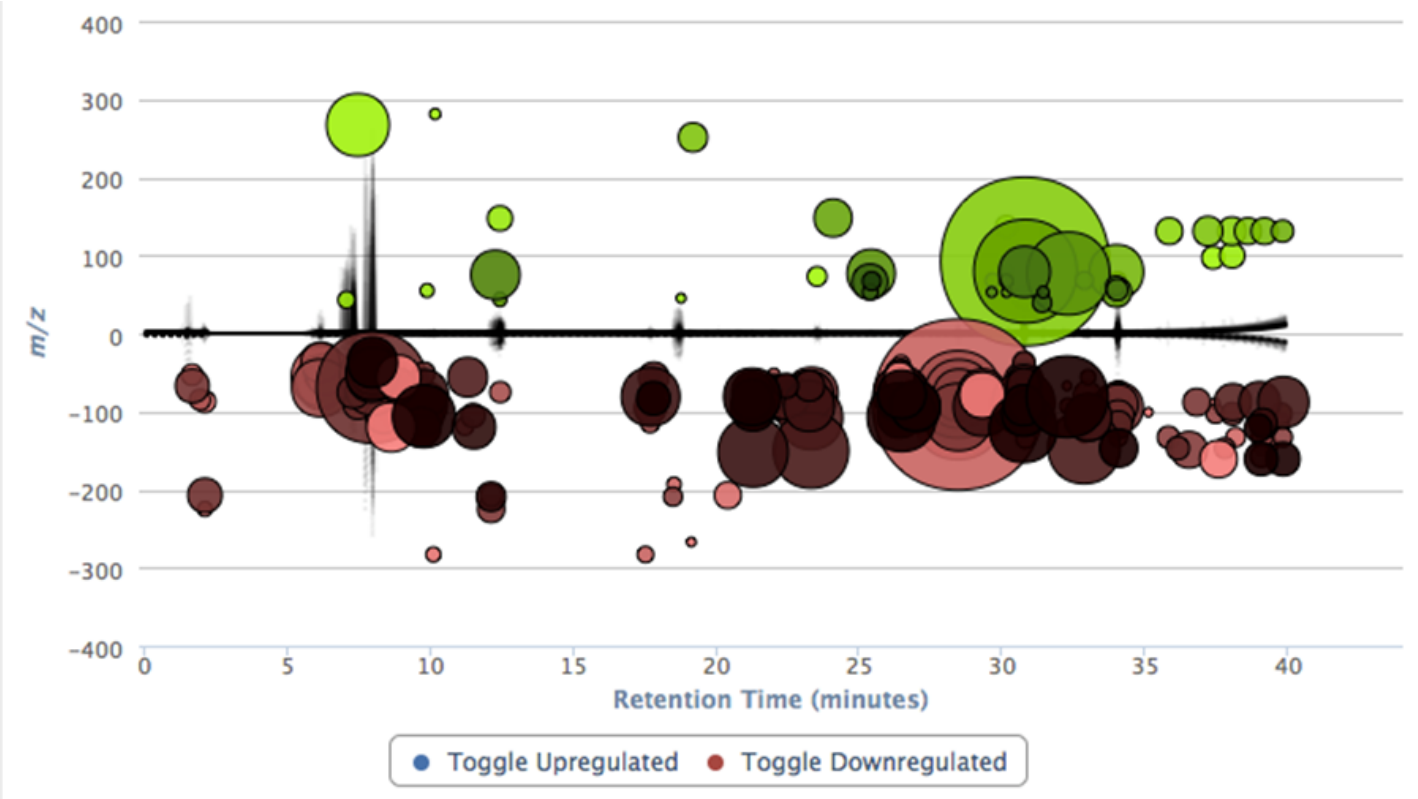

Figure 25: Cloud plot produced by XCMS Online showing separation in Days 1-14 (upper TIC) and Days 15-28 (lower TIC) by presenting aligned chromatograms (TIC) plotted based on retention time (minutes) on the $x$-axis and $\mathrm{m} / \mathrm{z}$ on the $\mathrm{y}$-axis. The circles represent detected metabolite features with $\mathrm{p}$-value $\leq \mathbf{0 . 0 1}$ and fold change $\geq$ 1.5. Green circles indicated toggle upregulated features, while red circles indicate toggle downregulated features. Low p-values are represented by darker colors and larger circle radii indicate larger fold changes.

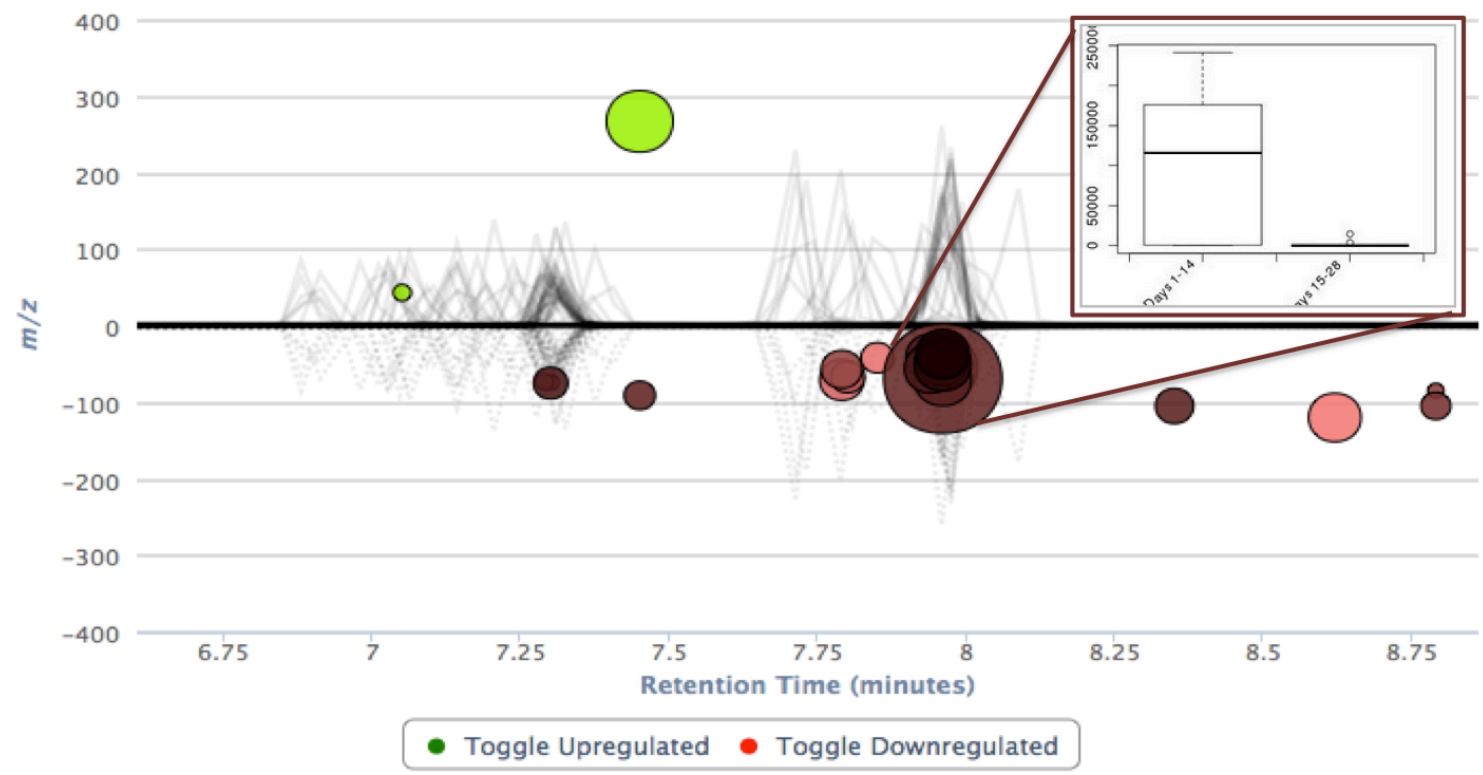

Figure 26: Retention time (minutes) 6.25 through 9 (zoom in from Figure 25) from cloud plot produced by XCMS Online with a box-and-whisker plot showing the toggle downregulation of the feature identified using a mass spectral library as 4,5-dimethyl-1-hexene. 


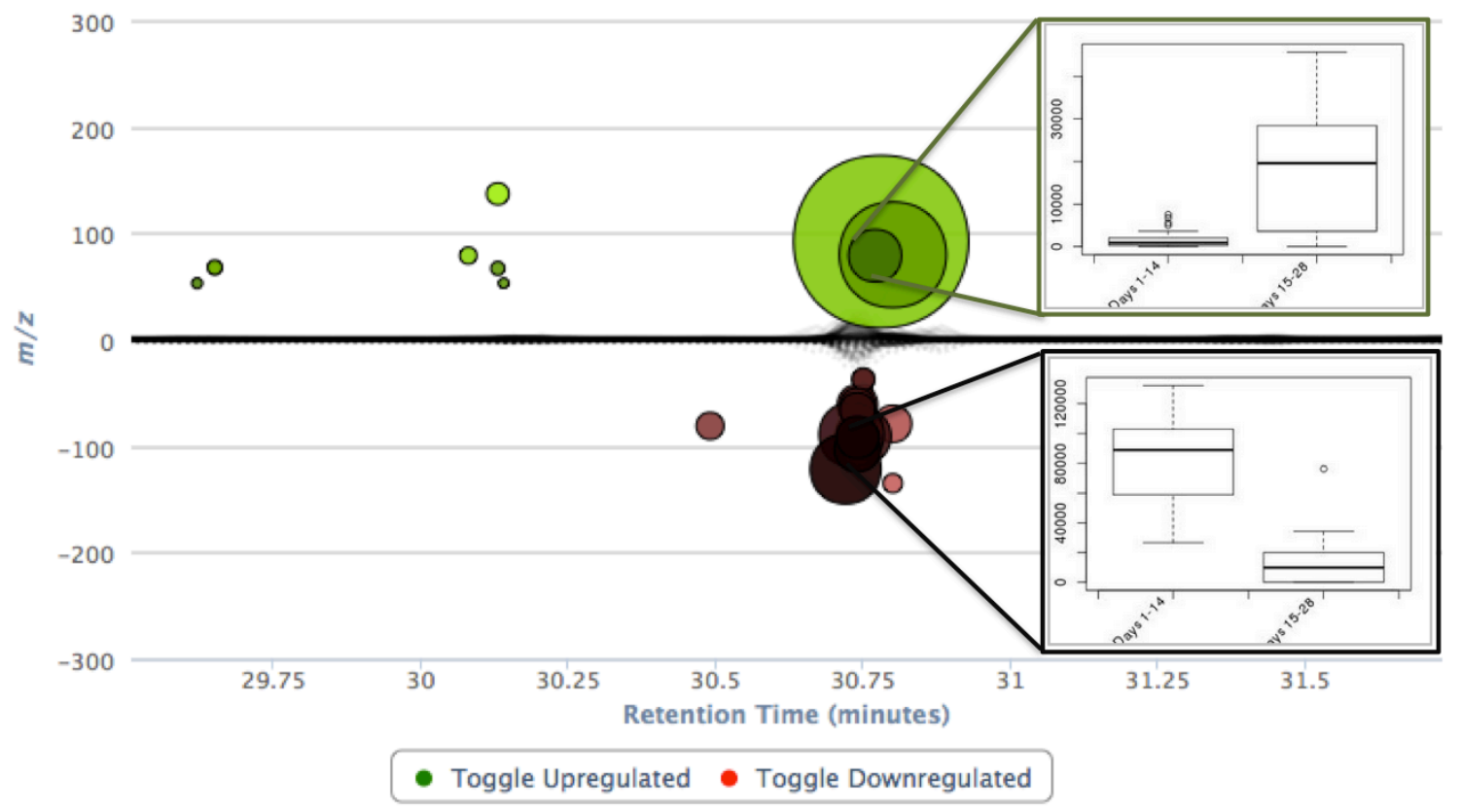

Figure 27: Retention time (minutes) 29.5 through 31.75 (zoom in from Figure 25) from cloud plot produced by XCMS Online with a box-and-whisker plot showing the toggle downregulation of the feature identified using a mass spectral library as phenylethyl alcohol and the toggle upregulation of the feature identified using a mass spectral library as a terpene.

The cloud plot in Figure 25 supports the original findings from Figure 23 regarding the increases (toggle upregulated) and decreases (toggle downregulated) of the seventeen identified compounds. For example, Figure 26 shows a box-and-whisker plot for one feature over a portion of Figure 25 (approximately minutes 6.5 through 9), revealing the toggle downregulation of 4,5-dimethyl-1-hexene (fold decrease=196.720). Toggle downregulation indicates that 4,5-dimethyl-1-hexene is not present in chromatograms after Day 14. Further, Figure 27 (approximately minutes 29.5 through 31.75 ) shows the box-andwhisker plots for phenylethyl alcohol and an unspecified terpene, which are toggle downregulated and upregulated, respectively. These two compounds elute at the same retention time (30.78 min), but phenylethyl alcohol is produced in the TCA cycle (Days 114) and the terpene is produced in a later stage of growth (Days 15-28). Phenylethyl alcohol 
(fold decrease $=6.821$ ) is toggle downregulated because it is present in the chromatograms in Days 1-14, but absent thereafter. The terpene (fold increase=11.236) is toggle upregulated because it is not present in the chromatograms until Day 15 and remains throughout the remainder of the study.

The VOCs produced by fungal cultures were the focus of the experiment because plants, fungi, and insects communicate and interact through the production of $\mathrm{VOCs}^{2,3,11,12}$. Therefore, knowing the VOCs produced by R. lauricola at initial inoculation may aid in comprehension of the host avocado tree's reaction as additional studies are completed. The 28 day observation period of $R$. lauricola VOCs displays the biological dynamicity of the organism's odor profile. The odor profile changed as the fungus entered different stages of growth, which change rapidly at the beginning of development, or Days 1-14. Terpenes, which appeared after the initial growth period, are produced from the secondary metabolism MVA pathway. The shift in VOC profiles after Day 14 (Figure 24) may have represented that active growth had declined and the fungus was undergoing sporulation. While previous literature ${ }^{6,8,21}$ and the data resulting from the study both support that these patterns occur in R. lauricola, additional studies should be done to confirm the findings.

The fungus $R$. lauricola grows rapidly, as seen by the secondary metabolic changes in VOC profiles observed in the study. Host trees both in the wild and in commercial groves in the United States have abrupt, exaggerated responses, possibly as a result of interactions between these changing $R$. lauricola VOCs and the host trees' VOCs. During the observation period, the primary metabolic compounds from Days 1-14 were toggle downregulated, while the MVA pathway developed into the dominant odor-producing pathway. Toggle upregulated terpenes and hydrocarbons became the dominating VOCs in 
the later stages of growth. These results show that the VOCs above R. lauricola are more complex than initially reported in the literature ${ }^{26}$.

Understanding the pattern of VOCs over the growth period for R. lauricola may enhance the ability to fight laurel wilt in the early growth phases by targeting the infection in the initial stage of development. The first six weeks after inoculation is also the time when a host tree's immune response recognizes the pathogen as an invader and begins to respond. It is possible that by delaying treatment until the fungus has reached sporulation, when the fungus' MVA pathway is dominant, laurel wilt disease has already begun and the tree is dying.

There is no current fungicide that can kill $R$. lauricola; however, Tilt巴 (propiconazole) is approved commercially for prevention ${ }^{106,107}$. It is possible that propiconazole is not fully effective as a treatment against the fungus because the inoculated tree has already recognized the pathogen as invasive through the fungal VOCs produced. As the xylem vessels are shut down, less fungicide can be transported to the site of the infection and thus the efficacy drops. It is also the only fungicide approved for treating the disease in avocado trees because it leaves no residual in the fruit. Therefore, the most effective treatment is likely a proactive approach that includes the use of plant activators to bolster the tree's system in addition to preventative fungicides. Future efforts should include ways to strengthen trees' defenses against the fungus prior to spore development. 


\subsubsection{Establishing Volatile Organic Compounds of Infected Avocado Trees}

Since media can affect VOC production, the study was conducted to explore the VOCs in the headspace of $R$. lauricola growing in avocado trees rather than PDA, as in the previous experiment. VOCs were detected in the headspace above Lula variety infected $(\mathrm{n}=15)$ avocado trees using HS-SPME-GC-MS. Compounds were considered to be present in a given variety of tree if it occurs in at least $80 \%$ of samples. Six compounds present in infected samples were positively identified and confirmed using either standard mixtures or a mass spectral library (NIST 2000 MS Search 2.0 and AMDIS, Gaithersburg, MD). While individual trees show greater variation in VOCs produced ${ }^{2,20,144-146}$, the compounds presented in Table 13 are the focus of the results presented here. These compounds included caryophyllene oxide, $\delta$-elemene, copaene, $\gamma$-muurolene, $\delta$-cadinene, and calamenene.

The six VOCs identified were all sesquiterpenes from the mevalonate acid (MVA) pathway, which produces secondary metabolites. They were categorized based on the terpene backbone stemming from different synthases leading to their formation. For example, copaene from the cadinenes backbone, the main X. glabratus attractant, is formed from $\delta$-cadinene catalyzed by $(+)-\delta$-cadinene synthase ${ }^{147}$. The categorization was done because the parent compounds provide more information about the trees' reactions to infection than the individual compounds. Additionally, only one of the compounds has a commercially available standard. Five different backbones were identified: eudesmenes; guaienes; germacrenes; cadinenes, muurolenes, \& calamenenes; and humulenes (Table 13). The various ring closures identified can be seen in Figure 28, and are detailed herein (Figure 29, Figure 30, Figure 31, Figure 32, Figure 33, Figure 35, Figure 36, and Figure 37). 
Table 13: Compounds present in at least $80 \%$ of Lula variety avocado tree samples. Presence in infected for uninfected trees is marked.

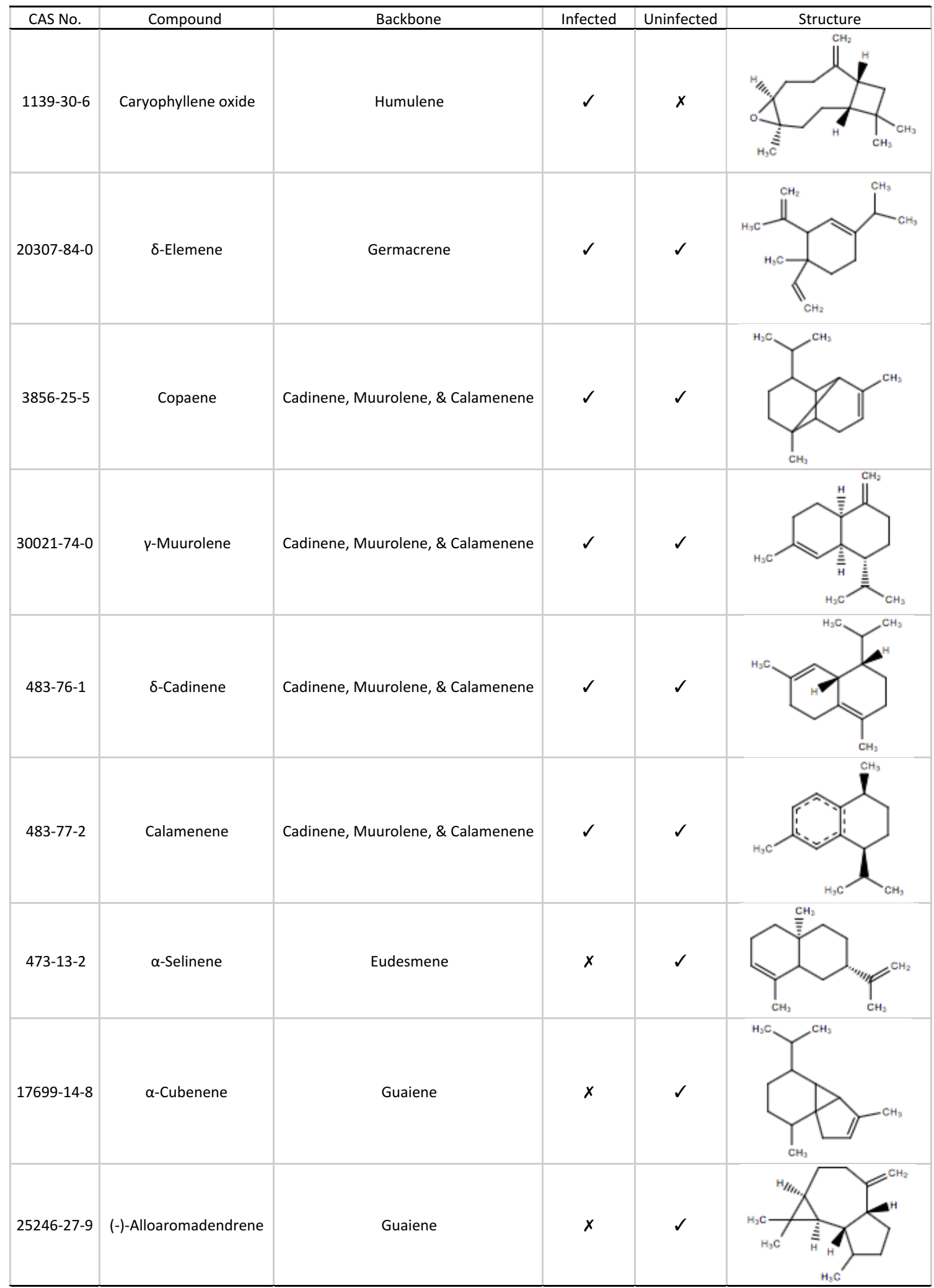




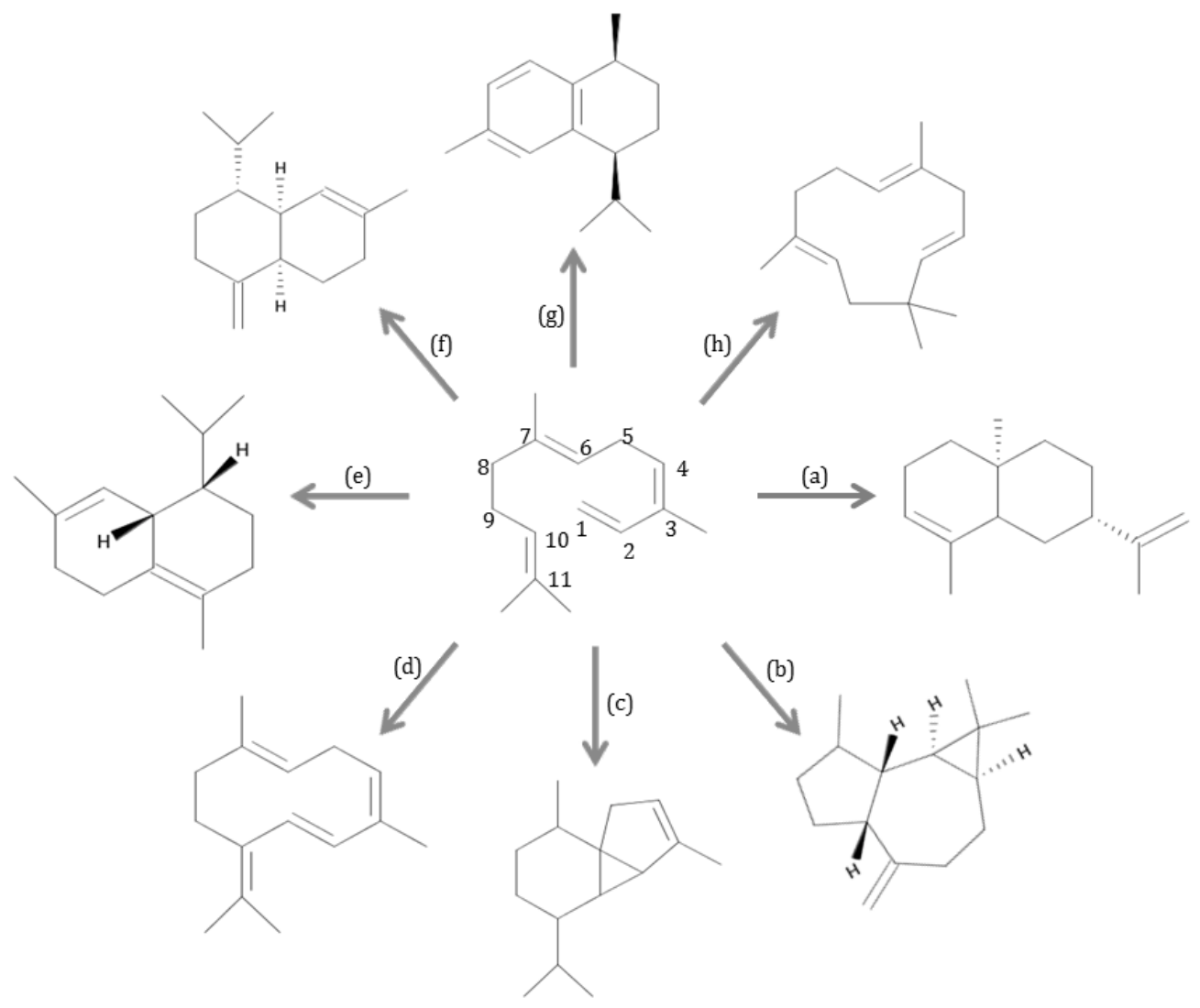

Figure 28: Various ring closures of farnesene (center molecule) leading to nine identified VOCs in avocado wood samples, where (a) is the eudesmene backbone; (b) is the guaiene backbone; (c) is the germacrene backbone; (d)(g) are is the cadinene, muurolene, $\&$ calamenene backbone; and (h) is the humulene backbone.

Ring closures of C-1 and C-6 plus C-5 and C-10 lead to copaene (Figure 29), $\gamma$ muurolene (Figure 30), $\delta$-cadinene (Figure 29), and calamenene (Figure 31). Bond formation between $\mathrm{C}-2$ and $\mathrm{C}-7$ on the $\delta$-cadinene molecule forms copaene, which is thought to be the primary attractant for the redbay ambrosia beetle ${ }^{18,20}$. The stereoisomer of copaene is known as ylangene. Copaene was present in $100 \%$ of infected samples and $89 \%$ of uninfected samples, while ylangene was only present in $73 \%$ of infected samples and $67 \%$ of uninfected samples. Though not always present, ylangene was never present without copaene. $\gamma$-Muurolene was present in $87 \%$ of infected samples and $100 \%$ of uninfected 
samples. However, muurolene was present in $53 \%$ of infected samples, but $0 \%$ of uninfected samples. It can thus be concluded that some of the $\gamma$-muurolene forms muurolene because of the infected trees' stress and resulting increase in energy expelled fighting the disease. The calamenene molecule contains one benzenoid ring, which was unique in this ring closure.

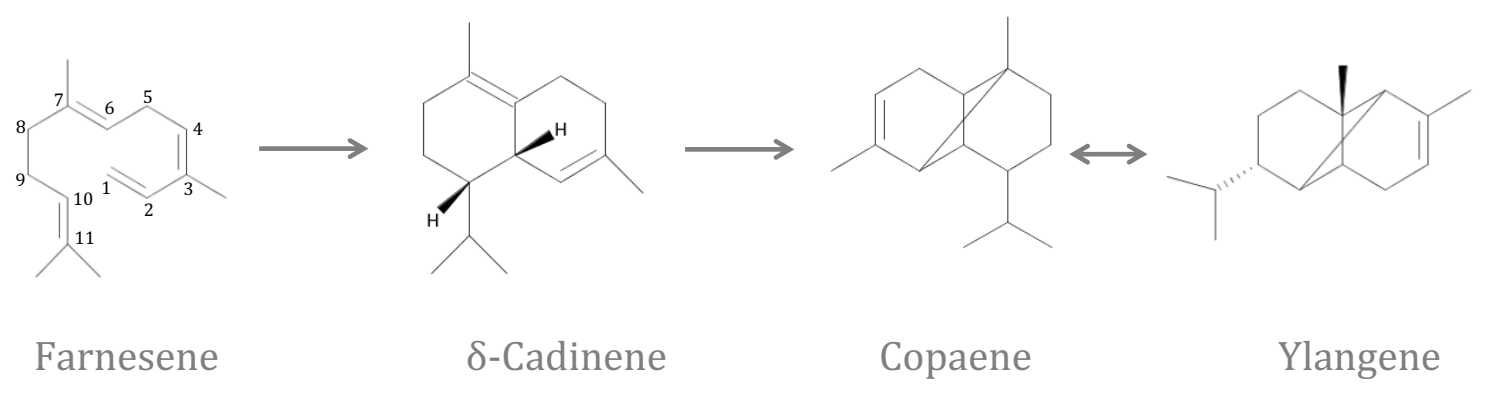

Figure 29: Ring closures of C-1 and C-6 plus C-5 and C-10 leading to the category of sesquiterpenes to include copaene and $\delta$-cadinene.

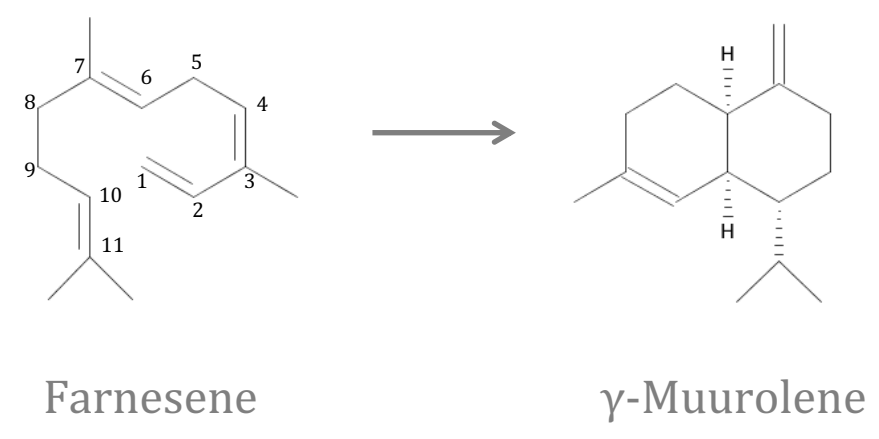

Figure 30: Ring closures of C-1 and C-6 plus C-5 and C-10 leading to the category of sesquiterpenes to include $\gamma$-muurolene. 

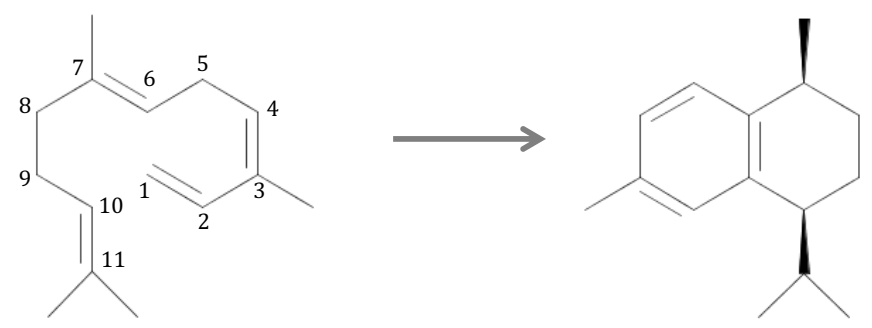

Farnesene

\section{Calamenene}

Figure 31: Ring closures of C-1 and C-6 plus C-5 and C-10 leading to the category of sesquiterpenes to include calamenene.

Ring closures of $\mathrm{C}-1$ and $\mathrm{C}-10$ lead to $\delta$-elemene, through the class of sesquiterpenes known as germacrenes (Figure 32). Germacrenes are important intermediates of over five hundred sesquiterpenes, and are produced because of their antiinflammatory, antimicrobrial and antibacterial, and antifeedant activities. $\delta$-Elemene is formed through Cope rearrangement of the germacrene molecule ${ }^{148} . \delta$-Elemene was present in $93 \%$ of infected samples and $100 \%$ of uninfected samples.

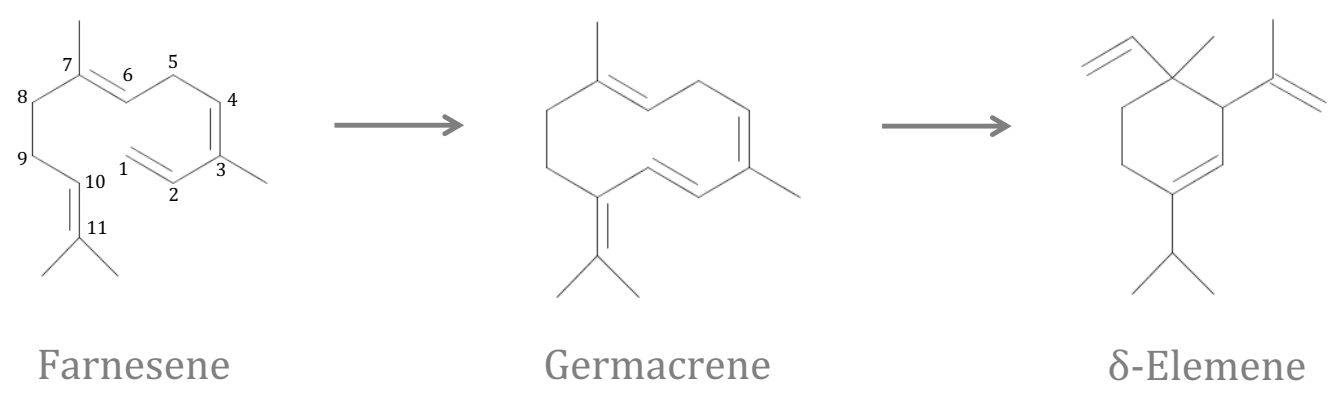

Figure 32: Ring closures of $\mathrm{C}-1$ and $\mathrm{C}-10$ leading to the germacrene category of sesquiterpenes.

Ring closures of C-1 and C-11 lead to caryophyllene oxide (Figure 33). Caryophyllene is formed through humulene by the mentioned ring closures plus a bond 
between $\mathrm{C}-2$ and $\mathrm{C}-10$ of the humulene molecule to form a cyclobutane ring. Caryophyllene oxide was present in $100 \%$ of infected samples, but only $33 \%$ of uninfected samples. Most caryophyllene in infected samples forms the oxide, since caryophyllene was present in only $40 \%$ of infected samples. Caryophyllene oxide has been described as having significant fungicidal effects ${ }^{149}$. The presence of caryophyllene oxide in infected samples is possibly the trees' response to laurel wilt disease, and should be studied further.

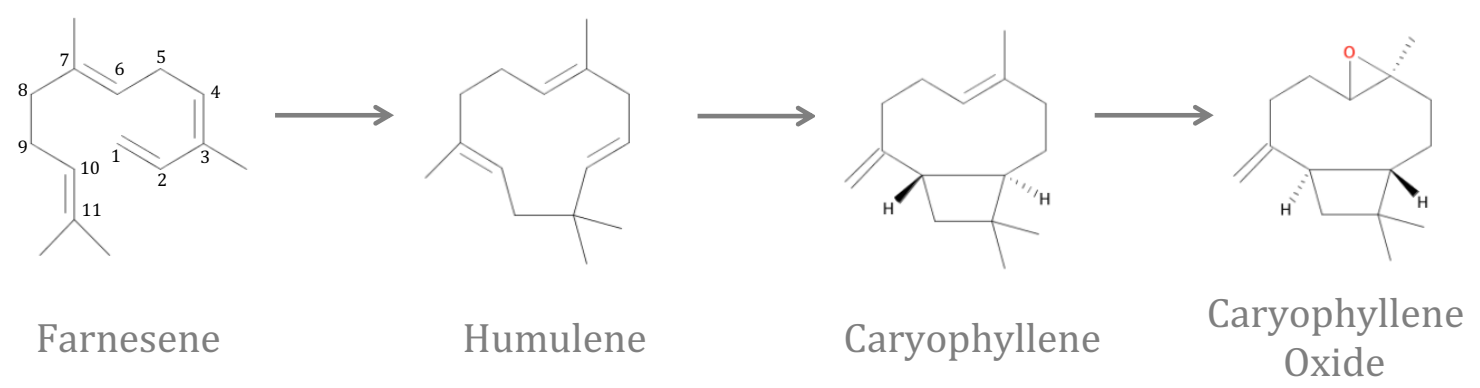

Figure 33: Ring closures of C-1 and C-11 leading to the humulene category of sesquiterpenes.

In another brief experiment, samples of infected avocado trees that were in "early" and "late" stages of infection (visually determined based on amount of wilting) were collected from avocado groves in South Florida using the methods from section 4.2.2. The identified compounds are given in Figure 34. Of the five compounds seen in the late infection sample, three were unique to that stage of decay, the largest contributor being longipinene. Longipinene is formed from the ring closure of C-1 and C-6 plus C-1 and C11 in the farnesene molecule, a ring closure not seen in any other samples of avocado. Longipinene, in addition to the other differences in odor profile, show that as the disease progresses and the tree begins to die, it alters the processes it uses to produce energy and 
begins to produce much less odor. In fact, the overall odor abundance of the early stage of infection was approximately 4.5 times that of the late stage sample.

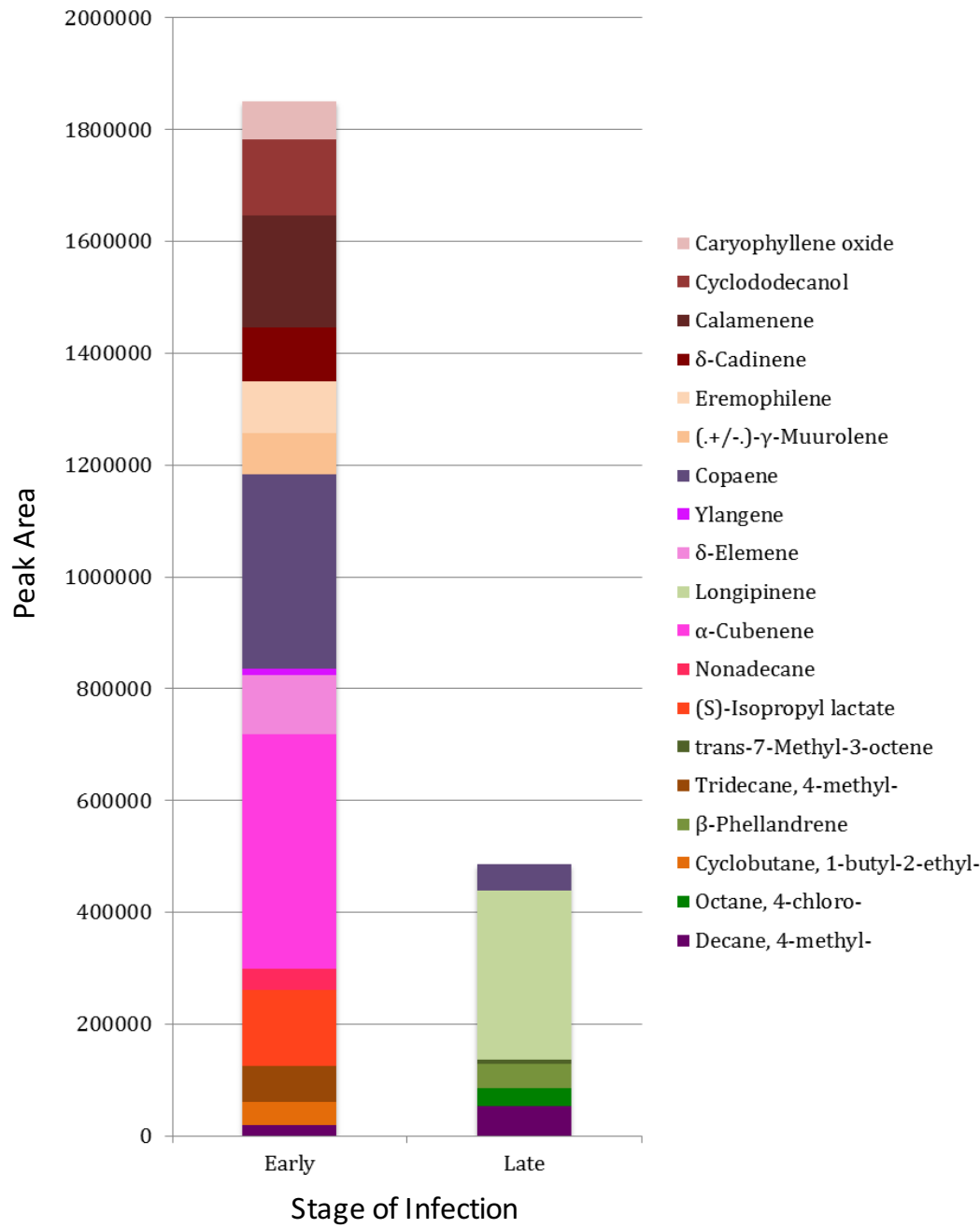

Figure 34: Odor profiles of compounds identified in early and late stages of laurel wilt infection in avocado trees. Purple shades indicate compounds present in both odor profiles. Green shades indicate compounds present only in the odor profile of late stage infection. Warm shades (red, orange, and pink) indicate compounds present only in the odor profile of early stage infection. 
5.2.3. Comparing Volatile Organic Compounds from Infected Avocado Trees to Those of Healthy Avocado Trees

VOCs were detected in the headspace above Lula variety uninfected $(n=9)$ avocado trees using HS-SPME-GC-MS ${ }^{24}$, and subsequently compared to the results of Section 5.2.2. Eight compounds present in at least $80 \%$ of samples were positively identified and confirmed using either standard mixtures or a mass spectral library (NIST 2000 MS Search 2.0 and AMDIS, Gaithersburg, MD). These compounds included $\delta$-elemene, copaene, $\gamma$ muurolene, $\delta$-cadinene, calamenene, $\alpha$-selinene, $\alpha$-cubenene, and (-)-alloaromadendrene (Table 13). Five of the compounds identified were also in infected samples, and three were unique to uninfected samples ( $\alpha$-selinene, $\alpha$-cubenene, and (-)-alloaromadendrene). Of the six compounds found in infected samples, only one was not identified in the uninfected samples, caryophyllene oxide.

Ring closures of C-1 and C-10 plus C-2 and C-7 lead to $\alpha$-selinene (Figure 35). $\alpha$ Selinene was present in $60 \%$ of infected samples and $78 \%$ of uninfected samples. While it was considered not present in infected samples, a methyl shift from $\mathrm{C}-10$ to $\mathrm{C}-14$ forms eremophilene, which was present in $67 \%$ of infected samples yet $0 \%$ of uninfected samples. Much like $\gamma$-muurolene and muurolene, it is probable that eremophilene is formed by the infected trees' increased energy consumed in fighting the disease. 


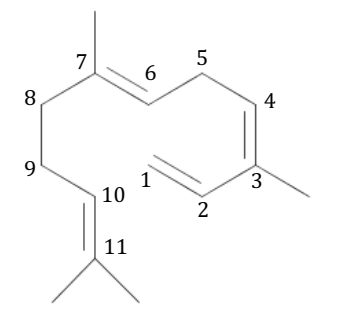

Farnesene

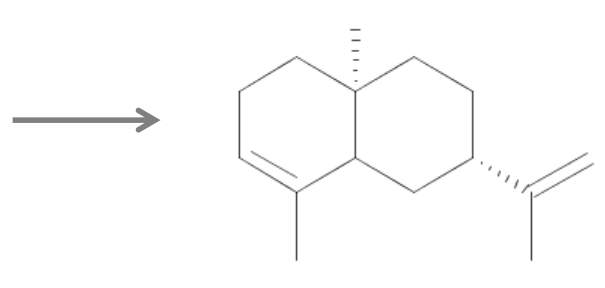

$\alpha$-Selinene

Figure 35: Ring closures of C-1 and C-10 plus C-2 and C-7 leading to the category of sesquiterpenes to include $\alpha$-selinene.

Ring closures of C-1 and C-10 plus C-2 and C-6 lead to $\alpha$-cubenene (Figure 36) and (-)-alloaromadendrene (Figure 37). $\alpha$-Cubenene and (-)-alloaromadendrene are part of the pseudoguaiene sub-category of sesquiterpenes, which results from a methyl shift from C4 to $\mathrm{C}-5$ on the guajene molecule. $\alpha$-Cubenene contains a bond between $\mathrm{C}-1$ and $\mathrm{C}-6$, while (-)-alloaromadendrene contains a bond between C-6 and C-11. Certain categories of sesquiterpenes are increased or decreased in abundance when a tree is suffering from laurel wilt disease. Guaienes decreased in infected trees by about $66 \%$. However, every other category was increased: germacrenes; cadinenes, muurolenes, \& calamenenes; humulenes; and eremophilenes. While the role of guaienes in plant metabolism is unknown ${ }^{150}$, it is notable that it is the only category of sesquiterpene that does not appear to be involved in antifungal activity. 


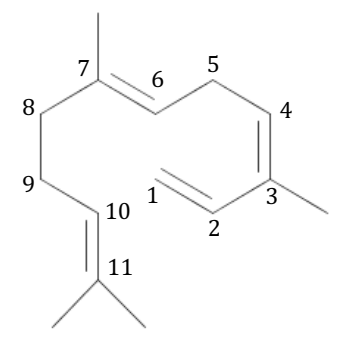

Farnesene

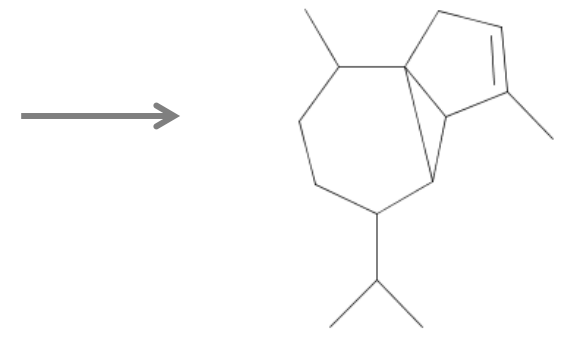

$\alpha$-Cubenene

Figure 36: Ring closures of C-1 and C-10 plus C-2 and C-6 leading to the pseudoguaiene sub-category of sesquiterpenes to include $\alpha$-cubenene.

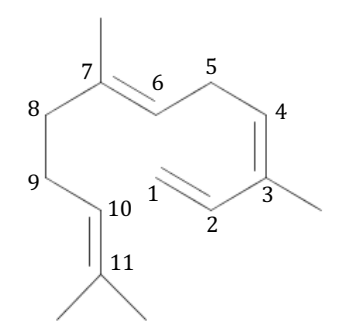

Farnesene

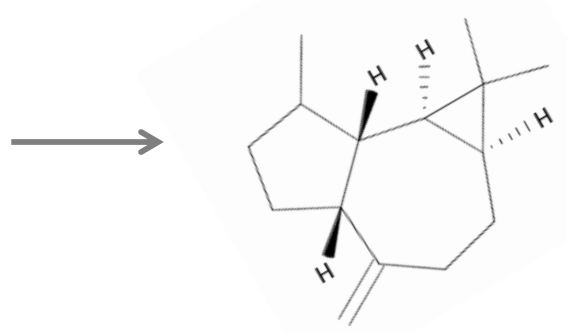

$(-)$-Alloaromadendrene

Figure 37: Ring closures of C-1 and C-10 plus C-2 and C-6 leading to the pseudoguaiene sub-category of sesquiterpenes to include (-)-alloaromadendrene.

Because of the variability of individuals in P. americana, several studies have examined the VOCs produced by healthy avocado trees to try and identify commonalities ${ }^{18-}$ ${ }^{20}$. Niogret et al. 2013a identified six sesquiterpenes common in trunk samples, noting differences among three varieties of avocado trees ${ }^{19}$. Niogret et al. $2013 \mathrm{~b}$ detected 20 sesquiterpenes in trunk samples of avocado trees ${ }^{20}$. Due to proximo-distal gradients, $\alpha$ copaene is higher in the trunk than in other areas of the trees and the trunk contains fewer monoterpenes ${ }^{20}$. Niogret et al. 2011 identified eleven terpenoids, seven of which were 
sesquiterpenes ${ }^{18}$. These studies are useful in establishing the variability of VOCs in the headspace of avocado trees and are listed in Table 1.

The current studies identified nine sesquiterpenes in Lula variety avocado trees, adding to the library of literature (Table 1) and confirming that there is variation between individual trees, but that certain commonalities can also be established. The current research was the first study to identify sesquiterpenes in infected avocado trees. Additionally, only five of the nine compounds identified here were also identified in previous studies. The compound caryophyllene oxide produced by the humulene backbone was identified as being unique to the infected trees using the parameters described in the methods section, and was not previously identified in avocado trees. Additionally, $\gamma-$ muurolene, calamenene, and $\alpha$-selinene were not previously identified (though muurolene was identified $\left.{ }^{20}\right)$.

For the five terpene backbones identified, compounds belonging to the groups eudesmene and guaiene decreased in infected trees, suggesting that they may not be necessary for fighting $R$. lauricola. On the other hand, compounds belonging to the group humulene were increased in infected trees, suggesting that they may be involved in fighting the pathogen. Compounds belonging to the groups germacrene and cadinene, muurolene, \& calamenene existed in both uninfected and infected trees, which was expected because copaene, produced from cadinene, is the main attractant for X. glabratus.

In another brief experiment, uninfected and infected samples were compared to an avocado tree that had been subjected to a lightning strike. Commercial avocado trees are subject to many hazards beside laurel wilt disease, such as fruit stress, drought, or lightning 
strikes. To compare the VOCs above trees suffering different types of stress, samples were taken from an avocado tree injured by a lightning strike using the methods from Section 4.2.2. The compounds are listed in Table 14 and the odor profiles are compared in Figure 38. The VOCs produced by the lightning-injured tree were more similar to the uninfected samples, as seen in the PCA in Figure 39. In the figure, the infected trees cluster more closely than the uninfected or lightning-strike samples, indicating that trees fighting an infection will produce a different set of odors than a tree injured or stressed by another cause, probably due to different reaction mechanisms in

Table 14: Compounds present in avocado tree injured by lightning.

\begin{tabular}{|c|c|c|}
\hline & CAS No. & Molecule \\
\hline 1 & $20307-84-0$ & $\delta$-Eiemene \\
\hline 2 & $3856-25-5$ & Copaene \\
\hline 3 & $30021-74-0$ & ү-Muurolene \\
\hline 4 & $483-76-1$ & $\delta$-Cadinene \\
\hline 5 & $483-77-2$ & Calamenene \\
\hline 6 & $473-13-2$ & $\alpha$-Selinene \\
\hline 7 & $17699-14-8$ & $\alpha$-Cubenene \\
\hline 8 & $5989-54-8$ & Limonene \\
\hline 9 & $624-42-0$ & 3-Heptanone, 6-methyl- \\
\hline 10 & $30824-67-0$ & -Elemene \\
\hline 11 & & Aristolene \\
\hline 12 & & trans- $\alpha$-Bergamotene \\
\hline 13 & $515-13-9$ & $\beta$-Elemene \\
\hline 14 & $87-44-5$ & Caryophyllene \\
\hline 15 & $3650-28-0$ & (+)-Sativene \\
\hline 16 & 5951-61-1 & Cadinene \\
\hline 17 & 10219-75-7 & Eremophilene \\
\hline 18 & $22469-52-9$ & Cyclosativene \\
\hline 19 & $14912-44-8$ & Ylangene \\
\hline 20 & $6813-21-4$ & 3,7(11)-Selinadiene \\
\hline 21 & $16982-00-6$ & Cuparene \\
\hline
\end{tabular}




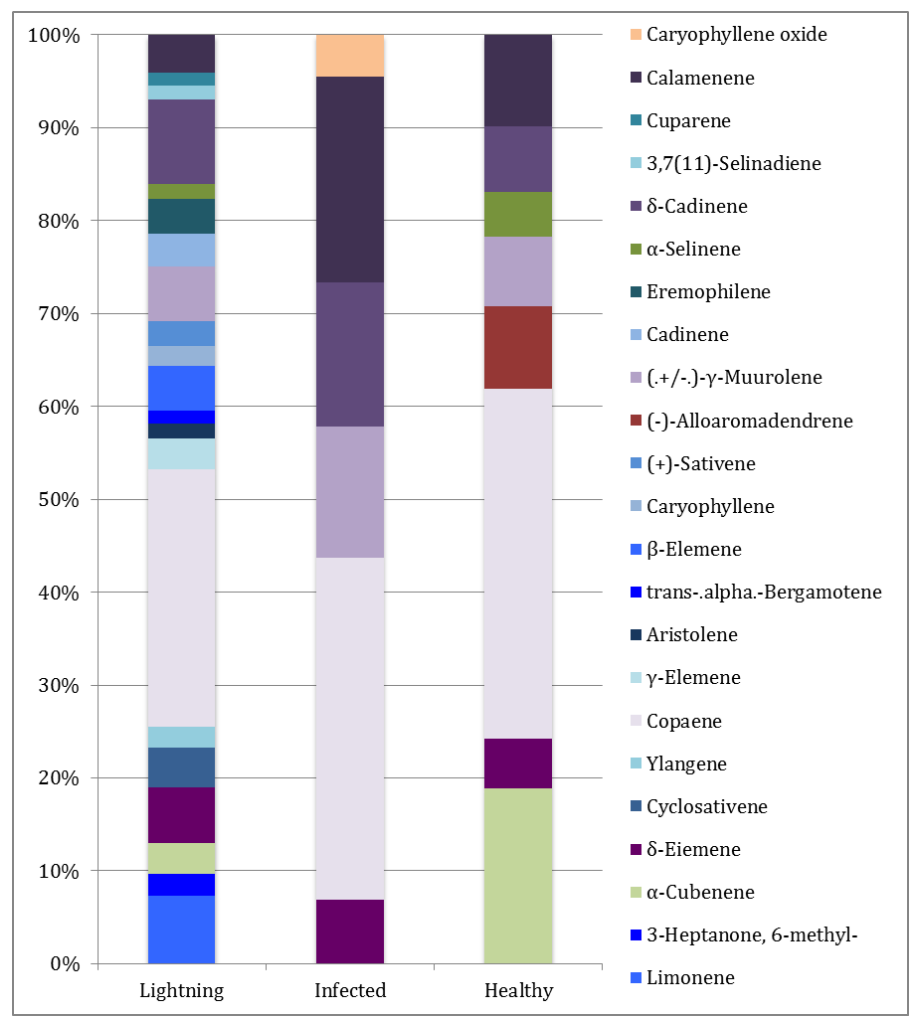

Figure 38: Odor profiles of avocado trees struck by lightning, infected with laurel wilt, and uninfected (neither struck by lightning nor infected with laurel wilt). Purple shades indicate compounds present in all three odor profiles. Green shades indicate compounds present in just those odor profiles of lightning struck and healthy trees. Blue shades indicate compounds present in only the lightning struck tree odor profile.

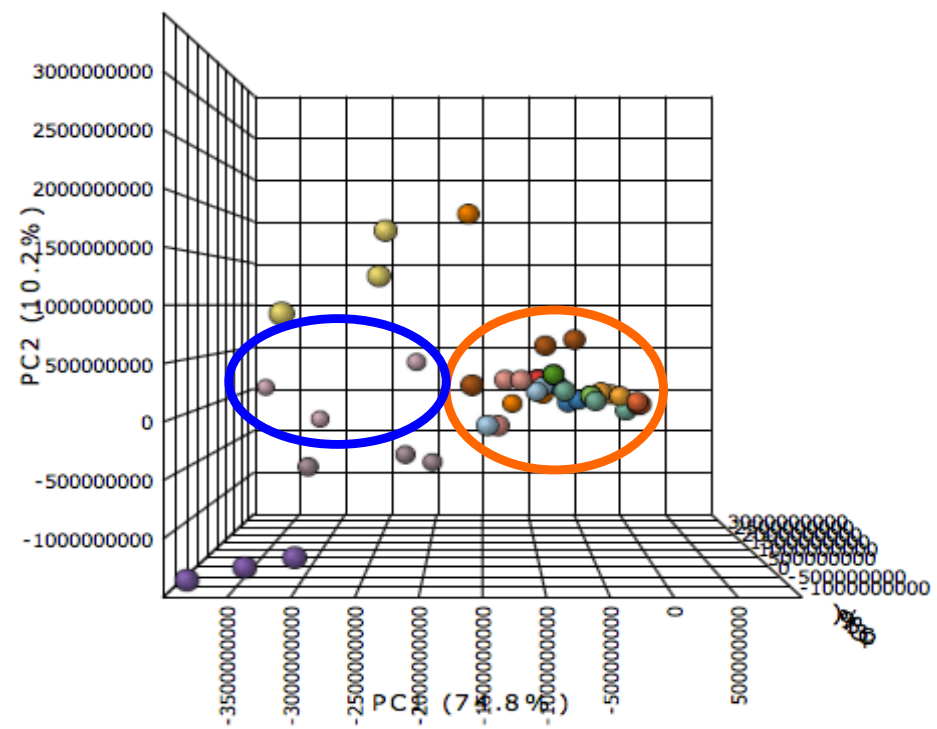

Figure 39: PCA of infected avocado tree odors (orange circle), lightning struck avocado tree (blue circle), and healthy avocado tree odors. 
each case. Markedly, caryophyllene oxide was not produced by the lightning struck tree. As has been noted, caryophyllene oxide compound is produced as an antifungal aid ${ }^{149}$, so it was expected to not be produced when the tree is stressed due to a non-fungal stressor, like lightning. Additionally, (-)-alloaromadendrene was only produced by the healthy tree. The results support the above conclusion that the ring closures forming the guaiene category of sesquiterpenes are not necessary to correct or fight injury.

Further, laurel wilt disease affects all members of the Lauraceae, and has caused the deaths of over 300 million wild trees ${ }^{30,46,47}$. Samples were taken from an infected wild swampbay tree and an uninfected wild swampbay tree using the methods from Section 4.2.2. While swampbays and avocados have different odor profiles, they dis share some similarities, as shown in the PCA in Figure 40. Infected trees of both species group closely together. The compounds identified in healthy and infected swampbay trees are given in Table 15. There were two compounds that are in healthy and infected samples of both species of tree: copaene and $\gamma$-muurolene. These are the only two compounds that infected swampbay trees had in common with either infected or healthy avocado trees, and both are produced from the same farnesene ring closure. Notably, copaene is the main attractant of $X$ glabratus, so it is not surprising that both laurel wilt-susceptible species produce it. As can be seen in the odor profiles (Figure 41), avocados and swampbays produce unique VOCs. For example, avocado trees produce $\delta$-elemene and $\delta$-cadinene, while swampbays produce many monoterpenes, such as eucalyptol and limonene. Eucalyptol was recently identified as an enhancement for $X$. glabratus traps ${ }^{151}$. Since it is not seen in avocados, the presence of eucalyptol could help explain why so few of the vectors are identified in avocado groves, and should be further explored in future research. 


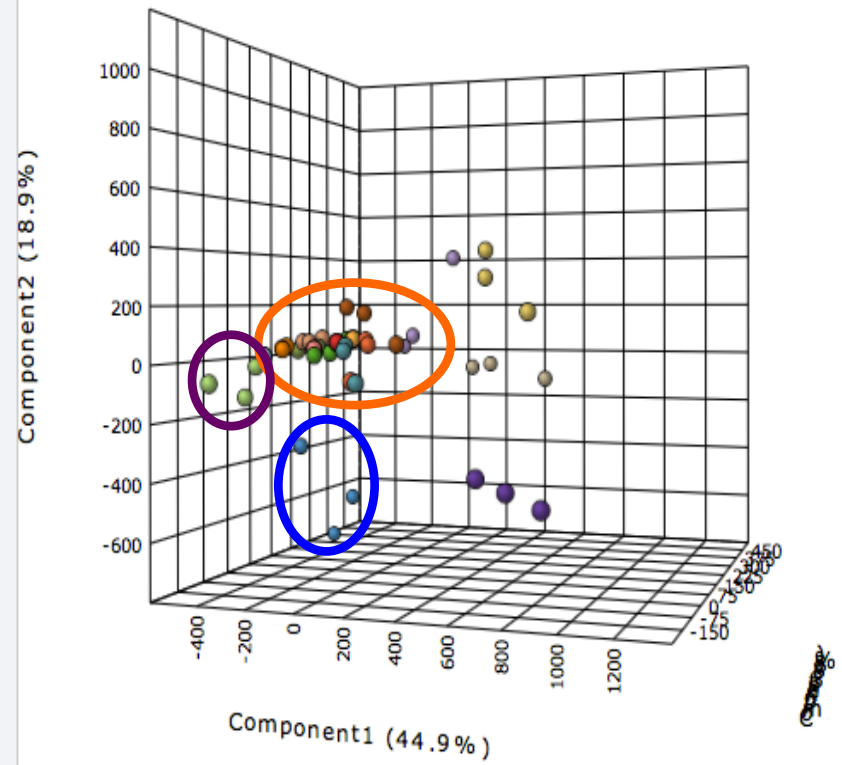

Figure 40: PCA of infected avocado tree odors (orange circle), infected swampbay tree odors (purple circle), healthy swampbay tree odors (blue circle), and healthy avocado tree odors.

Table 15: Compounds present in healthy and infected wild swampbay trees.

\begin{tabular}{|c|c|c|c|c|}
\hline & CAS No. & Compound & Healthy & Infected \\
\hline 1 & $18172-67-3$ & (-)- $\beta$-Pinene & $\checkmark$ & $x$ \\
\hline 2 & $555-10-2$ & $\beta$-Phellandrene & $\checkmark$ & $x$ \\
\hline 3 & $3387-41-5$ & Sabinene & $\checkmark$ & $x$ \\
\hline 4 & $99-87-6$ & p-Cimene & $\checkmark$ & $x$ \\
\hline 5 & $17699-14-8$ & $\alpha$-Cubenene & $\checkmark$ & $x$ \\
\hline 6 & $464-49-3$ & (+)-Camphor & $\checkmark$ & $x$ \\
\hline 7 & $17334-55-3$ & $\beta$-Gurjenene & $\checkmark$ & $x$ \\
\hline 8 & $523-47-7$ & $\beta$-Cadinene & $\checkmark$ & $x$ \\
\hline 9 & $483-77-2$ & Calamenene & $\checkmark$ & $x$ \\
\hline 10 & $5989-54-8$ & Limonene & $\checkmark$ & $\checkmark$ \\
\hline 11 & $470-82-6$ & Eucalyptol & $\checkmark$ & $\checkmark$ \\
\hline 12 & $3856-25-5$ & Copaene & $\checkmark$ & $\checkmark$ \\
\hline 13 & 17699-05-7 & $\alpha$-Bergamotene & $\checkmark$ & $\checkmark$ \\
\hline 14 & $30021-74-0$ & ү-Muurolene & $\checkmark$ & $\checkmark$ \\
\hline 15 & $106-68-3$ & 3-Octanone & $x$ & $\checkmark$ \\
\hline 16 & 56684-96-9 & $\beta$-Neoclovene & $x$ & $\checkmark$ \\
\hline 17 & $3391-86-4$ & 1-Octen-3-ol & $x$ & $\checkmark$ \\
\hline 18 & $3691-12-1$ & $\alpha$-Guajene & $x$ & $\checkmark$ \\
\hline 19 & $1135-66-6$ & Isolongipholene & $x$ & $\checkmark$ \\
\hline 20 & $2460-77-7$ & 2,5-di-tert-Butyl-1, 4-benzoquinone & $x$ & $\checkmark$ \\
\hline
\end{tabular}




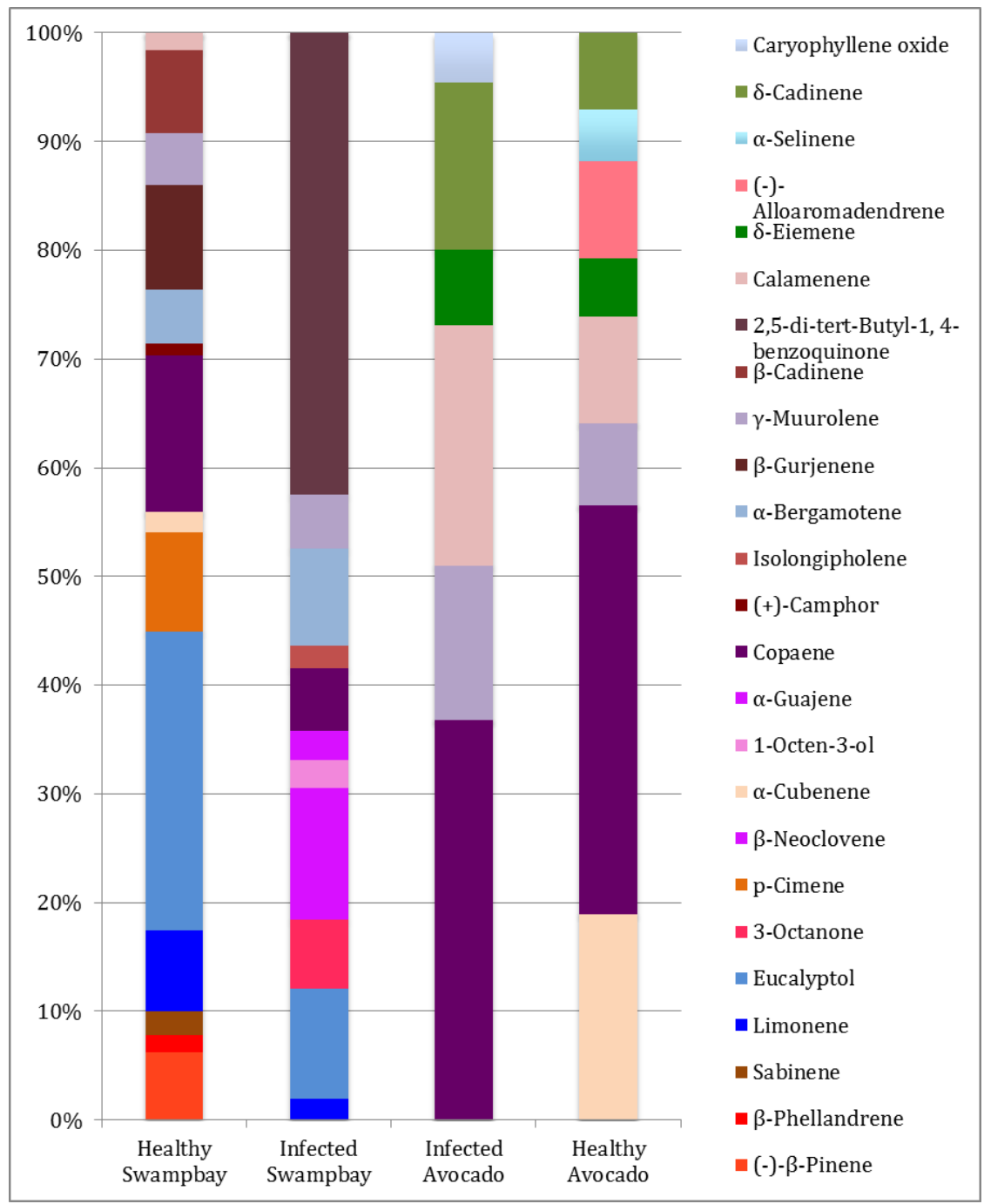

Figure 41: Odor profiles of healthy and infected swampbay trees, and healthy and infected avocado trees. Purple shades indicate compounds present in all four odor profiles. Blue shades indicate compounds present in both healthy and infected swampbay odor profiles, while green shades indicate compounds present in both healthy and infected avocado odor profiles. Warm shades (red, orange, and pink) indicate all other compounds.

The results of Task 2 presented in the current section (Section 5.2) reveal the biological dynamicity of the complicated systems involved in the laurel wilt disease. First, the VOCs of $R$. lauricola were examined. Then, the VOCs of infected adult avocado trees and uninfected adult avocado trees were evaluated. Brief studies of varying stages of infection, lightning-strike injuries, and wild healthy and infected swampbay trees were also 
performed. As can be seen by the results, the volatiles of each of these systems is extremely variable and complex. The knowledge increased understanding of the systems interactions and responses in nature, which ultimately will help in controlling the current spread of disease and preventing further expansion. Future research should focus on continuing to expand the knowledge of VOC production by avocado trees injured through other common factors such as drought, fruit stress, and other pathogens by identifying the VOCs and characterizing their production pathways. Additionally, the VOC production of healthy and infected trees from all other laurel wilt-susceptible species should be studied.

5.3. Task 3: Assessment of a Controlled Odor Mimic Permeation System for the Detection of the Laurel Wilt Disease by Canines

In addition to characterizing the volatile fungal-plant interactions, a goal of the dissertation was to improve canine training for biological targets like the laurel wilt disease. Because only two of the compounds identified in avocado trees are commercially available, the following section (Section 5.3) explored a new method of canine training aid creation for such intricate systems. The purpose of Task 3 was two-fold. First, it established and validated a non-biological canine detection training aid and method of active odor identification (Section 5.3.1). Second, it characterized current training aids, COMPS containing infected wood, through quantitative analyses. Results were used to establish properties such as shelf-life and permeation rate (Section 5.3.2). The information gained provided a better understanding of and improved methods for canine detection of and training for live target substances. 


\subsubsection{Development of the Column Vent Method for Active Odor Identification}

A current challenge in environmental canine detection is the lack of available training aids containing mimic or pseudo odors of the target odorant ${ }^{96}$. Suitable mimic or pseudo training materials often take a significant amount of time and money to develop as the process of identifying the correct active odorants and their respective ratios can be highly time-consuming ${ }^{97,101-104}$. Due to the dedication of time and money, mimics or pseudo training aids may never be developed, but the column vent method presented here and the results described show how the combination of the analytical HS-SPME-GC-MS method with detection canines can allow for more rapid active odor identification and production of safe training aids. The process involves analytical identification of the compounds present followed by canine identification of chromatographic fractions containing the active odor chemicals. Since only two of the nine compounds identified in Sections 5.2.2 and 5.2.3 are commercially available as standards, the column vent fractions were designed to allow canines to select chromatographic areas of interest from samples of infected avocado trees, without having to obtain all compounds of interest commercially. The chromatograms of the fractions presented to the canines are displayed in Figure 43 (refer to Table 8). Figure 42 gives the organizational design of the project. These results are published in Simon et al. $(2017)^{24}$. 


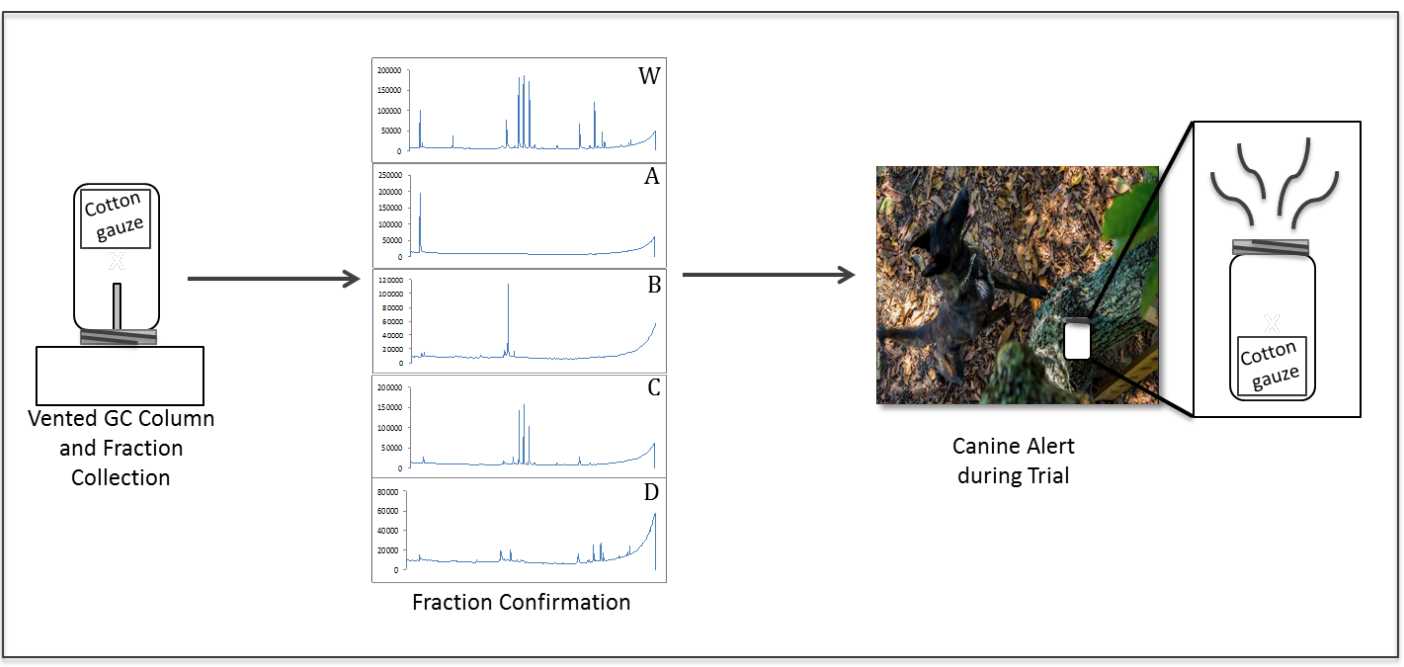

Figure 42: Flow chart of the organizational design of the column vent method. Fractions are collected on cotton gauze from GC column vented to the atmosphere. Then, the fractions are confirmed through GC-MS. Finally, the fractions are presented to canines during field trials.

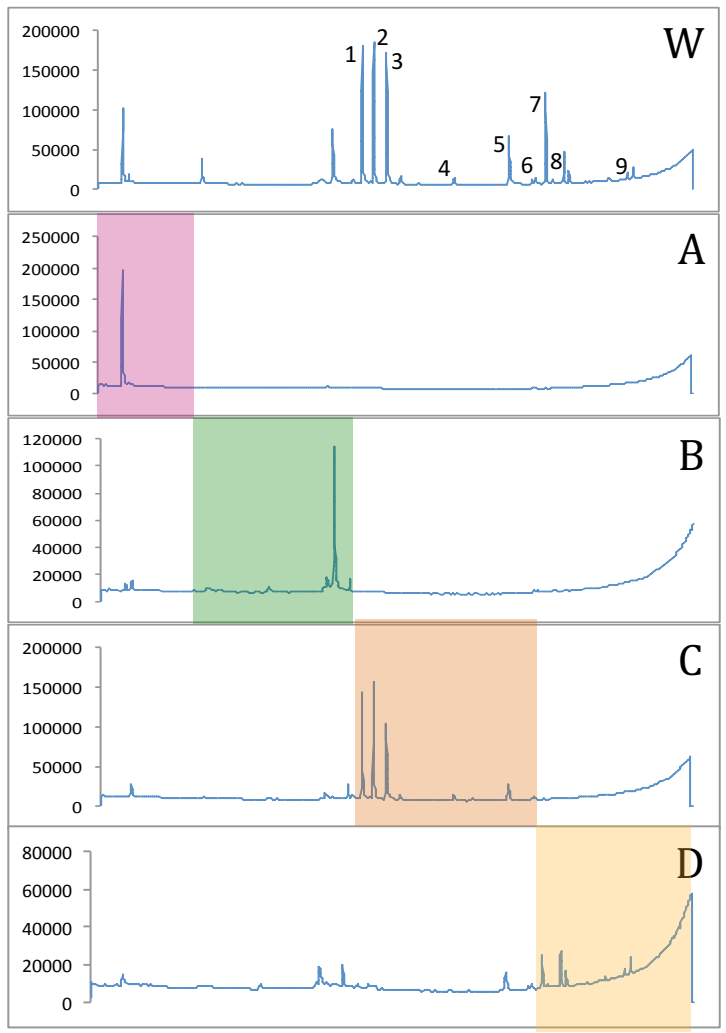

Figure 43: Chromatograms of fractions A, B, C, D, and W presented to canines. On the chromatograms representing fractions $A, B, C$, and $D$, the compounds contained in each vial are highlighted. Compounds are labeled in fraction W: 1) $\alpha$-cubenene, 2) $\delta$-elemene, 3) copaene, 4) (-)-alloaromadendrene, 5) $\gamma$-muurolene, 6) $\alpha$ selinene, 7) $\delta$-cadinene, 8) calamenene, and 9) caryophyllene oxide. 
The results of the canine trials are presented in Table 16 and the trial designs are presented in Figure 15 (Section 4.3.1.2). Canine trials included in the results are trials where Canines 1 and 2 alerted to the positive control (COMPS) and wind speed was below 15mph (24kph). In the data included below (for 6 total canine trial runs), canines alerted $100.0 \%$ to the COMPS with infected wood and $0.0 \%$ to the blanks, which constituted both of the controls. The alert rate for fraction A was $50.0 \%$ and the alert rate for fraction B was $33.3 \%$, while the alert rate for fractions $\mathrm{C}$ and $\mathrm{D}$ were both $0.0 \%$.

Table 16: Canine trial results presented as alert rate (\%) and no alert rate (\%).

\begin{tabular}{|c|r|r|}
\hline Training Aid & Alert Rate (\%) & \multicolumn{2}{|c|}{$\begin{array}{l}\text { No Alert } \\
\text { Rate (\%) }\end{array}$} \\
\hline Blank & 0.0 & 100.0 \\
\hline COMPS & 100.0 & 0.0 \\
\hline W & 100.0 & 0.0 \\
\hline A & 50.0 & 50.0 \\
\hline B & 33.3 & 66.7 \\
\hline C & 0.0 & 100.0 \\
\hline D & 0.0 & 100.0 \\
\hline
\end{tabular}

The canines alerted to fractions $\mathrm{A}$ and $\mathrm{B}$, which contain the most volatile compounds, demonstrating that the compounds of interest elute in the first half of the chromatogram. Using the information, the compounds from fractions A and B may be used to create training aids that mimic the active odors present in the headspace of infected trees or could be combined in future studies. The multiple training aids can be used to present various ratios of the VOCs from fractions A and B to address the variability of 
individual tree samples. The canines alerted $100 \%$ to fraction $\mathrm{W}$, which represents the complete odor profile, confirming that the active odors of the infected samples' headspace were in fact extracted and collected using the column vent method. The high percent loss for the second half of the chromatogram was determined to not be a factor because the $100 \%$ alert rate to fraction $\mathrm{W}$ confirmed that all active odors were extracted (Table 9).

The study produced a new method for extracting and creating canine training aids. Chemical analysis was performed using HS-SPME-GC-MS. Canine analysis was performed by using the column vent method described to collect odor fractions presented to the canines in a short series of field experiments. The approach allowed the canines to detect the active odors of the laurel wilt disease based on chromatographic areas of interest, which can aid the development of a training method that would not require the use of live cultures, and presents a method that could reduce the dependence on live training aids.

The method helps to overcome the challenge presented by the availability of environmental training aids. Individuals of a tree species will have different terpenoid mixtures based on genetic, developmental, and environmental factors, though commonalities within species are also prevalent ${ }^{2,20,144-146}$. To overcome the variability distinguishing VOCs of individuals of the species, it is suggested that multiple training aids be used. The approach was suggested by Macias \& Furton (2011) regarding the variability in the headspace of MDMA samples ${ }^{152}$. In instances where the VOCs present and the threshold of the active odors differ, it can be necessary to use more than one training aid to provide optimal canine training. 
Analytical instrumentation and canine detection can act as complimentary techniques and result in the rapid identification of active odors in the headspace of target substances. The column vent method permitted a faster process for the identification of VOCs, accelerating the creation of mimic training aids. These training aids can then be used as to avoid the risks associated with live training aids, such as rarity of the species, legality of obtaining the species, and methodology of odor or species containment. Additionally, the column vent method can be used to exclude VOCs identified as nonactive components, such as fractions $\mathrm{C}$ and $\mathrm{D}$ in Lula variety infected avocado trees. Future research should test other environmental targets using the column vent method to verify its application across the field.

\subsubsection{Characterization of the Canine Training Aid Used in the Field}

Training aids for laurel wilt detection canines are currently COMPS holding avocado infected wood (Figure 44), as described in Section 4.3.2. The utilization of COMPS is advantageous because it can be created quickly in the field using samples that require no other preparation. Using COMPS prevents mass transport of the infected samples. However, there are also many disadvantages because of unknown variables. For example, the identification of compounds, the concentration of those compounds, and the permeation rate of those compounds all remain undetermined. If these variables are evaluated, then they can inform procedures for use, such as shelf-life and proper storage of the training aids can be understood, increasing the efficacy of the COMPS. 


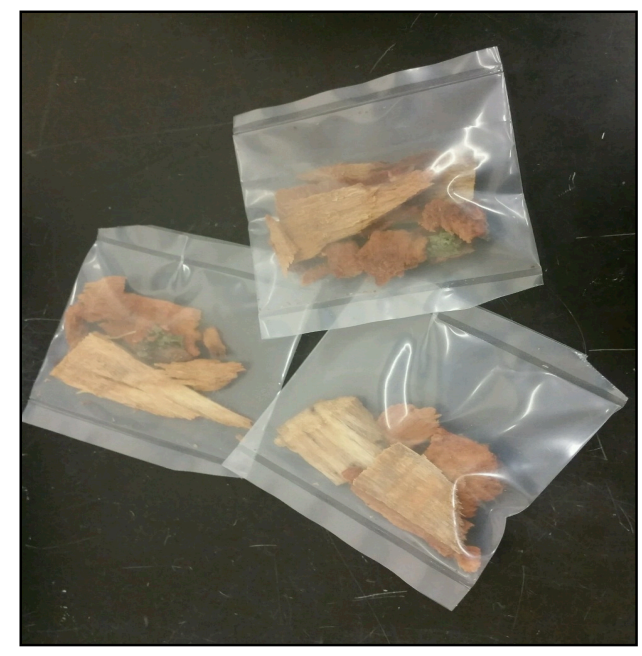

Figure 44: Examples of COMPS for laurel wilt detection canines.

The study was performed in two separate experiments. The first was static sampling of the COMPS, and the second was dynamic sampling. Both sets of experiments were performed using ESIS-SPME. While both infected and uninfected COMPS were sampled for the static tests, no volatiles were recovered from the uninfected samples, so no results are shown for those COMPS. First, peak area was observed (Figure 45) to identify the compounds permeating through the COMPS bag and then determine the abundance of each. Day 3 showed the highest recovery with five compounds: calamenene, $\delta$-cadinene, caryophyllene, copaene, and ylangene. Of these, only caryophyllene and ylangene were not previously identified in infected avocado samples. However, ylangene is an isomer of copaene, as discussed previously, and caryophyllene is an intermediate between humulene and caryophyllene oxide. No compounds were recovered on Day 1, and all compounds were completely dissipated by Day 7. 


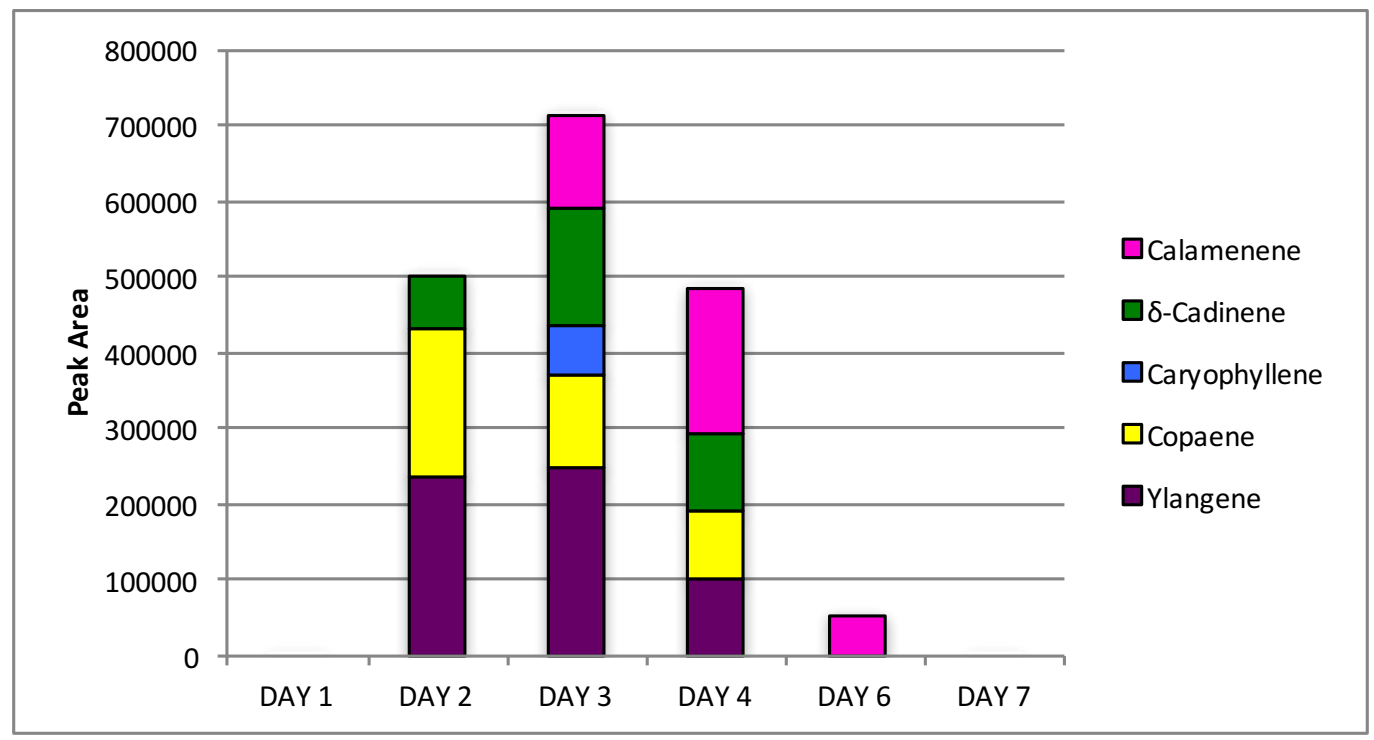

Figure 45: Odor profiles of infected COMPS collected during static sampling by day.

To evaluate permeation rates, Equation 2 was used to calculate $A / E$ ratios for each compound. To show why ESIS-SPME was used, Figure 46 and Figure 47 are given for comparison. Figure 46 shows the abundance of each peak area by day, while Figure 47 gives the $A / E$ ratios for each compound. $A / E$ ratios provide more comparable information. Overall, the abundance of odor decreases overtime, independent of the fluctuation of each compound. By looking at the total odor $A / E$ ratio, the permeation rate was determined to be $3014 A / E$ per day $\left(\mathrm{R}^{2}=0.93357\right)$. Using the information, several recommendations can be made for the utilization of COMPS. The COMPS should be made 24 hours before being used in training scenarios, and discarded after four days. Future research should focus on improving the matrix containing the wood samples to extend shelf-life. 


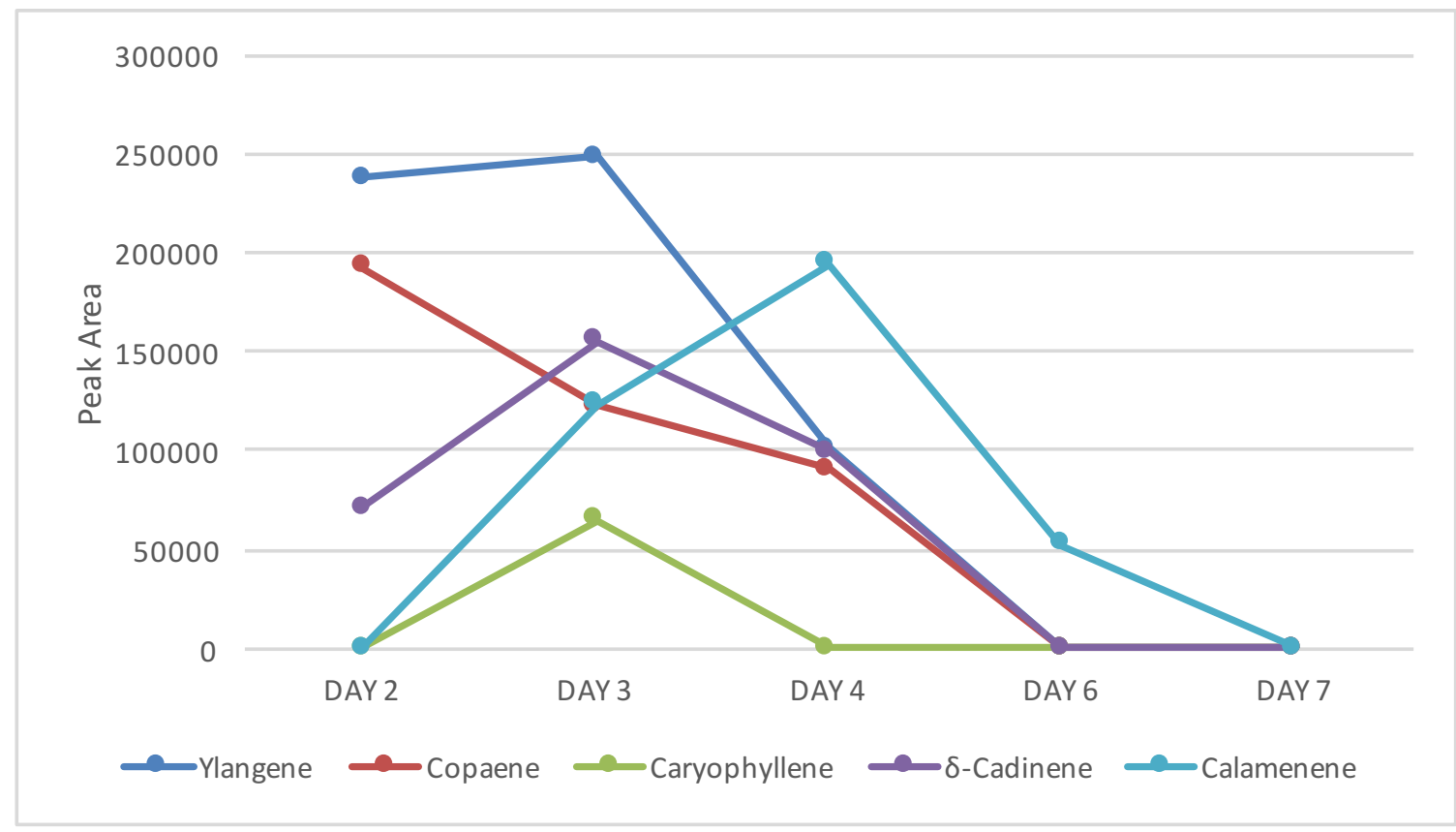

Figure 46: Peak area for each compound recovered from static sampling procedures for infected COMPS.

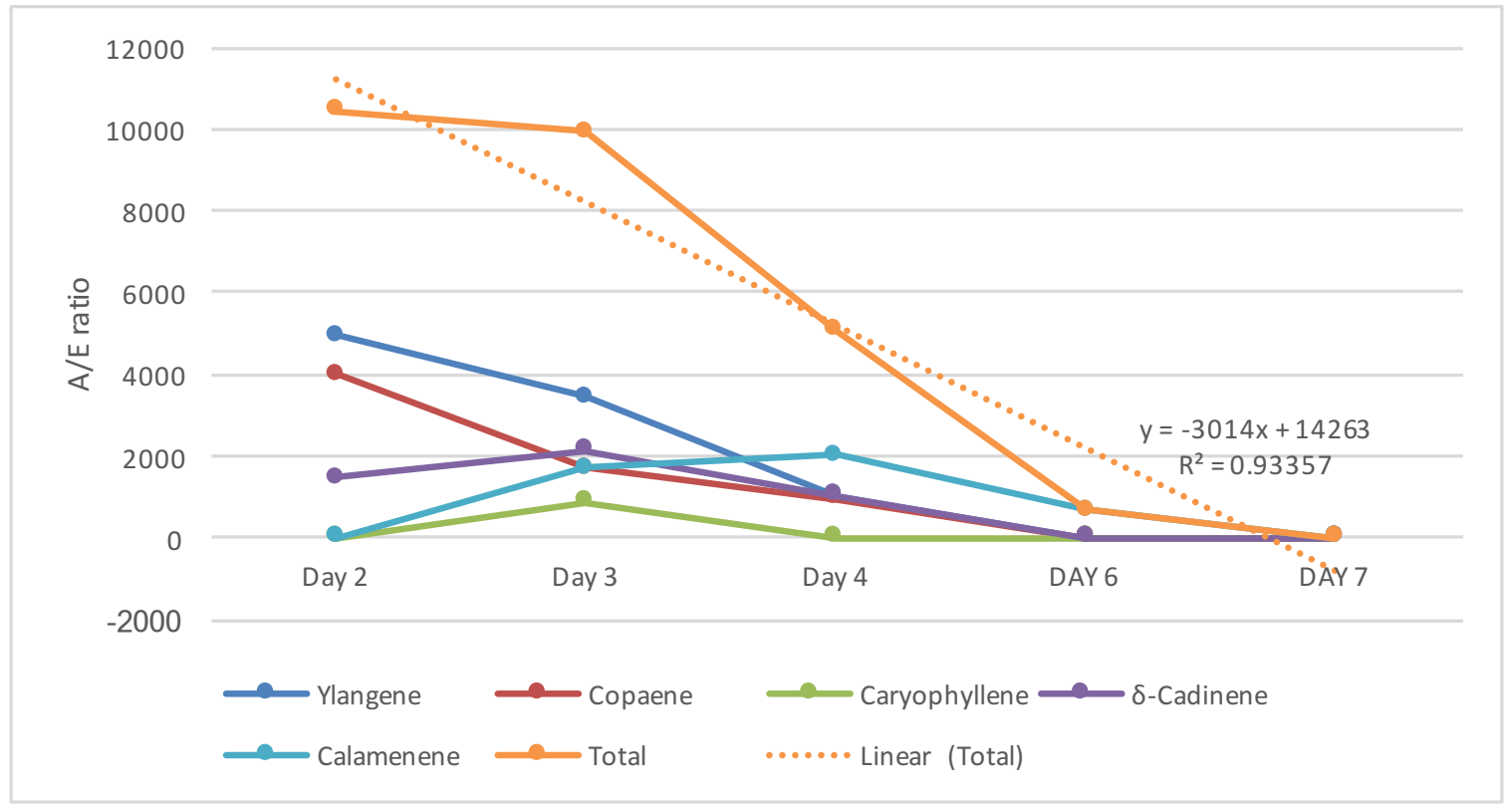

Figure 47: $A / E$ ratios for each compound recovered from static sampling procedures for infected COMPS, plus the total permeation rate. 
The second experiment was performed using the dynamic sampling method. Since no volatiles were recovered for uninfected COMPS during static sampling, no uninfected COMPS were evaluated during dynamic testing. The peak areas of each compound recovered are shown in Figure 48. Four of the five compounds seen in the static testing were also seen here: calamenene, $\delta$-cadinene, copaene, and ylangene. The abundances of each is higher and more consistent over time in comparison to the static sampling. Figure 49 displays the $A / E$ ratios for each compound, plus the total permeation rate $(15703 \mathrm{~A} / \mathrm{E}$ per day with $\mathrm{R}^{2}=0.98864$ ). While the permeation is more consistent, it is also faster. Based on these results, it can be concluded that the current method of storing training aids (contained within non-permeable packaging) is better than storing them in open air. The permeation rate may be higher, but the identity and abundance of compounds is more consistent over the training period. Additionally, while the recommendation remains to create the COMPS 24 hours before training begins, the dynamic sampling shows that shelf-life can be extended to five days simply by changing the storage method. 


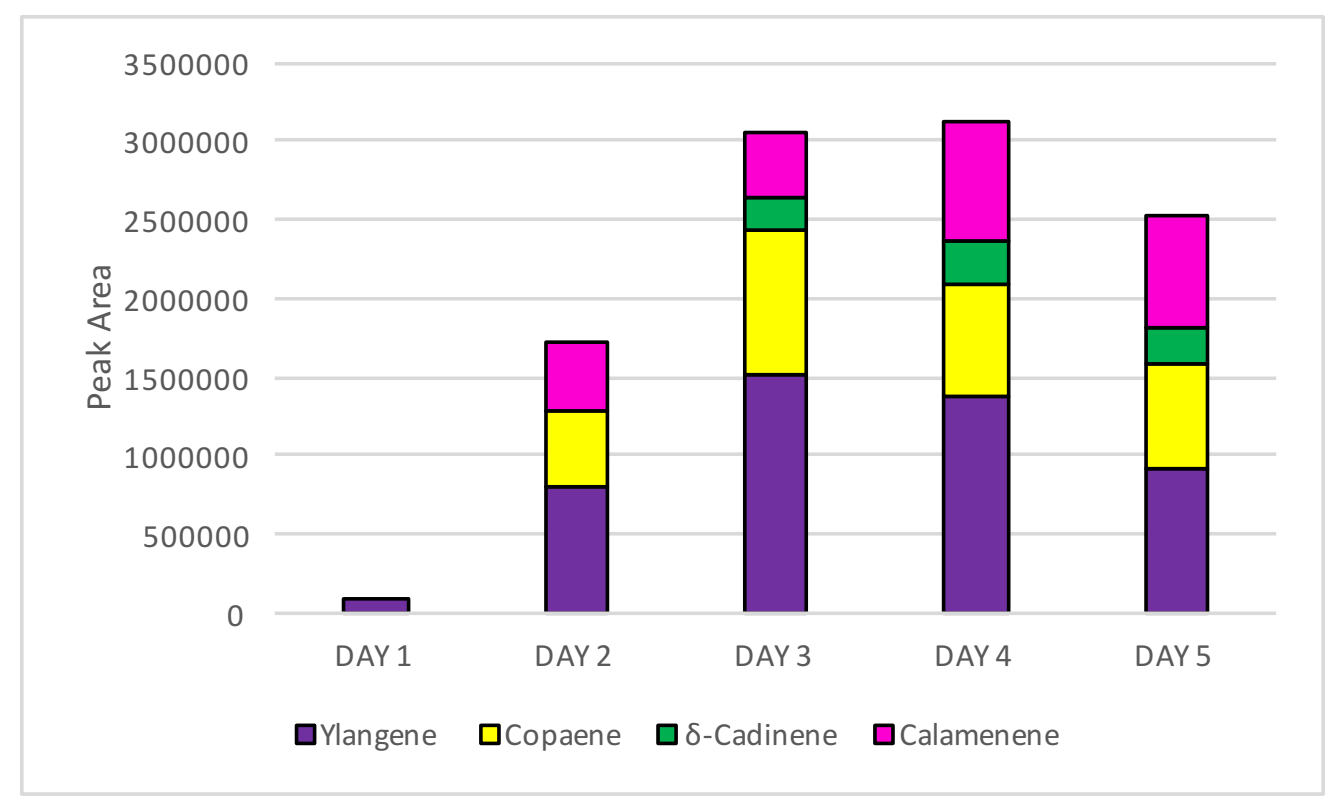

Figure 48: Odor profiles (given in peak area) of infected COMPS collected during dynamic sampling by day.

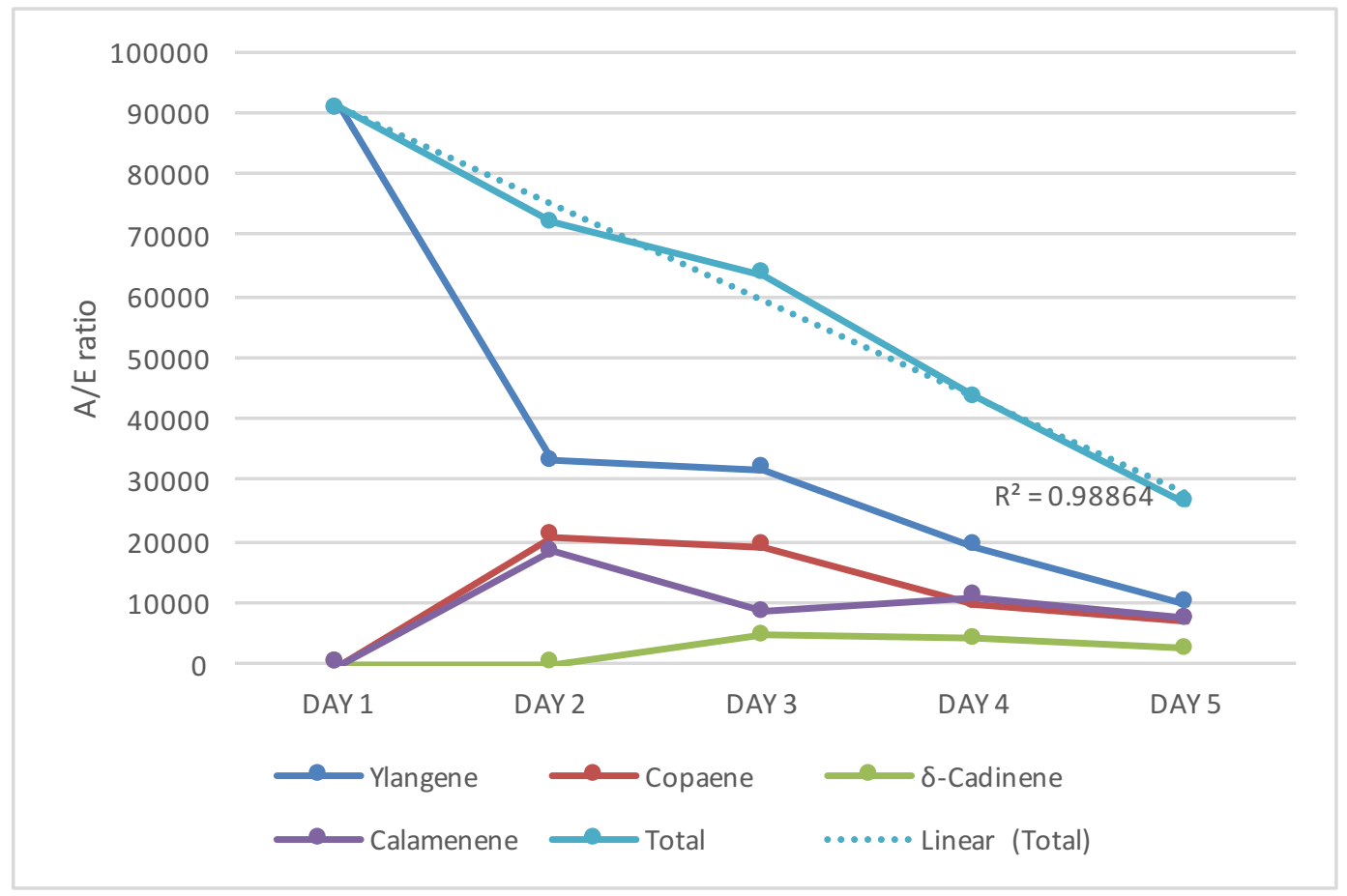

Figure 49: $A / E$ ratios for each compound recovered from dynamic sampling procedures for infected COMPS, plus the total permeation rate. 
Evaluation of COMPS through both static and dynamic sampling methods offers several advantages. Containing the infected samples in LDPE bags contains fungal spores, which reduces the risk of spreading any pest, while allowing for continual release of target odors. Air exchange continues, which allows fungi to continue aerobic processes. COMPS can be made rapidly in the field with little or no preparation of the sample, allowing for rapid training and deployment of canine teams. Knowing the shelf-life of the training aids and permeation rates of the odorants can optimize training efficacy. Even if active odors remain unidentified or unconfirmed, safe and reliable training aids can be created with known permeation rates and shelf-lives. Further, ESIS-SPME-GC-MS was shown to be an effective method for evaluating shelf-life of training aids for biological targets. These COMPS can be rapidly applied to other invasive species, endangered species, biological threats, and hazardous targets.

Because shelf-life and permeation rate have been identified, future research should focus on optimization to extend shelf-life and enhance permeation rate. Extending those parameters would minimize the number of difficult-to-obtain target samples that must be collected to create these COMPS. Consistent and reliable training aids can be provided to train canines to better protect borders and prevent biotic homogenization.

5.4. Task 4: Evaluation of Volatile Organic Compounds of the Laurel Wilt Disease in Avocados Using Greenhouse Experiments

The purpose of Task 4 was to evaluate VOCs of infected avocado trees in a controlled setting. Therefore, young avocado trees of one variety (Lula) were inoculated with suspensions of R. lauricola. Inoculated trees (Section 5.4.1), inoculated trees treated with 
fungicide (Section 5.4.2), and healthy control trees (Sections 5.4.1 and 5.4.2) were each observed for VOC profiles. Treated trees were included because there is anecdotal evidence that canines stop alerting to such trees. Studying the VOCs of these three groups of trees helped identify the active odorants of infected avocado trees and provided information on how avocado trees react to the infection as well as to treatment with propiconazole. VOC observation began before inoculation and followed the initial laurel wilt disease cycle. At the end of each study, a section of every tree was taken for SEM to visualize the presence of tylose in infected trees.

5.4.1. Examining the Volatile Organic Compounds of Avocado Trees Inoculated with Raffaelea lauricola

Ten trees, five inoculated and five control, were observed over a ten week period. By following the VOCs of the trees during the ten week time frame, it was possible to determine if and how inoculation with $R$. lauricola affects odor, and in turn identify possible target odorants for stand-off and canine detection methods. The information can be used to assist in understanding the trees' response to the fungus and in improving disease detection by canines.

Odor profiles for the inoculated trees over the given time frame are displayed in Figure 50 while results of the control trees are given in Figure 51. Inoculated trees showed an immediate response to infection by increasing the amount and variety of VOCs released, peaking at Day 12. Consistent with the results from Section 5.2.2, guaienes ( $\alpha$-cubebene, $\beta$-cubebene, $(+)$-aromadendrene, and alloaromadendrene) were detected less in inoculated trees than in the control trees for most days. Every other category increased: humulenes, 
eudesmenes, germacrenes, and cadinenes, muurolenes, and calamenenes. Humulenes ( $\beta$ caryophyllene and $\alpha$-humulene) had the highest difference, and was not detected in control trees on most days, supporting the proposed idea that humulenes are the most involved compounds in fighting the fungal infection. Germacrenes ( $\beta$-elemene and germacrene $\mathrm{D}$ ) were also not detected in control trees for most days. Another notable change was the disappearance of the monoterpene limonene following inoculation in the five testing trees. These results suggest that inoculation with $R$. lauricola causes increased energy to be used towards the MVA pathway of a tree, increasing the variety and amount of sesquiterpenes produced as a result.

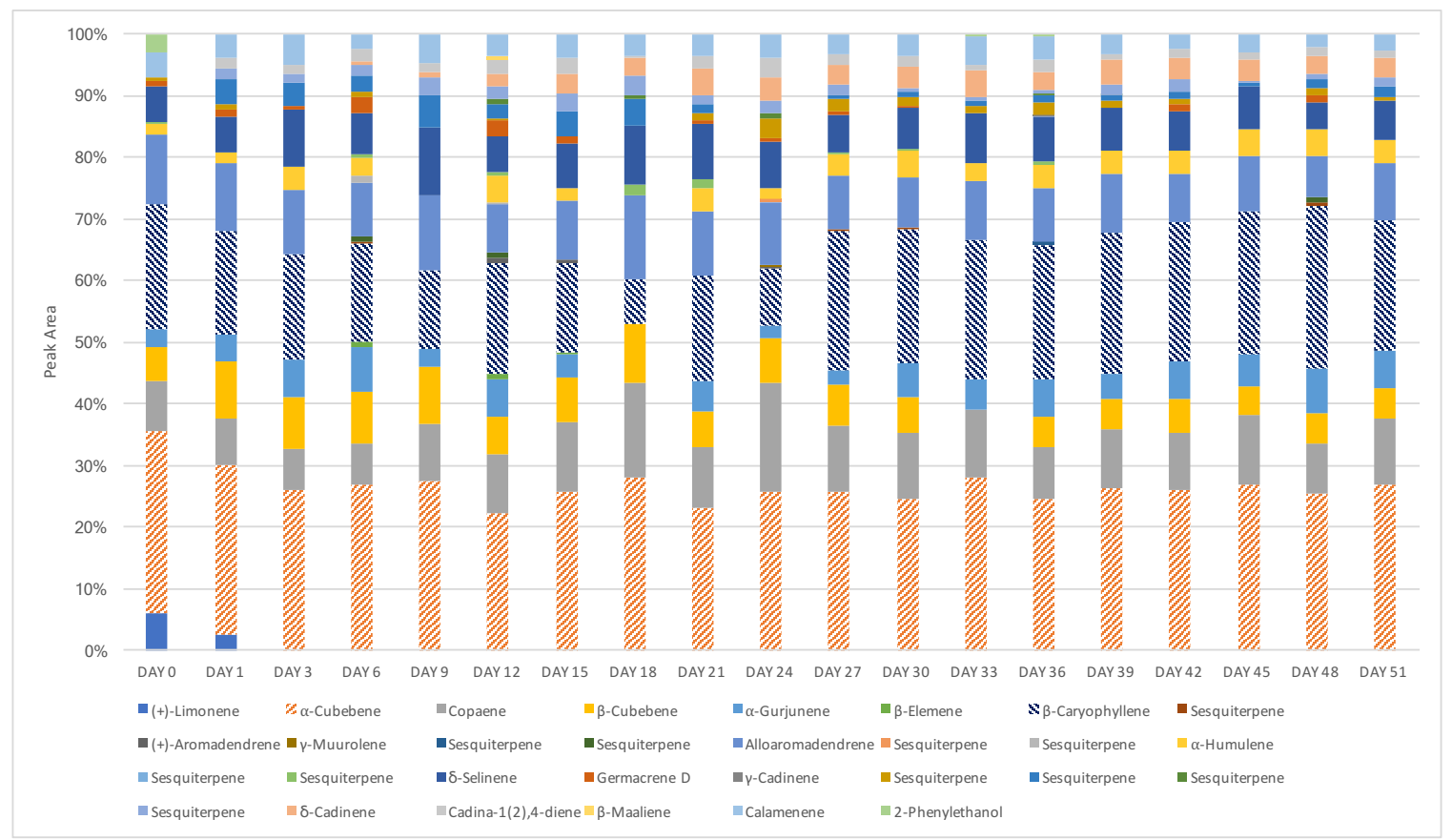

Figure 50: Odor profile of avocado trees inoculated with $\boldsymbol{R}$. lauricola by sample day. 


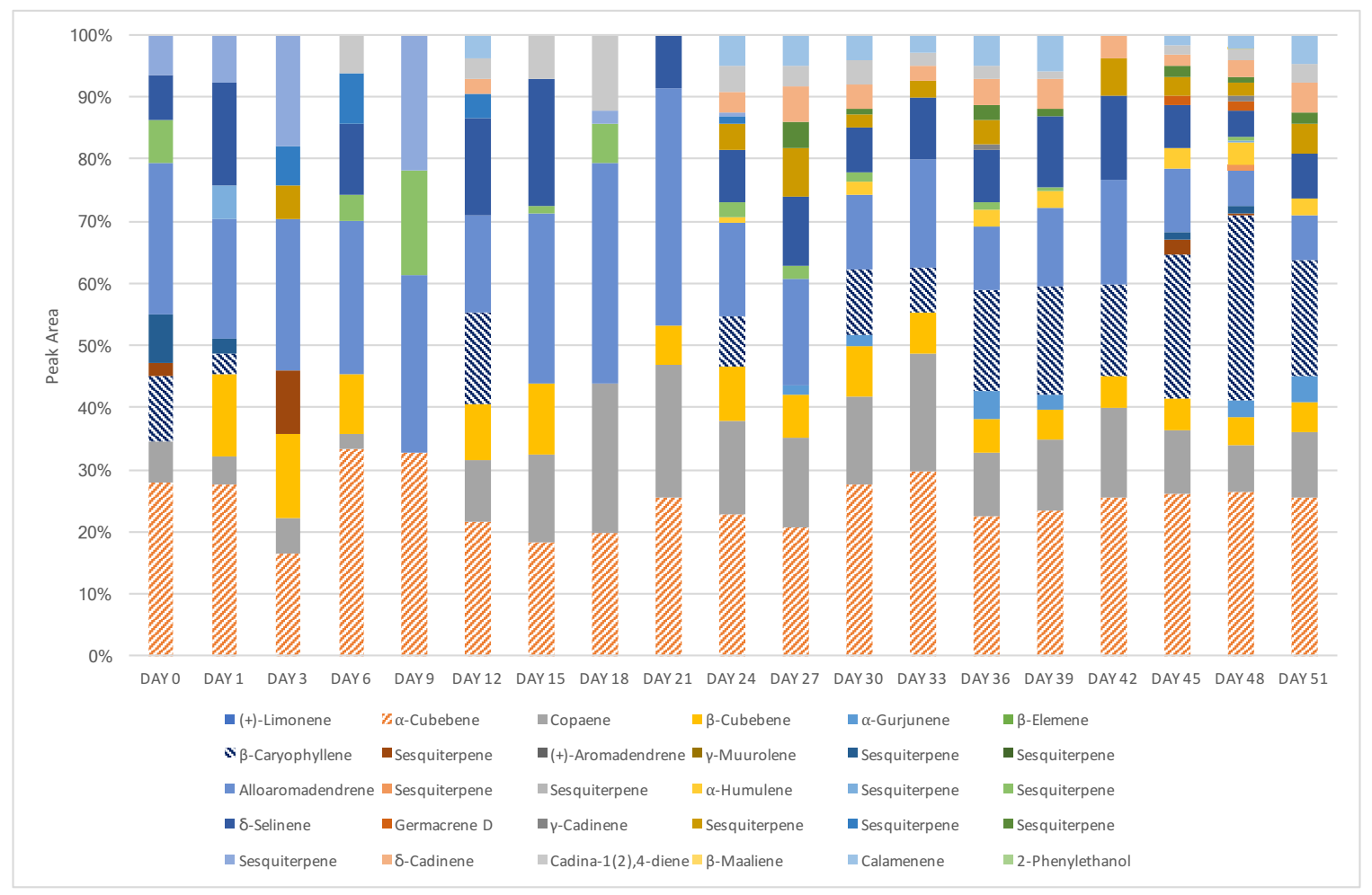

Figure 51: Odor profile of control avocado trees by sample day.

Beginning day 21, it was observed that the control trees were infested with aphids and mealy bugs. For both the infected trees and the control trees with insect infestation, VOC abundance peaked approximately two weeks after the injury was introduced (Appendix Figure 66 and Figure 67). Specifically, trees were inoculated with $R$. lauricola on Day 0, and VOC production peaked on Day 12. Insects were first observed on control trees on Day 21, and VOC production peaked on Day 36. Trees showed some immediate response in increased volatiles, then decreased VOC production the sample day before peaking. After the maximum production day, both sets of trees again decreased production for three sample days before trending upward again. The peaking of volatile production is 
a possible explanation for why the final sample days of the control trees increases so dramatically; it is a response to the insect infestation.

When analyzed using PCA (data log normalized), three separate groups could be seen (Figure 52). The inoculated and control trees days 0-21 were separated by PCA1, which explains $31.9 \%$ of the variation. PCA2 (explaining $13.8 \%$ of the variation), however, separated inoculated trees and control trees days 27-51. Complete separation was achieved with two exceptions: control trees on days 12 and 24 . The separation in control groups began on Day 27, the day when VOC production drops before the peak on Day 30 in response to insect infestation, discussed above. The observation implied that injured trees, whether they are injured by inoculation with a fungus or by insect infestation, could be separated from healthy trees through odor (driven by PCA1). Even more interesting was that inoculation and insect infested trees could be separated using PCA2. To accentuate these conclusions, a dendrogram was also created, which shows relations between samples. In Figure 53, the relationships between inoculated and control samples are clearly delineated in the same groups as the PCA in Figure 52.

Further investigation showed that the largest drivers to PCA1 and PCA2 were $\alpha-$ cubebene and $\beta$-caryophyllene (Appendix Figure 71). The influence of those two compounds was also clearly shown by the odor profiles in Figure 50 and Figure 51, where these two compounds are distinguished with hatch marks. $\beta$-caryophyllene's influence was not a surprise, since it is a humulene. As noted, humulenes were present more often in inoculated or infected trees than in control trees. However, $\beta$-caryophyllene appeared consistently in control trees after day 30, a week after insect infestation occurred, implying that humulenes are important for defense against both types of injury. However, the influence of $\alpha$ - 
cubebene was unexpected since it is a guaiene. Since the percent make-up of the odor profiles for $\alpha$-cubebene was consistent, the influence was most likely due to the amount of the compound produced rather than the observed ratio. To a lesser extent, copaene also drives the separation along PCA1. Again, the influence of copaene was expected since it is the main RAB attractant. Since copaene was produced by all avocado trees in the study, the influence was once again most likely due to the amount detected rather than the ratio.

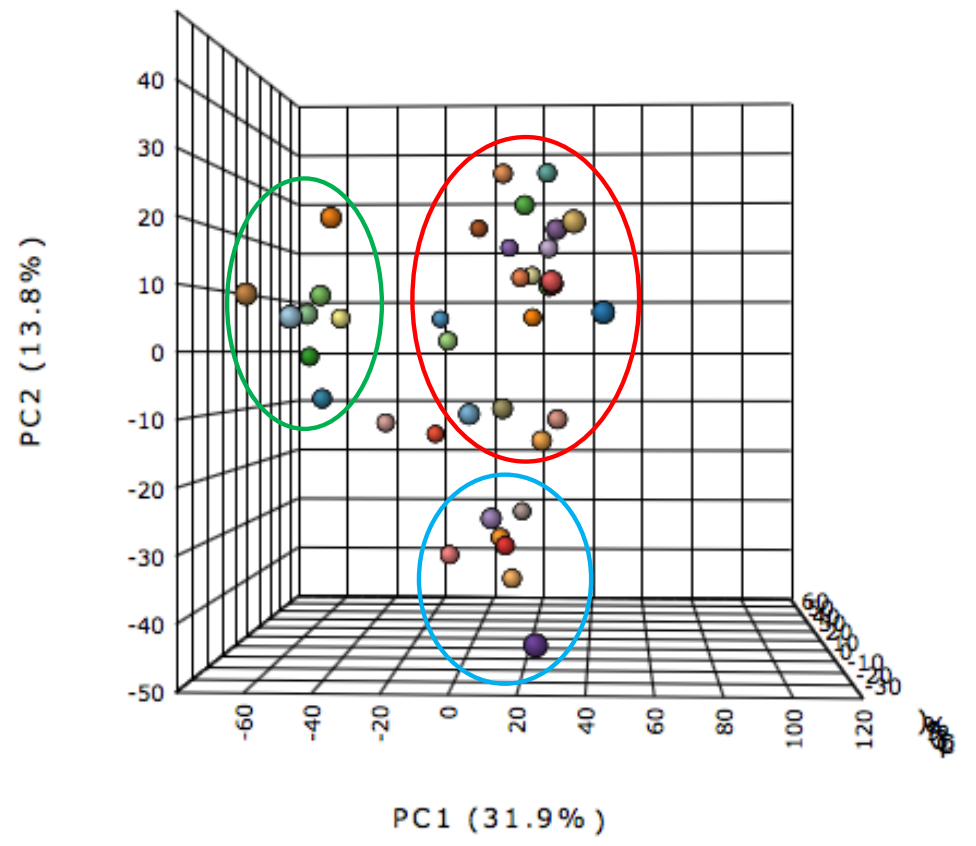

Figure 52: PCA of inoculated and control samples from the greenhouse experiment. The red circle contains inoculated samples. The green circle contains control samples from days $0-21$. The blue circle contains control samples from days 27-51. 


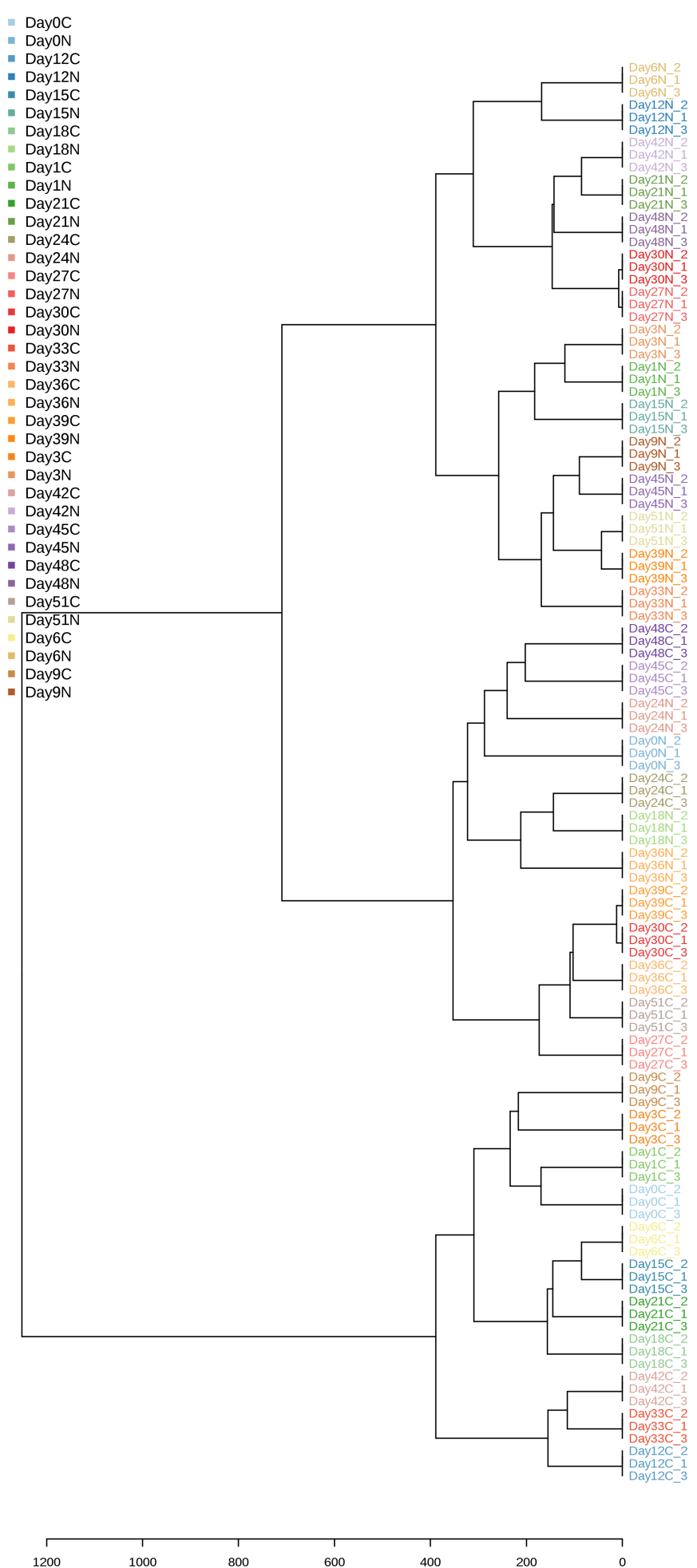

Figure 53: Dendrogram showing relations between samples of inoculated and control trees. 
As the study continued, disease progression was monitored using visual differences. Differences could be detected in the leaves of inoculated trees as laurel wilt began to take effect. Inoculated tree leaves began to display epinasty, a condition in plants where cells on the upper side of a leaf continue to grow and absorb sunlight while the lower cells cease growth to conserve energy, which can help in fighting disease or infection. Leaves with epinasty appear darker in color and begin to wilt downward. Examples of this are shown in Figure 54. To confirm the presence of the fungus in the inoculated trees, and the absence of the fungus in the control trees, SEM images were taken for each sample at the conclusion of the experiment. In each inoculated tree, tyloses were observed, while in each control tree, they were absent (examples given in Figure 55). Tyloses indicate that a tree is blocking its xylem vessels to stop the flow of an infection, such as the fungus $R$. lauricola.

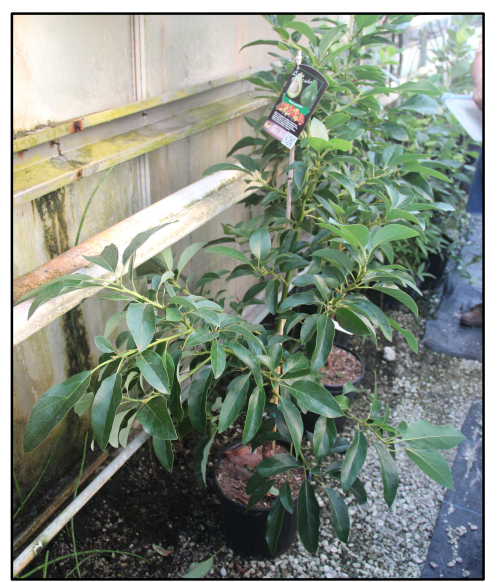

Figure 54: Images of the trees on day 0 (left); one inoculated tree after three weeks (middle); and one control tree after three weeks (right).
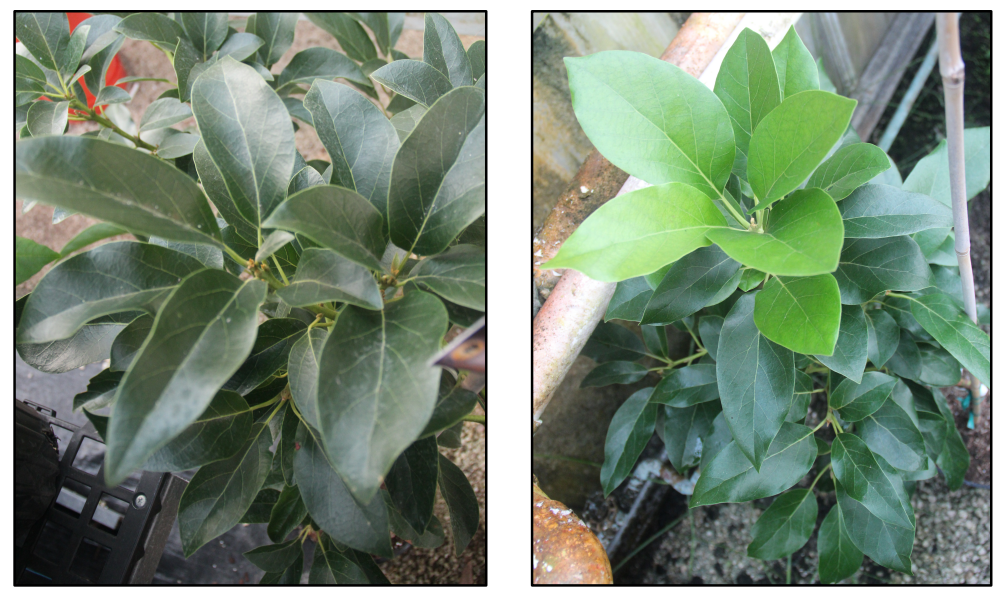

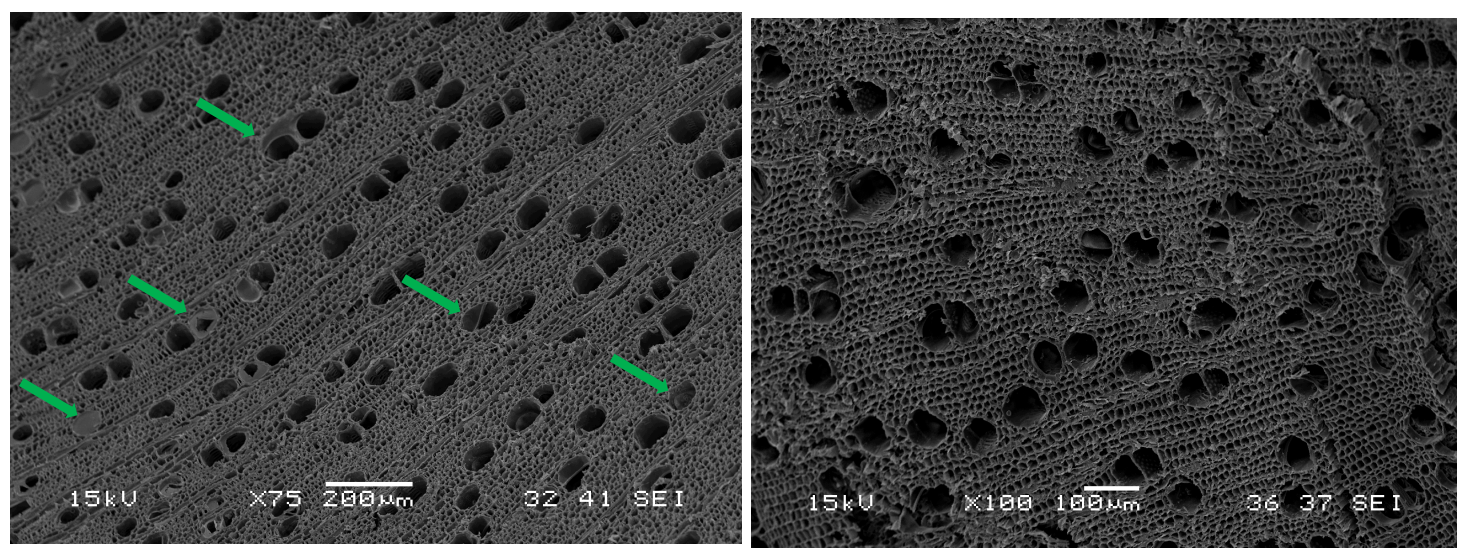

Figure 55: SEM images of inoculated tree (left) and control tree (right). Green arrows indicate some of the tyloses that can be seen in the image.

The results of the experiment showed that in young avocado trees, it was possible to use VOCs to identify trees inoculated with $R$. lauricola. These results were also consistent with the adult trees sampled in Section 5.2.2, showing that avocado trees of all ages reacted similarly to the laurel wilt disease. The reactions identified in the study can be cautiously applied to adult trees in the field. Further, VOCs could be used to separate different types of tree injuries, implying that different biochemical pathways are activated depending on the type of injury incurred. Future research should be done to prove these links and show how various chemicals are driven by interactions with the tree's environment. These outcomes should be applied to detection of the laurel wilt disease. The main compounds driving the differences in odor profiles, $\alpha$-cubebene and $\beta$-caryophyllene, could also be active odorants used by canines to identify the laurel wilt disease. Understanding the progression of volatiles during the initial period after inoculation is the first step in early identification efforts. 
5.4.2. Examining the Volatile Organic Compounds of Treated Inoculated Avocado Trees

The previous experiment showed how VOCs of young avocado trees change when inoculated with $R$. lauricola. Since there is anecdotal evidence that canines no longer alert to avocado trees treated with fungicide, the purpose of the experiment was to explore how fungicidal treatment of inoculated trees may alter VOCs. Eleven trees, four inoculated and untreated, four inoculated and treated, and three treated controls, were observed over a five week period.

Odor profiles for each group are provided in Figure 56 (trees inoculated with $R$. lauricola then treated with propiconazole), Figure 57 (trees inoculated with $R$. lauricola and left untreated), and Figure 58 (control trees not inoculated but still treated). For all inoculated trees, the odor profiles showed that trees produced fewer VOCs on Day 3, but drastically increased volatile production on Days 5 and 7 before decreasing again (Appendix Figure 68, Figure 69, and Figure 70). The pattern indicated that the trees initially reacted by slowing the biological processes leading to VOC production, but that they then increase those pathways in attempts to fight the fungus. The VOC production pattern was consistent with the results from the previous experiment. After some trees were treated on Day 7, a difference could be seen in those that were treated and those left untreated. The treated trees continued to produce more volatiles than the untreated trees, indicating that fungicidal treatment did help trees to fight the fungus, possibly by allowing them to increase efforts involved in the MVA pathway. All inoculated trees displayed a second increase in volatile production on Day 29, which was also consistent with the previous experiment. However, 
the amount of VOCs produced by the treated trees was over twice the amount produced by those left untreated, implying that treatment with propiconazole promoted the trees' ability to fight the disease through VOC production. Interestingly, the control trees drastically slowed VOC production following treatment. The pattern could indicate that propiconazole only assists the defense efforts of a tree if the tree is already involved in fighting the disease.

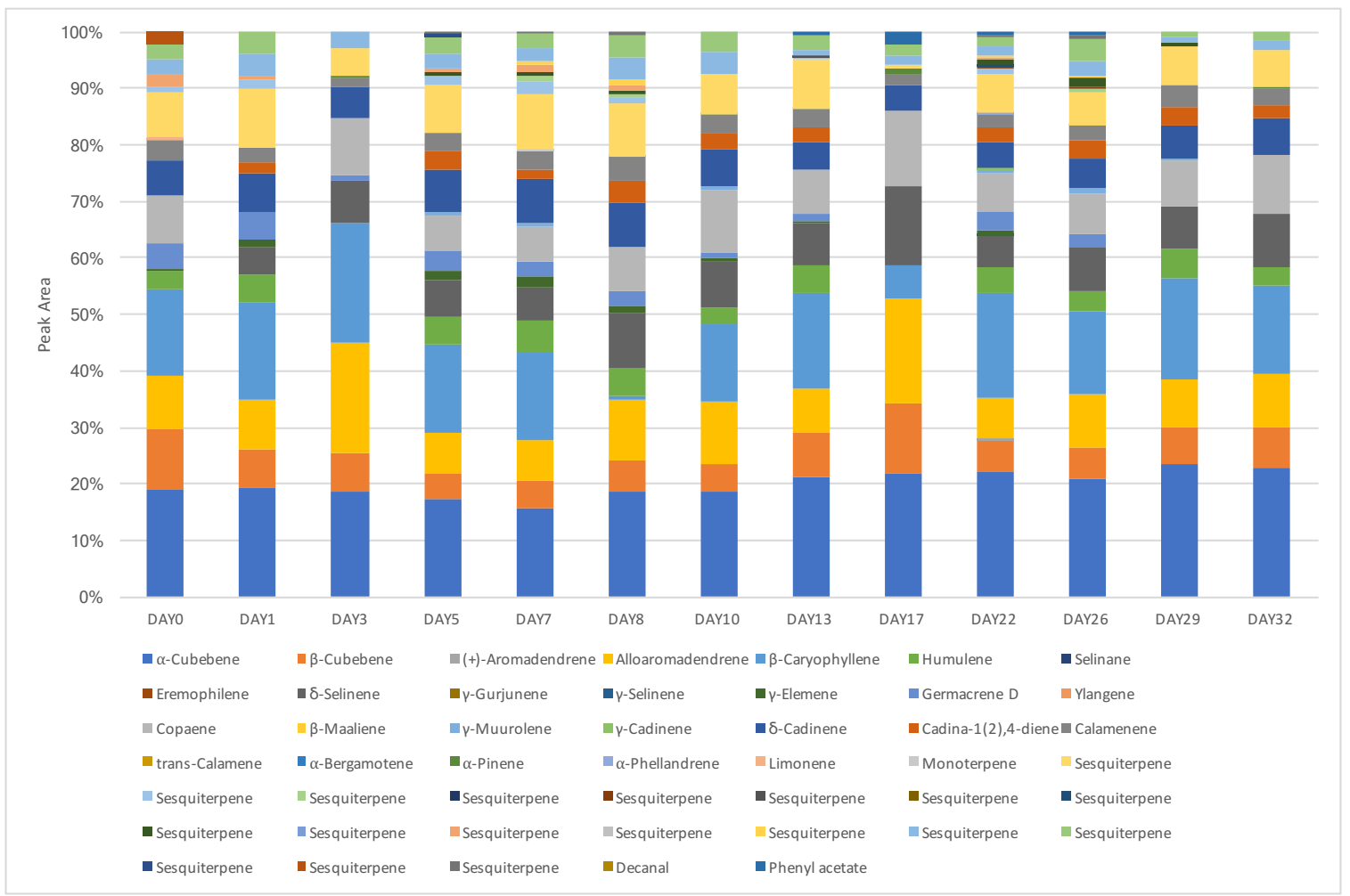

Figure 56: Odor profile of avocado trees inoculated with $\boldsymbol{R}$. lauricola and then treated with propiconazole by sample day. 


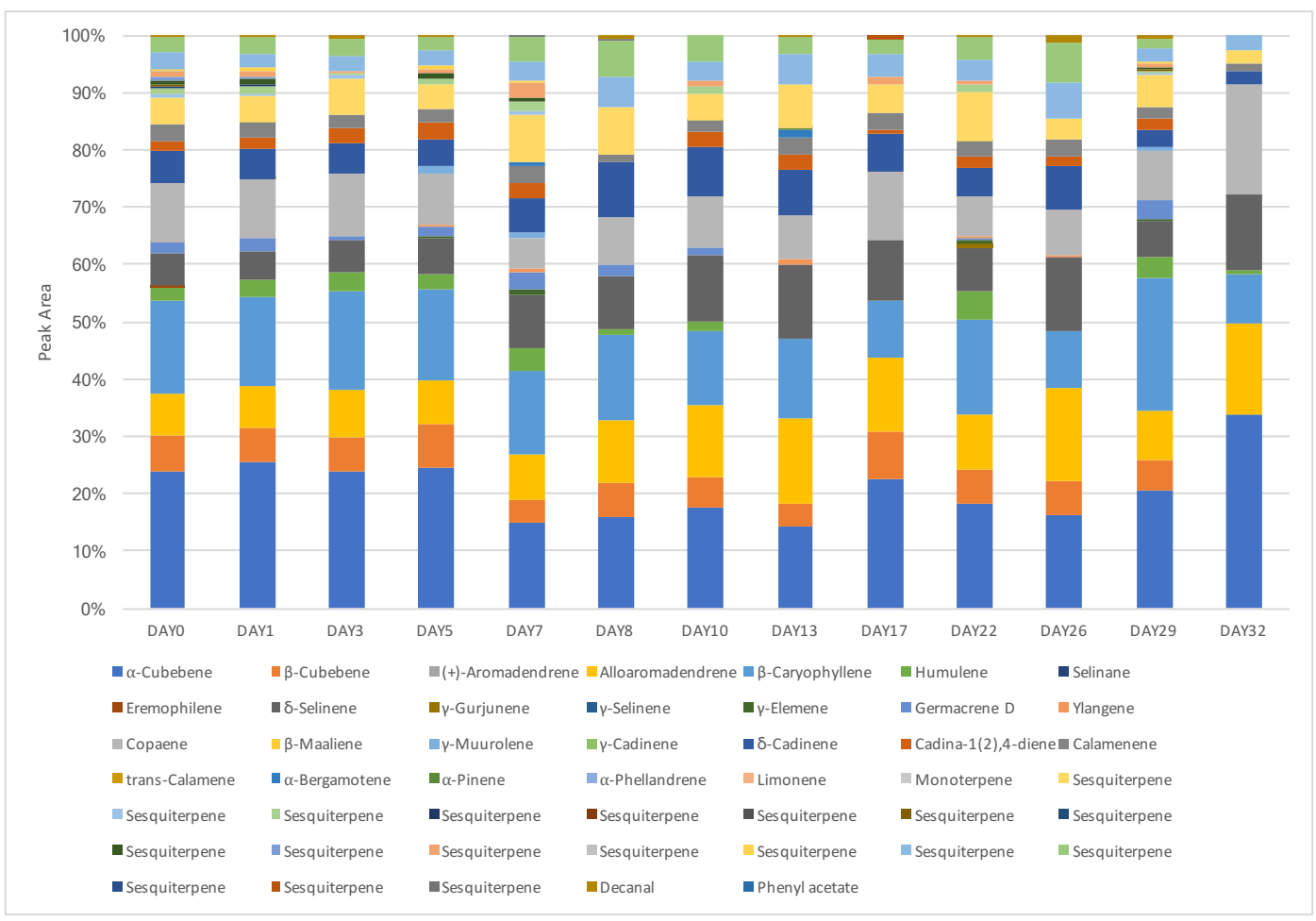

Figure 57: Odor profile of avocado trees inoculated with $\boldsymbol{R}$. lauricola and left untreated by sample day.

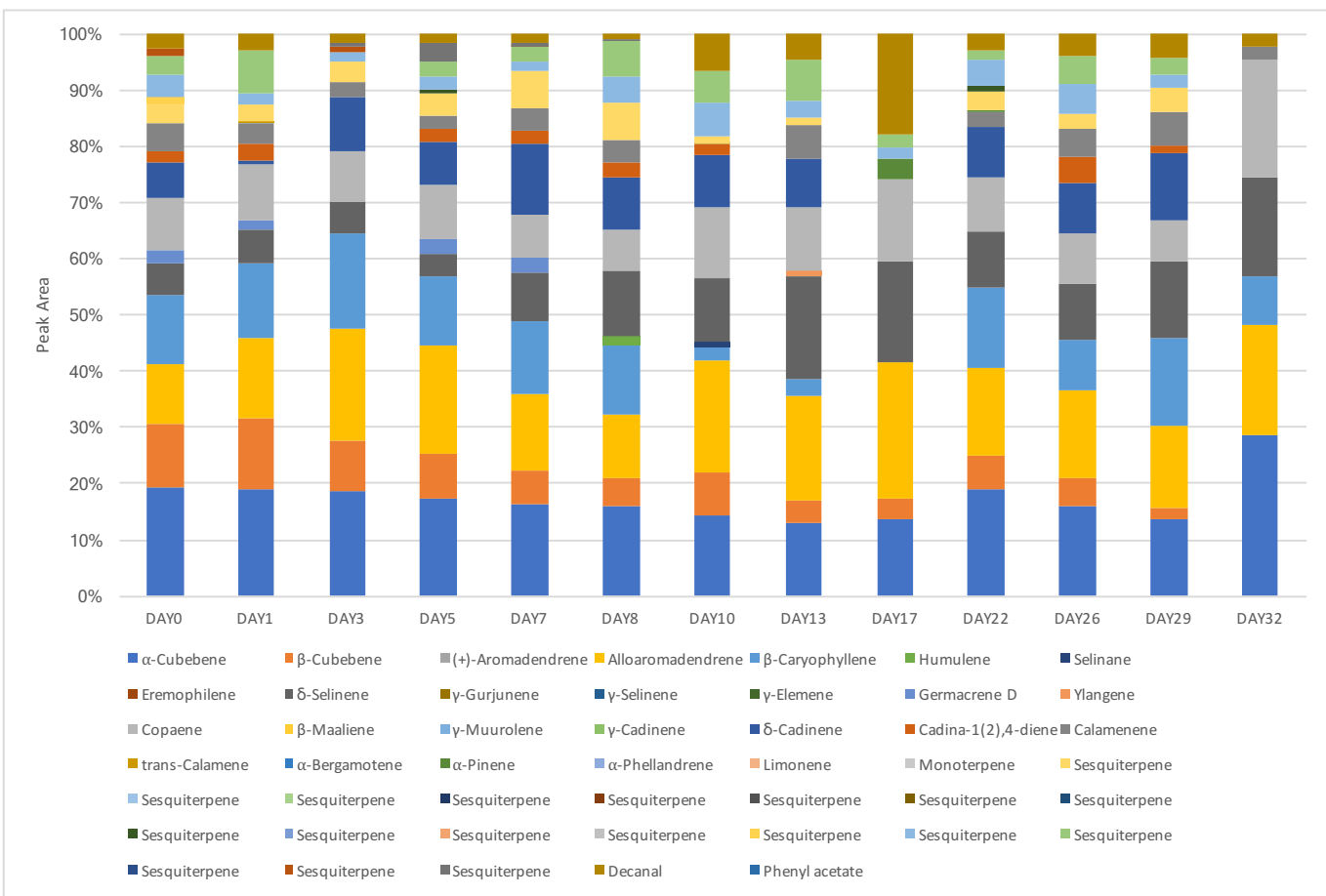

Figure 58: Odor profile of control avocado trees (not inoculated with $R$. lauricola but treated with propiconazole) by sample day. 
A PCA plot of the data (log normalized) shows that less separation was achieved between the sample set than the inoculated and control trees in the previous experiment (Figure 59). While the control samples grouped tightly, the inoculated samples (both treated and untreated) grouped less clearly. However, there was some separation based on PCA1 (32\% of variation) and PCA2 (10.3\% of variation). Controls were separated from the inoculated trees based on PCA1. Inoculated trees were separated into treated and untreated samples by PCA2, though the association was not strong. The separation is interesting since it indicated that the VOCs of all inoculated trees were similar, whether or not they had been treated with propiconazole. Since PCA1 did not explain all of the separation between the inoculated and treated samples and control but treated samples, it was also determined that treatment did affect the volatile production of any tree, whether or not it was inoculated. The results support the idea that canines do not alert to trees that have been treated with fungicide, since their VOC production has been affected. Consistent with the results from the previous greenhouse experiment, $\beta$-caryophyllene and $\alpha$-cubebene were the biggest drivers of separation along PCA1 (Appendix Figure 72). Other influences included an unidentified sesquiterpene (RT18.233), $\delta$-selinene, $\delta$-cadinene, and alloaromadendrene. The variety of sesquiterpene categories that these compounds come from suggests that fungicidal treatment increased MVA activity, but the activation was unfocused and did not target any one method of volatile defense. 


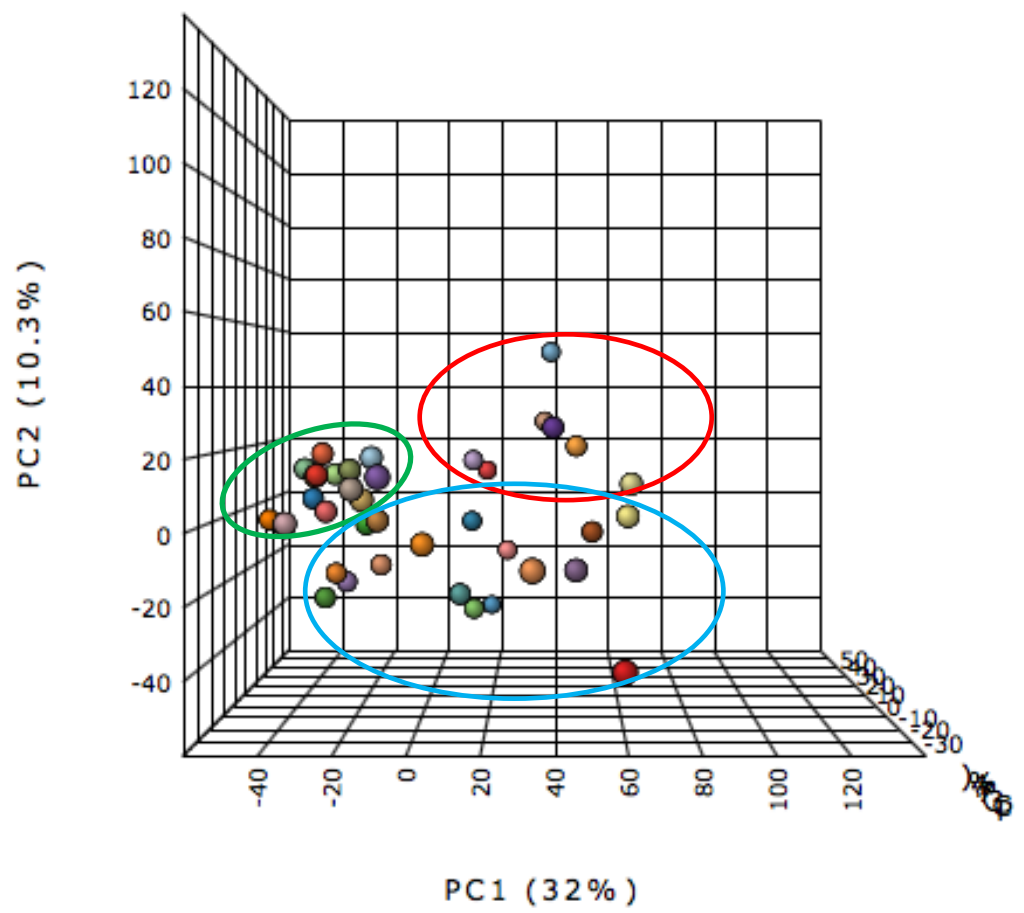

Figure 59: PCA of inoculated then treated, inoculated then untreated, and control/treated samples from the greenhouse experiment. The red circle contains inoculated but untreated samples. The green circle contains control/treated samples. The blue circle contains inoculated and treated samples.

At the conclusion of the study, SEM images were taken of each tree to determine the presence or absence of tyloses, which indicate the presence or absence of $R$. lauricola in the trees (examples given in Figure 60). Tyloses were clearly present in all inoculated trees, whether or not they were treated with the fungicide, while they were absent in the control tree, even though it was treated. Additionally, there were more tyloses present in the untreated trees, indicating that the treatment did indeed slow the progression of disease. Images of the trees taken throughout the study show symptoms of laurel wilt such as epinasty on the inoculated, untreated trees (Figure 61). Both sets of treated trees show darker leaves, but there is no curling or wilting as is present in the inoculated trees. 

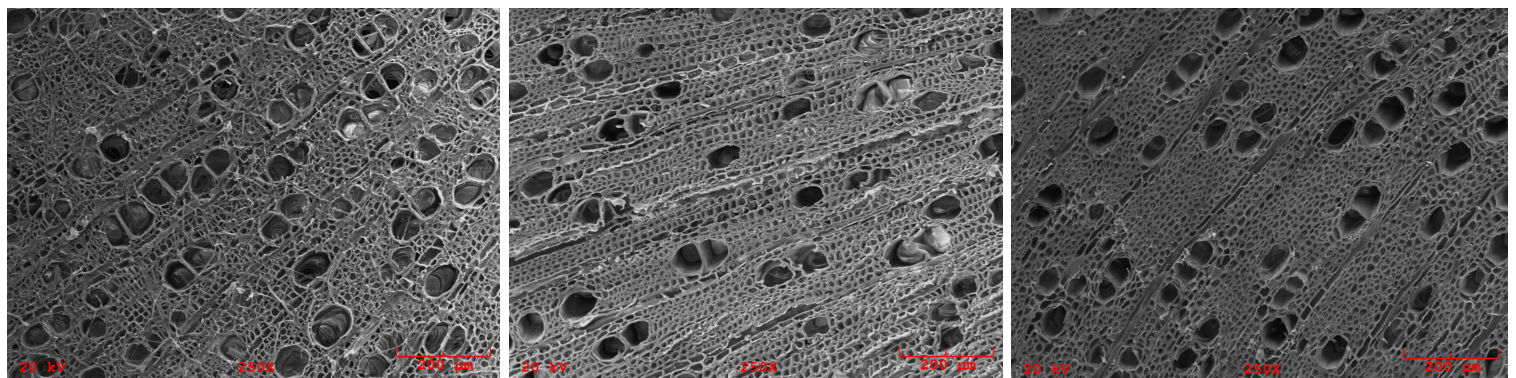

Figure 60: SEM images of inoculated and untreated tree (left), inoculated and treated tree (middle), and control but treated tree (right).
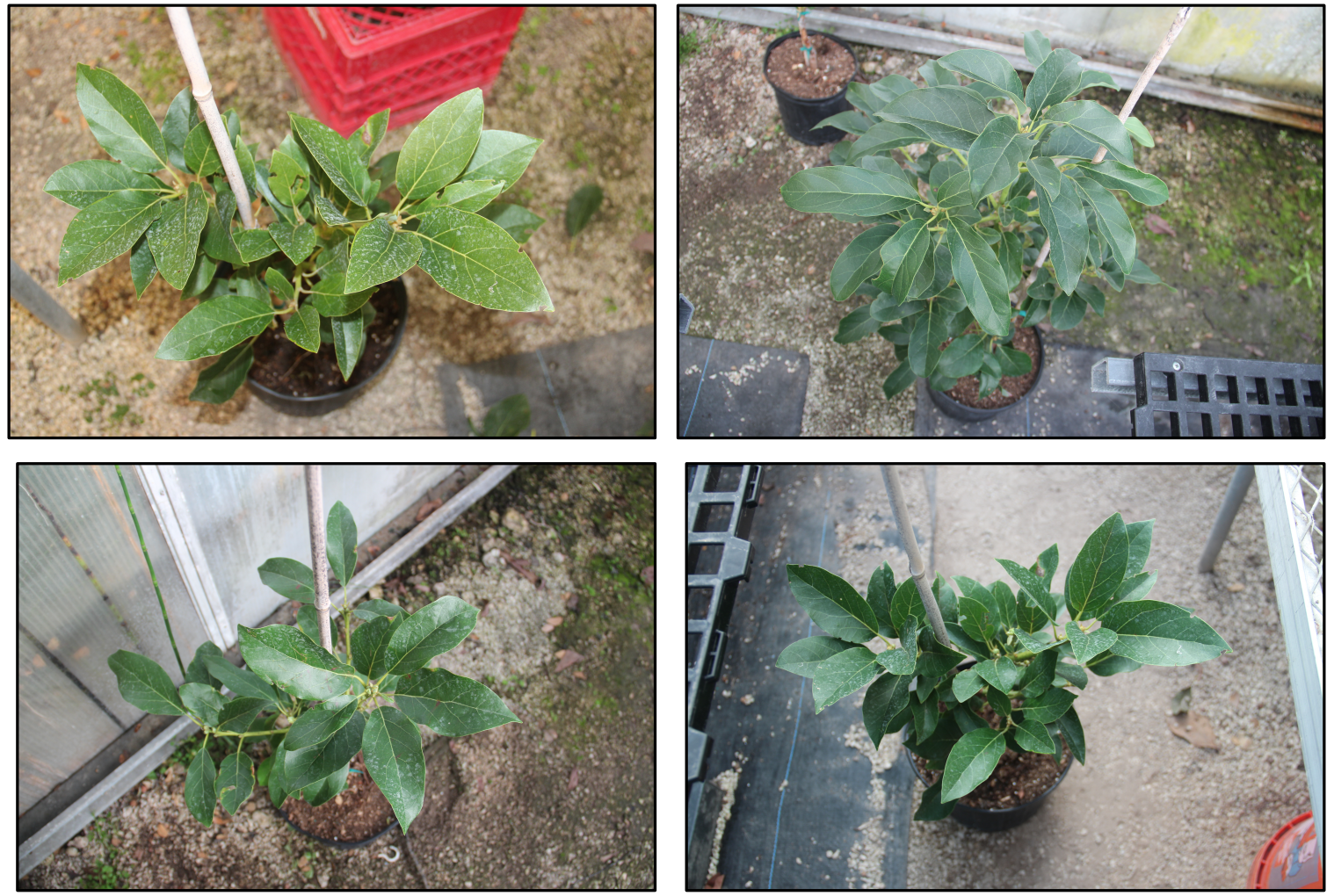

Figure 61: Images of the trees (clockwise from upper left) on day 0; one inoculated tree on day 32; one inoculated then treated tree on day 32; and one control tree on day 32.

The results of the study were consistent with the results from the previous greenhouse experiment. There were clear trends in volatile production resulting from inoculation with R. lauricola and treatment with propiconazole. Identified VOCs are in line 
with those detected in Section 5.4.1, as are the volatiles that drive the differences and separation in odor profiles ( $\beta$-caryophyllene and $\alpha$-cubebene). The SEM images showed that the inoculated trees were in fact infected with laurel wilt disease. These results can be used to support the notion that treatment with a fungicide alters the VOC profile of trees, meaning that canines will not alert to those trees. The information contributed to the understanding of how $R$. lauricola and young avocado trees interact with each other, which could influence how detection and treatment of the disease is approached in the field.

\section{CONCLUSION}

While the use of detection canines for environmental and agricultural applications is limited, the dissertation aimed to contribute to the understanding of VOCs in such settings in order to widen the scope of canine detection utilization and pest detection. Through the results and experiments presented in Section 5, VOC profiles were created for $R$. lauricola, young and adult infected avocado trees, young and adult uninfected trees, and young inoculated trees treated with propiconazole. From the odor profiles, trends were established and influential volatile compounds were identified, both of which can be applied to help curb the spread of the laurel wilt disease.

Seventeen compounds were identified in the headspace above $R$. lauricola over an initial 28 day growth period, showing that in this time, VOCs alter dramatically, changing from alcohols, acetates, and esters to terpenes. Terpenes are traditionally formed as secondary metabolites from the MVA pathway, possibly revealing a switch from active development to morphological development of the fungus. PCA and cloud plots visually 
demonstrated these changes, clustering headspace samples by weekly growth patterns in VOCs and showing toggle up- and down-regulated compounds. These results proved that the headspace of $R$. lauricola is more complex than originally reported in the literature ${ }^{26}$, and formed a basis of understanding for how avocado trees may respond to early stages of inoculation with the pathogen.

Infected and uninfected avocado trees were next analyzed for headspace volatiles, identifying six compounds present in infected Lula variety trees, and eight present in uninfected samples of the same variety. All of these compounds were sesquiterpenes, and their category of formation was identified and described. The only compound unique to infected trees was caryophyllene oxide from the humulene category. Compounds unique to uninfected samples were $\alpha$-selinene, $\alpha$-cubebene, and (-)-alloaromadendrene, from the eudesmene and guaiene categories. These categories of formation were exclusive to their respective samples, revealing which pathways could be more or less important in defense against the laurel wilt disease. Based on these results, humulenes appear essential to fighting $R$. lauricola, while eudesmenes and guaienes are not. The present research was the first study to identify VOCs in the headspace of avocado trees infected with $R$. lauricola.

Further studies expanded the knowledge base through greenhouse studies of inoculated avocado trees and inoculated trees treated with propiconazole. Consistent with findings from adult trees with laurel wilt, these samples displayed higher humulene production in inoculated trees, and lower guaiene production. Separation of inoculated trees, control trees, and control trees with insect infestation was done using PCA, driven by $\beta$-caryophyllene and $\alpha$-cubebene. Further separation was done of inoculated trees, inoculated and treated trees, and control trees. Additionally, the amount of volatiles 
produced displays a clear pattern of decreases and increases following an injury. The information proves trees inoculated with $R$. lauricola respond using VOCs. The information gained through these studies adds to the existing literature a greater understanding of how avocado trees react to inoculation with $R$. lauricola, which can be used to help bolster tree defenses and agricultural defense systems.

Once a basic understanding of the volatiles produced by the various systems (i.e. $R$. lauricola and avocado trees) was determined, the focus shifted to standoff detection of the laurel wilt disease. COMPS were evaluated to determine shelf-life and dissipation rates of the target odors, and a new method of creating safer canine training aids was established. Static sampling of COMPS revealed that training aids had a permeation rate of $3014 \mathrm{~A} / \mathrm{E}$ per day. Based on the results, recommendations were made to create the aids a day in advance, and discard them after four days of use. Using the dynamic method of sampling (with storage in non-permeable packaging between samples), the dissipation rate was $15703 \mathrm{~A} / \mathrm{E}$ per day. The method extended the shelf-life a day, despite the higher permeation rate. The characterization of the target will help to inhibit the spread of laurel wilt disease through improved COMPS efficiency, which will in turn improve use of canines as the main source of early disease identification and standoff detection, helping to protect economic and agricultural interests.

The development of the column vent method for rapid odorant identification was an essential process that can significantly change how canine training in agricultural and environmental detection is approached. Fractions of the odor were collected on cotton gauze in glass vials from a vented chromatographic column. Then, the fractions were presented in trials for canine detection. The process was designed to allow canines to select 
chromatographic areas of interest from samples of infected avocado trees, without having to obtain all compounds of interest commercially. It also makes the time-consuming process of odorant identification faster. The fractions the canines alerted to, A ( $50 \%$ alert rate) and $\mathrm{B}$ (33.3\% alert rate), can now be used in mimic training aids, reducing dependence on live species or sources as training aids. The results will allow greater application of environmental and agricultural detection canines by addressing common issues, such as the rarity of the species, legality of obtaining and maintaining the species, methods of acquisition, and the possibility of spreading the species should it escape the containment system. These methods are also well suited for other novel pests, diseases, and pathogens where standoff or rapid detection is necessary but challenging.

The dissertation presented information necessary to the detection and understanding of the laurel wilt disease in commercial avocado groves. Standoff detection of infected trees using volatiles is essential in large groves where the presence or location of disease is unknown, and time is limited. It is important to constrain the spread of $R$. lauricola in groves, which can only be done through early detection methods. By first building a base of knowledge consisting of the volatile reactions of the trees to inoculation and then applying that to canine detection, rapid, early identification is possible. Knowledge of the disease and standoff volatile detection capabilities are enhanced using the information, and crop defenses are improved. 


\section{REFERENCES}

1. Hulcr, J.; Dunn, R. R. The sudden emergence of pathogenicity in insect-fungus symbioses threatens naive forest ecosystems. Proceedings of the Royal Society of London B: Biological Sciences 2011, 278 (1720), 2866-2873.

2. Cheng, A.-X.; Lou, Y.-G.; Mao, Y.-B.; Lu, S.; Wang, L.-J.; Chen, X.-Y. Plant terpenoids: biosynthesis and ecological functions. Journal of Integrative Plant Biology 2007, 49 (2), 179-186.

3. Paschold, A.; Halitschke, R.; Baldwin, I. T. Using 'mute'plants to translate volatile signals. The Plant Journal 2006, 45 (2), 275-291.

4. Davis, T. S.; Crippen, T. L.; Hofstetter, R. W.; Tomberlin, J. K. Microbial volatile emissions as insect semiochemicals. Journal of chemical ecology 2013, 39 (7), 840-859.

5. Korpi, A.; Järnberg, J.; Pasanen, A. L. Microbial volatile organic compounds. Critical reviews in toxicology 2009, 39 (2), 139-193.

6. Keller, N. P.; Turner, G.; Bennett, J. W. Fungal secondary metabolism--from biochemistry to genomics. Nature Reviews Microbiology 2005, 3 (12), 937-947.

7. Köllner, T. G.; Schnee, C.; Gershenzon, J.; Degenhardt, J. The variability of sesquiterpenes emitted from two Zea mays cultivars is controlled by allelic variation of two terpene synthase genes encoding stereoselective multiple product enzymes. The Plant Cell 2004, 16 (5), 1115-1131.

8. Demyttenaere, J. C.; Adams, A.; Van Belleghem, K.; De Kimpe, N.; König, W. A.; Tkachev, A. V. De novo production of (+)-aristolochene by sporulated surface cultures of Penicillium roqueforti. Phytochemistry 2002, 59 (6), 597-602.

9. Sunesson, A.; Vaes, W.; Nilsson, C.; Blomquist, G.; Andersson, B.; Carlson, R. Identification of volatile metabolites from five fungal species cultivated on two media. Applied and Environmental Microbiology 1995, 61 (8), 2911-2918.

10. Müller, A.; Faubert, P.; Hagen, M.; zu Castell, W.; Polle, A.; Schnitzler, J.-P.; Rosenkranz, M. Volatile profiles of fungi-chemotyping of species and ecological functions. Fungal Genetics and Biology 2013, 54, 25-33.

11. Dudareva, N.; Pichersky, E.; Gershenzon, J. Biochemistry of plant volatiles. Plant physiology 2004, 135 (4), 1893-1902.

12. Morath, S. U.; Hung, R.; Bennett, J. W. Fungal volatile organic compounds: a review with emphasis on their biotechnological potential. Fungal Biology Reviews 2012, 26 (2), 73-83. 
13. Baldwin, I. T.; Halitschke, R.; Paschold, A.; Von Dahl, C. C.; Preston, C. A. Volatile signaling in plant-plant interactions:" talking trees" in the genomics era. Science 2006, 311 (5762), 812-815.

14. Schnürer, J.; Olsson, J.; Börjesson, T. Fungal volatiles as indicators of food and feeds spoilage. Fungal Genetics and Biology 1999, 27 (2-3), 209-217.

15. Heil, M.; Kost, C. Priming of indirect defences. Ecology letters 2006, 9 (7), 813817.

16. Kost, C.; Heil, M. Herbivore-induced plant volatiles induce an indirect defence in neighbouring plants. Journal of Ecology 2006, 94 (3), 619-628.

17. Hung, R.; Lee, S.; Bennett, J. W. Fungal volatile organic compounds and their role in ecosystems. Applied microbiology and biotechnology 2015, 99 (8), 33953405.

18. Niogret, J.; Kendra, P. E.; Epsky, N. D.; Heath, R. R. Comparative analysis of terpenoid emissions from Florida host trees of the redbay ambrosia beetle, Xyleborus glabratus (Coleoptera: Curculionidae: Scolytinae). Florida Entomologist 2011, 94 (4), 1010-1017.

19. Niogret, J.; Epsky, N. D.; Schnell, E. Q.; Schnell, R. J.; Heath, R. R.; Meerow, A. W.; Kendra, P. E. Analysis of Sesquiterpene Distributions in the Leaves, Branches, and Trunks of Avocado (Persea americana Mill.). 2013.

20. Niogret, J.; Epsky, N. D.; Schnell, R. J.; Boza, E. J.; Kendra, P. E.; Heath, R. R. Terpenoid variations within and among half-sibling avocado trees, Persea americana Mill.(Lauraceae). PloS one 2013, 8 (9), e73601.

21. Polizzi, V.; Adams, A.; De Saeger, S.; Van Peteghem, C.; Moretti, A.; De Kimpe, $\mathrm{N}$. Influence of various growth parameters on fungal growth and volatile metabolite production by indoor molds. Science of the Total Environment 2012, 414, 277-286.

22. Calvo, A. M.; Wilson, R. A.; Bok, J. W.; Keller, N. P. Relationship between secondary metabolism and fungal development. Microbiology and molecular biology reviews 2002, 66 (3), 447-459.

23. Six, D. L. Ecological and evolutionary determinants of bark beetle-fungus symbioses. Insects 2012, 3 (1), 339-366.

24. Simon, A. G.; Mills, D. K.; Furton, K. G. Chemical and canine analysis as complimentary techniques for the identification of active odors of the invasive fungus, Raffaelea lauricola. Talanta 2017. 
25. Simon, A. G.; Mills, D. K.; Furton, K. G. Chemotyping the temporal volatile organic compounds of an invasive fungus to the United States, Raffaelea lauricola. Journal of Chromatography A 2017, 1487, 72-76.

26. Kuhns, E. H.; Tribuiani, Y.; Martini, X.; Meyer, W. L.; Peña, J.; Hulcr, J.; Stelinski, L. L. Volatiles from the symbiotic fungus Raffaelea lauricola are synergistic with manuka lures for increased capture of the redbay ambrosia beetle Xyleborus glabratus. Agricultural and Forest Entomology 2014, 16 (1), 87-94.

27. Pimentel, D.; Zuniga, R.; Morrison, D. Update on the environmental and economic costs associated with alien-invasive species in the United States. Ecological economics 2005, 52 (3), 273-288.

28. Hulcr, J.; Dunn, R. R. The sudden emergence of pathogenicity in insect-fungus symbioses threatens naive forest ecosystems. Proceedings of the Royal Society of London B: Biological Sciences 2011, 278 (1720), 2866-2873.

29. Ploetz, R. C.; Hulcr, J.; Wingfield, M. J.; de Beer, Z. W. Destructive tree diseases associated with ambrosia and bark beetles: black swan events in tree pathology? Plant Disease 2013, 97 (7), 856-872.

30. Hughes, M. A.; Riggins, J. J.; Koch, F. H.; Cognato, A. I.; Anderson, C.; Formby, J. P.; Dreaden, T. J.; Ploetz, R. C.; Smith, J. A. No rest for the laurels: symbiotic invaders cause unprecedented damage to southern USA forests. Biological Invasions 2017, 1-15.

31. Wuest, C. E.; Harrington, T. C.; Fraedrich, S. W.; Yun, H. Y.; Lu, S. S. Genetic Variation in Native Populations of the Laurel Wilt Pathogen, Raffaelea lauricola, in Taiwan and Japan and the Introduced Population in the United States. Plant Disease 2017, 101 (4), 619-628.

32. Hughes, M. A.; Smith, J. A.; Ploetz, R. C.; Kendra, P. E.; Hanula, J.; Hulcr, J.; Stelinski, L. L.; Cameron, S.; Riggins, J. J.; Carrillo, D. Recovery plan for laurel wilt on redbay and other forest species caused by Raffaelea lauricola and disseminated by Xyleborus glabratus. 2015.

33. Evans, E. A.; Crane, J.; Hodges, A.; Osborne, J. L. Potential economic impact of laurel wilt disease on the Florida avocado industry. HortTechnology 2010, 20 (1), 234-238.

34. Mayfield Iii, A. E.; Peña, J. E.; Crane, J. H.; Smith, J. A.; Branch, C. L.; Ottoson, E. D.; Hughes, M. Ability of the redbay ambrosia beetle (Coleoptera: Curculionidae: Scolytinae) to bore into young avocado (Lauraceae) plants and transmit the laurel wilt pathogen (Raffaelea sp). Florida Entomologist 2008, 91 (3), 485-487. 
35. O'Brien, D. Addressing a new threat to avocado: laurel wilt. Agricultural Research 2012, 60 (9), 8.

36. Ploetz, R. C.; Pérez-Martínez, J. M.; Smith, J. A.; Hughes, M.; Dreaden, T. J.; Inch, S. A.; Fu, Y. Responses of avocado to laurel wilt, caused by Raffaelea lauricola. Plant Pathology 2012, 61 (4), 801-808.

37. Kendra, P.; Montgomery, W.; Schnell, E.; Niogret, J.; Epsky, N.; Heath, R.; Schnell Ii, R.; Ayala Silva, T.; Meerow, A.; Winterstein, M. USDA-ARS strategies to address the imminent threat of redbay ambrosia beetle and laurel wilt disease to avocados in Florida. 2009.

38. Kasson, M. T.; O'Donnell, K.; Rooney, A. P.; Sink, S.; Ploetz, R. C.; Ploetz, J. N.; Konkol, J. L.; Carrillo, D.; Freeman, S.; Mendel, Z. An inordinate fondness for Fusarium: phylogenetic diversity of fusaria cultivated by ambrosia beetles in the genus Euwallacea on avocado and other plant hosts. Fungal Genetics and Biology 2013, 56, 147-157.

39. Carrillo, D.; Duncan, R. E.; Ploetz, J. N.; Campbell, A. F.; Ploetz, R. C.; Peña, J. E. Lateral transfer of a phytopathogenic symbiont among native and exotic ambrosia beetles. Plant Pathology 2014, 63 (1), 54-62.

40. Kendra, P.; Narvaez, T.; Montgomery, W.; Carrillo, D. Ambrosia beetle communities in forest and agriculture ecosystems with laurel wilt disease. 2015; pp 15-18.

41. Ploetz, R. C.; Konkol, J. L.; Narvaez, T.; Duncan, R. E.; Saucedo, R. J.; Campbell, A.; Mantilla, J.; Carrillo, D.; Kendra, P. E. Presence and Prevalence of Raffaelea lauricola, Cause of Laurel Wilt, in Different Species of Ambrosia Beetle in Florida, USA. Journal of economic entomology 2017, tow 292.

42. Hulcr, J.; Mann, R.; Stelinski, L. L. The scent of a partner: ambrosia beetles are attracted to volatiles from their fungal symbionts. Journal of chemical ecology 2011, 37 (12), 1374-1377.

43. Yadeta, K. A.; Thomma, B. P. The xylem as battleground for plant hosts and vascular wilt pathogens. 2013.

44. Cuevas, Jr. R. A. Memorandum, Miami-Dade County, Agenda Item No. 11(A)(8); Oct 6,15 .

45. Price-Williams, A. Memorandum, Miami-Dade County, Agenda Item No. 7(A); Jul 19, 16.

46. Ploetz, R. C.; Konkol, J. L.; Pérez-Martínez, J. M.; Fernandez, R. Management of laurel wilt of avocado, caused by Raffaelea lauricola. European Journal of Plant Pathology 2017, 1-11. 
47. Ploetz, R. C.; Kendra, P. E.; Choudhury, R. A.; Rollins, J. A.; Campbell, A.; Garrett, K.; Hughes, M.; Dreaden, T. Laurel wilt in natural and agricultural ecosystems: understanding the drivers and scales of complex pathosystems. Forests 2017, 8 (2), 48.

48. Ploetz, R. C.; Hughes, M. A.; Kendra, P. E.; Fraedrich, S. W.; Carrillo, D.; Stelinski, L. L.; Hulcr, J.; Mayfield Iii, A. E.; Dreaden, T. L.; Crane, J. H. Recovery Plan for Laurel Wilt of Avocado, caused by Raffaelea lauricola. Plant Health Progr 2017, 18.

49. Cameron, R. S.; Bates, C.; Johnson, J. Distribution and spread of laurel wilt disease in Georgia: 2006-08 survey and field observations. Georgia Forestry Commission report 2008, 2006-2008.

50. Fraedrich, S. W. California laurel is susceptible to laurel wilt caused by Raffaelea lauricola. Plant Disease 2008, 92 (10), 1469.

51. Fraedrich, S. W.; Harrington, T. C.; Rabaglia, R. J.; Ulyshen, M. D.; Mayfield Iii, A. E.; Hanula, J. L.; Eickwort, J. M.; Miller, D. R. A fungal symbiont of the redbay ambrosia beetle causes a lethal wilt in redbay and other Lauraceae in the southeastern United States. Plant Disease 2008, 92 (2), 215-224.

52. Fraedrich, S. W.; Harrington, T. C.; Bates, C. A.; Johnson, J.; Reid, L. S.; Best, G. S.; Leininger, T. D.; Hawkins, T. S. Susceptibility to laurel wilt and disease incidence in two rare plant species, pondberry and pondspice. Plant Disease 2011, 95 (9), 1056-1062.

53. Fraedrich, S. W.; Harrington, T. C.; Best, G. S. Xyleborus glabratus attacks and systemic colonization by Raffaelea lauricola associated with dieback of Cinnamomum camphora in the southeastern United States. Forest Pathology 2015, 45 (1), 60-70.

54. Hughes, M. A.; Brar, G.; Ploetz, R. C.; Smith, J. A. Field and growth chamber inoculations demonstrate Persea indica as a newly recognized host for the laurel wilt pathogen, Raffaelea laurciola. Plant Health Progress. $w w w$. plantmanagementnetwork. org/php/elements/sum2. aspx 2013.

55. Mayfield Iii, A. E.; Smith, J. A.; Hughes, M.; Dreaden, T. J. First report of laurel wilt disease caused by a Raffaelea sp. on avocado in Florida. Plant Disease 2008, $92(6), 976$.

56. Ploetz, R. C.; Konkol, J. First report of gulf licaria, Licaria trianda, as a suscept of laurel wilt. Biological Invasions 2017, 79. 
57. Smith, J. A.; Mount, L.; Mayfield Iii, A. E.; Bates, C. A.; Lamborn, W. A.; Fraedrich, S. W. First report of laurel wilt disease caused by Raffaelea lauricola on camphor in Florida and Georgia. Plant Disease 2009, 93 (2), 198.

58. Hughes, M.; Smith, J. A.; Mayfield Iii, A. E.; Minno, M. C.; Shin, K. First Report of Laurel Wilt Disease Caused by Raffaelea lauricola on Pondspice in Florida. Plant Disease 2011, 95 (12), 1588.

59. Hughes, M. A.; Shin, K.; Eickwort, J. M.; Smith, J. A. First Report of Laurel Wilt Disease Caused by Raffaelea lauricola on Silk Bay in Florida. Plant Disease 2012, $96(6), 910$.

60. Hughes, M. A.; Black, A.; Smith, J. A. First Report of Laurel Wilt Caused by Raffaelea lauricola on Bay Laurel (Laurus nobilis) in the United States. Plant Disease 2014, 98 (8), 1159.

61. Chupp, A. D.; Battaglia, L. L. Potential for host shifting in Papilio palamedes following invasion of laurel wilt disease. Biological Invasions 2014, 16 (12), 2639-2651.

62. Lederhouse, R. C.; Ayres, M. P.; Nitao, J. K.; Scriber, J. M. Differential use of lauraceous hosts by swallowtail butterflies, Papilio troilus and P. palamedes (Papilionidae). Oikos 1992, 244-252.

63. Evans, J. P.; Scheffers, B. R.; Hess, M. Effect of laurel wilt invasion on redbay populations in a maritime forest community. Biological Invasions 2014, 16 (8), 1581-1588.

64. Davis, D.; Wagner, D. Biology and systematics of the New World Phyllocnistis Zeller leafminers of the avocado genus Persea (Lepidoptera, Gracillariidae).

ZooKeys 2011, 97, 39.

65. Wetzel, P. R.; van der Valk, A. G.; Newman, S.; Gawlik, D. E.; Troxler Gann, T.; Coronado-Molina, C. A.; Childers, D. L.; Sklar, F. H. Maintaining tree islands in the Florida Everglades: nutrient redistribution is the key. Frontiers in Ecology and the Environment 2005, 3 (7), 370-376.

66. Cahoon, D. R.; Hensel, P.; Rybczyk, J.; McKee, K. L.; Proffitt, C. E.; Perez, B. C. Mass tree mortality leads to mangrove peat collapse at Bay Islands, Honduras after Hurricane Mitch. Journal of Ecology 2003, 91 (6), 1093-1105.

67. Snyder, J. R. Ecological implications of laurel wilt infestation on Everglades tree islands, southern Florida; US Geological Survey: 14.

68. Brendemuehl, R. H. Persea borbonia (L.) Spreng. redbay. In Silvics of North America, Agricultural Handbook 654, Burns, R. M., Honkala, B. H., Eds.; Forest 
Service, United States Department of Agriculture: Washington, DC, 1990; Vol. 2, pp 503-506.

69. Korn, M. The Staggering Amounts of Food Eaten on Super Bowl Sunday. ABC News . 2-2-2017.

70. Gallagher, P. Redbay trees are dying. The Seminole Tribune, Feb 28, 14 A.D.

71. Snow, A. M.; Stans, S. E. Healing plants: medicine of the Florida Seminole Indians; University Press of Florida: 2001.

72. Bennett, B. C. An introduction to the Seminole people of south Florida and their plants. Part I: History and ethnology. The Palmetto Summer/Fall 1997, 20-21.

73. De Castro, A. I.; Ehsani, R.; Ploetz, R.; Crane, J. H.; Abdulridha, J. Optimum spectral and geometric parameters for early detection of laurel wilt disease in avocado. Remote Sensing of Environment 2015, 171, 33-44.

74. De Castro, A. I.; Ehsani, R.; Abdulridha, J.; Buchanon, S. M.; Ploetz, R. Classification of Laurel wilt-infested Avocado Trees. American Society of Agricultural and Biological Engineers: 2015; p 1.

75. De Castro, A. I.; Ehsani, R.; Ploetz, R. C.; Crane, J. H.; Buchanon, S. Detection of laurel wilt disease in avocado using low altitude aerial imaging. PloS one 2015, 10 (4), $\mathrm{e} 0124642$.

76. Sankaran, S.; Ehsani, R.; Inch, S. A.; Ploetz, R. C. Evaluation of visible-near infrared reflectance spectra of avocado leaves as a non-destructive sensing tool for detection of laurel wilt. Plant Disease 2012, 96 (11), 1683-1689.

77. Abdulridha, J.; Ehsani, R.; de Castro, A. Detection and Differentiation between Laurel Wilt Disease, Phytophthora Disease, and Salinity Damage Using a Hyperspectral Sensing Technique. Agriculture 2016, 6 (4), 56.

78. Pero, J. Bug Off My Guacamole. Mechanical Engineering Magazine . 2015.

79. Pulido, C. A.; Gebelein, J. L.; Mills, D. K. Use of multispectral imagery acquired by hand-held and UAV-mounted cameras: Differences between stressed avocado trees infected by Raffaelea lauricola and non-stressed trees. Unpublished Work, 2017.

80. Hill, S.; Hill, J. Richard Henry of Resolution Island; J. McIndoe: 1987.

81. Zwickel, F. C. Use of dogs in wildlife management. Wildlife Management Techniques 1971, 319-324. 
82. Ensminger, J. Canine Biodetection in Conservation, Eradication, and Border Protection: A Regulatory Perspective. In Canine Olfaction Science and Law: Advances in Forensic Science, Medicine, Conservation, and Environmental Remediation, CRC Press: 2016; pp 351-370.

83. Hurt, A.; Smith, D. A. Conservation dogs. Canine ergonomics: the science of working dogs. CRC Press, Boca Raton, Florida, USA 2009, 175-194.

84. Springer, K. Detection Dogs in Strategies for Eradicating Pest Species from Natural Environments. In Canine Olfaction Science and Law: Advances in Forensic Science, Medicine, Conservation, and Environmental Remediation, CRC Press: 2016; pp 335-349.

85. Beebe, S. C.; Howell, T. J.; Bennett, P. C. Using Scent Detection Dogs in Conservation Settings: A Review of Scientific Literature Regarding Their Selection. Frontiers in Veterinary Science 2016, 3.

86. Mills, D. K. The Science of Laurel Wilt Canine Detection. 2017.

87. Goldblatt, A.; Gazit, I.; Terkel, J. Olfaction and explosives detector dogs. Canine Ergonomics: The Science of Working Dogs 2009, 135-174.

88. Wewetzer, K.; Omar, M.; Kammeyer, P.; Brandes, G. Wiring of the Olfactory System and the Functional Role of Neurons and Glia during Lifelong Turnover. In Canine Olfaction Science and Law: Advances in Forensic Science, Medicine, Conservation, and Environmental Remediation, CRC Press: 2016; pp 17-29.

89. Buck, L. B.; Bargmann, C. Smell and taste: The chemical senses. Principles of neural science 2000, 4, 625-647.

90. Quignon, P.; Kirkness, E.; Cadieu, E.; Touleimat, N.; Guyon, R.; Renier, C.; Hitte, C.; André, C.; Fraser, C.; Galibert, F. Comparison of the canine and human olfactory receptor gene repertoires. Genome biology 2003, 4 (12), R80.

91. Craven, B. A.; Neuberger, T.; Paterson, E. G.; Webb, A. G.; Josephson, E. M.; Morrison, E. E.; Settles, G. S. Reconstruction and morphometric analysis of the nasal airway of the dog (Canis familiaris) and implications regarding olfactory airflow. The Anatomical Record 2007, 290 (11), 1325-1340.

92. Craven, B. A.; Paterson, E. G.; Settles, G. S. The fluid dynamics of canine olfaction: unique nasal airflow patterns as an explanation of macrosmia. Journal of The Royal Society Interface 2009, rsif20090490.

93. Steen, J. B.; Mohus, I.; Kvesetberg, T.; Walloe, L. Olfaction in bird dogs during hunting. Acta physiologica scandinavica 1996, 157 (1), 115-119. 
94. Moore, P. A. Aerodynamics of Odor Plumes and Odor Plume Structures in Different Habitats. In Canine Olfaction Science and Law: Advances in Forensic Science, Medicine, Conservation, and Environmental Remediation, CRC Press: 2016; pp 87-102.

95. Hall, N. J.; Wynne, C. D. Canine Olfactory Learning and Behavior. In Canine Olfaction Science and Law: Advances in Forensic Science, Medicine, Conservation, and Environmental Remediation, CRC Press: 2016; pp 123-137.

96. Hurt, A.; Woollett, D. A. S.; Parker, M. Training Considerationsin Wildlife Detection. In Canine Olfaction Science and Law: Advances in Forensic Science, Medicine, Conservation, and Environmental Remediation, CRC Press: 2016; pp 139-153.

97. Lehnert, M. P.; Weeks, E. N. Trained Dogs in Insect Detection. In Canine Olfaction Science and Law: Advances in Forensic Science, Medicine, Conservation, and Environmental Remediation, CRC Press: 2016; pp 321-333.

98. Harper, R. J.; Almirall, J. R.; Furton, K. G. Identification of dominant odor chemicals emanating from explosives for use in developing optimal training aid combinations and mimics for canine detection. Talanta 2005, 67 (2), 313-327.

99. Papet, L. E. Narcotic and Explosive Odors: Volatile Organic Compounds as Training Aids for Olfactory Detection. In Canine Olfaction Science and Law: Advances in Forensic Science, Medicine, Conservation, and Environmental Remediation, CRC Press: 2016; pp 265-278.

100. Furton, K. G.; Myers, L. J. The scientific foundation and efficacy of the use of canines as chemical detectors for explosives. Talanta 2001, 54 (3), 487-500.

101. Furton, K. G.; Caraballo, N. I.; Cerreta, M. M.; Holness, H. K. Advances in the use of odour as forensic evidence through optimizing and standardizing instruments and canines. Phil. Trans. R. Soc. B 2015, 370 (1674), 20140262.

102. Harper, R. J.; Furton, K. G. Biological detection of explosives. Counterterrorist detection techniques of explosives. Amsterdam, The Netherlands 2007, 395-431.

103. Lorenzo, N.; Wan, T.; Harper, R. J.; Hsu, Y. L.; Chow, M.; Rose, S.; Furton, K. G. Laboratory and field experiments used to identify Canis lupus var. familiaris active odor signature chemicals from drugs, explosives, and humans. Analytical and bioanalytical chemistry 2003, 376 (8), 1212-1224.

104. Rudnicka, J.; Buszewski, B. The Development of Gas Chromatography/Mass Spectrometry and Its Uses in Odor Analysis. In Canine Olfaction Science and Law: Advances in Forensic Science, Medicine, Conservation, and Environmental Remediation, CRC Press: 2016; pp 71-85. 
105. Furton, K. G.; Harper, R. J. Controlled Odor Mimic Permeation System. 7-182007. Google Patents.

106. Crane, J. H.; Carrillo, D.; Ploetz, R. C.; Evans, E. A.; Palmateer, A. J.; Pybas, D. Current status and control recommendations for laurel wilt and the ambrosia beetle vectors in commercial avocado (Persea americana L.) orchards in south Florida. 2015; pp 240-244.

107. Ploetz, R. C.; Pérez-Martínez, J. M.; Evans, E. A.; Inch, S. A. Toward fungicidal management of laurel wilt of avocado. Plant Disease 2011, 95 (8), 977-982.

108. Brar, G. S.; Capinera, J. L.; Kendra, P. E.; McLean, S.; Peña, J. E. Life cycle, development, and culture of Xyleborus glabratus (Coleoptera: Curculionidae: Scolytinae). Florida Entomologist 2013, 96 (3), 1158-1167.

109. Peña, J. E.; Crane, J. H.; Capinera, J. L.; Duncan, R. E.; Kendra, P. E.; Ploetz, R. C.; McLean, S.; Brar, G.; Thomas, M. C.; Cave, R. D. Chemical control of the redbay ambrosia beetle, Xyleborus glabratus, and other Scolytinae (Coleoptera: Curculionidae). Florida Entomologist 2011, 94 (4), 882-896.

110. Carrillo, D.; Crane, J. H.; Peña, J. E. Potential of contact insecticides to control Xyleborus glabratus (Coleoptera: Curculionidae), a vector of laurel wilt disease in avocados. Journal of economic entomology 2013, 106 (6), 2286-2295.

111. Brar, G. S.; Capinera, J. L.; McLean, S.; Kendra, P. E.; Ploetz, R. C.; Peña, J. E. Effect of trap size, trap height and age of lure on sampling Xyleborus glabratus (Coleoptera: Curculionidae: Scolytinae), and its flight periodicity and seasonality. Florida Entomologist 2012, 95 (4), 1003-1011.

112. Hanula, J. L.; Sullivan, B. Manuka oil and phoebe oil are attractive baits for Xyleborus glabratus (Coleoptera: Scolytinae), the vector of laurel wilt. Environmental entomology 2008, 37 (6), 1403-1409.

113. Hanula, J. L.; Ulyshen, M. D.; Horn, S. Effect of trap type, trap position, time of year, and beetle density on captures of the redbay ambrosia beetle (Coleoptera: Curculionidae: Scolytinae). Journal of economic entomology 2011, 104 (2), 501508.

114. Hanula, J. L.; Sullivan, B. T.; Wakarchuk, D. Variation in manuka oil lure efficacy for capturing Xyleborus glabratus (Coleoptera: Curculionidae: Scolytinae), and cubeb oil as an alternative attractant. Environmental entomology 2013, 42 (2), 333-340.

115. Hanula, J. L.; Mayfield, A. E.; Reid, L. S.; Horn, S. Influence of trap distance from a source population and multiple traps on captures and attack densities of the 
redbay ambrosia beetle (Coleoptera: Curculionidae: Scolytinae). Journal of economic entomology 2016, tow068.

116. Kendra, P. E.; Montgomery, W. S.; Niogret, J.; Peña, J. E.; Capinera, J. L.; Brar, G.; Epsky, N. D.; Heath, R. R. Attraction of the redbay ambrosia beetle, Xyleborus glabratus, to avocado, lychee, and essential oil lures. Journal of chemical ecology 2011, 37 (9), 932.

117. Kendra, P. E.; Niogret, J.; Montgomery, W. S.; Sanchez, J. S.; Deyrup, M. A.; Pruett, G. E.; Ploetz, R. C.; Epsky, N. D.; Heath, R. R. Temporal analysis of sesquiterpene emissions from manuka and phoebe oil lures and efficacy for attraction of Xyleborus glabratus (Coleoptera: Curculionidae: Scolytinae). Journal of economic entomology 2012, 105 (2), 659-669.

118. Kendra, P. E.; Montgomery, W. S.; Niogret, J.; Schnell, E. Q.; Deyrup, M. A.; Epsky, N. D. Evaluation of seven essential oils identifies cubeb oil as most effective attractant for detection of Xyleborus glabratus. Journal of Pest Science 2014, 87 (4), 681-689.

119. Kendra, P. E.; Montgomery, W. S.; Deyrup, M. A.; Wakarchuk, D. Improved lure for redbay ambrosia beetle developed by enrichment of .alpha.-copaene content. Journal of Pest Science 2016, 89 (2), 427-438.

120. Kendra, P. E.; Montgomery, W. S.; Niogret, J.; Epsky, N. D. An uncertain future for American Lauraceae: A lethal threat from redbay ambrosia beetle and laurel wilt disease (A review). 2013.

121. Kuhns, E. H.; Martini, X.; Tribuiani, Y.; Coy, M.; Gibbard, C.; Peña, J.; Hulcr, J.; Stelinski, L. L. Eucalyptol is an attractant of the redbay ambrosia beetle, Xyleborus glabratus. Journal of chemical ecology 2014, 40 (4), 355-362.

122. Kendra, P. E.; Niogret, J.; Montgomery, W. S.; Deyrup, M. A.; Epsky, N. D. Cubeb oil lures: terpenoid emissions, trapping efficacy, and longevity for attraction of redbay ambrosia beetle (Coleoptera: Curculionidae: Scolytinae). Journal of economic entomology 2015, 108 (1), 350-361.

123. Hughes, M. A.; Martini, X.; Kuhns, E.; Colee, J.; Mafra-Neto, A.; Stelinski, L. L.; Smith, J. A. Evaluation of repellents for the redbay ambrosia beetle, Xyleborus glabratus, vector of the laurel wilt pathogen. Journal of Applied Entomology 2017.

124. Wercinski, S. A. Solid phase microextraction: a practical guide, CRC Press: 1999. 
125. MacCrehan, W.; Moore, S.; Schantz, M. Evaluating headspace component vaportime profiles by solid-phase microextraction with external sampling of an internal standard. Analytical chemistry 2011, 83 (22), 8560-8565.

126. MacCrehan, W.; Moore, S.; Schantz, M. Reproducible vapor-time profiles using solid-phase microextraction with an externally sampled internal standard. Journal of Chromatography A 2012, 1244, 28-36.

127. Mochalski, P.; Wzorek, B.; Sliwka, I.; Amann, A. Suitability of different polymer bags for storage of volatile sulphur compounds relevant to breath analysis. Journal of Chromatography B 2009, 877 (3), 189-196.

128. Handley, A. J.; Adlard, E. R. Gas chromatographic techniques and applications; 5 ed.; Taylor \& Francis: 2001.

129. Harris, D. C. Exploring chemical analysis; Macmillan: 2012.

130. Rouessac, F.; Rouessac, A. Chemical analysis: modern instrumentation methods and techniques; John Wiley \& Sons: 2013.

131. De Hoffmann, E.; Stroobant, V. Mass spectrometry: principles and applications; John Wiley \& Sons: 2007.

132. Todokoro, H.; Ezumi, M. Scanning electron microscope. 2-16-1999. Google Patents.

133. Exley, R. R.; Butterfield, B. G.; Meylan, B. A. Preparation of wood specimens for the scanning electron microscope. Journal of microscopy 1974, 101 (1), 21-30.

134. Inch, S.; Ploetz, R.; Held, B.; Blanchette, R. Histological and anatomical responses in avocado, Persea americana, induced by the vascular wilt pathogen, Raffaelea lauricola. Botany 2012, 90 (7), 627-635.

135. Millar, J. N.; Millar, J. C. Statistics and chemometrics for analytical chemistry. UK: Prentice Hall 2000.

136. Lavine, B. K. Chemometrics and chemoinformatics; ACS Publications: 2005.

137. Gowda, H.; Ivanisevic, J.; Johnson, C. H.; Kurczy, M. E.; Benton, H. P.; Rinehart, D.; Nguyen, T.; Ray, J.; Kuehl, J.; Arevalo, B. Interactive XCMS Online: simplifying advanced metabolomic data processing and subsequent statistical analyses. Analytical chemistry 2014, 86 (14), 6931-6939.

138. Helton, W. S. Overview of scent detection work. Canine Ergonomics: The Science of Working Dogs 2009, 83. 
139. DeGreeff, L. E.; Weakley-Jones, B.; Furton, K. G. Creation of training aids for human remains detection canines utilizing a non-contact, dynamic airflow volatile concentration technique. Forensic science international 2012, 217 (1), 32-38.

140. Griffith, R. T.; Jayachandran, K.; Whitstine, W.; Furton, K. G. Differentiation of toxic molds via headspace SPME-GC/MS and canine detection. Sensors 2007, 7 (8), 1496-1508.

141. Crane, J. H.; Carrillo, D.; Ploetz, R. C.; Evans, E. A.; Wasielewski, J. Recommendations for Ambrosia Beetle and Laurel Wilt Pathogen Control. 2015.

142. Furton, K.; Greb, J.; Holness, H. The scientific working group on dog and orthogonal detector guidelines (SWGDOG). National Criminal Justive Reference Service 2010, 155.

143. Furton, K. G.; Beltz, K. Universal detector calibrant. 2-21-2017. Google Patents.

144. Agrell, J.; McDonald, E. P.; Lindroth, R. L. Effects of CO2 and light on tree phytochemistry and insect performance. Oikos 2000, 88 (2), 259-272.

145. Berenbaum, M. R.; Zangerl, A. R. Genetics of secondary metabolism and herbivore resistance in plants. Herbivores: their interactions with secondary plant metabolites 1992, 2, 415-438.

146. Bowers, M. D.; Stamp, N. E. Effects of plant age, genotype and herbivory on Plantago performance and chemistry. Ecology 1993, 74 (6), 1778-1791.

147. Chen, X. Y.; Chen, Y.; Heinstein, P.; Davisson, V. J. Cloning, expression, and characterization of $(+)$-d-cadinene synthase: a catalyst for cotton phytoalexin biosynthesis. Archives of Biochemistry and Biophysics 1995, 324 (2), 255-266.

148. Pirrung, M. C.; Morehead Jr, A. T.; Young, B. G.; Goldsmith, D. The Total Synthesis of Natural Products, Volume 11, Part B: Bicyclic and Tricyclic Sesquiterpenes; 11 ed.; John Wiley \& Sons: 2009.

149. Langenheim, J. H. Higher plant terpenoids: a phytocentric overview of their ecological roles. Journal of chemical ecology 1994, 20 (6), 1223-1280.

150. Kumeta, Y.; Ito, M. Characterization of +-'-guaiene synthases from cultured cells of Aquilaria, responsible for the formation of the sesquiterpenes in agarwood. Plant physiology 2010, 154 (4), 1998-2007.

151. Kuhns, E. H.; Martini, X.; Tribuiani, Y.; Coy, M.; Gibbard, C.; Peña, J.; Hulcr, J.; Stelinski, L. L. Eucalyptol is an attractant of the redbay ambrosia beetle, Xyleborus glabratus. Journal of chemical ecology 2014, 40 (4), 355-362. 
152. Macias, M. S.; Furton, K. G. Availability of target odor compounds from seized ecstasy tablets for canine detection. Journal of forensic sciences 2011, 56 (6), 1594-1600. 


\section{APPENDIX}

The appendix contains information related to the experiments and results presented in the dissertation.

Table 17: Location and relevant weather data for samples collected by the authors. Samples missing data were collected either by farmers or by canine handlers following the sampling protocols.

\begin{tabular}{|c|c|c|c|c|c|c|}
\hline & Sample No. & Collection Date & Time & Temperature $\left({ }^{\circ} \mathrm{F}\right)$ & Humidity (\%) & Dew Point $\left({ }^{\circ}\right)$ \\
\hline \multirow{15}{*}{$\begin{array}{l}\text { Infected } \\
\text { Samples }\end{array}$} & 1 & $8 / 4 / 2014$ & $3: 30$ & - & - & - \\
\hline & 2 & $8 / 4 / 2014$ & $15: 30$ & - & - & - \\
\hline & 3 & $10 / 6 / 2014$ & $17: 30$ & - & - & - \\
\hline & 4 & $10 / 6 / 2014$ & $17: 30$ & - & - & - \\
\hline & 5 & $10 / 6 / 2014$ & $17: 30$ & - & - & - \\
\hline & 6 & $1 / 20 / 15$ & $7: 45$ & 64 & 84 & 59 \\
\hline & 7 & $2 / 5 / 2015$ & $6: 45$ & 72 & 88 & 68 \\
\hline & 8 & $3 / 31 / 2015$ & - & - & - & - \\
\hline & 9 & $4 / 7 / 2015$ & - & - & - & - \\
\hline & 10 & $5 / 4 / 2015$ & $8: 00$ & 72 & 70 & 62 \\
\hline & 11 & $7 / 1 / 2015$ & $11: 45$ & 89 & 62 & 74 \\
\hline & 12 & $6 / 19 / 2015$ & $9: 50$ & 85 & 79 & 76 \\
\hline & 13 & $9 / 12 / 2015$ & $9: 30$ & 85 & 76 & 77 \\
\hline & 14 & $9 / 12 / 2015$ & 9:30 & 85 & 76 & 77 \\
\hline & 15 & $9 / 12 / 2015$ & 9:30 & 85 & 76 & 77 \\
\hline \multirow{9}{*}{$\begin{array}{l}\text { Healthy } \\
\text { Samples }\end{array}$} & 16 & $8 / 4 / 2014$ & $15: 30$ & - & - & - \\
\hline & 17 & $10 / 6 / 2014$ & $17: 30$ & - & - & - \\
\hline & 18 & $10 / 6 / 2014$ & $17: 30$ & - & - & - \\
\hline & 19 & $1 / 5 / 2015$ & $11: 00$ & 79 & 71 & 71 \\
\hline & 20 & 4/7/2015 & - & - & - & - \\
\hline & 21 & $8 / 12 / 2015$ & $12: 00$ & 88 & 58 & 73 \\
\hline & 22 & $9 / 12 / 2015$ & $10: 00$ & 85 & 76 & 77 \\
\hline & 23 & $9 / 12 / 2015$ & $10: 00$ & 85 & 76 & 77 \\
\hline & 24 & $9 / 12 / 2015$ & $10: 00$ & 85 & 76 & 77 \\
\hline
\end{tabular}


Table 18: Specified laboratory parameters from Task 4 greenhouse experiments of avocado trees inoculated with $R$. lauricola. Parameters were recorded at the beginning of SPME fiber exposure. (Continued next page)

\begin{tabular}{|c|c|c|c|}
\hline Temperature $\left({ }^{\circ} \mathrm{C}\right)$ & Humidity (\%) & Sample Collection Day & Sample Tree \\
\hline 19.9 & 46 & DAY 1 & $1,2,3,4,5,6$ \\
\hline 20.2 & 46 & DAY 1 & $7,8,9,10$, BLANKS \\
\hline $20.6-22.9$ & $42-61$ & DAY 3 & 2-10, BLANKS \\
\hline 20.6 & 45 & DAY 3 & 1 \\
\hline 20.6 & 43 & DAY 6 & $6,8,9$ \\
\hline 20.4 & 43 & DAY 6 & $4,7,10$ \\
\hline 20.6 & 42 & DAY 6 & $2,5,1 \mathrm{~A}, 1 \mathrm{C}$ \\
\hline 20.6 & 44 & DAY 6 & 3, BLANKS \\
\hline 20.9 & 42 & DAY 9 & $6,7,9$ \\
\hline 20.4 & 45 & DAY 9 & $5,8,10$ \\
\hline 20.4 & 45 & DAY 9 & 4 \\
\hline 20.3 & 45 & DAY 9 & 2,3 \\
\hline 20.4 & 44 & DAY 9 & 1, BLANKS \\
\hline 20.5 & 46 & DAY 12 & $8,9,10$ \\
\hline 20.1 & 44 & DAY 12 & $4,6,7$ \\
\hline 19.9 & 51 & DAY 12 & $1,3,5$ \\
\hline 20.3 & 43 & DAY 12 & 2, BLANKS \\
\hline 19.9 & 52 & DAY 15 & $4,6,7$ \\
\hline 20.1 & 42 & DAY 15 & $5,9,10$ \\
\hline 20.4 & 44 & DAY 15 & $1,2,8$ \\
\hline 20.1 & 43 & DAY 15 & 3, BLANKS \\
\hline 20.1 & 43 & DAY 18 & $6,7,10$ \\
\hline 20.4 & 43 & DAY 18 & $4,8,9$ \\
\hline 20.1 & 46 & DAY 18 & $2,3,5$ \\
\hline 20.5 & 44 & DAY 18 & 1, BLANKS \\
\hline 20.4 & 44 & DAY 21 & $6,10,9 B, 9 C$ \\
\hline 20.4 & 42 & DAY 21 & $4,7,8,9 A$ \\
\hline 19.9 & 45 & DAY 21 & $2,5,1 \mathrm{~B}, 1 \mathrm{C}$ \\
\hline 20.0 & 40 & DAY 21 & 3, BLANKS \\
\hline 20.4 & 42 & DAY 24 & $3,4,5$ \\
\hline 20.5 & 41 & DAY 24 & $1,2,9$ \\
\hline 20.0 & 45 & DAY 24 & 7,10 \\
\hline 19.9 & 46 & DAY 24 & 6,8, BLANKS \\
\hline 19.4 & 41 & DAY 27 & 9,10 \\
\hline 19.4 & 40 & DAY 27 & $6,7,8$ \\
\hline
\end{tabular}




\begin{tabular}{|c|c|c|c|}
\hline Temperature $\left({ }^{\circ} \mathrm{C}\right)$ & Humidity (\%) & Sample Collection Day & Sample Tree \\
\hline 19.9 & 50 & DAY 27 & $2,3,5$ \\
\hline 20.0 & 44 & DAY 27 & 1,4, BLANKS \\
\hline 20.4 & 45 & DAY 30 & $6,9,10$ \\
\hline 20.9 & 42 & DAY 30 & $5,7,8$, BLANK O \\
\hline 20.9 & 40 & DAY 30 & 2,3 \\
\hline 20.9 & 40 & DAY 30 & 1, BLANK G \\
\hline 20.9 & 41 & DAY 30 & 4 \\
\hline 20.4 & 42 & DAY 33 & $6,9,10$ \\
\hline 21.4 & 44 & DAY 33 & $1,7,8$ \\
\hline 21.1 & 47 & DAY 33 & $2,3,5$ \\
\hline 21.9 & 48 & DAY 33 & BLANK O \\
\hline 22.0 & 47 & DAY 33 & 4, BLANK G \\
\hline 21.9 & 50 & DAY 36 & $6,7,10$ \\
\hline 21.9 & 54 & DAY 36 & $5,8,9$ \\
\hline 23.1 & 52 & DAY 36 & $2,3,4$ \\
\hline 23.6 & 50 & DAY 36 & 1, BLANKS \\
\hline 22.4 & 47 & DAY 39 & $6,7,10$ \\
\hline 20.6 & 44 & DAY 39 & 8, BLANK 0 \\
\hline 21.4 & 51 & DAY 39 & $4,5,9$ \\
\hline 22.1 & 49 & DAY 39 & $1,2,3$ \\
\hline 22.6 & 50 & DAY 39 & BLANK G \\
\hline 22.4 & 52 & DAY 42 & 6,10 \\
\hline 22.9 & 49 & DAY 42 & 7, BLANK 0 \\
\hline 22.5 & 50 & DAY 42 & $5,8,9$ \\
\hline 22 & 50 & DAY 42 & $1,2,3$ \\
\hline 22.8 & 47 & DAY 42 & 4, BLANK G \\
\hline 19.6 & 53 & DAY 48 & 6,7, BLANK O \\
\hline 20.6 & 47 & DAY 48 & 8,9 \\
\hline 20.9 & 46 & DAY 48 & $10,1 C$ \\
\hline 21 & 45 & DAY 48 & 2 \\
\hline 21.4 & 49 & DAY 48 & 1A,1B, BLANK G \\
\hline 20.1 & 47 & DAY 51 & $6,7,8$ \\
\hline 19.6 & 50 & DAY 51 & $9,10,2$ \\
\hline 21.3 & 48 & DAY 51 & $3,4,1 \mathrm{C}$, BLANK G \\
\hline 21.5 & 49 & DAY 51 & 5 \\
\hline 21.9 & 48 & DAY 51 & 1A,1B, BLANK O \\
\hline
\end{tabular}


Table 19: Specified laboratory parameters from Task 4 greenhouse experiments of avocado trees inoculated with $R$. lauricola and then treated with propiconazole. Parameters were recorded at the beginning of SPME fiber exposure. (Continued next two pages)

\begin{tabular}{|c|c|c|c|}
\hline Temperature $\left({ }^{\circ} \mathrm{C}\right)$ & Humidity (\%) & Sample Collection Day & Sample Tree \\
\hline 16.4 & 46 & DAY 0 & 15,16 \\
\hline 16.6 & 46 & DAY 0 & 11,17 \\
\hline 16.6 & 45 & DAY 0 & 12,13 \\
\hline 16.1 & 47 & DAY 0 & 14,18 \\
\hline 16.9 & 48 & DAY 0 & 19,20 \\
\hline 17.4 & 49 & DAY 0 & 21, BLANKS \\
\hline 18.0 & 49 & DAY 1 & 15,16 \\
\hline 18.4 & 54 & DAY 1 & 11,17 \\
\hline 19.0 & 52 & DAY 1 & 12,13 \\
\hline 19.4 & 52 & DAY 1 & 14,18 \\
\hline 19.4 & 52 & DAY 1 & 19,20 \\
\hline 18.6 & 52 & DAY 1 & 21, BLANKS \\
\hline 18.6 & 52 & DAY 3 & 15,16 \\
\hline 19.4 & 51 & DAY 3 & 11,17 \\
\hline 19.4 & 51 & DAY 3 & 12,13 \\
\hline 18.9 & 52 & DAY 3 & 14,18 \\
\hline 19.4 & 51 & DAY 3 & 19,20 \\
\hline 19.6 & 50 & DAY 3 & 21 \\
\hline 19.5 & 50 & DAY 3 & BLANKS \\
\hline 19.6 & 50 & DAY 5 & 15,16 \\
\hline 19.9 & 50 & DAY 5 & 11,17 \\
\hline 19.6 & 52 & DAY 5 & 12,13 \\
\hline 20.1 & 43 & DAY 5 & 14,18 \\
\hline 20.1 & 43 & DAY 5 & 19,20 \\
\hline 20.3 & 43 & DAY 5 & 21, BLANKS \\
\hline 20.4 & 44 & DAY 7 & 15,16 \\
\hline 20.1 & 46 & DAY 7 & 11,17 \\
\hline 20.4 & 44 & DAY 7 & 12,13 \\
\hline 20.4 & 42 & DAY 7 & 14,18 \\
\hline 20.1 & 43 & DAY 7 & 19,20 \\
\hline 20.1 & 42 & DAY 7 & 21 \\
\hline 20.3 & 43 & DAY 7 & BLANKS \\
\hline 20.3 & 45 & DAY 8 & 15,16 \\
\hline
\end{tabular}




\begin{tabular}{|c|c|c|c|}
\hline Temperature $\left({ }^{\circ} \mathrm{C}\right)$ & Humidity (\%) & Sample Collection Day & Sample Tree \\
\hline 20.3 & 43 & DAY 8 & 11,17 \\
\hline 20.4 & 43 & DAY 8 & 12,13 \\
\hline 20.4 & 44 & DAY 8 & 14 \\
\hline 18.6 & 43 & DAY 8 & 18 \\
\hline 19.3 & 45 & DAY 8 & 19,20 \\
\hline 19.4 & 44 & DAY 8 & 21, BLANKS \\
\hline 18.9 & 46 & DAY 10 & 15,16 \\
\hline 18.3 & 50 & DAY 10 & 11,17 \\
\hline 18.9 & 48 & DAY 10 & 12,13 \\
\hline 19.0 & 48 & DAY 10 & 14,18 \\
\hline 17.9 & 46 & DAY 10 & 19,20 \\
\hline 18.9 & 51 & DAY 10 & 21, BLANKS \\
\hline 19.4 & 50 & DAY 13 & $15,16,17$ \\
\hline 19.4 & 50 & DAY 13 & 11,12 \\
\hline 19.6 & 49 & DAY 13 & 13 \\
\hline 19.4 & 45 & DAY 13 & 20,21 \\
\hline 24.4 & 36 & DAY 13 & 14, BLANKS \\
\hline 20.5 & 41 & DAY 13 & 18,19 \\
\hline 20.4 & 42 & DAY 17 & 11,15 \\
\hline 20.4 & 43 & DAY 17 & $12,13,14,16$ \\
\hline 18.4 & 50 & DAY 17 & 18,19 \\
\hline 18.8 & 50 & DAY 17 & 20,21, BLANKS \\
\hline 18.6 & 53 & DAY 22 & 15,16 \\
\hline 19.1 & 55 & DAY 22 & 11,12 \\
\hline 18.9 & 52 & DAY 22 & 13,14 \\
\hline 19.1 & 52 & DAY 22 & 18,19 \\
\hline 19.1 & 51 & DAY 22 & BLANKS \\
\hline 19.3 & 51 & DAY 22 & 20,21 \\
\hline 19.4 & 51 & DAY 26 & 15,16 \\
\hline 19.3 & 52 & DAY 26 & 11,12 \\
\hline 19.6 & 50 & DAY 26 & 13,14 \\
\hline 19.8 & 50 & DAY 26 & 18,19 \\
\hline 19.6 & 51 & DAY 26 & 20,21 \\
\hline 19.9 & 52 & DAY 26 & BLANKS \\
\hline 19.9 & 51 & DAY 29 & 15,16 \\
\hline 19.4 & 52 & DAY 29 & 11,12 \\
\hline 17.4 & 44 & DAY 29 & 13,14 \\
\hline 17.9 & 43 & DAY 29 & 18,19 \\
\hline
\end{tabular}




\begin{tabular}{|c|c|c|c|}
\hline Temperature $\left({ }^{\circ} \mathbf{C}\right)$ & Humidity (\%) & Sample Collection Day & Sample Tree \\
\hline 17.9 & 43 & DAY 29 & $20 A, B$ \\
\hline 17.9 & 43 & DAY 29 & $21 \mathrm{~A}, \mathrm{~B}$ \\
\hline 19.6 & 52 & DAY 29 & $20 \mathrm{C}, 21 \mathrm{C}$, BLANKS \\
\hline 17.4 & 37 & DAY 32 & 18,19 \\
\hline 14.9 & 32 & DAY 32 & 20,21 \\
\hline 15.9 & 33 & DAY 32 & 11,12 \\
\hline 16.1 & 34 & DAY 32 & 15,16 \\
\hline 16.4 & 34 & DAY 32 & 13 \\
\hline 17.3 & 45 & DAY 32 & 14, BLANKS \\
\hline
\end{tabular}

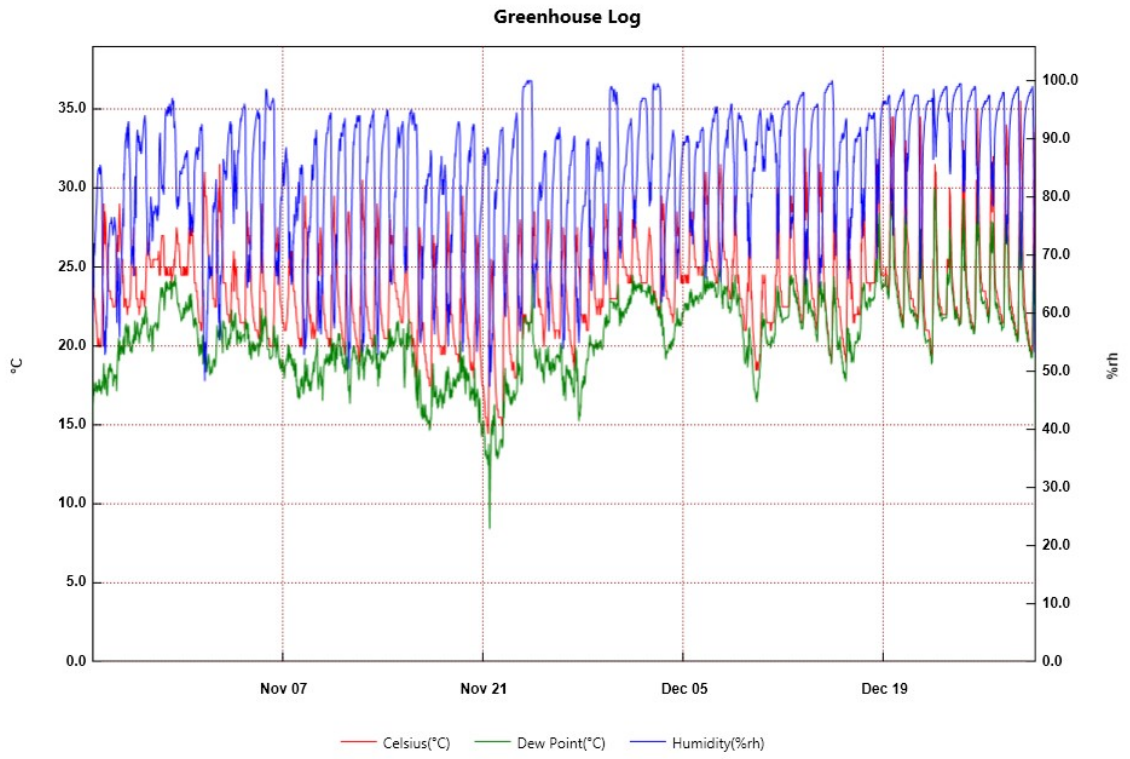

From: Monday, October 24, 2016 4:00:00 PM - To: Thursday, December 29, 2016 4:30:00 PM

Figure 62: Relevant weather data collected for trees located in the greenhouse for Task 4 greenhouse experiments of avocado trees inoculated with $R$. lauricola. 


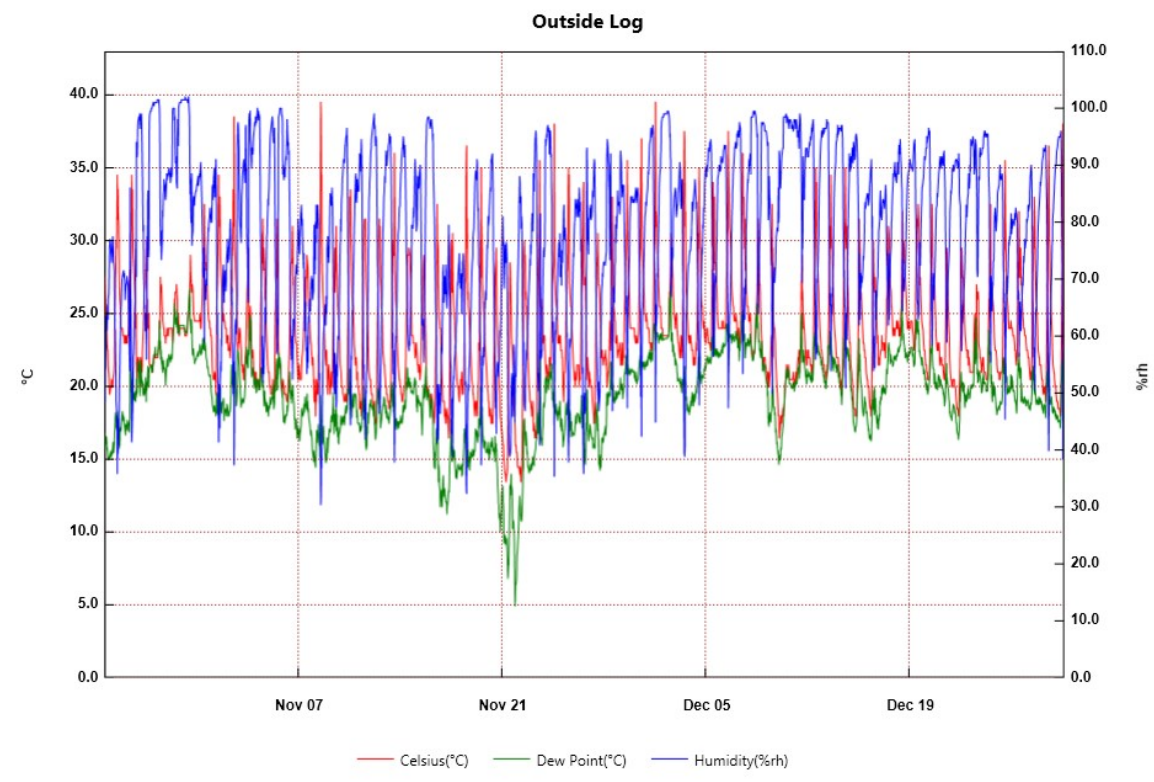

Figure 63: Relevant weather data collected for trees located outside for Task 4 greenhouse experiments of avocado trees inoculated with $\boldsymbol{R}$. lauricola.

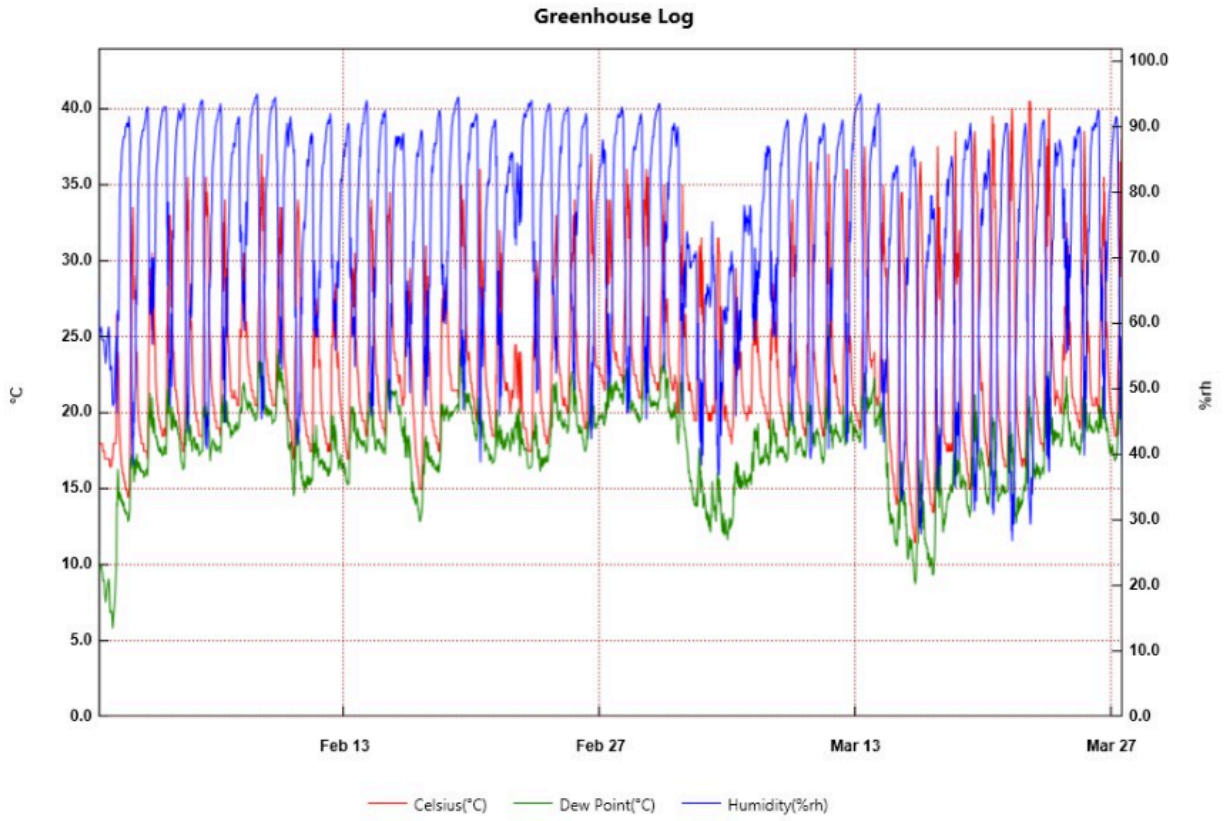

From: Monday, January 30, 2017 3:00:00 PM - To: Monday, March 27, 2017 3:00:00 PM

Figure 64: Relevant weather data collected for trees located in the greenhouse for Task 4 greenhouse experiments of avocado trees inoculated with $R$. lauricola and then treated with propiconazole. 


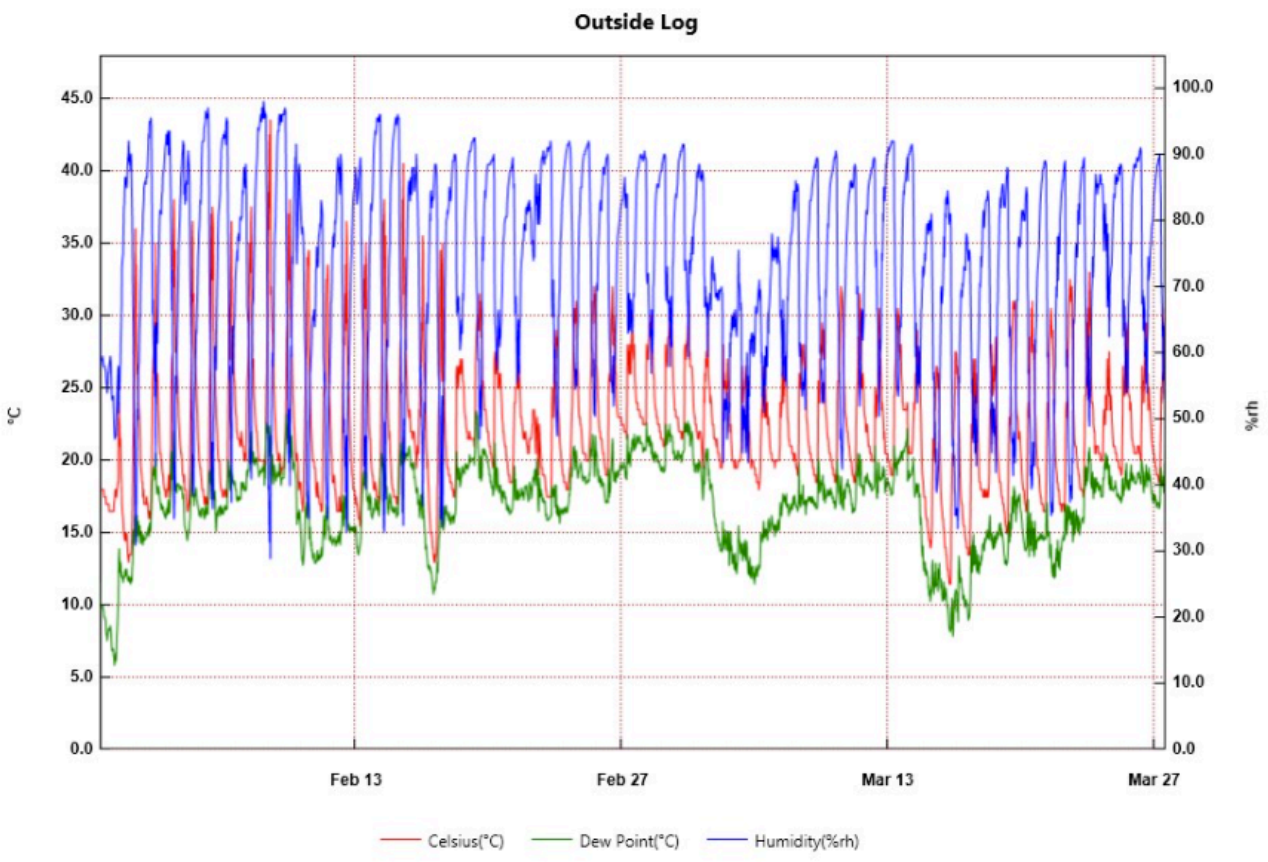

From: Monday, January 30, 2017 3:00:00 PM - To: Monday, March 27, 2017 3:00:00 PM

Figure 65: Relevant weather data collected for trees located outside for Task 4 greenhouse experiments of avocado trees inoculated with $\boldsymbol{R}$. lauricola and then treated with propiconazole.

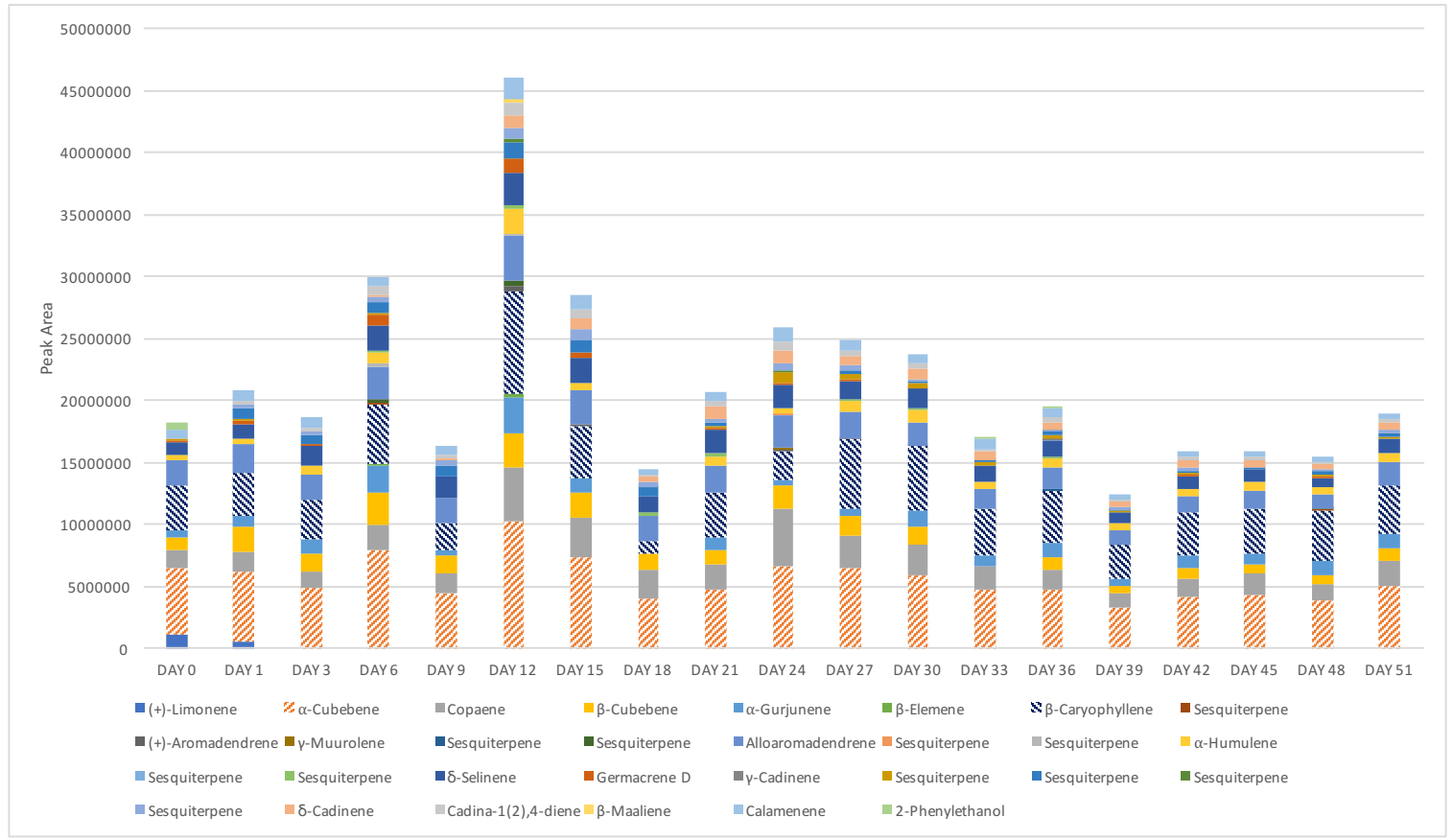

Figure 66: Odor profile of avocado trees inoculated with $\boldsymbol{R}$. lauricola by sample day for Section 5.4.1. 


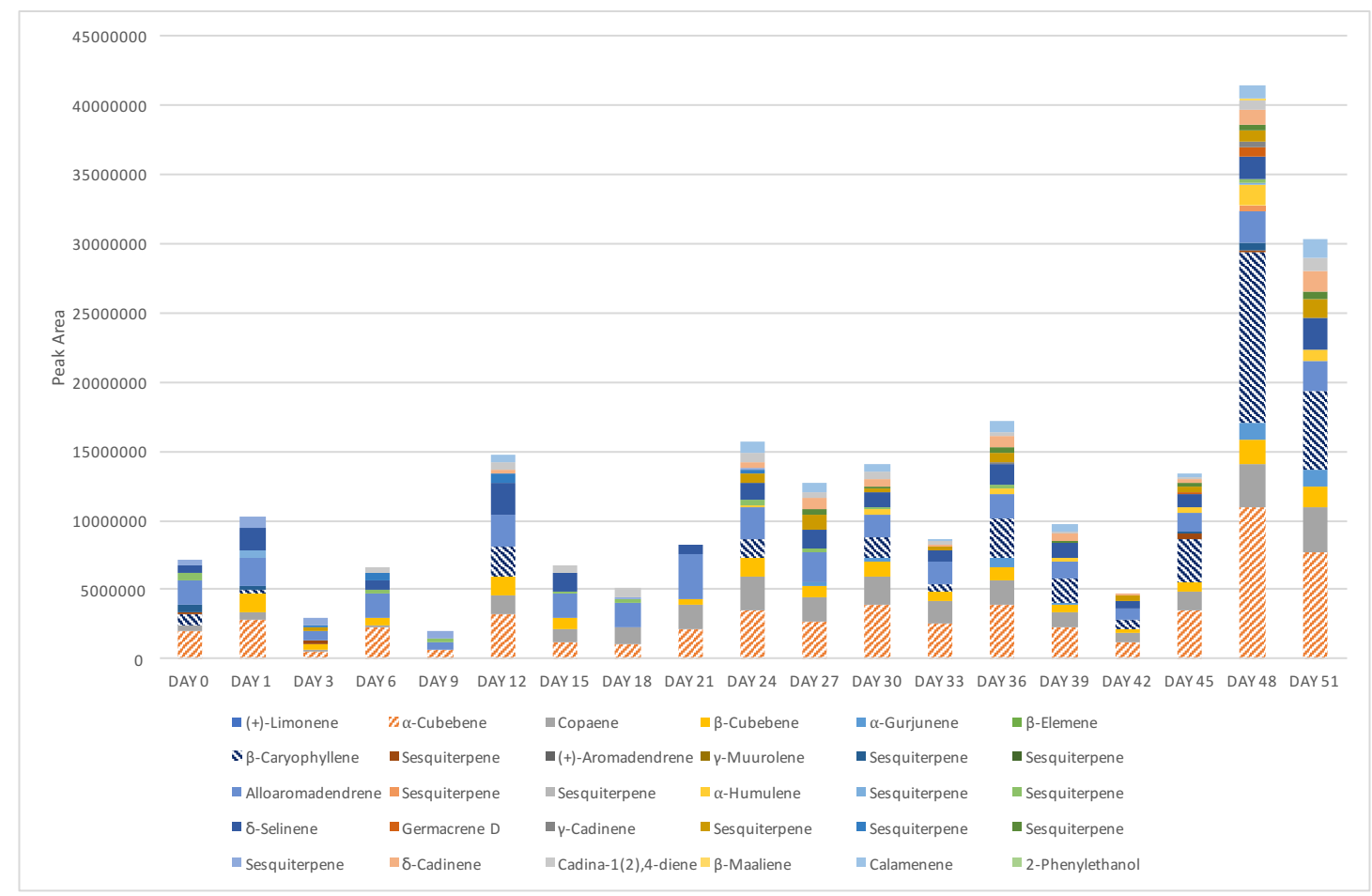

Figure 67: Odor profile of control avocado trees by sample day for Section 5.4.1.

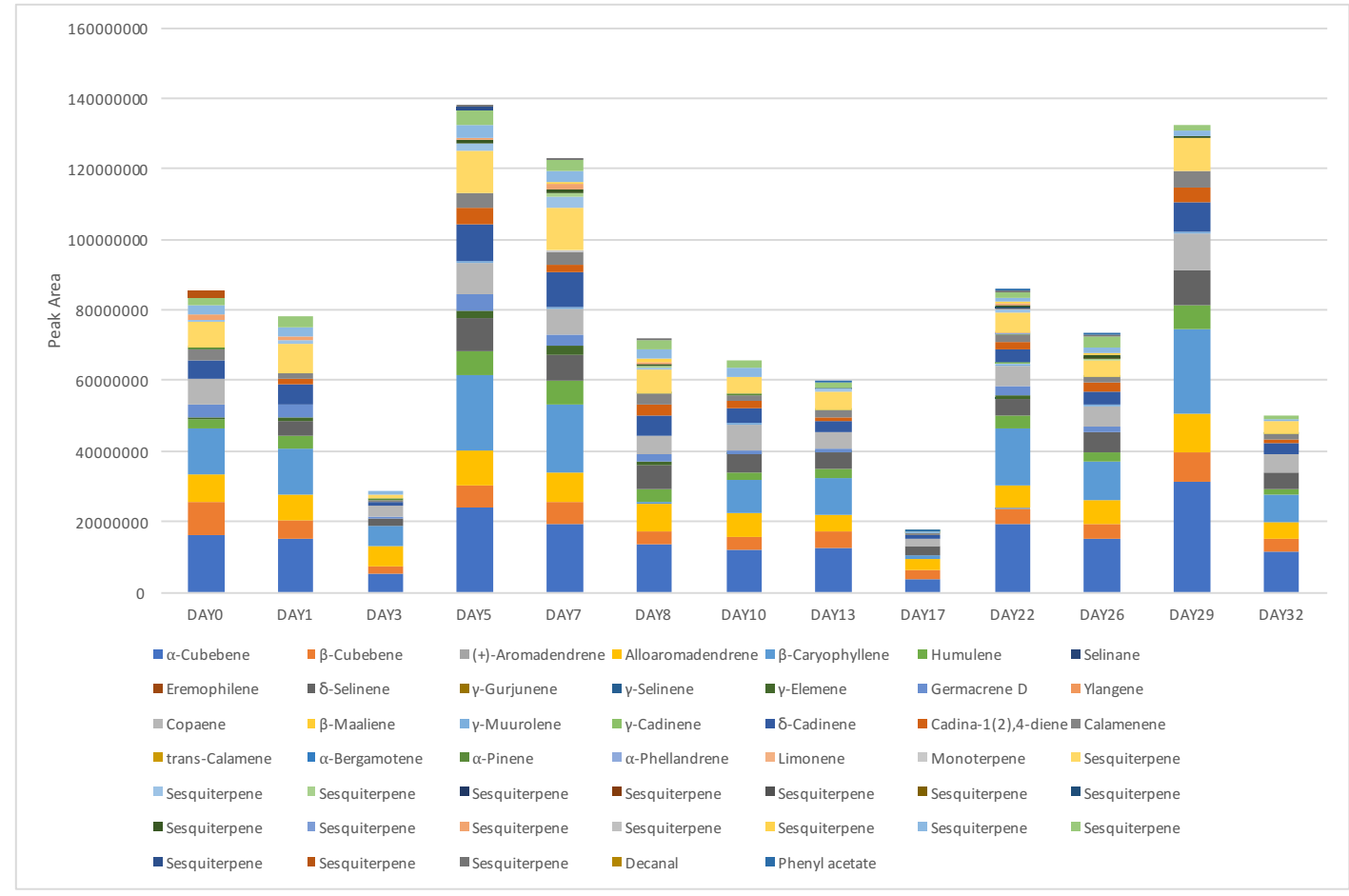

Figure 68: Odor profile of avocado trees inoculated with $R$. lauricola and then treated with propiconazole by sample day for Section 5.4.2. 


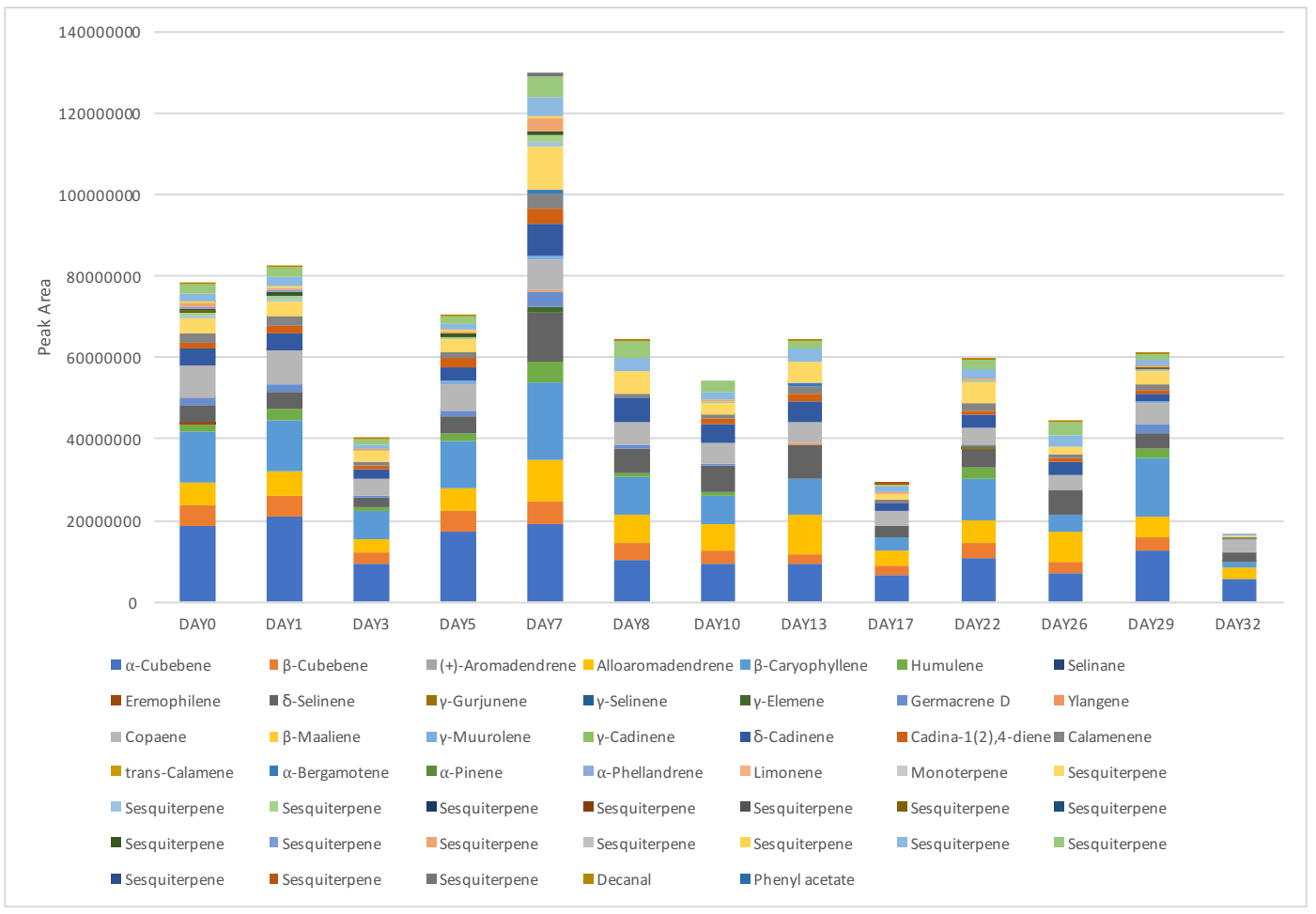

Figure 69: Odor profile of avocado trees inoculated with $\boldsymbol{R}$. lauricola and left untreated by sample day for Section 5.4.2.

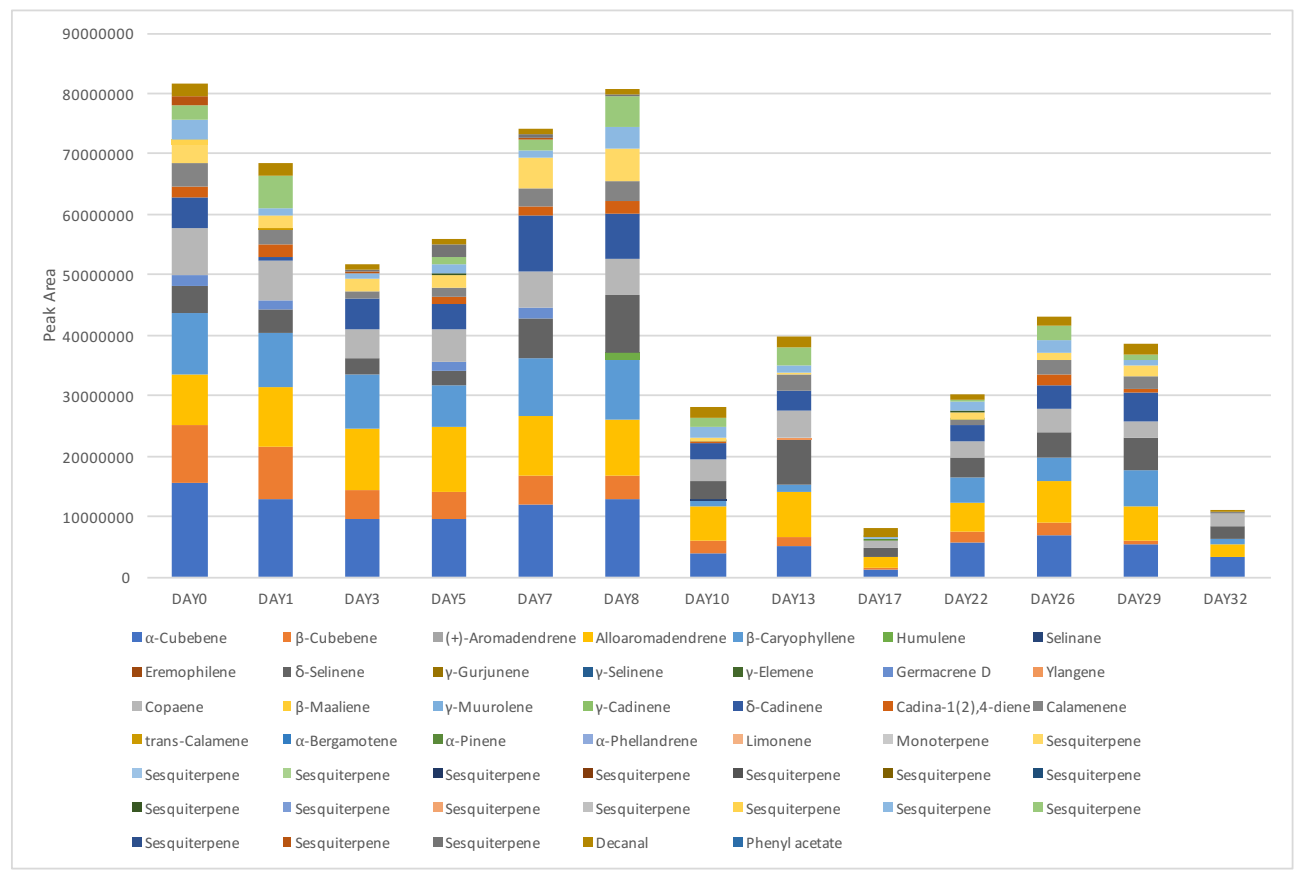

Figure 70: Odor profile of control avocado trees (not inoculated with $R$. lauricola but treated with propiconazole) by sample day for Section 5.4.2. 


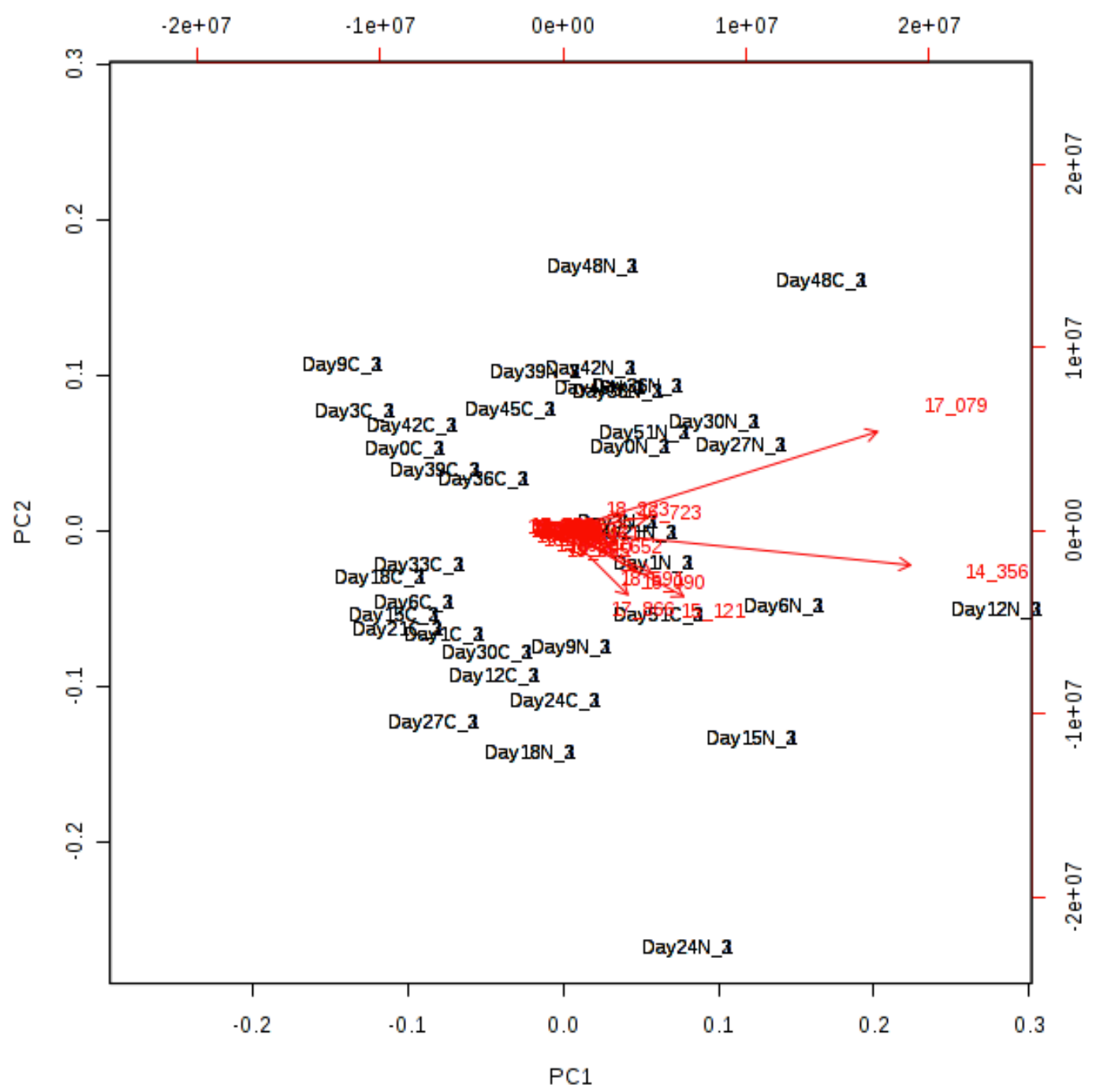

Figure 71: PCA biplot (created from non-normalized data) for greenhouse experiment presented in Section 5.4.1. 17_079 is $\beta$-caryophyllene, $14 \_356$ is $\alpha$-cubebene, and $15 \_121$ is copaene. 


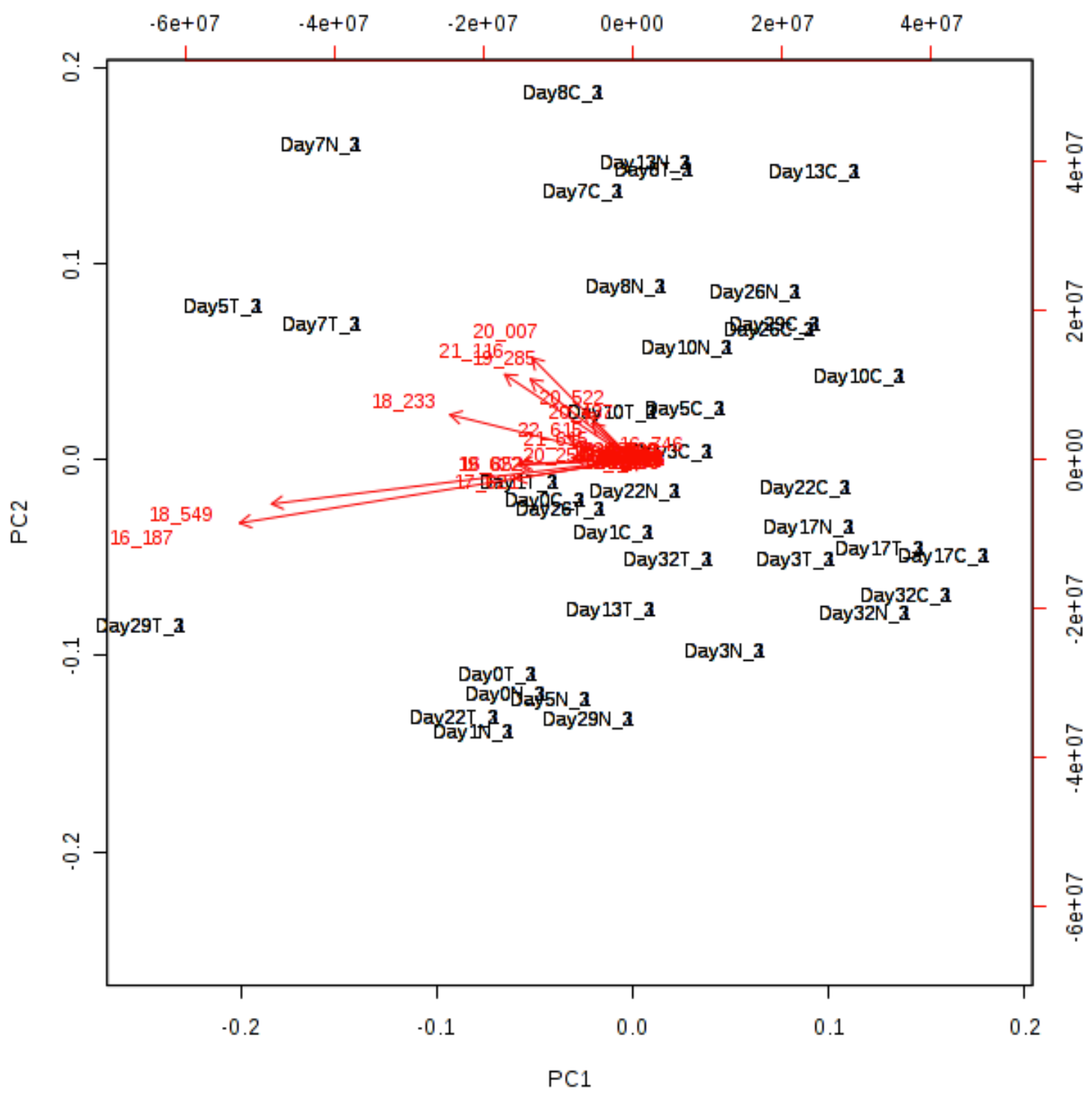

Figure 72: PCA biplot (created from non-normalized data) for greenhouse experiment presented in Section 5.4.2. 18_0549 is $\beta$-caryophyllene, $16 \_187$ is $\alpha$-cubebene, $18 \_233$ is an unidentified sesquiterpene, 20_007 is $\delta$ selinene, 21_116 is $\delta$-cadinene, and 19_285 is alloaromadendrene. 
VITA

\section{ALISON G SIMON}

2013-2016

2009-2013

2013-2017

2016

2014-2016

2015

2011-2014

2013

2012,2013
MS Forensic Science, Department of Chemistry and Biochemistry, Florida International University, Miami, FL Advisor: Kenneth G. Furton

BA Chemistry, Minor in Spanish

BS Criminal Justice

Texas Christian University, Fort Worth, TX

Advisor: Jason Clark-Miller

Canine Certifier, International Forensic Research Institute

Graduate Student Appreciation Week Scholarly Forum Winner, Florida International University

FBI Honors Internship Program

Evidence Response Team, Miami, FL

\$10,000 scholarship awarded by J. Edgar Hoover Foundation and Society of Former Special Agents of the FBI

FBI Volunteer Internship Program

Applicant Unit, Miami, FL Health Care Fraud and Public Corruption Squads, Dallas, TX Forensic Canine Program, FBI Laboratory, Quantico, VA

John V. Roach Honors College, Departmental Honors in Criminal Justice, Texas Christian University

Criminal Justice Departmental Field Research Award, Texas Christian University 


\section{PUBLICATIONS AND SELECTED PRESENTATIONS}

Simon, A.; Mills, D.; Furton, K. Chemotyping the temporal volatile organic compounds (VOCs) of an invasive fungus to the United States, Raffaelea lauricola. J Chrom A 2017, $1487,72-76$.

Simon, A.; Mills, D.; Furton, K. Chemical and canine analysis as complimentary techniques for the identification of active odors of the invasive fungus, Raffaelea lauricola. Talanta 2017, 168, 320-328.

Simon, A.; DeGreeff, L.; Peranich, K.; Furton, K. Evaluation of generalization discrimination balance for detection canines, International Defence \& Security Canine Conference, July 2017, Cirencester, UK (oral).

Simon, A.; Mills, D.; Furton, G. Chemical and canine analysis as complimentary techniques for the identification of active odors in an invasive agricultural pest in the USA, International Working Dog Conference, April 2017, Banff, Canada (oral).

Simon, A.; Furton, K. Greenhouse evaluations of volatile plant defense against an invasive agricultural and environmental biothreat agent, Raffaelea lauricola, Pittcon, March 2017, Chicago, IL (poster).

Simon, A.; Furton, K. Characterization of controlled odor mimic permeation systems (COMPS) containing live training aids for utilization by detection canines, American Academy of Forensic Science $69^{\text {th }}$ Annual Meeting, February 2017, New Orleans, LA (oral).

Simon, A.; Mills, D.; Furton, K. Chemical and canine analysis as complimentary techniques for the identification of active odors in an invasive species in the USA, $18^{\text {th }}$ International Symposium on Advances in Extraction Technologies \& $22^{\text {nd }}$ International Symposium on Separation Sciences, July 2-6, 2016, Torun, Poland (oral).

Simon, A.; Mills, D.; Furton, K. Volatile organic compounds (VOCs) of an invasive fungus to the United States, Raffaelea lauricola, and possible effects on commercial avocado groves, $18^{\text {th }}$ International Symposium on Advances in Extraction Technologies $\& 22^{\text {nd }}$ International Symposium on Separation Sciences, July 2-6, 2016, Torun, Poland (poster).

Simon, A.; Mendel, J.; Mills, D.; Furton, K. An assessment of the VOCs of an agricultural biothreat agent, Raffaelea lauricola, and training aids for canine detection, Graduate Student Appreciation Week, April 2015, Miami, FL. Scholarly Forum Winner (poster). 